DIPLOMARBEIT

\title{
Einfluss von Interpretationshilfen auf die Benützung von komplexen interaktiven thematischen Karten
}

ausgeführt zum Zwecke der Erlangung des akademischen Grades einer

Diplom-Ingenieurin unter der Leitung von

Prof. Dr. Georg Gartner und

Dipl.-Ing. (FH) Manuela Schmidt

E120 Department für Geodäsie und Geoinformation

eingereicht an der Technischen Universität Wien

Fakultät für Architektur und Raumplanung

von

Manuela Stögerer

Matr. Nr.: 0726311

Wien, am 1.4.2015

Unterschrift (Studentin) 


\section{KURZFASSUNG}

Der technische Fortschritt und die Verbreitung des Internets ermöglichen es räumliche Informationen durch hoch dynamische interaktive digitale Karten an eine Vielzahl von Nutzenden zu kommunizieren. Jedoch stoßen die beinahe unendlichen technischen Möglichkeiten auf die begrenzten visuellen und kognitiven Verarbeitungsfähigkeiten des Menschen. Verschiedene Konzepte zu Nutzerführung und Hilfestellung werden eingesetzt, um die Nutzenden bei ihrer Benützung zu unterstützen.

Diese Arbeit befasst sich mit der Frage ob Interpretationshilfen eine geeignete Methode sind um Nutzende bei der Benützung von komplexen interaktiven thematischen Karten zu unterstützen. Relevant ist dabei zu untersuchen, welche Inhalte durch die Implementierung der Interpretationshilfe (richtig) interpretiert werden und wie sich ihr Einsatz auf das Explorationsverhalten der Nutzenden auswirkt. Gestützt wird die Untersuchung auf kognitive Aspekte sowie auf Konzepte zu Nutzerführungen und Hilfestellungen in unterschiedlichen Softwareprodukten. Die Beantwortung der Fragestellung erfolgt besonders unter der Berücksichtigung perzeptiver Regeln, dem Aufmerksamkeitsverhalten der Nutzenden sowie den Erfahrungen zu bereits umgesetzten Nutzerführungskonzepten.

Durch einen summativen Usability-Test wird der Einfluss der Interpretationshilfe anhand einer komplexen interaktiven thematischen Karte getestet. Um dabei auf die Vertrautheit der Nutzenden im Kartenlesen Rücksicht zu nehmen, werden zwei Testgruppen gebildet. Quantitative Daten, die mittels Leistungstests erhoben werden, als auch qualitative Daten, die durch Fragebögen gewonnenen werden, ermöglichen es, die Forschungsfrage und ihre Hypothesen zu beantworten.

Der Vergleich zwischen interpretierten und wiedergegebenen Inhalten in Karten mit und ohne implementierter Interpretationshilfe führt zum Ergebnis, dass die Interpretationshilfe als eine hilfreiche Methode zur Benützung von komplexen interaktiven thematischen Karten zu sehen ist. Es werden überwiegend jene Informationen von den Nutzenden aufgenommen, die von der Interpretationshilfe aufgegriffen und beschrieben werden. Vor allem bei im Kartenlesen ungeübten Nutzenden, sowie bei Karten zu unbekannten Orten, ist der leitende Einfluss der Interpretationshilfe zu erkennen. 


\section{ABSTRACT}

Technical advances and the availability of the internet make it possible to easily provide highly dynamic interactive digital maps to a wide range of users. These almost endless technological possibilities are confined by the visual and cognitive processing capabilities of the map readers. Assistance and help systems are therefore needed to support users in their tasks.

This thesis examines the question whether Assistants for Interpretation are useful tools to facilitate the usage of Complex Interactive Thematic Maps. It researches the influence of this application in the users' interpretation and exploration behaviour of maps. The evaluation of the Assistant for Interpretation is based on cognitive aspects and concepts for user guidance and help systems of various software products. In order to answer the research question, perception and observation of attentional behaviour of users as well as experiences from extant help systems or user guides were used.

Furthermore, the author used a summative usability test to investigate the influence of the Assistant for Interpretation in a Complex Interactive Thematic Map. With regard to previous experience in map usage, users are divided into two subgroups. Quantitative data gained through performance tests as well as qualitative data gained through questionnaires are used to answer the research question.

The findings indicate that the Assistant for Interpretation is a suitable tool to facilitate the usage of Complex Interactive Maps. The contents of the map that got addressed by the Assistant for Interpretation are more likely to be repeated correctly. This influence is especially notable in novice map users as well as in maps about unknown areas. 


\section{DANKSAGUNG}

An dieser Stelle möchte ich mich bei all denjenigen bedanken, die mich im Zuge meiner Diplomarbeit unterstützt haben. Ein besonderen Dank gilt Dipl.-Ing.(FH) Manuela Schmidt und Univ. Professor Georg Gartner, die mir beim Verfassen dieser Arbeit stets unterstützend zur Seite standen.

Ebenfalls großer Dank geht an meine Testpersonen, die sich bereitwillig für die Teilnahme am Usability-Test zur Verfügung gestellt haben. Dank der Motivation jedes Einzelnen konnte ein interessanter und aufschlussreicher Test durchgeführt werden.

Mein Dank gilt auch meiner Mutter sowie meiner Schwester für das Korrekturlesen der Arbeit, meinen Freunden für motivierenden Worte und generell meinen Eltern für die Unterstützung während meines Studiums.

Die Zitierung der Literaturquellen erfolgt nach der Harvard-Zitierweise. Englischsprachige Begriffe werden in kursiv dargestellt. Weiters wurde beim Verfassen dieser Arbeit auf eine genderneutrale Formulierung geachtet. 


\section{INHALTSVERZEICHNIS}

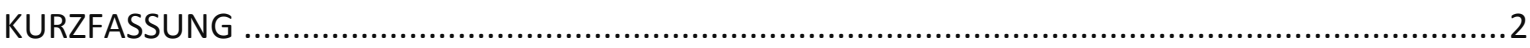

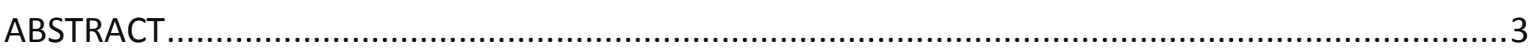

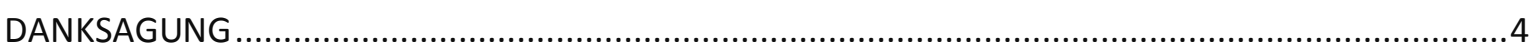

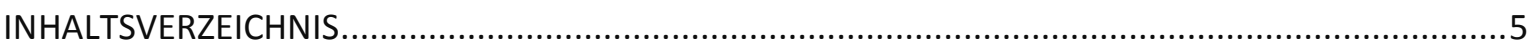

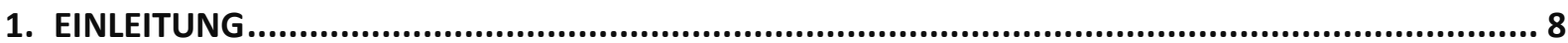

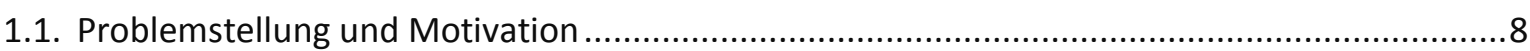

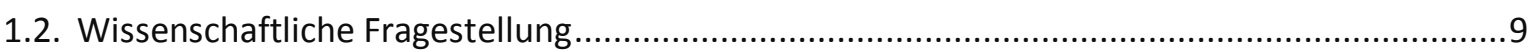

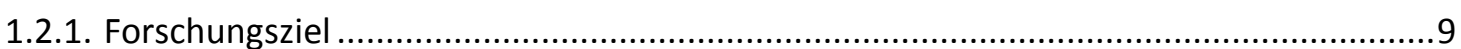

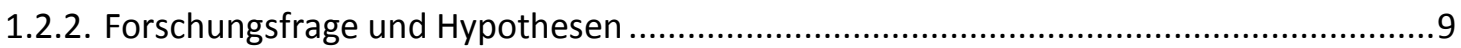

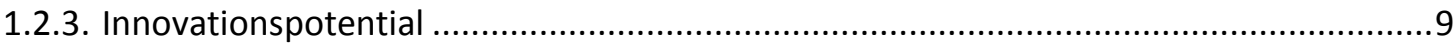

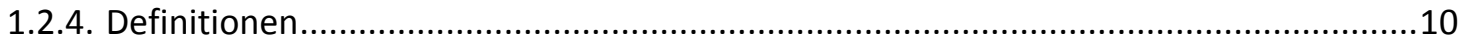

1.2.4.1. Benützung, Verständnis, Interpretation .................................................... 10

1.2.4.2. Komplexe interaktive thematische Karten (KIT-Karten) .................................10

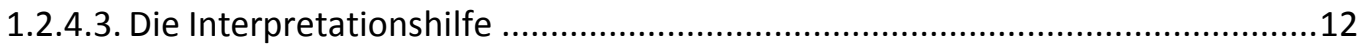

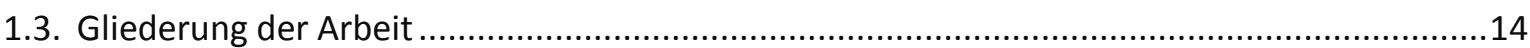

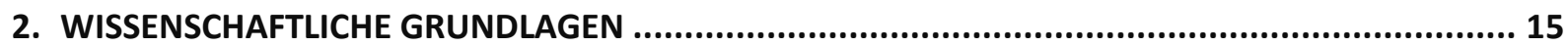

2.1. Thematische Kartographie und KIT-Karten ......................................................................... 15

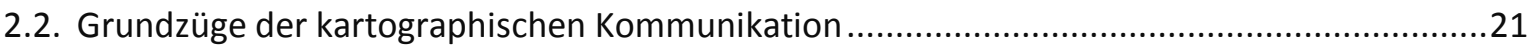

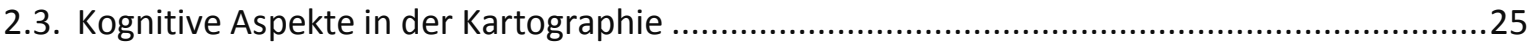

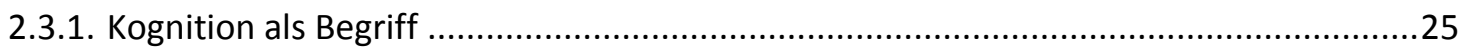

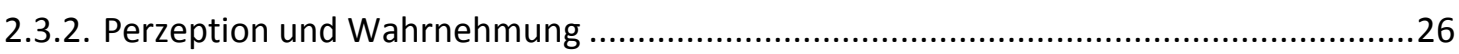

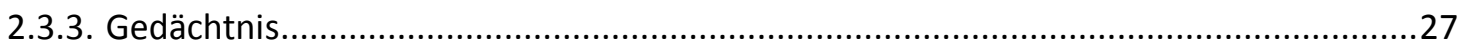

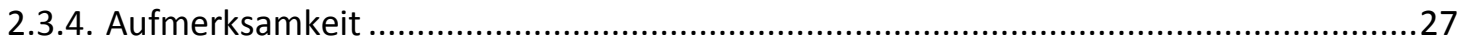

2.3.4.1. Selektive vs. geteilte Aufmerksamkeit ..........................................................28

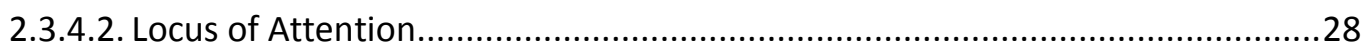

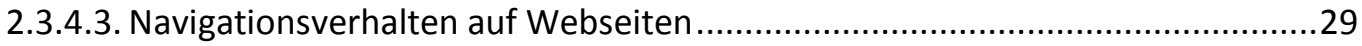

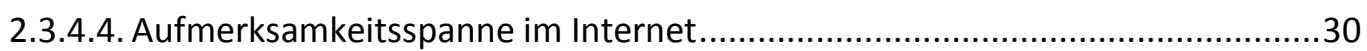

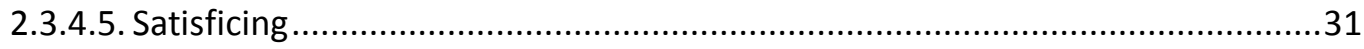

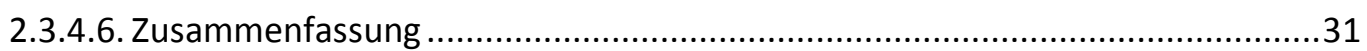

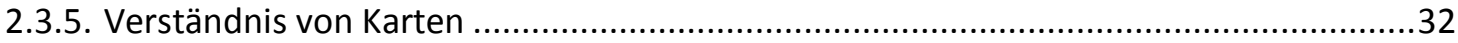

2.3.6. Zusammenfassung und Hypothesen zu kognitiven Aspekten .......................................33

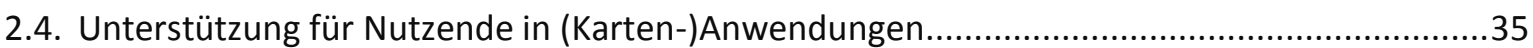

2.4.1. Benutzersteuerung und -kontrolle in interaktiven Kartenprodukten ............................36

2.4.1.1. Metaphern zur Kommunikation geographischer Information ..........................36

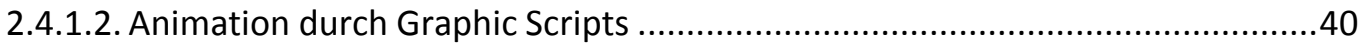

2.4.1.3. Engineered Serendipity in Kartenprodukten................................................... 40 
2.4.1.4. Erzähltechniken in Narrative Visualizations .................................................. 41

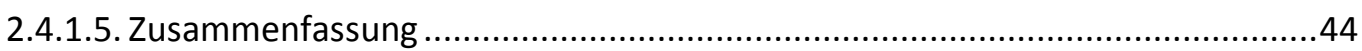

2.4.1.6. Interpretationshilfe und Hypothese zu Nutzerführungen in Karten ..................45

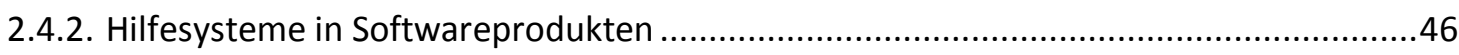

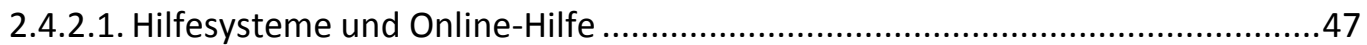

2.4.2.2. Implementierungsmöglichkeiten von Online-Hilfen ......................................48

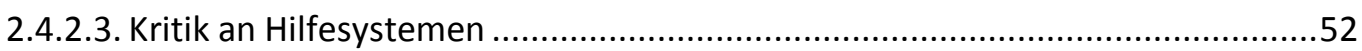

2.4.2.4. Zusammenfassung ....................................................................................... 53

2.4.2.5. Interpretationshilfe und Hypothesen zur Unterstützung von Nutzenden .........54

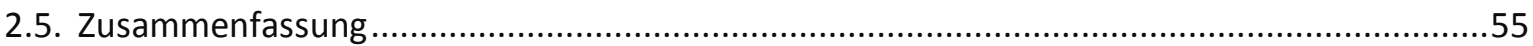

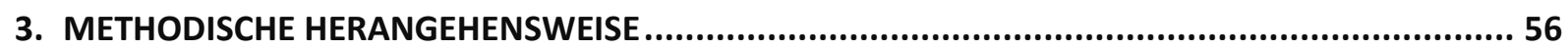

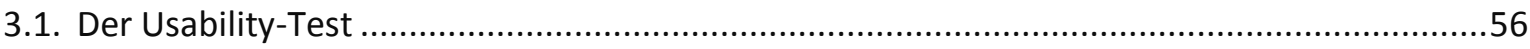

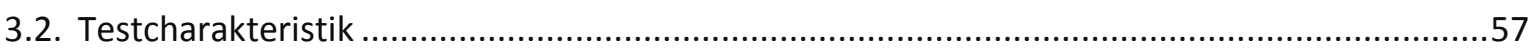

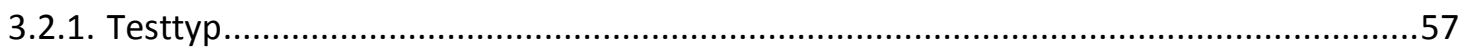

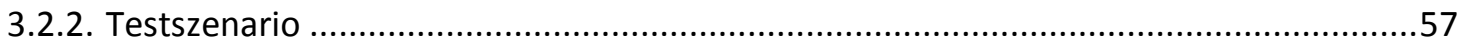

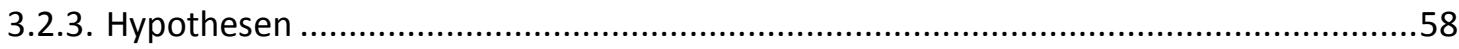

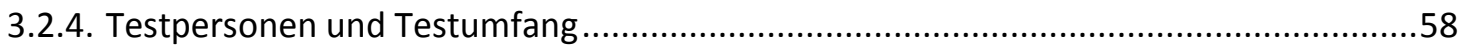

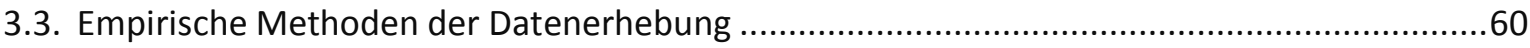

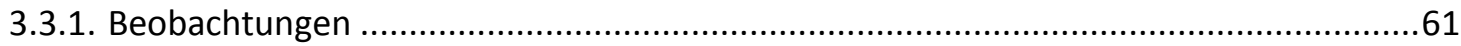

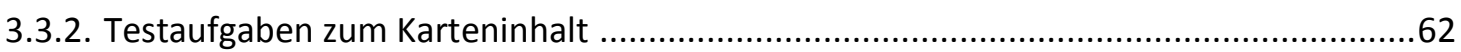

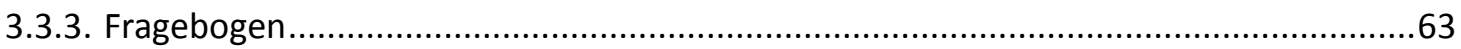

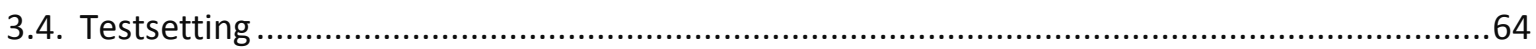

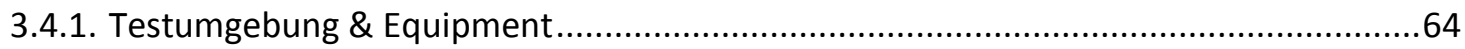

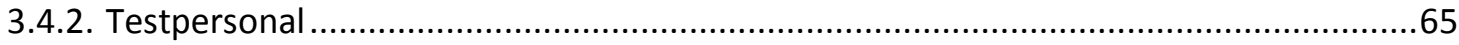

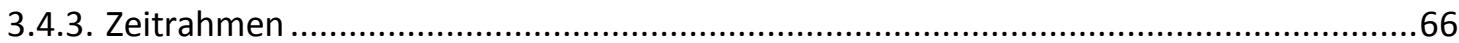

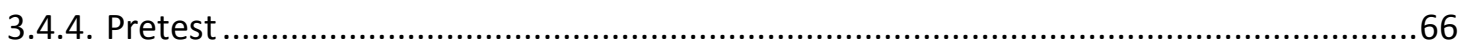

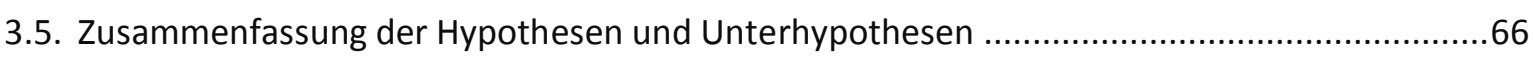

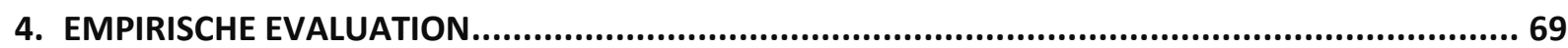

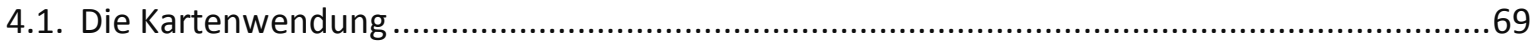

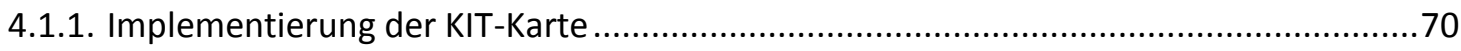

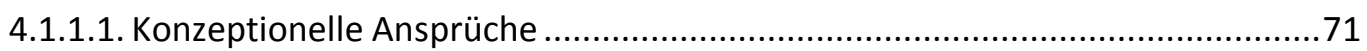

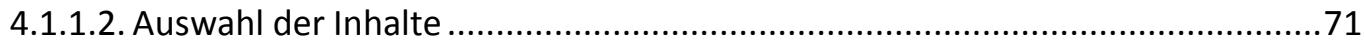

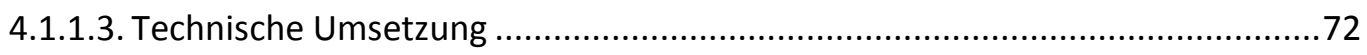

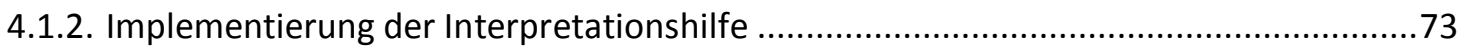

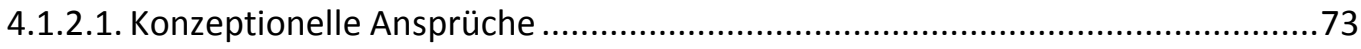

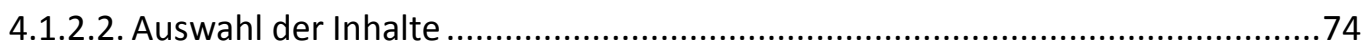

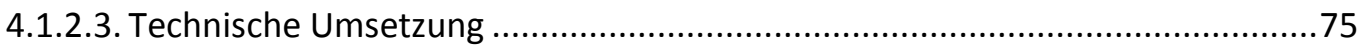




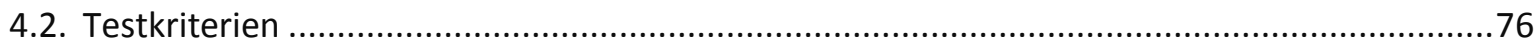

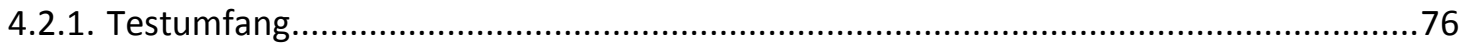

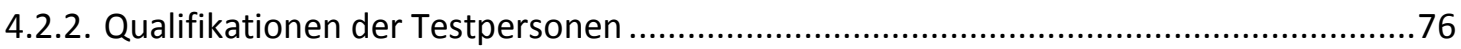

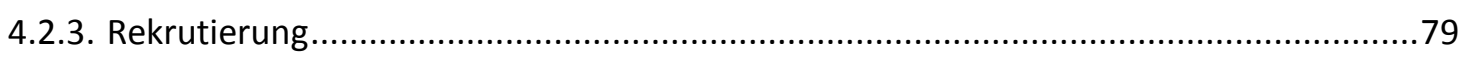

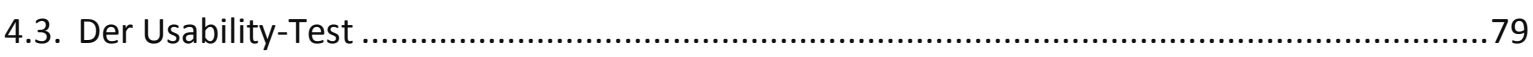

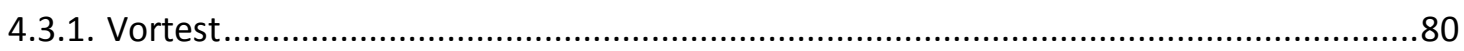

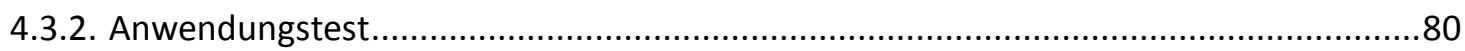

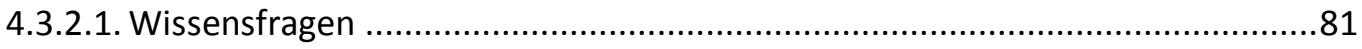

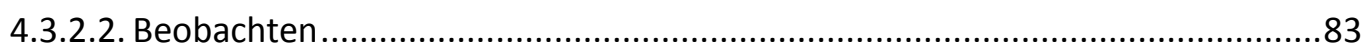

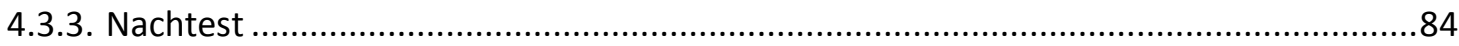

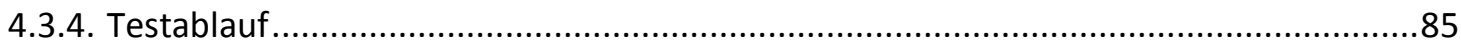

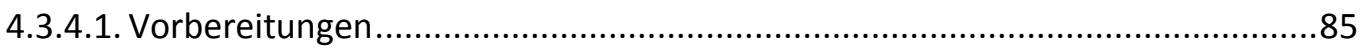

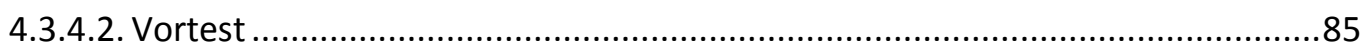

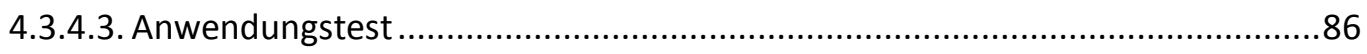

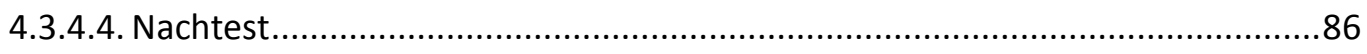

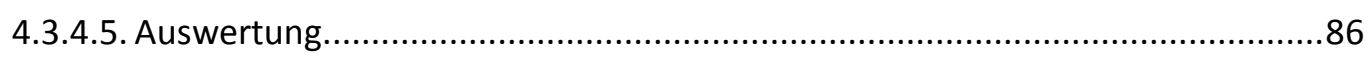

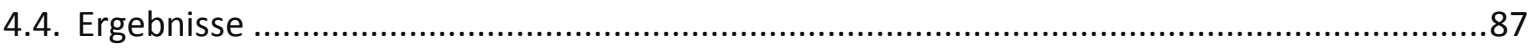

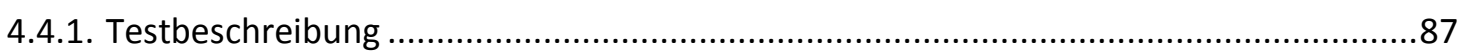

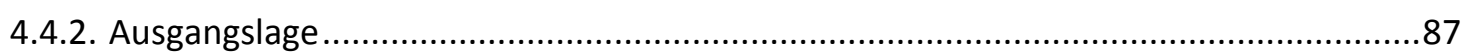

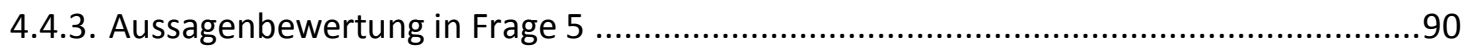

4.4.4. Evaluation der Hypothesen ......................................................................................91

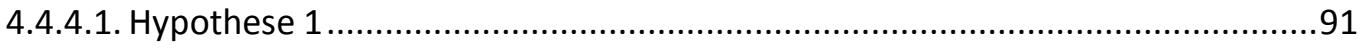

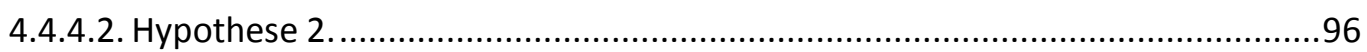

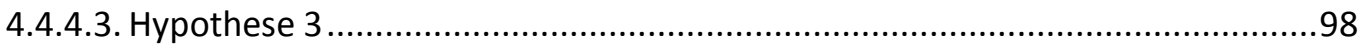

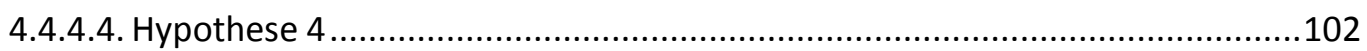

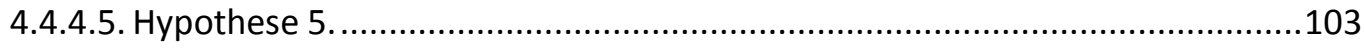

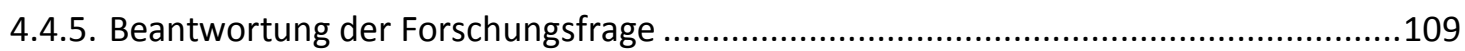

4.4.6. Einschränkungen und Signifikanz der Testergebnisse ...............................................109

5. RESÜMEE

5.1. Zusammenfassung über die "Interpretationshilfe"...........................................................114

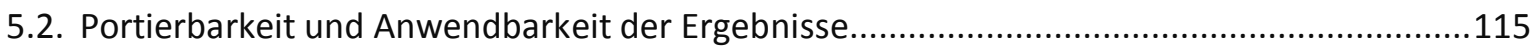

5.3. Kritik am Einsatz von Interpretationshilfen .................................................................... 115

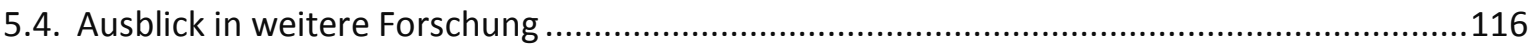

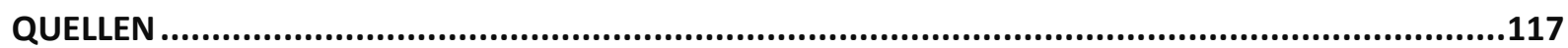

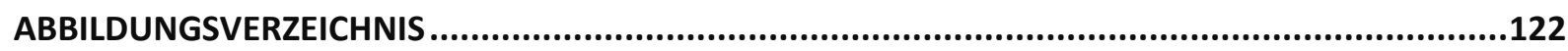

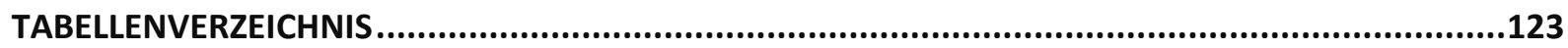

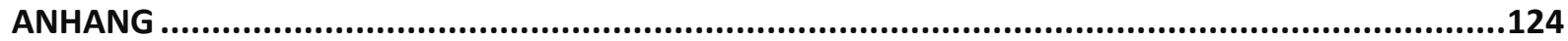




\section{EINLEITUNG}

Aufgrund des stetig voranschreitenden technischen Fortschritts der letzten Jahrzehnte vollzog sich die Kartographie einer enormen Entwicklung (Ooms 2012). Vor allem die Allgegenwärtigkeit des Internets hat die Art und Weise verändert, wie Karten heute verwendet werden. Hoch dynamische interaktive digitale Karten, die über das Internet präsentiert werden, haben immer mehr an Bedeutung gegenüber statisch analogen Formaten gewonnen (Ooms 2012). Durch das Internet sind Karten und Kartographie für die Allgemeinheit viel leichter zugänglich geworden. Diese Umstände fordern, sich mit den neuen Herausforderungen auseinander zu setzen.

In diesem Kapitel werden der Ausgangspunkt und die Relevanz dieser Arbeit dargelegt. Anschließend wird die Forschungsfrage und ihre Hypothesen vorgestellt und das methodische Vorgehen dieser wissenschaftlichen Arbeit beschrieben.

\subsection{PROBLEMSTELLUNG UND MOTIVATION}

Schon seit mehr als 35000 Jahren sind bildliche Darstellungen für die Kommunikation von Ideen essentiell (Smiciklas 2012). Heute stehen durch technische Entwicklungen von Hardware- und Softwareprodukten sowie weitgehender Verfügbarkeit von Daten der kartographischen Gestaltung von Karten viele Möglichkeiten offen (Harrower 2007). Komplexe interaktive thematische Karten sollen dem Anspruch, Informationen schnell zu kommunizieren, gerecht werden.

Die Freiheiten, die durch aktuelle Entwicklungen seitens technischer Umsetzungsmöglichkeiten entstehen, führen auf der Nutzerseite allerdings zu Restriktionen. Durch beschränkte visuelle und kognitive Verarbeitungsfähigkeiten (processing capabilities) des Menschen können Kartennutzende bei komplexen Kartendarstellungen schnell überfordert sein (Harrower 2007). Online präsentierte Karten sind zusätzlich mit der verkürzten Aufmerksamkeitsspanne der Betrachter im Web konfrontiert (Weinreich et al. 2008).

Um die Nutzenden unter diesen Umständen darin zu unterstützen, komplexe interaktive Karten zu verstehen und richtig zu interpretieren, soll in der vorliegenden Arbeit der Einsatz von Interpretationshilfen untersucht werden. Unter Berücksichtigung der Kartennutzenden und ihren Fähigkeiten wird in Kombination mit Erfahrungen aus bereits implementierter Unterstützung für Nutzende in (Karten-)Produkten, der Einfluss der Interpretationshilfe auf die Benützung der Karte evaluiert. Durch die empirische Methode einer Usability-Test Durchführung werden die Auswirkungen der Interpretationshilfe anhand eines Beispiels zu einer komplexen interaktiven thematischen Karte untersucht. 


\subsection{WISSENSCHAFTLICHE FRAGESTELLUNG}

\subsubsection{FORSCHUNGSZIEL}

Ziel der Arbeit ist es, die Einflüsse einer Interpretationshilfe auf die Benützung von komplexen interaktiven thematischen Karten zu identifizieren. Durch die Evaluation können Aussagen zu Vor- und Nachteilen beim Einsatz einer Interpretationshilfe in komplexen interaktiven thematischen Karten gemacht werden.

Diese Arbeit beschäftigt sich nicht mit der Bewertung der allgemeinen Benutzerfreundlichkeit von komplexen interaktiven thematischen Karten. Auch die verschiedenen Möglichkeiten zur Implementierung der Interpretationshilfe (neben der Karte, über der Karte, zwingend, freiwillig, ...) stehen nicht im Fokus der Arbeit.

\subsubsection{FORSCHUNGSFRAGE UND HYPOTHESEN}

Die Forschungsfrage, die in dieser Arbeit beantwortet werden soll, lautet:

Ist eine Interpretationshilfe eine geeignete Methode um die Benützung von komplexen interaktiven thematischen Karten zu unterstützen?

Die Forschungsfrage wird durch das Testen von fünf Hypothesen beantwortet:

HYPOTHESE 1 Die Verwendung einer Interpretationshilfe führt zur Interpretation von bestimmten Inhalten.

HYPOTHESE 2 Durch die Verwendung einer Interpretationshilfe werden die dargestellten Inhalte häufiger korrekt interpretiert.

HYPOTHESE 3 Die Verwendung einer Interpretationshilfe führt zu einem verminderten Explorieren in der Karte.

HYPOTHESE 5 Die Interpretationshilfe wird von den Testpersonen als hilfreich und unterstützend wahrgenommen.

HYPOTHESE 4 Die Testpersonen fühlen sich von der Interpretationshilfe bevormundet.

\subsubsection{INNOVATIONSPOTENTIAL}

Diese Arbeit betrachtet die Interpretationshilfe in komplexen interaktiven thematischen Karten hinsichtlich der Frage nach ihren Auswirkungen auf die Benützung der Karte: Sind Interpretationshilfen hilfreich um sich in komplexer Interaktivität zurecht zu finden? Der Vergleich zwischen interpretier- 
ten und wiedergegebenen Inhalten in Karten mit und ohne Interpretationshilfe soll Aufschluss über ihre Auswirkungen geben.

Wissen über den Einfluss einer Nutzerführung durch eine Interpretationshilfe auf das Erfassen von Karteninhalten ist sowohl für den Nutzenden als auch für Kartenersteller von Bedeutung. Die gewonnenen Kenntnisse können sowohl gezielt zu Erstellung von komplexem interaktiven thematischen Karten verwendet werden, als auch den Nutzenden einen bewussteren Umgang mit den dargestellten Informationen ermöglichen.

\subsubsection{DEFINITIONEN}

Um die Forschungsfrage zu formulieren und sie thematisch einzuordnen, werden bestimmte Begrifflichkeiten verwendet. Im Folgenden wird festgelegt, wie die diese im Rahmen der Arbeit verwendet werden.

\subsubsection{Benützung, Verständnis, Interpretation}

'Benützung', 'Verständnis' und 'Interpretation' sind zentrale Begriffe bei der Untersuchung der Interpretationshilfe. Bei diesen Wörtern handelt es sich nicht um spezielle Fachausdrücke, sondern um im Alltag gebräuchliche Wörter. Im thematischen Hintergrund dieser Arbeiten werden diese Begriffe in einem bestimmten Kontext verwendet. Um festzulegen, was diese Begriffe im Zusammenhang dieser Arbeit umfassen, werden sie an dieser Stelle kurz erläutert.

\section{Benützung}

Unter 'Benützung' versteht man den allgemeinen Gebrauch von Dingen, wie bspw. auch Karten. Die Benützung von Karten umfasst die Art und Weise, wie die Kartennutzenden die Karte verwenden. Sie kann sich dabei bspw. hinsichtlich Explorationsverhalten oder Möglichkeiten zur Navigation und Orientierung unterscheiden. Wie die Kartennutzenden im Zusammenhang dieser Arbeit komplexe thematische Karten benützen, wird durch den Usability-Test festgestellt.

\section{Interpretation}

'Interpretation' meint hinsichtlich der Verwendung von Karten das Wahrnehmen, Lesen und Sinn ableiten aus den in der Karte dargestellten Elementen. Interpretation steht dabei im Zusammenhang mit der Dekodierung von kartographischen Symbolen und Zeichen und dem herstellen von Zusammenhängen der dargestellten Aussageebenen.

\section{Verständnis}

'Verständnis' bedeutet laut Duden Auffassung oder Deutung. Je nachdem wie die einzelnen Elemente der Karte interpretiert werden, ergibt sich ein Gesamtbild der Karte und wird übergreifend das als 'Verständnis' der Karte bezeichnet. 'Verständnis' beschreibt demnach jene Information, die die Nutzende durch die Benützung der Karte ableiten.

\subsubsection{Komplexe interaktive thematische Karten (KIT-Karten)}

Als KIT-Karte (Komplexe interaktive thematische Karte) wird im Folgenden ein Kartenprodukt bezeichnet, das einer besonderen Form der thematischen Karte entspricht. Der Begriff der KIT-Karte 
wurde eigens zum Zweck dieser Arbeit definiert, um bestimmte Eigenschaften eines Kartenprodukts vorauszusetzen.

Wie Kapitel 2.1 beschrieben wird, unterscheiden sich KIT-Karten von der klassischen Verwendung des Begriffs 'Komplexität' in der thematischen Kartographie, der sich lediglich auf die Eigenschaften einer einzelnen Kartendarstellung beschränkt. Bei KIT-Karten handelt es sich jedoch nicht um einzelne, unabhängige Karten, sondern um ein kartographisches Produkt, in dem verschiedene Karten und Aussageebenen miteinander kombiniert werden. Inhaltlich werden verschiedene thematische Merkmale im Bezug auf ihre räumlichen Ausprägungen abgebildet. Die Daten werden nicht nur durch klassische Kartenabbildungen visualisiert, sondern mit weiteren Darstellungsformen, wie beispielsweise Diagrammen, kombiniert. Daraus ergibt sich eine Komplexität für die KIT-Karte, die einerseits durch die Überlagerung mehrerer Aussageebenen als auch durch die Kombination verschiedener Visualisierungsmethoden (Karte oder Diagramm) entsteht. Um dieser Komplexität zu begegnen wird die KIT-Karte mit interaktiven Elementen ausgestattet. KIT-Karten verfügt weiters über eine ansprechende Ästhetik sowie einfache und unkomplizierte Bedienung. Dieses ist notwendig um einen ansprechenden Zugang zu den dargestellten Inhalten zu ermöglichen. Tabelle 1-1 gibt einen Überblick über die wesentlichen Eigenschaften der KIT-Karte.

\begin{tabular}{|c|c|}
\hline KARTE & Ansprechende Visualisierung räumlicher Daten \\
\hline KOMPLEX & $\begin{array}{l}\text { - Mehrschichtige thematische Aussageebenen } \\
\text { - Kombination klassischer Kartendarstellung mit } \\
\text { weiteren Darstellungsformen (bspw. Diagramme) }\end{array}$ \\
\hline INTERAKTIV & $\begin{array}{l}\text { Elemente zur Navigation, Filtern, Suchen, } \\
\text { Zoomen, Anklicken oder Mouse-Over-Funktionen }\end{array}$ \\
\hline THEMATISCH & $\begin{array}{l}\text { Vorrangig keine topographischen Inhalte sondern } \\
\text { Darstellung eines Sachverhalts }\end{array}$ \\
\hline
\end{tabular}

Tabelle 1-1

Wesentliche Eigenschaften von KITKarten

(eigene Darstellung)

Anwendung finden KIT-Karten in verschiedenen Zusammenhängen. Vorwiegend ist sie im Internet auf Webseiten von großen Nachrichtenorganisationen, wie beispielsweise New York Times oder DIE ZEIT, sowie auf Blogs zu bestimmten Themen zu finden. Abbildung 1-1 zeigt ein Beispiel zur Anwendung einer KIT-Karte. 


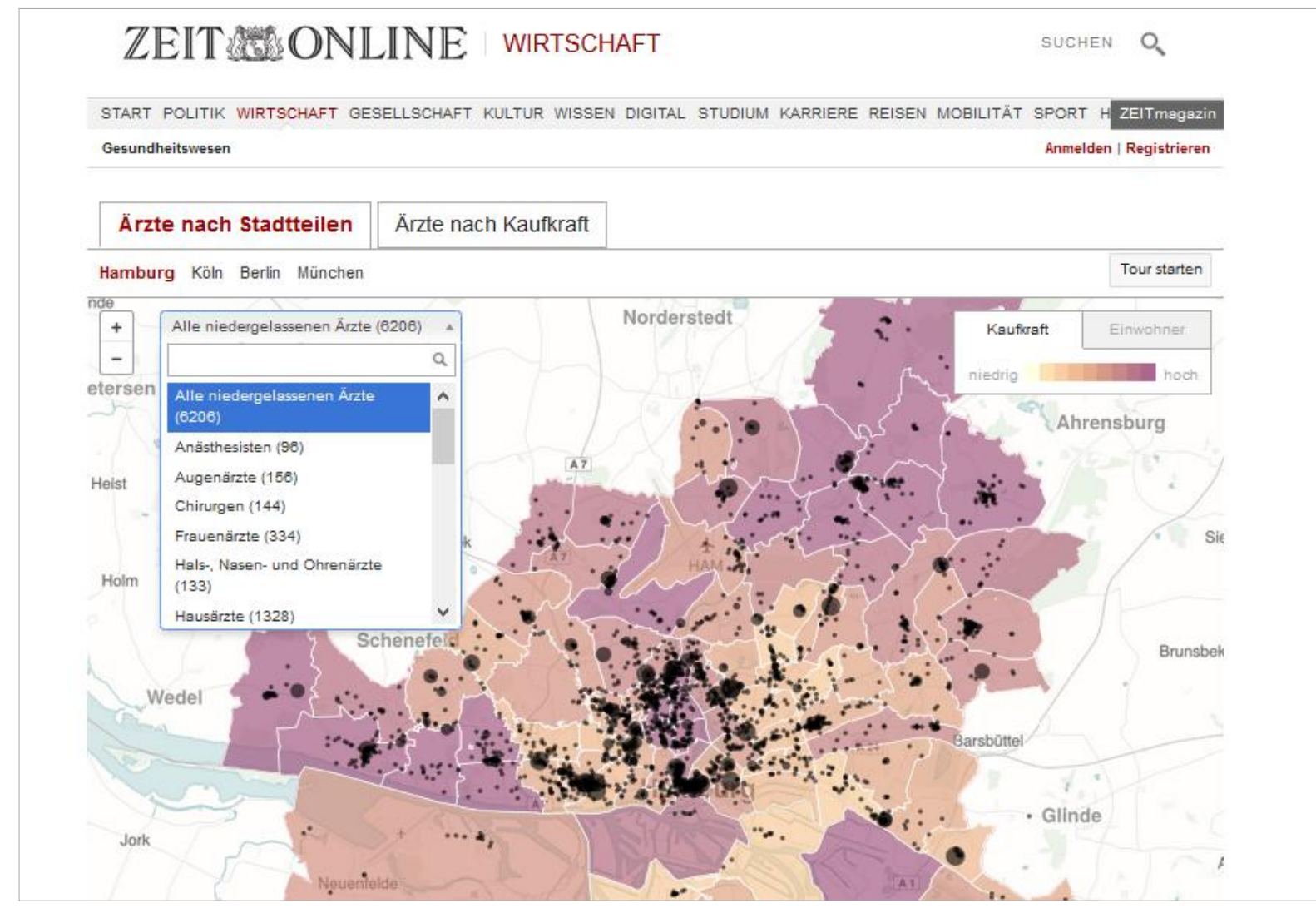

Abbildung 1-1 Beispiel einer KIT-Karte: Ausschnitt aus ZEIT ONLINE Karte "Geld steuert Ärzte" (ZEIT ONLINE 2014)

\subsubsection{Die Interpretationshilfe}

Wie in Kapitel 2.4 gezeigt wird, gibt es eine Vielzahl an Möglichkeiten, wie Nutzende bei der Benützung von (Karten-)Anwendungen unterstützt werden. Der Interpretationshilfe werden im Zuge dieser Arbeit spezielle Attribute zugeschrieben, die nicht vollständig einem in der Literatur beschriebenen Konzept zuzuordnen ist.

Ziel der Interpretationshilfe ist es, das Verständnis des dargestellten Inhalts zu fördern. Durch sie soll sichergestellt werden, dass Zusammenhänge, die die Kartenersteller kommunizieren wollen, auch von den Nutzenden wahrgenommen und verstanden werden können. Sie soll das Verständnis von räumlichen Daten fördern, indem durch klare Aussagen auf relevante thematische Zusammenhänge hingewiesen wird, die für das Erfassen der Kartenaussage wichtig sind.

Einsatz findet die Interpretationshilfe in KIT-Karten, die durch einen hohen Grad an Interaktivität und Komplexität Unterstützungen für Nutzende fordern. Die Kartenersteller erhalten durch diese Option die Möglichkeit, Überlegungen und Beobachtungen zu unterschiedlichen Inhalten der Karte in die Darstellungen einzubetten. Den Nutzenden werden Informationen zu gewissen Ausprägungen und Zusammenhängen zusammengestellt und präsentiert, die sie möglicherweise selbstständig nicht wahrgenommen und identifiziert hätten. Die Interpretationshilfe bezieht sich nur auf tatsächlich abgebildete Merkmale in der Karte, zusätzliches Hintergrundwissen wird demnach durch sie nicht zur Verfügung gestellt. 
Umgesetzt wird die Interpretationshilfe in dieser Arbeit als textlicher Kommentar, der zur Erläuterung des Inhalts der Darstellung in der KIT-Karte implementiert wird. Die Nutzenden werden entlang eines 'Pfades' durch die Inhalte geleitet, die für sie zusammengestellt werden. Die inhaltlichen Beschreibungen unterstützen bei der Interpretation der kartographischen Kodierung und helfen aus dargestellten Punkten, Linien und deren Verteilung Sinn abzuleiten. Die Unterstützung erfolgt in rein schriftlicher Form; graphische Hilfen, wie z.B. Pfeile, werden nicht verwendet. Weiters stellt die Interpretationshilfe keine technische Verwendungshilfe dar, die den Nutzenden die Anwendung erklärt, noch gibt sie zusätzliches Hintergrundwissen zu der dargestellten Thematik bekannt. Sie stellt auch keine direkten Aufforderungen an die Nutzenden, sich mit der Karte auseinanderzusetzen.

In Tabelle 1-2 wird ein Überblick über die Aufgaben und Funktionen der Interpretationshilfe gegeben. In Kapitel 4.1.2 wird genauer auf die Charakteristik der Interpretationshilfe und Art der Implementierung eingegangen, die im Zuge dieser Arbeit untersucht wird.

\begin{tabular}{|c|c|c|}
\hline \multicolumn{2}{|r|}{ AUFGABEN DER INTERPRETATIONSHILFE } & \multirow{2}{*}{$\begin{array}{l}\text { BEISPIEL } \\
\text { Kombiniert relevante Aussageebenen um } \\
\text { Zusammenhänge generieren }\end{array}$} \\
\hline$\checkmark$ & $\begin{array}{l}\text { Kombination relevanter } \\
\text { Aussageebenen }\end{array}$ & \\
\hline$\checkmark$ & textliche Kommentare & $\begin{array}{l}\text { Erklärende Beschreibung des jeweils dargestellten } \\
\text { Karteninhalts. }\end{array}$ \\
\hline$\checkmark$ & $\begin{array}{l}\text { weist auf relevante thematische } \\
\text { Zusammenhänge hin }\end{array}$ & $\begin{array}{l}\text { "Im Norden der Stadt gibt es Viertel, in denen es } \\
\text { vergleichsweise wenige Ärzte gibt." }\end{array}$ \\
\hline$\checkmark$ & erklärt dargestellte Inhalte & $\begin{array}{l}\text { "Wo viele hohe, dunkelrote Balken sind, ist die } \\
\text { Konzentration an Ärzten hoch." }\end{array}$ \\
\hline$\checkmark$ & $\begin{array}{l}\text { Aufmerksamkeitserregung für } \\
\text { spezielle räumliche Ausprägungen }\end{array}$ & $\begin{array}{l}\text { "Man erkennt, dass viele Ärzte an großen Straßen } \\
\text { zu finden sind." }\end{array}$ \\
\hline $\mathbf{x}$ & $\begin{array}{l}\text { Keine graphischen Hinweise zu } \\
\text { relevantem Karteninhalt }\end{array}$ & Keine Pfeile, Kreise oder farbliche Hervorhebung \\
\hline$x$ & $\begin{array}{l}\text { Keine technische Verwendungshilfe } \\
\text { zur Erklärung der Funktionen }\end{array}$ & $\begin{array}{l}\text { "Mit einem Klick auf das Pull-Down-Menü können } \\
\text { Sie auch andere Ärztegruppen betrachten." }\end{array}$ \\
\hline $\mathbf{x}$ & $\begin{array}{l}\text { Keine Bereitstellung von } \\
\text { zusätzlichem Hintergrundwissen }\end{array}$ & $\begin{array}{l}\text { "Die Konzentration von Ärzten ist gering da vor } 20 \text { Jahren } \\
\text { rechtliche Rahmenbedingungen geändert wurden." }\end{array}$ \\
\hline $\mathbf{x}$ & $\begin{array}{l}\text { Keine Aufforderungen sich mit } \\
\text { der Karte auseinanderzusetzen }\end{array}$ & $\begin{array}{l}\text { "Nun finden Sie selbst heraus, wie das Nettoeinkommen } \\
\text { in Zusammenhang mit der Verteilung der Ärzte steht." }\end{array}$ \\
\hline
\end{tabular}




\subsection{GLIEDERUNG DER ARBEIT}

Der Umfang dieser Arbeit setzt sich aus fünf Kapiteln zusammen. Kapitel 2 beschreibt den theoretischen Hintergrund, der im Bezug auf die Benützung von komplexen interaktiven thematischen Karten relevant ist. Aus diesem Kapitel werden Hypothesen zur Beantwortung der Forschungsfrage abgeleitet, deren Evaluierungsmethoden im anschließenden Kapitel 3 zum methodischen Vorgehen enthalten sind. Der Usability-Test wird darin als zentrales Element zur Beantwortung der Forschungsfrage beschrieben. Kapitel 4 beschreibt die empirischen Phasen des Usability-Tests mit seinen Inhalten und den daraus resultierenden Ergebnissen. Kapitel 5 diskutiert die Ergebnisse im Bezug auf ihre Relevanz und Gültigkeit und gibt einen Ausblick in zukünftige Forschung. Abbildung 1-2 zeigt den schematischen Aufbau dieser Arbeit mit ihren wesentlichen Inhalten.

\section{EINLEITUNG}

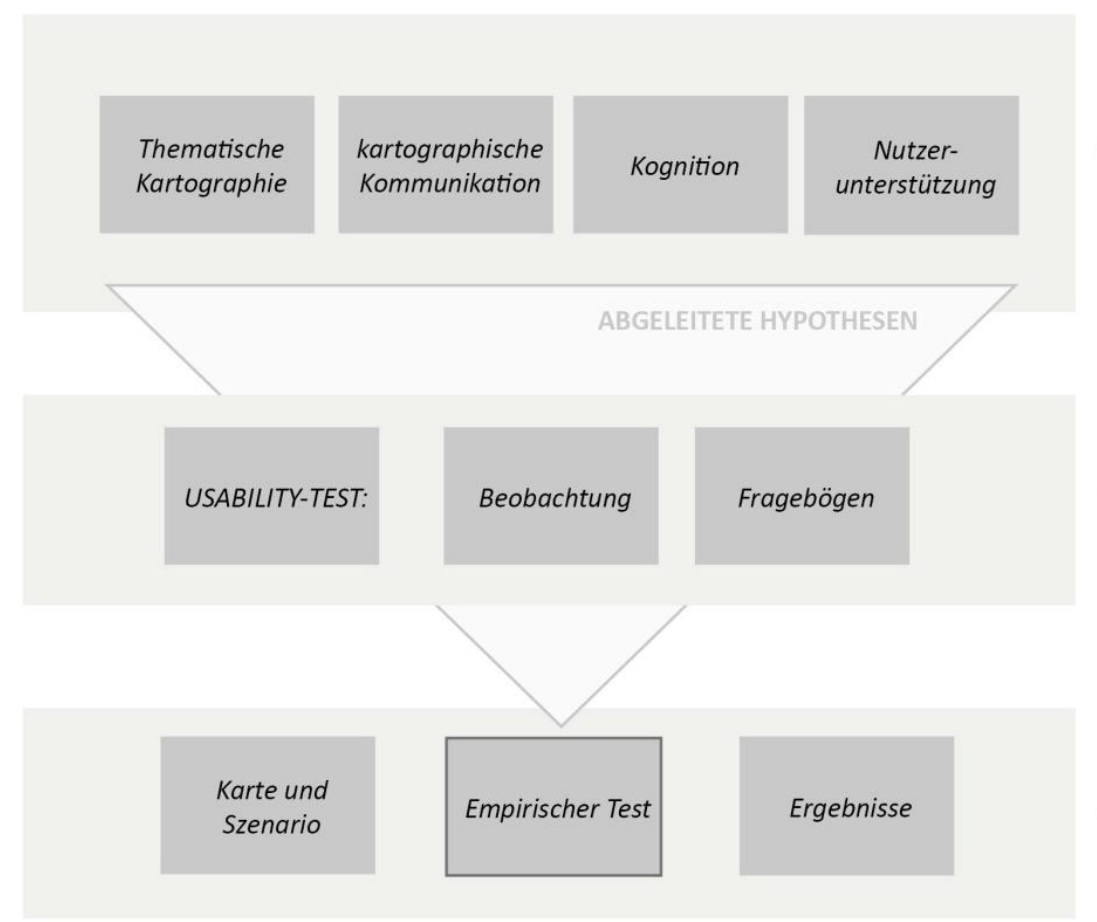

\section{METHODISCHES VORGEHEN




\section{WISSENSCHAFTLICHE GRUNDLAGEN}

Um die in Kapitel 1.2.2 aufgeworfene Forschungsfrage 'Ist eine Interpretationshilfe eine geeignete Methode um die Benützung von komplexen interaktiven thematischen Karten zu unterstützen?' zu beantworten, werden als Grundlage jene Themen theoretisch aufgearbeitet, die mit der Benützung von Karten in Zusammenhang stehen.

Dazu wird zunächst die KIT-Karte in Kapitel 2.1 in die bestehende Terminologie der Kartographie eingeordnet. Darin werden ihre Eigenschaften im Zusammenhang mit weiteren in der Kartographie üblichen Kartendarstellungen beschrieben. Weiters werden in Kapitel 2.2 die Grundzüge der kartographischen Kommunikation erläutert. Diese geben Aufschluss über die einzelnen Schritte, die bei der Kommunikation des von den Kartographen wahrgenommenen Sachverhalts an die Kartenlesenden zum Tragen kommen. Kapitel 2.3 über die kognitiven Aspekte der Kartographie ist ein wichtiges Kapitel, dass eine Auswahl von kognitiven Prozessen anführt, die bei der Verwendung von kartographischen Produkten bedeutend sind. Kapitel 2.4 führt weiters bestehenden Konzepten zur Benutzerführung und Benutzerkontrolle an. In diesem werden Konzepte, die zur Nutzerführung in kartographischen Produkten eingesetzt werden aufgezeigt sowie allgemeine Hilfssystemen in Softwareanwendung angeführt, die in unterschiedlichen Zusammenhängen zur Unterstützung der Nutzenden angesetzt werden.

Dieses theoretische Kapitel bildet die Grundlage zur Ableitung der Forschungshypothesen, die für die Beantwortung der Forschungsfrage herangezogen werden. Am Ende jedes Abschnittes wird auf die zu testenden Hypothesen verwiesen.

\subsection{THEMATISCHE KARTOGRAPHIE UND KIT-KARTEN}

In der Literatur können Karten(-sammlungen) mit Möglichkeiten zur Nutzerinteraktion je nach verfügbaren Funktionen beispielsweise als 'interaktiven Karten' oder als 'Kartographische Informationssysteme' beschrieben werden. Interaktive Karten beschreiben allgemein alle jenen Karten, in denen eine wechselseitige Beziehungen zwischen Karte und Kartennutzendem bestehen (Cammack 1999). Dieser Begriff ist dahin sehr allgemein, der nicht festlegt, über welche Inhalte in der Karte dargestellt 
werden. 'Kartographische Informationssysteme' sind Informationssysteme, die neben Funktionen zur Informations- und Wissensvermittlung auch über Funktionen zur Kartenherstellung und Datenverwaltung verfügen. Durch sie können Nutzende Klassenbildung, Auswahl von Zeichen, Farbwahl und Konstruktion von verschiedenen Kartentypen bestimmen (Kelnhofer 2000). Kartographische Informationssysteme verfügen daher über umfassende Funktionen, die weit über eine reine "Nutzung" von Karten hinausgehen.

Diese Arbeit befasst sich mit der Benützung von Karten, die über sehr spezielle Eigenschaften hinsichtlich Inhalt und Interaktionsmöglichkeiten verfügen. Dieser Umstand erfordert es, die in der Literatur verwendeten Begrifflichkeiten weiter zu spezifizieren um sicherzustellen, dass durch den verwendeten Begriff alle notwendigen Eigenschaften abgedeckt werden. Ausgehend davon wird der neue Begriff 'KIT-Karte' eingeführt. 'KIT' steht hier als Abkürzung für komplex, interaktiv und thematisch.

In diesem Kapitel wird nun beschrieben, wie der Begriff 'KIT-Karte' in Bezug zu bestehenden Konzepten der Kartographie zu setzen ist. KIT-Karten werden dabei in Hinsicht auf komplexe, analytische und synthetische Karten, sowie auf Interaktivität, Datenvisualisierung und Infografik beschrieben.

\section{a) Komplexität}

In der Literatur besteht bereits der Begriff der 'komplexen Karte'. Er beschreibt eine thematische Karte, in der mehrere Variablen meist mit sachlichem Zusammenhang (z.B. Temperatur und Niederschlag) wiedergegeben werden. Es handelt sich dabei um sogenannte polythematische Karten, in denen mehrere Themen behandelt werden, die dabei einzeln sichtbar bleiben. Komplexe Karten stellen eine Zusammenschau mehrerer analytischer Karten dar, einer weiteren Kategorie thematischer Karten (Hake, Grünreich und Meng 2002; Olbrich, Quick und Schweikart 2014). Analytische Karten sind jene Karten, die nur einen einzelnen Betrachtungsgegenstand darstellen z.B. nur Niederschlag oder nur Temperatur (Imhof 1972).

Die Definition der KIT-Karte erweitert die Bedeutung der komplexen thematischen Karte, da ihre Komplexität in verschiedenen Bereichen begründet ist. Sie erfüllt einerseits die Ansprüche einer komplexen Karte, da unterschiedliche thematische Elemente überlagert werden. Dabei können die verschiedenen Signaturschichten (beispielsweise flächige Aussage der Choropletenkarten mit Lokalsignaturen) durch interaktive Elemente ein- und ausgeblendet werden. Der zweite Bereich, der KITKarten komplex macht ist die Möglichkeit die klassische Kartendarstellung mit weiteren Darstellungsformen (beispielsweise Diagrammen) zu kombinieren. Das erlaubt, dass zusätzlich zur Karte Informationen auf eine andere Art den Nutzenden präsentiert werden können.

Aufgrund der Kombination unterschiedlicher Visualisierungen zu einem Thema weist die KIT-Karte weiters Eigenschaften eines Atlasses auf. Dieser ist, laut Definition, als eine Sammlung von Karten zu sehen, die für ein bestimmtes Gebiet und für eine bestimmte Zielsetzung aufbereitet wurde. Der Atlas verfügt über einen konsistenten Leitfaden, der notwendig für den Vergleich zwischen den verschiedenen Einzelkarten ist. Die Reihenfolge, nach der die Karten im Atlas angeordnet werden, spielt dabei eine wichtige Rolle (Hake, Grünreich und Meng 2002).

Die Komplexität der KIT-Karten, die sowohl innerhalb der einzelnen Kartenvisualisierungen als auch durch den Bezug der einzelnen Kartenvisualisierungen zueinander entsteht, erfordert einen gewissen 
Aufwand der Kartennutzenden, um die Zusammenhänge zu erkennen. Illinsky und Steel (2011) erklären, dass die Möglichkeiten des Gehirns beim Lesen von komplexen Visualisierungen begrenzt sind. Abbildung 2-1 verdeutlicht diese begrenzte Leistungsfähigkeit. Jede Ressource, die für das Entziffern und Lesen der Karte eingesetzt wird, kann nicht mehr für das Verstehen der Karte verwendet werden. Erst wenn die verschiedenen Aussageebenen miteinander in Bezug gesetzt wurden, kann die Karte von den Nutzenden richtig verstanden werden.

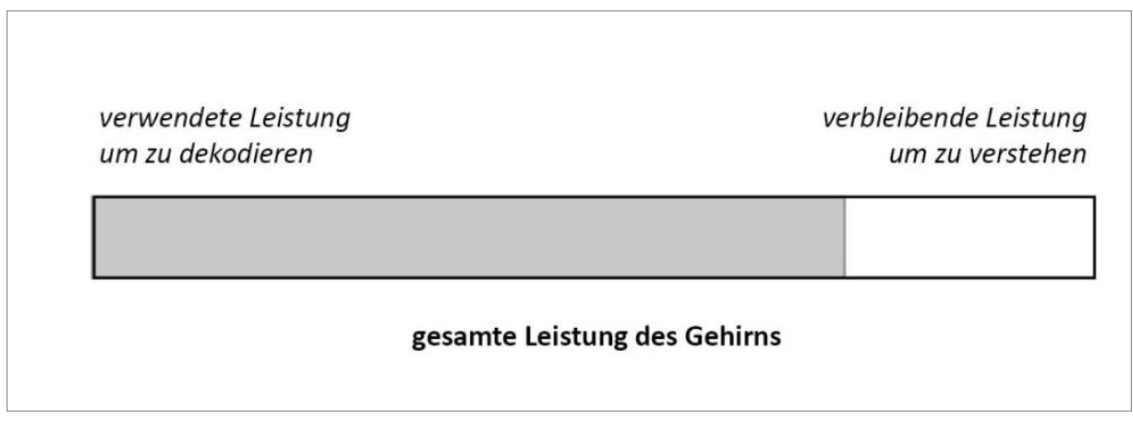

Abbildung 2-1 Begrenze Leistung des Gehirns für Lesen und Verstehen von komplexen Visualisierungen (Iliinsky und Steele 2011, 24)

Der Bezug zwischen verschiedenen thematischen Aussageebenen kann auch durch die Darstellung von 'synthetische Karten' geschehen. Synthetische Karten sind Karten, in denen durch Synthese relevanter Variable zu einem neuen Thema zusammengefügt werden. Meist entsteht daraus eine Typisierung, in die nicht nur statistische Daten sondern auch wissenschaftliches Hintergrundwissen zur Bewertung eingeflossen sind. In synthetischen Karten sind die einzelnen Komponenten meist nicht mehr erkennbar, was eine präzise Erläuterung des neuen Themas in der Legende notwendig macht. (Olbrich, Quick und Schweikart 2014; Imhof 1972). In der KIT-Karte sollen die verschieden Aussageebenen jedoch nicht zu einer synthetischen Karte zusammengefasst werden, sondern durch Interaktivität miteinander in Verbindung gebracht werden.

\section{b) Interaktivität}

Interaktivität ist stark mit der Natur des Internets verbunden, in dem Inhalte von Webseiten durch Links miteinander verbunden werden. Diese Funktion ist heute zu einer selbstverständlichen und notwendigen Sache geworden, die auch von Nutzenden einer Karte erwartet wird (Kraak und Ormeling 2010; Gartner 2000).

In der Literatur werden mehrere Ansätze zur Beschreibung der Charakteristik von interaktiven Karten diskutiert (u. A. Peterson und Sawyer 2008). Gartner (2000) beschreibt als eine wichtige Komponente von Interaktivität die Interaktion zwischen Mensch und Computer bei der auf jede durchgeführte Handlung eine Reaktion folgt. Die Möglichkeit für die Nutzenden mit der Informationspräsentation "umzugehen" (Gartner 2000, 44), ist dabei beschreibend für Interaktivität. Für Kartennutzende ermöglicht die Interaktivität, Inhalt und Präsentation von Informationen zu verändern und so den individuellen Informationsgewinn zur Thematik zu verbessern.

Generell funktionieren interaktive Karten danach, dass zusätzliche Informationen hinter dem Kartenbild verborgen werden und erst durch bestimmte Techniken sichtbar werden. Elemente zur Navigation, Filtern und Suchen von Daten ebenso zum Anklicken, Zoomen oder Mouse-Over-Funktionen 
(Veränderung der Darstellung oder des Inhalts bei Bewegungen der Computermaus) spielen dabei eine Rolle (Kraak und Brown 2001). Diese Funktionen ermöglichen es, viele Daten in einer Karte unterzubringen, die je nach aktuellem Bedarf sichtbar oder nicht sichtbar sind. Sie haben daher im Vergleich zu statischen Karten den Vorteil, mit mehr Datenebenen gefüllt werden zu können.

Neben der Anwendung in KIT-Karten wird Interaktivität auch in vielen weiteren kartographischen Produkten verwendet. Beispielsweise enthalten digitale Atlanten oder sogenannten Karten-Mash$U p s^{1}$ digitale Elemente, die ihre Benützung ermöglichen.

\section{b) thematischer Bezug}

In der Kartographie kann aufgrund des Inhalts einer Karte zwischen topgraphischer und thematischer Karte unterscheiden werden. In topographischen Karten gilt die Darstellung von "Situation, Gewässer Geländeformen, Bodenbewachsung und eine Reihe sonstiger Erscheinungen" als Hauptgegenstand der Darstellung (Internationale Kartograph. Vereinigung 1973 nach Hake 2002). Thematische Karten stellen hingegen ausgewählte geographische Sachverhalte dar und machen ein bestimmtes Thema durch ihre Visualisierung verständlich (Schertenleib und Egli-Brož 2008). Vorwiegend werden Erscheinungen oder Vorkommnisse visualisiert, die nicht topographischer Art sind, jedoch einen räumlichen Bezug aufweisen (University of Zurich 2014). Die kartographischen Visualisierungen der KITKarte sind daher als thematische Karten zu sehen, die Themen mit räumlichem Bezug veranschaulichen. Kartendarstellungen mit überwiegend topographischen Inhalten wie Google Maps zählen aufgrund dieser Definition nicht zu KIT-Karten.

Die KIT-Karte kann daher so wie jede andere thematische Karte durch unterschiedliche Methoden der kartographischen Darstellungen umgesetzt werden. Choroplethenkarten, die Attribute zusammengefasst auf Regionen abbilden; Punktkarten, die räumliche Verteilung auf Basis von Punkten gleicher Größenordnung darstellen oder proportionale Signaturen, die je nach Wert verschiedene Größen aufweisen, können zur Darstellung eingesetzt werden (Imhof 1972). Wie die KIT-Karte, die im Zuge dieser Arbeit getestet wird, umgesetzt wird, beschreibt Kapitel 4.1.1.

\section{c) Kartendarstellung}

Bei KIT-Karten handelt es sich um Karten, die beispielsweise im Gegensatz zu Kartographischen Informationssystemen, mehr auf die Benützung und weniger auf die Erstellung der Karte durch die Nutzenden ausgerichtet sind. In ihrer Erscheinung weisen sie Ähnlichkeiten mit der derzeit populären Darstellungsform von Datenvisualisierungen auf.

Iliinsky und Steele (2011) definierten Datenvisualisierungen (Data Visualization) als Darstellungen, die computergestützt durch einen Algorithmus gezeichnet werden. Sie beinhalten einen großen Umfang an Daten, was als eine der beschreibenden Attribute von Datenvisualisierungen gilt. Eine weitere Charakteristik von Datenvisualisierungen besteht hinsichtlich ihres automatisierten und formalisierten Erstellungsprozesses. Im Allgemeinen ist es einfach die Darstellung mit anderen Daten neu zu

\footnotetext{
${ }^{1}$ In Karten-Mash-Ups werden räumliche Daten aus unterschiedlichen Quellen miteinander kombiniert. Räumliche Daten können dadurch auf bereits bestehenden Kartengrundlagen dargestellt werden, die digital, multimedial und interaktiv den Nutzenden zur Verfügung stehen (Lange 2013).
} 
erstellen bzw. zu aktualisieren, wenn diese in Dimensionen und Charakteristiken einander ähnlich sind. Abbildung 2-3 zeigt ein Beispiel einer solchen Datenvisualisierung.

Im Zusammenhang mit Datenvisualisierung wird auch oft der Begriff 'Infografik' verwendet. Smiciklas (2012) bezeichnet Infografik als eine Methode mit der verschiede Thematiken auf unterschiedliche Weisen abgebildet werden können. Er versteht darunter eine Art von Bild in dem Daten mit Design verbunden werden. Durch visual learning sollen komplexe Informationen und Aussagen dem Zielpublikum schnell, einfach und prägnant präsentiert werden (Smiciklas 2012). Formal definiert Smiciklas die Infografik als "visualization of data or ideas that tries to convey complex information to an audience in a manner that can be quickly consumed and easily understood" (Smiciklas 2012, 1). Die Abgrenzung zwischen Datenvisualisierung und Infografik wird in der Literatur noch nicht ganz einheitlich verwendet. Abbildung 2-4 zeigt ein Beispiel einer Visualisierung, die Eigenschaften einer Infografik aufweist.

Iliinsky und Steele (2011) diskutieren die Unterscheidung dieser Begriffe hinsichtlich der Methode, mit der die Darstellung generiert wird, der Datenmenge, die dargestellt wird und dem Grad der Ästhetik, der angewendet wird. Im Gegensatz zu Datenvisualisierungen werden Infografiken meist händisch durch ein Computerprogramm gezeichnet. Sie wirken dadurch, anders als Datenvisualisierungen, ästhetischer und ansprechender auf die Nutzenden. Infografiken begrenzen sich auch meist auf einen eher geringen Umfang an Daten, da jede Information manuell kodiert werden muss. Weiters sind die Visualisierungen dem jeweiligen Datensatz sehr spezifisch angepasst, was es zusätzlich erschwert, dieselbe Darstellung mit anderen bzw. neueren Daten zu füllen. Abbildung 2-2 visualisiert diese Unterscheidung anhand dieser Kriterien.

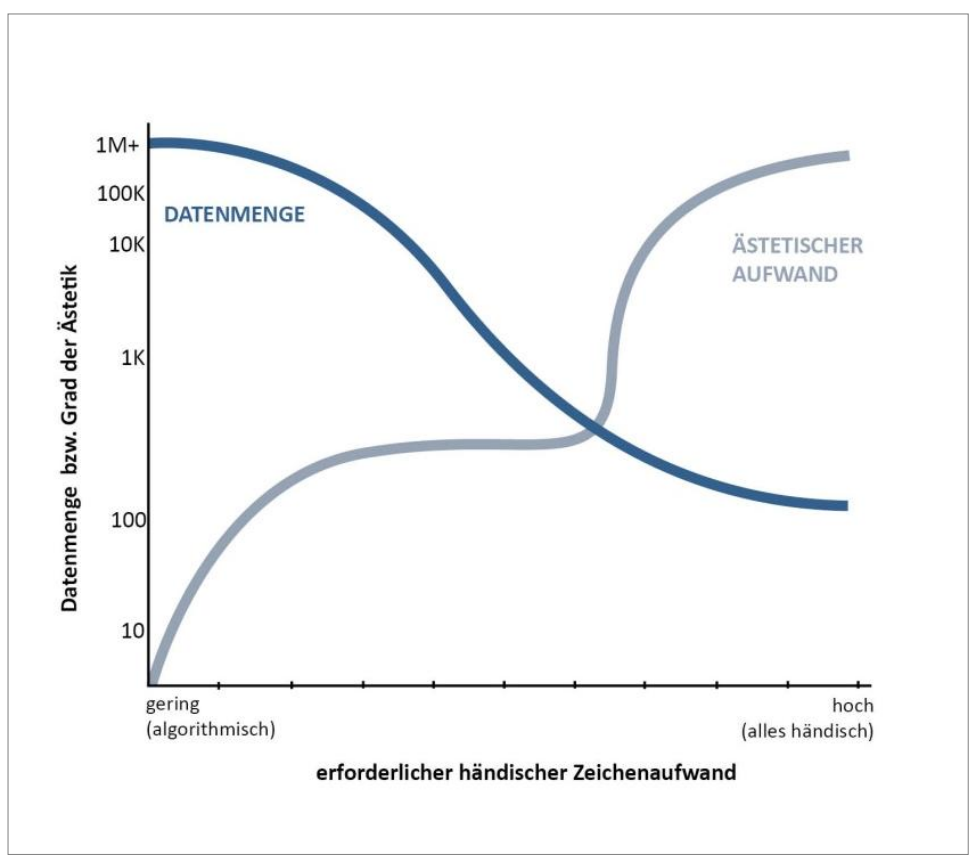

Abbildung 2-2 Unterschied zwischen Infographik und Datenvisualisierung (Iliinsky und Steele 2011, 5) 
Bezugnehmend auf die Definitionen von lliinsky und Steele (2011) lassen sich die KIT-Karten in die Kategorie der Datenvisualisierung einordnen. Der Schwerpunkt von KIT-Karten liegt wie bei Datenvisualisierungen nicht in der anspruchsvollen graphischen Aufbereitung weniger Daten, sondern in der Veranschaulichung von einer großen Menge an räumlichen Daten. KIT-Karten verfügen jedoch über interaktive Elemente, die für Datenvisualisierungen nicht explizit Definiert werden.

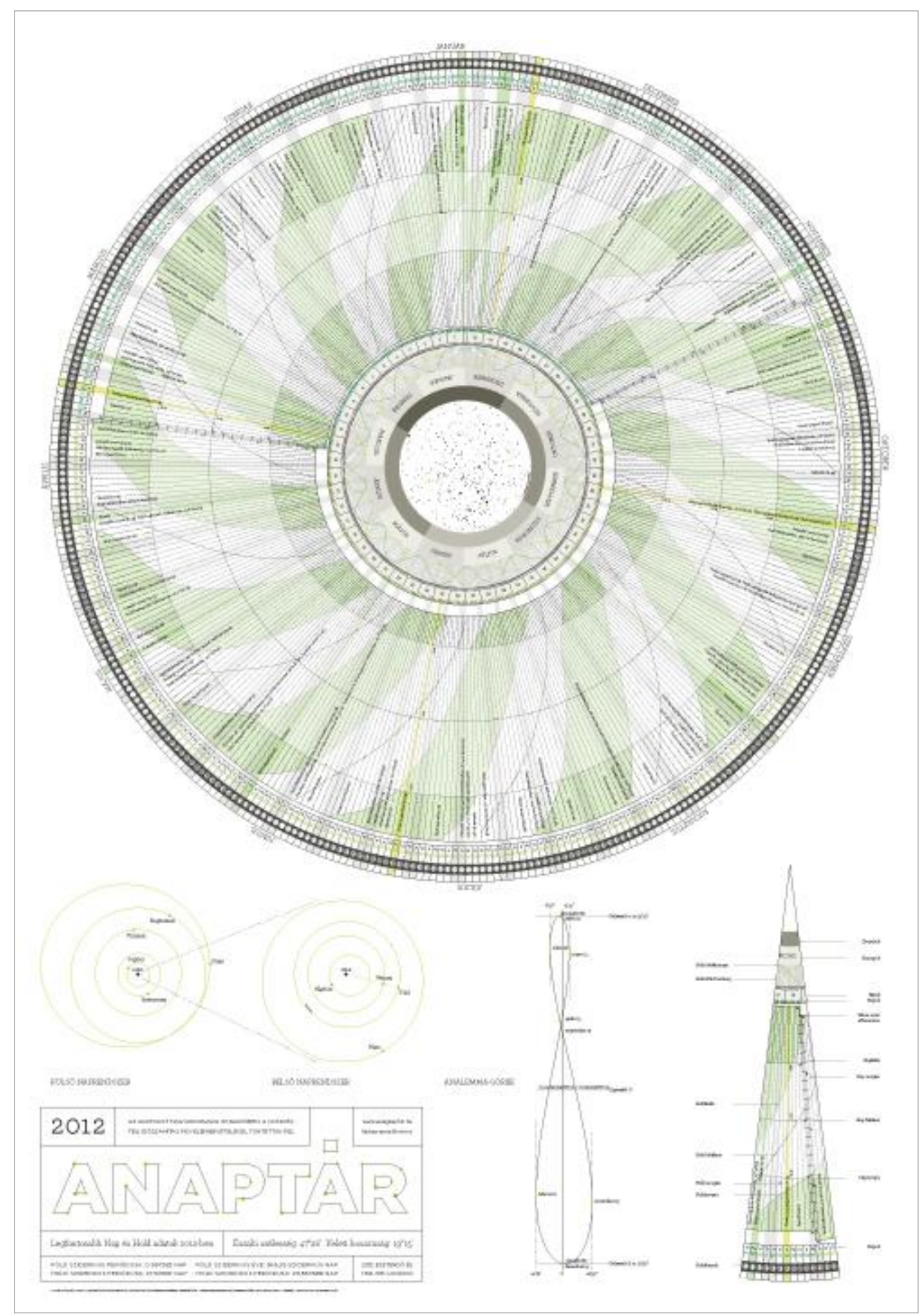

Abbildung 2-3 Beispiel einer Datenvisualisierung mit einer großen Menge an Daten (Sonnen- und Mondsystem, anagraphic 2014) 


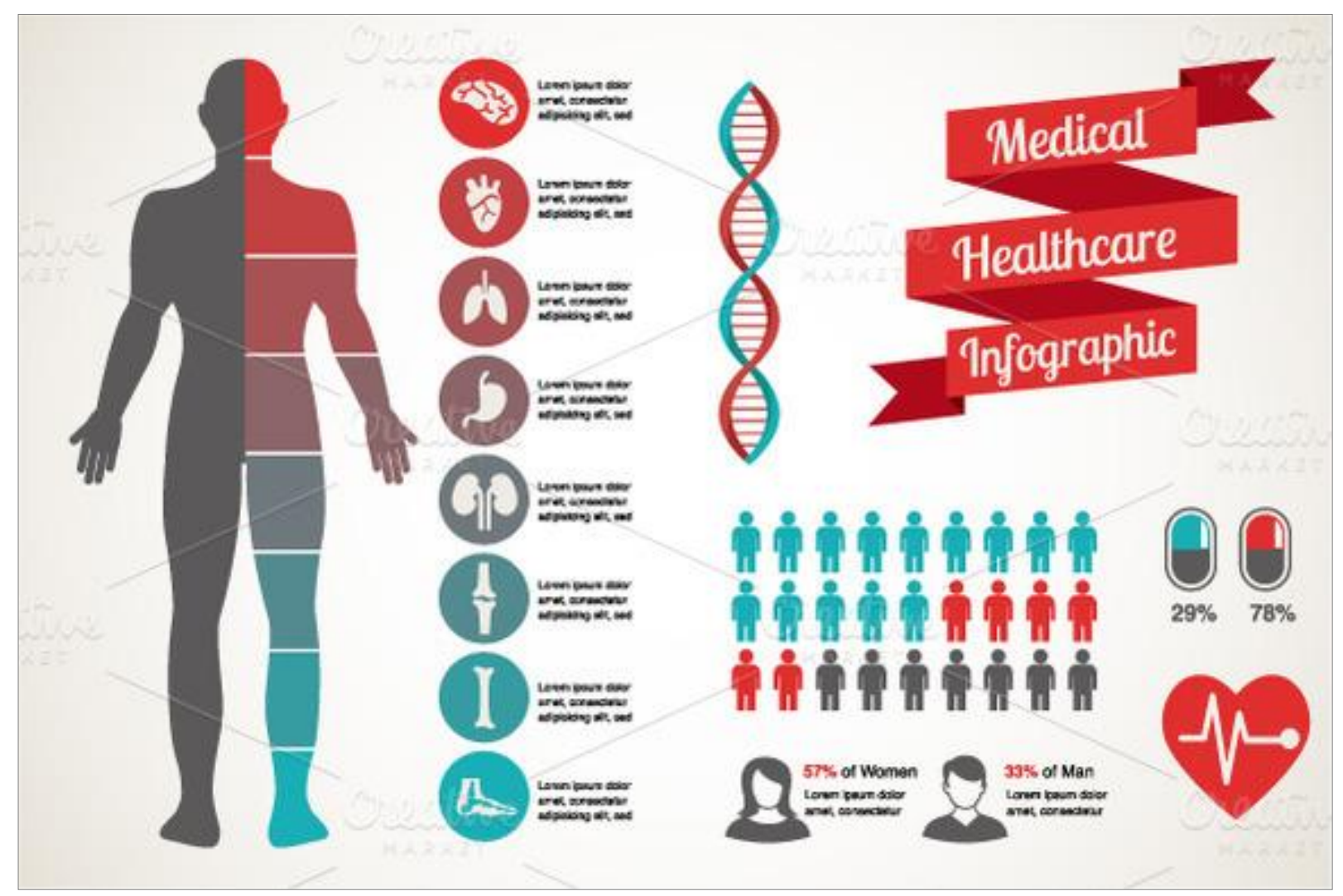

Abbildung 2-4 Beispiel einer Infographik 'Medical Health Care' (www.vectorstock.com)

\subsection{GRUNDZÜGE DER KARTOGRAPHISCHEN KOMMUNIKATION}

Der amerikanische Kartograph Arthur H. Robinson legte in den 1950er Jahren den Grundstein zur Betrachtung der Kartographie als Kommunikationsprozess, die bis heute großen Einfluss auf die kartographische Forschung hat (Ooms 2012 nach Montello und Robinson 2002, MacEachren 2004). Die Informationstheoretiker Claude E. Shannon und Warren Weaver stellten 1949 das Shannon-WeaverModell auf, dass Kommunikation als Prozess mehrerer Schritte definiert (siehe Abbildung 2-5) (Shannon und Weaver 1949). Vereinfacht wird zur Übermittlung einer Nachricht von der Quelle zum Ziel ein Signal in Form von Schall, Bilder, o.ä. vom Sender an den Empfänger gesendet. Bevor die Nachricht jedoch den Empfänger erreicht, wird sie durch äußere Einflüsse gestört und trifft modifiziert bei diesem ein (Intemann 2002). 


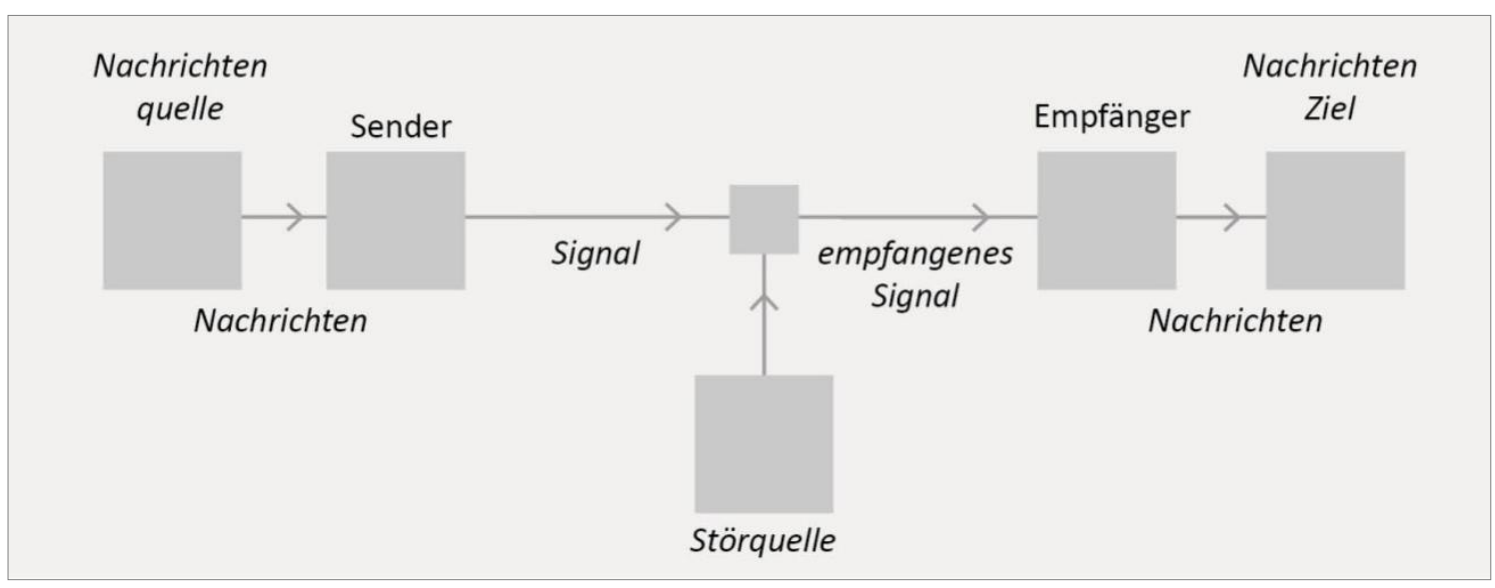

Abbildung 2-5 Kommunikationssystem nach Shannon und Weaver (eigene Darstellung nach Intemann 2002)

Laut Robinsons Theorie gilt in der kartographischen Kommunikation die Karte als Mittel zur Kommunikation von Informationen an Individuen (Issmael und Menezes 2007). Ihre Gestaltung ist daher im Kommunikationsprozess ein wichtiger Qualitätsfaktor und beschreibend für die Wahrnehmung und Interpretation der Information einer Karte (Ooms 2012 nach Montello und Robinson 2002). Beruhend auf Robinsons Ansatz entstand 15 Jahre später die einflussreiche Darstellung des Kommunikationsmodells für kartographische Information vom tschechischen Kartographen Anton Kolácný (1969). Es verdeutlicht weiter die Kommunikation räumlicher Informationen als eine der Hauptaufgaben der Kartographie, in der die Karte als Kommunikationsmittel dient (MacEachren 2004).

Zusammenfassend kann der kartographische Kommunikationsprozess in seiner simpelsten Form als eine Übertragung von Quellinformation zum Empfänger dargestellt werden, in dessen Mittelpunkt die Karte und der Kartennutzende stehen. Die Quellinformation wird aus der uns umgebenden Welt entnommen und an den Kartenleser als Empfänger kommuniziert (siehe Abbildung 2-6). Die in den Karten abgebildeten (räumlichen) Informationen werden kodiert und an die Nutzenden kommuniziert (Ooms 2012). Dies geschieht durch Verwendung kartographischer Symbologien, die beispielsweise darstellen, dass ein größerer Kreis eine höhere Anzahl eines bestimmten Merkmals bedeutet. Die Empfänger lesen die Karte und entwickeln durch Verknüpfung der Informationen mit ihrem Vorwissen ein Verständnis über die Karteninhalte (MacEachren 2004). Ist das Kartendesign nicht optimal, fließen Störungen in den Kommunikationsprozess ein (Montello 2002).

Bei der Verknüpfung vom Dargestellten in der Karte mit dem Vorwissen der Kartennutzenden spielt die Semiotik (Zeichenlehre) eine wichtige Rolle. Semiotik beschreibt wie Texte, Malereien oder andere Medien in ein Zeichensystem zerlegt werden um in Kombination mit anderen Zeichen, die in der Darstellung abgebildet sind, und Zeichen, die kulturell präsent sind, Bedeutung zu finden (Hullman und Diakopoulos 2011). Nach der 'triadischen Zeichenrelation' von Charles S. Pierce (u. A. Hoffmann 2001) wird unter 'Zeichen' alles verstanden, das zum Zeichen erklärt wird. Zeichen sind selbst keine Objekte mehr, sondern repräsentieren diese und müssen von jemandem interpretiert werden. Besteht hingegen zwischen dem Kartenersteller und dem Kartenlesenden kein übereinstimmendes Repertoire an Zeichen, kann die kodierte Information nicht in ihrem Sinngehalt begriffen werden (Lehrstuhl Kartographie 2004). 


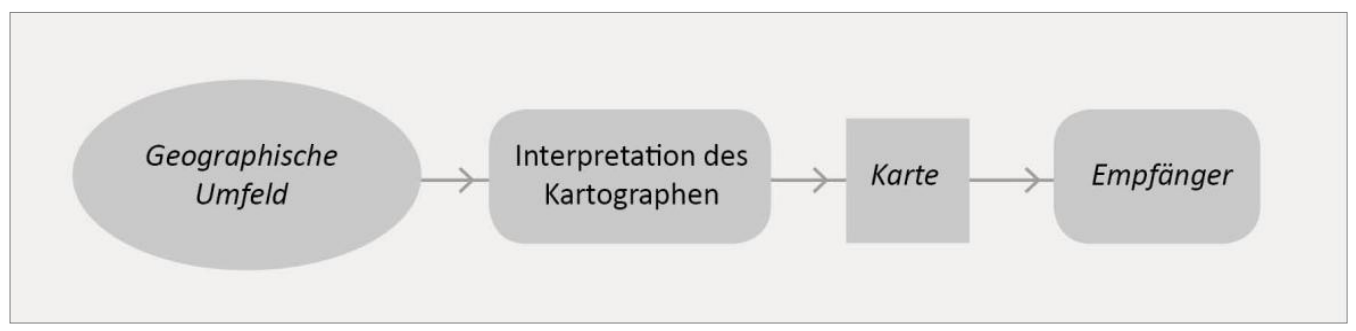

Abbildung 2-6 Kartographie als Kommunikationsprozess von Informationen (eigene Darstellung nach MacEachren 2004,4)

Bei der Übertragung von Informationen an die Kartennutzenden treten mehrere Umwandlungen auf. Einige Autoren identifizieren in diesem Prozess zwei Umwandlungen. Die erste Umwandlung findet von realem Sachverhalt zur Karte statt, die durch den Kartenersteller vorgenommen wird. Die zweite Umwandlung geschieht von der Karte zu einem Bild oder geistigem Modell der Karte, die durch die Kartennutzenden verursacht wird (Issmael und Menezes 2007 nach Blades und Spencer, 1986). Durch die 'Kartographische Informationsverarbeitung' werden weitere Umwandlungen beschrieben. Sie beschreibt den Prozess, bei dem kartographische Informationen in einzelnen Phasen kodiert und dekodiert werden. Zu Beginn werden durch Kartierung und Messung georäumlichen Informationen aufgenommen. Die dabei erfassten Daten werden weiter aufbereitet und verwaltet, um sie in kartographischen Medien abbilden zu können. Aus diesen Abbildungen werden durch visuelle Ableitung Informationen und Wissen gewonnen, die nach einer mentalen Weiterverarbeitung weitere Handlungen ermöglicht. Ausschlaggebend für diese kartographische Informationsverarbeitung ist unteranderem die Verwendung von Kodes. Diese dienen zum kodieren und dekodieren von Informationen bei der Übertragung zwischen Quelle und Ziel. Während dieser Informationsübertragung können sowohl Verluste aber auch Gewinne von Informationen entstehen. Als Übertragungskodes können beispielsweise Schriftsprachen, numerische Systeme oder Bildkodes genannt werden (Lexikon der Kartographie und Geomatik 2014)

Nach dem Modell von Kolácný (1969) zur kartographischen Kommunikation wird von den Kartographen ein kleiner Ausschnitt der Realität beobachtet und kommuniziert (siehe Abbildung 2-7). Die Kartennutzenden verwenden ihr Wissen über die kartographische Sprache, um die Karte zu entschlüsseln und die dargestellte Wirklichkeit nachzubilden. In seinem Modell wird die Kartenerstellung sowie der Interpretationsprozess allerdings von einer Vielzahl von Faktoren beeinflusst. Beispielsweise spielen Vorwissen, Erfahrungen, psychologische Prozesse, Ziele, Aufgaben oder andere externen Bedingungen eine Rolle bei der Interpretation der Karte. Aufgrund dieser Einflussfaktoren entstehen Störungen, die die Nachbildung der Realität des Kartennutzenden nicht mit der (originalen) vom Kartographen beobachten Wirklichkeit übereinstimmen lassen (Ooms 2012). 


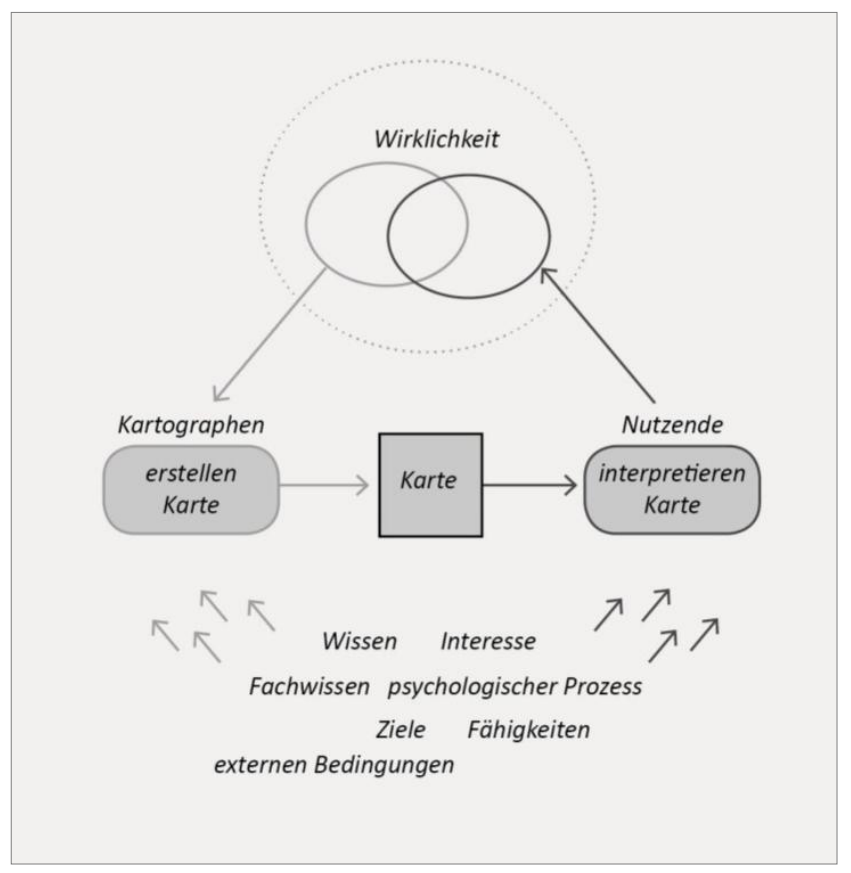

Abbildung 2-7 Modell der kartographischen Kommunikation nach Kolácný (1969) (eigene Darstellung nach Ooms, 2012, 2)

Auch von MacEachren (2004) werden einzelne Hindernisse und 'Filter' beim Kommunikationsprozess identifiziert, die bei der Informationsübertragung von der Realität zum Kartographen, zur Karte und durch die Karte zu den Kartennutzenden auftreten können. Auf der Seite der Kartographen können Hindernisse aufgrund von bestimmten Zielsetzungen, Wissen und Erfahrung, Einstellungen und Fertigkeiten als auch externen Anforderungen, wie Nutzeranforderungen und Abstraktionsprozesse wie Projektion, Generalisierung, Klassifizierung und Symbologie auftreten. Bei der Benützung der Karte auf Nutzerseite kommen Wahrnehmungs- und räumliche Fähigkeiten des Kartennutzers, Verständnis über die Symologie, Ziele, Einstellungen, Zeit zur Betrachtung, Intelligenz und Vorwissen sowie vorgefasste Meinungen zum Tragen. Diese Hindernisse auf beiden Seiten können zu Informationsverlust oder Fehlern in der Kommunikation führen und sollten im Hinblick auf eine verbesserte Kommunikation reduziert werden.

MacEachren (2004) führt weiter fort, dass die Anwendung des Kommunikationsmodells jedoch bei manchen Kartographen umstritten ist. Diese argumentieren, dass nicht jede Karte mit dem Gedanken produziert sei, eine bestimmte Botschaft zu übertragen. Auch aus thematischen Karten, bei denen man von einer eindeutigen Botschaft ausgehen könnte, können je nach Fragestellung unterschiedliche Informationen gewonnen werden. Die Kritiker des Kommunikationsmodells sehen die Übertragung der Informationen weniger als eine 'Botschaft', die von Kartographen gesendet wird, sondern vielmehr als eine Information, die von den Nutzenden aufgrund der kartographischen Darstellung konstruiert wird. MacEachren beschreibt die Disziplin der Kartographie als viel mehr als ein Kommunikationsmodell. Die Disziplin der Kartographie verlange nach Untersuchungen im Bereich von Wahrnehmungs- und kognitiver Prozesse beim Kartenlesen.

Um eine funktionierende Kommunikation der Informationen an die Kartennutzenden sicherzustellen, sind kognitive Aspekte zu beachten. Im anschließenden Kapitel werden nun jene kognitiven Aspekte beschrieben, die bei der Benützung einer Karte bedeutend werden. 


\subsection{KOGNITIVE ASPEKTE IN DER KARTOGRAPHIE}

Dass Karten Einfluss auf das Denken der Mensch hat, ist Kartographen vermutlich schon seit einigen Jahrhunderten bekannt. Dennoch erlangte kognitive Forschung im Zusammenhang mit Kartendesign erst seit dem 20. Jahrhundert verstärkt Bedeutung. Bis zu diesem Zeitpunkt wurde Kartographie hauptsächlich unter ihren handwerklichen, historischen und mathematischen Gesichtspunkten betrachtet und weniger nach der wissenschaftliche Frage, wie Menschen Karten sehen und interpretieren, behandelt (Montello 2002).

Dieses Kapitel setzt sich mit dem Begriff 'Kognition' im Bezug zu Kartographie auseinander. Wahrnehmung, Gedächtnis, Aufmerksamkeit und Verstehen sind einige der wichtigsten Komponenten, die Einfluss auf die Kartenverwendung haben. Im Folgenden wird nun für jeden dieser Bereiche die kartographische Relevanz erläutert und festgestellt, welchen Einfluss die Verwendung einer Interpretationshilfe auf diesen haben kann.

\subsubsection{KOGNITION ALS BEGRIFF}

Der Begriff 'Kognition' leitet sich vom lateinischen cognoscere für 'lernen' und 'wissen' ab. Im Allgemeinen zählen Prozesse wie Denken, Erinnern, Lernen, Tagträumen, Entscheidungen treffen, Sehen, Lesen, Schreiben, Sprechen ebenso wie Aufmerksamkeit, Wahrnehmung/Perzeption, Gedächtnis, Schlussfolgern, Sprechen, Zuhören, Lösen von Problemen oder Planen zu den verschiedenen Arten der Kognition (Rogers, Sharp und Preece 2011).

Im Zusammenhang mit der Disziplin der Kartographie ermöglichen es kognitive Prozesse, Sinn aus einem visuellen Bild abzuleiten (MacEachren 2004). Für die Erstellung und Verwendung von Karten spielen demnach neben Kenntnissen in der jeweiligen Anwendungsdomäne eine Vielzahl von psychologischen Erkenntnissen und Methoden eine wichtige Rolle. (Sarodnick und Brau 2011). Sarodnick und Brau (2011) nennen als Ziel der kognitiven Forschung Technik auf die Fähigkeiten der Nutzenden abzustimmen. Sie verweisen darauf, dass Wissen in diesem Bereich eine Grundlage zur Gestaltung von Benutzeroberflächen darstellt. Der Begriff 'Kognitive Kartographie' verdeutlicht den engen Zusammenhang zwischen Kartographie und Kognition speziell. Der Begriff entstand im Zuge von Studien zu kartographischer Kommunikation und ihrer Verbindung zur Kognitionstheorie. Kognitive Theorien und Methoden werden angewendet um Karten zu verstehen und die Kommunikation zwischen Kartenleser und Karte effektiver zu gestalten (Issmael und Menezes 2007).

Die wichtige Rolle der Kognitionstheorie in der kartographische Kommunikation wird nicht nur von MacEachren (2004) belegt, sondern auch in den von Ooms (2012) zusammengefasst Arbeiten von Harrower (2007), Slocum et al. (2001) und Montello (2002, 2009). In diesen Arbeiten werden die kognitiven Prozesse der Nutzenden mit 'effektiver Kartennutzung' in Verbindung gebracht. Effektive Karten beachten den Umstand, dass nicht nur die Aufbereitung einer Karte (map design) ausschlaggebend für eine effektive Verwendung ist, sondern auch wie die Nutzenden die dargestellten Informationen verarbeiten. Als 'effektive Karten' können jene bezeichnet werden, deren Inhalt leicht von 
den Nutzenden verarbeitet werden kann, obwohl sie komplexe Informationen enthalten. Die Studien von Harrower (2007) und Slocum et al. (2001) identifizieren vor allem für sehr dynamische und interaktive Karten Herausforderungen im Zusammenhang mit effektiven Karten. Bei diesen müssen die Grenzen der Kartennutzenden und ihre kognitiven Verarbeitungsfähigkeiten (processing capabilities) besonders beachtet werden.

Das Verständnis über die Nutzenden und wie sie die präsentierten visuellen Informationen speichern und weiterverarbeiten ist essentiell in der kartographischen Kommunikation (Ooms 2012). Segel und Heer (2010) weisen allerdings darauf hin, dass neben rein kognitiven Faktoren auch andere Faktoren Einfluss auf die Wahrnehmung und Interpretation von Darstellungen haben können. So können kulturelle Faktoren, wie beispielwiese die Richtung in der Gelesen wird, bestimmend sein, wie Darstellungen betrachtet werden.

Im Folgenden werden die wichtigsten kognitiven Prozesse, die beim Kartelesen involviert sind, behandelt.

\subsubsection{PERZEPTION UND WAHRNEHMUNG}

Perzeption befasst sich damit, wie Informationen der Umgebung durch die Sinnesorgane Augen, Haut (beispielsweise Finger) und Ohren erfasst werden und wie diese in Erfahrungen von Objekten, Geschehen, Geräuschen und Geschmack transformiert werden. Bei sehenden Menschen gilt Sehen als wichtigster Sinn, der gefolgt wird von Hören und Berühren (Rogers, Sharp und Preece 2011). MacEachren (2004) beschreibt die Sehwahrnehmung als einen Prozess, bei dem durch ein komplexes informationsverarbeitendes System eine Abfolge von Abbildungen generiert wird. An unterster Stufe steht die Abbildung der visuellen Szene auf der Retina des Auges. Diese Abbildung wird durch eine Reihe von neurologischen Vorgängen zu einer stimmigen Beschreibung der visuellen Szene weiterverarbeitet. Diese Beschreibung kann in höherrangigen kognitiven Prozessen abgefragt werden.

Sarodnick und Brau (2011) weisen auf den von Preim (1999) und Anderson (1996) angeführten grundlegenden Unterschied bei visueller Wahrnehmung nämliche dem fovealen und peripheren Sehen, hin. Foveales Sehen bezeichnet die Reizaufnahme mit einem kleinen zentralen Bereich der Netzhaut (etwa 1-2 Grad), in dem mit hoher Auflösung scharf gesehen wird. Im verbleibenden Bereich nimmt die Schärfe und Auflösung nach Außen hin immer weiter ab. In diesem Bereich des peripheren Sehens können trotzdem Reize entdeckt werden, die die Augenbewegung steuern. Die aufgenommenen Reize werden anhand der von Wertheimer und Köhler aufgestellten Gestaltgesetze zu schlüssigen Objekten zusammengesetzt (siehe Abbildung 2-8). Kriterien dafür sind das 'Gesetz der Nähe', bei dem nahegelegene Elemente als zusammengehörig empfunden werden, das 'Gesetz der Ähnlichkeit', das ähnlich aussehende Objekte gruppiert, das 'Gesetz des glatten Verlaufs', bei dem Linien so wahrgenommen werden, dass sie keine Ecken erzeugen und das 'Gesetz der Geschlossenheit bzw. guten Gestalt', bei dem Objekte als überdeckend wahrgenommen werden um in geschlossenen, klaren Formen zu resultieren. Anschließend wird das zu Objekten Zusammengefasste über Anwendung der Mustererkennung weiter identifiziert.

MacEachren (2004) und Rogers et al. (2011) bringen vor, dass Wissen über die Wahrnehmung und ihre Grenzen essentiell bei der Auseinandersetzung mit dem Lesen von Karten ist. Erst durch Wissen 
über Wahrnehmung und ihre Grenzen kann der komplexe Prozess des Kartenlesens richtig interpretiert werden.

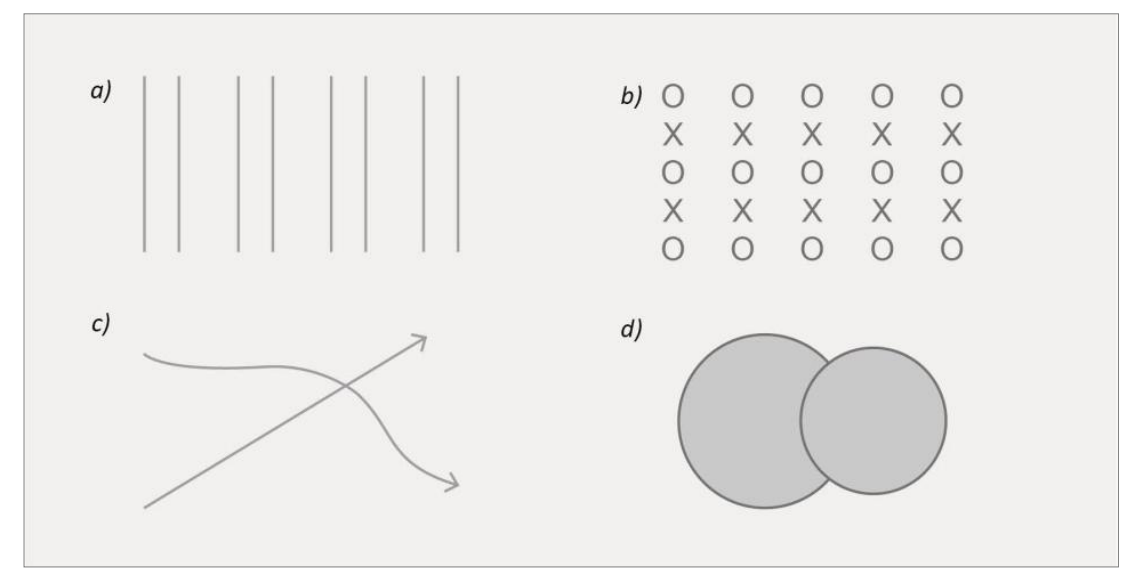

Abbildung 2-8 Die Gestaltgesetze nach Wertheimer und Köhler (eigene Darstellung nach Sarodnick und Brau 2011,53)

a) Gesetz der Nähe, b) Gesetz der Ähnlichkeit,

c) Gesetz des glatten Verlaufs, d) Gesetz der Geschlossenheit

\subsubsection{GEDÄCHTNIS}

Das Gedächtnis umfasst das Abrufen von verschiedenen Arten von Wissen, das auf Sehen, Hören, Schmecken, Riechen oder Berühren begründet wird. Um das Gehirn vor Überforderung zu schützen, die Eintreten würde, wenn alle gewonnenen Eindrücke gespeichert würden, wird ein Filterungsprozess eingesetzt. Dieser entscheidet, welche Informationen vom Gehirn weiterverarbeitet und gemerkt werden. Dabei werden auch Dinge vergessen, an die man sich eigentlich gerne erinnern würde und umgekehrt. Während des Filterungsprozesses wird zu Beginn eine Kodierung festgelegt, die entscheidet, welchen Informationen Aufmerksamkeit geschenkt wird und wie diese verarbeitet werden. Das Ausmaß der Aufmerksamkeit fördert die Weiterverarbeitung der Information durch Denken und vergleicht diese mit anderem Wissen. Je mehr Aufmerksamkeit einer bestimmten Sache geschenkt wird, desto besser kann sich später an sie erinnert werden (Rogers, Sharp und Preece 2011).

\subsubsection{AUFMERKSAMKEIT}

In dem von Ooms (2012) geschafftem Überblick zu den kognitiven Prozessen der Kartennutzenden wird der enge Zusammenhang zwischen Aufmerksamkeit und der Interpretation von Karten vorgebracht. Dabei wird Aufmerksamkeit als "concentration of mental activity" (Ooms 2012, 101) definiert die auf Objekte gerichtet werden müssen, um diese zu interpretieren. Wohin die Aufmerksamkeit des Nutzenden (Focus of Attention) gerichtet wird, bestimmt welche Elemente registriert werden. Ooms (2012) verweist neben Rensink (2002) und Wolfe (1994) auch auf Hegarty et al. (2010), die festgestellt haben, dass das Aufmerksamkeitsverhalten der Nutzenden durch die Gestaltung der Elemente auf einer Karte beeinflusst werden kann (Ooms 2012).

Hegarty et al. (2010) zitieren weiters als eines der grundlegendsten Merkmale der menschlichen Wahrnehmung die Begrenztheit des Aufmerksamkeitsvermögens. Reale Objekte oder Objekte auf Bildschirmen konkurrieren miteinander um die Aufmerksamkeit der Nutzenden. 
Rogers et al. (2011) beschreiben 'Aufmerksamkeit' als jenen Prozess, bei dem von einer Vielzahl von zur Verfügung stehenden Möglichkeiten für einen bestimmten Zeitpunkt, jene Dinge selektiert werden, auf die sich konzentriert wird. Die dabei involvierten Sinne sind Hören und Sehen. Durch Aufmerksamkeit kann sich auf jene Information konzentriert werden, die für die aktuelle Situation bedeutend ist. Wie gut dieses Konzentrieren funktioniert, hängt einerseits davon ab ob ein bestimmtes Ziel verfolgt wird und andererseits ob die notwendige Information aus der Umgebung hervorsticht. Sarodnick und Brau (2011) erklären, dass Elemente durch Farbe, Größe und Anordnung von ihrer Umgebung hervorgehoben werden können, um die Aufmerksamkeit der Nutzenden zu gewinnen. Auch Bucher und Schumacher (2006) stellen fest, dass durch optische Hinweise, vor allem in der ersten Phase der Exploration eines Mediums, die Aufmerksamkeit leicht durch optische Hinweise gelenkt werden kann. In diesem Zusammenhang verwenden Hegarty et al. (2010) den Begriff Visual Salience, der Elemente zu beschreibt, die über gewissen Eigenschaften verfügen, um aus ihrer Umgebung hervorzustechen.

Im Folgenden werden die Grundsätze nach denen die Aufmerksamkeitszuwendung in Karten und anderen relevanten Medien erfolgt erläutert. Es werden allgemeine Theorien sowie spezielles Verhalten bei der Benützung des Internets und im speziellen beim Besuch von Webseiten beschrieben.

\subsubsection{Selektive vs. geteilte Aufmerksamkeit}

Aufgrund der limitierten kognitiven Ressourcen der Menschen ist Aufmerksamkeit nicht unbegrenzt und muss je nach Situation verteilt werden. Dabei kann entweder auf einzelne Reize fokussiert werden (selektive Aufmerksamkeit) oder die Aufmerksamkeit auf mehrere Reize gleichzeitig gerichtet sein (geteilte Aufmerksamkeit). Sarodnick und Brau (2011) beschreiben, dass zwischen diesen zwei Möglichkeiten schnell gewechselt werden kann. Beispielsweise kann beim Autofahren von geteilter Aufmerksamkeit, die viele Reize der Umgebung wahrnimmt, im nächsten Moment schnell auf selektive Aufmerksamkeit umgeschaltet werden, um richtig auf eine Situation zu reagieren. Der Cocktailparty-Effekt beschreibt hingegen, dass bei selektiver Aufmerksamkeit, die einem Gesprächspartner entgegengebracht wird, trotzdem wahrgenommen werden kann, wenn am anderen Ende des Raumes der eigene Name fällt.

Bei visueller Aufmerksamkeit, wie sie beim Lesen von Karten benötigt wird, werden die kognitiven Beschränkungen schneller sichtbar als beispielsweise bei akustischer Aufmerksamkeit. Zu einem Zeitpunkt kann nur eine Stelle bewusst wahrgenommen werden. Wie bereits in Kapitel 2.3.2 angesprochen, können allerdings auch Dinge am Rande des Sichtfelds bemerkt und die Aufmerksamkeit samt Sichtfeld auf dieses neue Ziel gerichtet werden (Sarodnick und Brau 2011).

\subsubsection{Locus of Attention}

Raskin (2000) beschäftigt sich in seiner Forschung damit, wie Gesehenes wahrgenommen wird. Er unterscheidet zwischen dem, was bewusst und unbewusst gesehen wird und verdeutlicht, dass bloßes Sehen nicht bedeutet, dass dieses auch im Gedächtnis bleibt. Dieser Umstand wird durch den Begriff Locus of Attention definiert. Locus bedeutet Platz oder Ort und beschreibt ein Merkmal bzw. Objekt der physischen Welt oder eine Idee, an die aufmerksam und bewusst gedacht wird. Da der Mensch aufgrund der Grenzen des kognitiven Bewusstseins (limitations of cognitive conscious) nicht mehreren gleichzeitigen Stimuli Aufmerksamkeit entgegenbringen kann, kann der Locus of Attention nur auf eine Sache gerichtet werden. Worauf dieser gerichtet ist, kann vom Menschen selbst nicht 
vollständig kontrolliert werden. Wird beispielsweise nach einem Gegenstand gesucht, der, obwohl er sich innerhalb des Sehfeldes befindet, nicht gefunden wird, kann dieses durch den Locus of Attention erklärt werden (Raskin 2000). Weiters erklärt Raskin, dass wenn Aufmerksamkeit auf einen bestimmten Locus gerichtet wird, die restliche Umgebung nicht bewusst beobachtet werden kann. Raskin erklärt diesem Umstand anhand des Beispiels eines guten Magiers. Dieser schafft es, die Aufmerksamkeit seines Publikums auf eine Hand zu fixieren, sodass kein einziger Zuschauer sieht, was die andere Hand macht, obwohl diese in keiner Art versteckt ist.

Raskin (2000) verweist in seiner Arbeit auf Baars (1988), der in diesem Zusammenhang feststellt, dass Nutzende, die einer anspruchsvollen Information folgen, alternative Informationen, die zur selben Zeit für dasselbe Sinnesorgan präsentiert werden, nicht bewusst wahrnehmen. Raskin führt weiter fort, dass bei intensiver Beschäftigung mit einer Information, andere Ereignisse exkludiert werden. Weiters wird es bei steigender intensiver Konzentration auf eine Sache umso schwieriger, den Locus of Attention auf andere Bereiche zu verlagern, da der Stimulus, der diese Veränderung herbei führen soll, umso größer sein muss. Simons (2000) führt weiter aus, dass wir Menschen intuitiv annehmen, dass markante Objekte automatisch unsere Aufmerksamkeit gewinnen. Das ist überraschenderweise oft nicht der Fall und tritt vor allem bei Objekten ein, die nicht erwartet werden. Dieses Phänomen wird als inattentional blindness beschrieben und steht in Verbindung mit der Hypothese, dass wir Menschen uns dem Grad der Blindheit gegenüber unbeachteten und unerwarteten Stimuli nicht bewusst sind. Es wird angenommen, dass wichtige Ereignisse automatisch die Aufmerksamkeit, von dem was gerade getan wird, weglenken.

\subsubsection{Navigationsverhalten auf Webseiten}

Auf Webseiten wird die Aufmerksamkeit und Informationsverarbeitung der Nutzenden maßgeblich durch ihr Navigationsverhalten beeinflusst. Dieser Zusammenhang wurde durch Studien zum Thema 'Bannerblindheit' verdeutlicht. Der Banner wird im Internet zu Werbezwecken eingesetzt, um durch einen Hyperlink auf die Webseite des Werbenden zu verweisen. Bannerblindheit beschreibt das Phänomen, bei dem bestimmte Elemente auf einer Webseite von den Nutzenden nicht wahrgenommen werden, obwohl sich diese durch Größe, Farbe und Animation eindeutig vom Rest der Seite abheben (Kalbach 2008).

Pagendarm und Schaumberg (2006) fassen zwei unterschiedliche Typen des Navigationsverhaltens der Nutzenden zusammen und stellen diese in den Zusammenhang mit der Wahrnehmung von Bannern auf einer Webseite. Zum einen kann das Navigationsverhalten einer zielgerichteten Suche entsprechen. Zielgerichtetes Suchen tritt auf, wenn die Nutzenden nach einer bestimmten Information, wie einem Preis oder einer Telefonnummer, suchen. Die Aufmerksamkeit wird auf jenen Inhalt fokussiert, von dem erwartet wird, dass dieser relevante Informationen für den jeweiligen Zweck enthält. Die Aufmerksamkeit wird bewusst auf die Bereiche der Webseite gerichtet, wo relevante Information erwartet werden und wird demzufolge von einem Top-Down-Prozess gelenkt. Dabei werden die Nutzenden von kognitiven Schemata geleitet, die als wichtige Hilfsmittel gelten, um dem Gesehenen Bedeutung zuzuordnen. Findlay et al. (1995, nach Montello 2002) beschreiben dieses selektive Fokussieren der Informationsverarbeitung auf bestimmte Bereiche eines Bild als visual Attention, die von den kognitiven Schemata beeinflusst wird. Haben Nutzende viel Erfahrung im Umgang mit dem Internet wissen sie, dass relevante Information üblicherweise nicht in Bannern zu finden ist und ignorieren diese. 
Zum anderen kann Navigationsverhalten auch der Charakteristik einer ziellosen Suche entsprechen. Diese beschreibt das Bewegen durch die Information ohne konkreten Plan ein bestimmtes Objekt zu finden. Bei dieser Art des Suchens wird die Aufmerksamkeit der Nutzenden durch einen Bottom-UpAnsatz gelenkt. Nutzende werden bei diesem Ansatz nur durch den Reiz der Information geleitet und reagieren auf jene Stimuli, die ihre Aufmerksamkeit zuerst wecken. Durch die angesprochenen animierten Banner wird die Aufmerksamkeit der Nutzenden unbewusst geweckt und ihre Aufmerksamkeit anschließend auf diese gerichtet. Das Resultat der Studie zeigt, dass Nutzende, die nach keinen bestimmten Informationen suchen, Banner eher wahrnehmen, da sie durch die Reize der einzelnen Elemente auf der Webseite geleitet werden.

Das Verhalten von Nutzenden auf Webseiten wurde vor allem in Zusammenhang mit Bannern, die zu Werbezwecken eingeblendet werden untersucht. Aufgrund dieser zwei gegensätzlichen Grundvoraussetzungen gibt es sowohl Studien, die die Existenz der Bannerblindheit belegen und andere, die sich für Effektivität der Banner für Werbezwecke ausschreiben (Benway 1998). Pagendarm und Schaumburg (2006) merken dazu an, dass sich das Phänomen der Bannerblindheit nicht ausschließlich durch zielgerichtetes und zielloses Nutzerverhalten erklären lässt. Sie nennen Ergebnisse, die darauf verweisen, dass das geringe Anklicken von Bannern auch schlichtweg daran liegen kann, dass die Nutzenden an dem angepriesenen Produkt nicht interessiert sind. Benway (1998) führt bei seiner Interpretation der Ergebnisse an, dass obwohl der sogenannte Werbeeffekt Nutzende dazu leiten kann, werbeähnliche Elemente aufgrund von Erfahrungswerten auszublenden, das Phänomen hervorstehende Elemente zu ignorieren als weitaus größer zu betrachten ist.

\subsubsection{Aufmerksamkeitsspanne im Internet}

Auch für den Wirtschaftsjournalist und Autor Nicholas Carr stellt Aufmerksamkeit einen entscheiden Faktor für den Umgang mit Informationen, die in unserer Welt vorhanden sind, dar. Carr (2011) begegnet dieser Thematik durch eine Untersuchung über Auswirkungen, die durch die Verwendung des Internets auf unser Gehirn entstehen. Er bezieht sich auf eine neurologische Studie die feststellt, dass der Mensch heutzutage nach immer mehr Informationen, mehr Eindrücken und mehr Komplexität verlangt. Situationen, in denen ständig Leistungen abverlangt werden oder in denen man von Informationen überwältigt wird, werden gesucht. Carr sieht im Internet ein Medium, das die Aufmerksamkeit auf sich zieht, diese aber gleichzeitig zerstreut. Er kommt zur Erkenntnis, dass sich unsere Denkweise auf das Überangebot an Informationen im Internet bereits angepasst hat. Die Nutzenden beschäftigen sich intensiv mit dem Medium selbst, werden allerdings durch von miteinander wettstreitenden Nachrichten und Stimuli abgelenkt. Ihm zufolge wurden Medienprodukte bereits an die kürzeren Aufmerksamkeitsspannen der Online-Nutzenden angepasst. Kritisiert wird der, seiner Meinung nach, unhinterfragt gefolgte Grundsatz "je mehr Eindrücke desto besser" (Carr 2011, 46). Der Autor vertritt die Meinung, dass die Aufmerksamkeitsteilung, die durch Einsatz von Multimedia entsteht, unsere kognitiven Fähigkeiten sehr beansprucht und weist darauf hin, dass weniger mehr sein kann (Carr 2011).

Weinreich et al. (2008) führten eine Studie zur Aufmerksamkeitspanne von Internetnutzenden durch. Die Ergebnisse der Studien zeigen, dass Testpersonen Webseiten nur für kurze Zeit aufrufen. Mehr als die Hälfte der Seiten waren nur weniger als zehn Sekunden geöffnet. In einem Viertel der Fälle betrachteten die Teilnehmenden diese weniger als vier Sekunden lang. Allerdings wurden $10 \%$ der Seiten länger als zwei Minuten angezeigt. Er kommt zum Schluss, dass Surfen im Internet als eine 
schnelle Aktivität zu bezeichnen ist, die auch für Webseiten zutrifft, die mit durchschnittlich 551 Wörtern und 53 Hyperlinks beachtlichen Inhalt aufweisen. Es ist offensichtlich, dass niemand innerhalb weniger Sekunden die Inhalte in voller Länge lesen kann, sondern vielmehr nur ein flüchtiger Blick auf die Informationen geworfen werden kann. Weinreichs Ergebnisse untermauern vorangegangene Studien, die ebenfalls ein schnelles Verhalten bei Interaktion im Internet feststellten (Weinreich u. a. 2008). Cockburn und McKenzie (2001) stellten beispielsweise bereits fest, dass viele Leute viele Seiten in einer sehr kurzen Zeit besuchen. Ihre Ergebnisse zeigen, dass zwischen dem Besuchen einer Seite und dem Besuch der Darauffolgenden, meist nur eine Sekunde vergeht. Abstände zwischen den Besuchen mit mehr als zehn Sekunden waren selten zu beobachten (Cockburn und McKenzie 2001). Weiters wird durch Weinreichs Ergebnisse die Aussage von Nielsen (1997) bestätigt, in der er auf die Frage wie Nutzende das Internet lesen, feststellt: "They don't." (Carr 2011,48).

Die kurzen Aufmerksamkeitsspannen müssen auch bei der Erstellung von Karten beachtet werden. Während Weinreich (2008) nach einem Seitenlayout fordert, das auch oberflächliches Lesen erlaubt, erkennen Kraak und Brown (2001), dass die relativ kurze Aufmerksamkeitsspanne der typischen Internetnutzenden Grenzen an den dargestellten Inhalt stellt. Die derzeit sehr populäre Darstellungsform von Datensätzen durch Infografiken bzw. Datenvisualisierungen kann als eine mögliche Antwort auf diese Umstände gesehen werden, auf die in Kapitel 2.1 eingegangen wird. Smikilas (2012) beschreibt in diesem Zusammenhang Infografiken als Darstellungen, die der Zeit von Informationsüberflutung und verkürzten Aufmerksamkeitsspannen begegnen, da sie schnell Information übermitteln können.

\subsubsection{Satisficing}

Ein weiteres Phänomen, das im Zusammenhang von kurzer Aufmerksamkeitsspannen im Internet betrachtet werden kann, wird von Kalbach (2008) genannt. Er bezieht sich auf das sogenannte Satisficing, einem Kunstwort, das 1957 von dem Sozialwissenschaftler Herbert A. Simon geprägt wurde. Der Begriff setzt sich aus satisfy (zufriedenstellen) und suffice (genügen) zusammen und beschreibt die Tendenz, in einer bestimmten Situation zur Erfüllung eines Zwecks nicht nach der optimalen Möglichkeit zu suchen, sondern mit der erstbesten Lösung zufrieden zu sein. Im Zusammenhang mit der Nutzung des Internets in der Praxis erklärt Krug (2006) dieses Verhalten unter anderem dadurch, dass die Nutzenden meist in Eile sind und das 'Raten' im Vergleich zu 'Abwägen' von Optionen weniger Zeit kostet und folglich effektiver ist. Kalbach (2008) vermutet allerdings, dass es sich bei den von Krug (2006) getätigten Aussagen um Verallgemeinerungen handelt. Er sieht das Verhalten der Nutzenden durch Inhalt und Motivation bestimmt und sieht den Besuch einer fachspezifischen Webseite im Vergleich zum Aufrufen einer Shopping-Webseite als etwas, das über bloßes Satisficing hinausgeht.

\subsubsection{Zusammenfassung}

Die in diesem Kapitel angeführten Studien zu Nutzerverhalten und Aufmerksamkeitsverteilung beziehen sich vorwiegend auf die Verwendung von Text- und Bild-basierten Webseiten. Es kann trotzdem angenommen werden, dass diese Ergebnisse auch für die Benützung von KIT-Karten bestimmend sind. Diese sind üblicherweise im Internet verfügbar und ähneln durch ihren interaktiven und komplexen Aufbau Webseiten, in denen durch implementierte Hyperlinks einzelne Inhalte aufgerufen werden können. Auch wenn sich die in diesem Kapitel genannten Studien zur Aufmerksamkeitserregung an optischen Techniken, wie herausstechende Farben, direkte Kenntlichmachung durch 
Kreise oder Pfeile bedienen, können auch durch textliche Erklärungen die Aufmerksamkeit der Nutzenden auf bestimmte Bereiche gelenkt werden. Demnach sind Erkenntnisse aus diesem auch für den Zweck dieser Arbeit relevant.

Selektive und geteilte Aufmerksamkeit sowie das Konzept über den Locus of Attention beschreiben, wie die Aufmerksamkeit der Nutzenden innerhalb einer Darstellung gelenkt werden kann. Ausschlaggebend dafür ist, dass die Aufmerksamkeit des Menschen begrenzt ist und sich durch visuelle Reize leiten lässt. Wie das Beispiel des Magiers zeigt, kann durch Handlungen und optische Hinweise der Fokus der Aufmerksamkeit auf bestimmte Bereiche gelenkt werden, die gesehen und infolgedessen interpretiert werden. Das Phänomen der Bannerblindheit verdeutlicht allerdings, dass bestimmte Vorkenntnisse sowie die Intention bei der Verwendung, die Wahrnehmung des Inhalts beeinflussen können. Auch Forschung über Aufmerksamkeitsspannen, Navigationsverhalten auf Webseiten und Satisficing verdeutlichen, dass Nutzende sich oft nur relativ kurz mit einem Medium beschäftigen und dabei die Fülle der Informationen nicht vollständig wahrnehmen.

\subsubsection{VERSTÄNDNIS VON KARTEN}

Kognitive Prozesse und Aspekte der Neurophysiologie, Psychologie, Ergonomie und Kartographie werden beim 'Lesen' einer Karte miteinander verwoben. Visuelle Wahrnehmung ermöglicht es, die Welt um uns zu interpretieren (MacEachren 2004). Besonders das Gedächtnis und das Vorwissen der Nutzenden sind wesentliche Bestandteile um aus Gesehenem Sinn abzuleiten und somit eine Karte interpretieren zu können (Issmael und Menezes 2007). Den Ablauf, wie aus Kartendarstellungen ein Verständnis über den Inhalt abgeleitet werden kann, wird von einzelnen Autoren aus unterschiedlichen Kontexten heraus dargestellt:

Issmael und Menezes (2007) fassen den Prozess der Karteninterpretation als aufeinanderfolgende Reihe kognitiver Prozesse zusammen. Diese involvieren Denkvorgänge, in denen Informationen wahrgenommen, ausgewählt, verglichen, gespeichert und abgerufen werden.

Sarodnick und Brau (2011) beschreiben, dass Informationen auf Grundlage der Wahrnehmung aufgenommen und verarbeitet werden müssen. Dafür ist es notwendig Sprache, Figuren usw. zu verstehen. Weiters müssen die Informationen auch behalten werden, um sich später an sie erinnern können.

Ooms (2012) fasst zusammen, dass als erster Schritt der Karteninterpretation die graphisch kodierte Information, die auf den Karten abgebildet ist, dekodiert wird. Die visuellen Eindrücke werden im sensorischen Gedächtnis aufgezeichnet. Ein Großteil dieser Eindrücke wird nach weniger als zwei Sekunden wieder vergessen. Ein kleiner Teil gelangt jedoch weiter ins Arbeitsgedächtnis, in dem Informationen auch nur bis zu einer begrenzten Kapazität gespeichert werden können. Durch Wiederholen und Lernen können Informationen vom Arbeitsgedächtnis ins Langzeitgedächtnis weitergeleitet werden, dessen Kapazität praktisch unendlich ist.

Ooms verweist in diesem Zusammenhang auf Matlin (2002), die als ersten Schritt des Interpretationsprozesses das Fokussieren der Aufmerksamkeit auf das Objekt sieht. Die Sinnesreize registrierten visuelle Stimuli, die weiterverarbeitet werden müssen. Um Karten mit ihren dargestellten visuellen Informationen zu interpretieren, werden die visuellen wahrgenommen Stimuli mit bestehenden Er- 
innerungen verglichen und dann identifiziert. Vorwissen ist demnach ein wichtiger und notwendiger Faktor des Interpretationsprozesses.

Nach MacEachren (2004) kann der Bedeutungsgewinn aus einer Karte als Prozess verstanden werden. Aus kartographischer Sicht betrachtet kann Wahrnehmung und visuelle Kognition als Informationsverarbeitung dargestellt werden. Der Schwerpunkt dieser Informationsverarbeitung liegt in einer Serie von Darstellungen, die je nach Verarbeitungsstufe verschiedene Abbildungen der Kartenoberfläche darstellen. In niederstufigen Verarbeitungen enthalten die Darstellungen Zeichen und abstrakte Symbole, die aus grundlegenden Elementen der Karten und allgemeinen Informationen über die Elemente und ihre Beziehungen (z.B. Größe, Helligkeit, usw.) abgeleitet werden. Übergeordnete Weiterverarbeitung führt zu einer visuellen Beschreibung der Abbildung aus der vorangegangenen Stufe. Wissensschemata vermitteln zwischen dieser visuellen Beschreibung und lassen schließlich eine Bedeutung aus der Kartendarstellung ablesen.

Hegarty et al. (2010) fassen aktuelle Modelle zum graphischen Verständnis (graphics comprehension) in der Forschung zusammen (u. A. Herrmann und Pickle, 1996). Dabei werden drei Teilprozesse vorgeschlagen, die für das Verstehen graphischer Darstellungen notwendig sind. Hegarty et al. veranschaulichen diesen Prozess an einem Beispiel zur Informationsgewinnung aus Wetterkarten. Um die Abbildungen verstehen zu können, müssen zuerst die optischen Bestandteile (visual features) der Darstellung von den Nutzenden entschlüsselt werden, z.B. Bereiche mit unterschiedlichen Farben. Im nächsten Schritt müssen diese auf die Zusammenhänge die sie vermitteln, abgebildet werden. Daraus kann geschlossen werden, dass Farbe Temperatur bedeutet. Abschließend sind diese Zusammenhänge mit der Abbildung in Bezug zusetzen. Dies ermöglicht es, von einem roten Bereich, der einen Teil einer Landkarte bedeckt, darauf zu schließen, dass die Temperatur an diesem Ort beispielsweise über $30^{\circ} \mathrm{C}$ beträgt. Hegarty et al. schlussfolgern, dass gutes Kartendesign das Ergebnis des Interpretationsprozesses verbessern kann.

Hegarty et al. (2010) verweisen weiters auf einen Ansatz von Freedman und Shah (2002), der sich mit dem Einfluss von bestehendem Wissen auf die Interpretation von Darstellungen befasst. Es besagt, dass beim ersten Kodieren von optischen Elementen einer Darstellung (visual chunks) automatisch Fachwissen und Erwartungen aktiviert werden. Diese bringt Leute dazu eher etwas zu 'sehen', dass sie erwarten, als was tatsächlich gezeigt wird. Hegarty et al. (2010) belegen in ihrer Forschung, dass Fachwissen die Aufmerksamkeit gegenüber aufgabenrelevanter Bereiche am Bildschirm erhöht und deswegen die Augenfixation beim Interpretieren einer Graphik beeinflusst.

Ein weiter Aspekt, der im Bezug zu kognitiven Aspekten der Wissensinterpretation genannt werden kann, wird von Sarodnick und Brau (2011) zitiert. Sie verweisen auf Forschungsergebnisse, die zeigen, dass die Verknüpfung von verbaler mit bildhafter Information dazu führt, Informationen besser behalten zu können.

\subsubsection{ZUSAMMENFASSUNG UND HYPOTHESEN ZU KOGNITIVEN ASPEKTEN}

Kognitive Aspekte beeinflussen das 'Lesen' einer Karte auf verschiedene Weise. Perzeptive Regeln bestimmen den Prozess, nachdem visuelle Inhalte erfasst werden. Die Strukturen des Gedächtnisses bestimmen wie das Gesehene gespeichert wird. Die entgegengebrachte Aufmerksamkeit legt fest, auf welche Dinge man sich konzentriert und in weitere Folge welche Informationen wahrgenommen 
werden. Im Verständnisgewinn sind Denkvorgänge involviert, die aus den gewonnenen Sinneseindrücken Bedeutung ableiten. Dabei wird deutlich, dass die angeführten kognitiven Fähigkeiten des Menschen keine endlosen Ressourcen darstellen, sondern begrenzt sind. Im Zusammenhang mit dem Einsatz von Interpretationshilfen in KIT-Karten spielen die Lenkung der Aufmerksamkeit und die Prozesse, die notwendig sind um ein Verständnis aus der Kartedarstellung abzulesen, eine besonders große Rolle.

Die in Kapitel 2.3.4.1 angeführte selektive bzw. geteilte Aufmerksamkeit ebenso, wie das in Kapitel 2.3.4.2 genannte Konzept Locus of Attention beschreiben, wohin die Aufmerksamkeit der Nutzenden zu welchem Zeitpunkt gerichtet wird. Bezogen auf den Einsatz der Interpretationshilfe kann der Umstand auftreten, dass die Nutzenden so sehr mit ihren Inhalten beschäftigt sind, dass sie hinsichtlich ihrer begrenzten kognitiven Ressourcen andere Inhalte der KIT-Karte nicht wahrnehmen. Umgelegt auf das von Raskin (2000) genannte Beispiel des Magiers, der die Aufmerksamkeit bewusst auf bestimmte Handlungen lenkt, könnten die Nutzenden beim Lesen der Karte sozusagen 'blind' gegenüber dem werden, was von der Interpretationshilfe nicht angesprochen wird. Auch das in Kapitel 2.3.4.3. behandelte Phänomen der 'Bannerblindheit' kann bei der Verwendung von KIT-Karten Bedeutung finden. Dieses Phänomen resultiert aus dem zielgerichteten Navigationsverhalten der Nutzenden auf Webseiten, das als irrelevant eingestufte Inhalte ausblendet. Im Fall der Interpretationshilfe können die Nutzenden beim Lesen der Karte in dem Sinne beeinflusst werden, dass die textlichen Beschreibungen ein gewisses 'Vorwissen' zur Interpretation des Karteninhalts darstellen. Wird die Interpretationshilfe vor der eigentlichen Betrachtung der Kartendarstellung gelesen, könnte dieses Wissen die Nutzenden zu einem bestimmten 'Ziel' hinleiten und auf die Wahrnehmung von Inhalten Einfluss nehmen. Ein weiteres Phänomen, das zu einer eingeschränkteren Interpretation führen kann, ist das in Kapitel 2.3.4.5 behandelte Satisficing. Die Nutzenden könnten wegen der Fülle an präsentierten Informationen die KIT-Karte nur oberflächlich betrachten und folglich hauptsächlich die textliche Erläuterung beachten. Auch der in Kapitel 2.3.5 im Zusammenhang mit dem Bedeutungsgewinn aus Karten vorgebrachte Ansatz von Freedman und Shah (2002) kann auch in Hinblick auf die Interpretationshilfe relevant sein. Die textlichen Kommentare der Interpretationshilfe könnten bei den Nutzenden Erwartungen wecken, die sie dazu bringen, etwas in der Karte zu 'sehen', dass möglicherweise gar nicht gezeigt wird.

Aus den Erkenntnissen dieses Abschnittes wird folgende Hypothese aufgestellt, die die einschränkende Wirkung der Interpretationshilfe überprüfen soll:

Hypothese 1 Die Verwendung einer Interpretationshilfe führt zur Interpretation von bestimmten Inhalten.

Im Gegensatz zu der in Hypothese 1 vermuteten einschränkenden Wirkung der Interpretationshilfe auf die ganzheitliche Interpretation der Karte, zitiert Hegarty et al. in Kapitel 2.3.5 Forschungsergebnisse, die den positiven Einfluss von Vorwissen auf die Interpretation der Karte belegen. Sarodnick und Brau zitierte weiters die positive Wirkung von der Verknüpfung zwischen Text und Bild. Folgende Hypothese soll den positiven Einfluss der Interpretationshilfe testen: 
Hypothese 2 Durch die Verwendung einer Interpretationshilfe werden die dargestellten Inhalte häufiger korrekt interpretiert.

Nachdem in diesem Kapitel die kognitiven Aspekte, die bei der Verwendung einer Karte bestimmend sind, dargestellt wurden, wird im folgenden Kapitel auf Methoden eingegangen, die zur Nutzerunterstützung in (Karten-)Anwendungen verwendet werden können. Sie sollsicher stellen, dass obwohl den kognitiven Fähigkeiten Grenzen gesetzt sind, die Inhalte von den Nutzenden auch in komplexen Darstellungen erfasst werden können.

\subsection{UNTERSTÜTZUNG FÜR NUTZENDE IN (KARTEN-)ANWENDUNGEN}

Wie bereits im Kapitel 2.2 festgestellt, besteht der Zweck der Kartographie in der Kommunikation von geographischen Informationen. Die kartographische Kommunikation hat sich von einer einseitigen Kommunikation zu einer kartographischen Interaktion entwickelt. Die Kartographische Interaktion beschreibt den Dialog zwischen Mensch und Karte, der durch einen Computer ausgeführt wird. Laut Roth (2013) soll diese Betrachtung nicht das klassische Kommunikationsmodell einer einseitigen Konversation ablösen, sondern vielmehr einer Gleichstellung zwischen Mensch und die Karte dienen, in der der jeweils andere beeinflusst werden kann. Möglich wird die kartographische Interaktion durch ein Interface, das es den Nutzenden erlaubt, die Darstellung des Karteninhalts an den Kontext und das spezielle Interesse anzupassen.

Für Roth (2013) ist die Aufbereitung der Karte ausschlaggebend für eine funktionierende kartographische Kommunikation. Diese muss sicherstellen, dass die Nutzenden alle notwendigen Informationen in jener Form aufnehmen können, die es ihnen ermöglicht, ein umfassendes Bild über den dargestellten geographischen Inhalt zu erlangen. Roth stellt dabei einen Bedarf für zusätzliche Orientierungshinweise in dynamischen, mit vielen interaktiven Möglichkeiten ausgestatten Karten fest. Durch die hohe Komplexität dieser können die Nutzenden bei ihrer Benützung leicht überfordert werden und in der Karte "verloren gehen" (Roth 2013, 76).

Um sinnvolles und effizientes Arbeiten mit KIT-Karten sicherzustellen, müssen folglich mehrere Dinge betrachtet werden. Ein wichtiger Aspekt in der Anwendung von interaktiven Medien in der Kartographie ist die Benutzersteuerung. Kapitel 2.4.1 stellt Herangehensweisen vor, die sich mit der Gestaltung von Nutzerfreiheiten bei der Verwendung dieser Produkte auseinander setzt. Von Bedeutung ist dabei vorallem, wie bei dem umfassenden Angebot von geographischen Informationen sichergestellt werden kann, dass alle relevanten Informationen, die zum Verständnis der Karte führen, von den Nutzenden aufgenommen werden können. Betrachtet man die Karte als Softwareprodukt, die als Schnittstelle zwischen Mensch und geographischer Information dient, werden Prinzipien des Interface Designs relevant. Diese umfassen unter anderem neben der Konsistenz des Interface Designs und der Verwendung von Shortcuts auch das zur Verfügungstellen von Hilfefunktionen (Keyes 2002). Hilfe kann auf verschiedenste Weise in Verbindung mit der Anwendung angeboten 
werden und dient dazu, den Nutzenden Unterstützung bei der Anwendungsverwendung zur Verfügung zu stellen. Kapitel 2.4.2 gibt einen Überblick über einige dieser Methoden.

\subsubsection{BENUTZERSTEUERUNG UND -KONTROLLE IN INTERAKTIVEN KARTENPRODUKTEN}

Die Erstellung von kartographischen Produkten ist heutzutage maßgeblich durch die technischen Möglichkeiten bestimmt. Die Kartographen sind bei der Gestaltung ihrer Produkte deshalb mit immer komplexeren Herausforderungen konfrontiert. Bei dynamischen, interaktiven Karten müssen nicht nur wie bisher Entscheidungen über die Verwendung von optischen Variablen zur Darstellung des geographischen Phänomens, getroffen werden, sondern auch welcher Typ von Karte erstellt werden soll und wie viel Kontrolle die Kartennutzenden über diese haben soll (Elzakker und Griffin 2013).

Elzakker und Griffen (2013) identifizieren insbesondere für dynamische Karten, wie auch KIT Karten welche sind, Risiken, dass Kartennutzende bei der Benützung überfordert sein könnten. Die Autoren bringen vor, dass obwohl Kartenlesende vermutlich viel von den präsentierten Informationen auf einer dynamischen Karte mitnehmen, dennoch viele Informationen übersehen werden. Dies sei auch dann der Fall, wenn die Nutzenden genau wissen wonach sie in der Karte suchen. Die Autoren stützen ihre Annahme unter anderem auf eine von Simons und Levin (1998) durchgeführte Studie, die das Thema Change Blindness untersucht. Bei der Studie wurde eine Person, die einen Passanten um eine Wegbeschreibung bittet, während des Gesprächs mittels Trick durch eine andere Person ausgetauscht. Mehr als die Hälfte der Passanten, die die Wegbeschreibungen gaben, bemerkten nicht, dass die Person mit der sie sprachen ersetzt worden ist. Die Autoren sehen dieses Phänomen auch bei dem Einsatz dynamischer Karten, bei der die Kartenlesenden Veränderungen nicht immer gut wahrnehmen können.

Nicht nur allein die Veränderung der Inhalte und ihrer Darstellung stellen die Nutzenden bei der Verwendung von dynamischen Karten vor Herausforderungen. Die Interaktivität in Karten führt charakteristisch dazu, dass sich auf der Suche nach nützlichen Informationen Multiple-Choice-Optionen eröffnen. Dabei müssen Entscheidungen über den einzuschlagenden Weg getroffen werden, wobei jeder dieser Prozesse in einem anderen Pfad durch das Produkt resultiert. Je nach gewähltem Weg wird hinsichtlich der gefundenen Informationen ein jeweils anderes geographisches Bild entwickelt. Dabei besteht die Gefahr, dass in manchen Fällen diese freien Wahlmöglichkeiten dazu führen, dass essentielle Informationen nicht aufgerufen werden und ein unvollständiges Bild über die Aussage der Karte entsteht.

Komplexe Karteninhalte und viele Wahlmöglichkeiten bei der Verwendung eines kartographischen Produkts bringen die Gefahr, dass essentielle Inhalte gar nicht aufgerufen werden. Es ist daher notwendig, sich mit Konzepten auseinanderzusetzen, die sich mit der Benutzerführung in KIT-Karten befassen. Im Folgenden werden Konzepte vorgestellt, die im Zusammenhang mit der Nutzerführung in kartographischen Produkten entwickelt wurden.

\subsubsection{Metaphern zur Kommunikation geographischer Information}

Bei der Darstellung von Wissen jeglicher Art kann der Begriff der Metapher verwendet werden. Als Metapher versteht man die Form, in der die Information an den Nutzenden geliefert wird. Beispielsweise kann dem Internet die Metapher eines Buches mit unendlich vielen Seiten zugeschrieben werden (media.org 2000). Für die Präsentation von geographischen Informationen existieren vielerlei 
Möglichkeiten. Die Übermittlung muss demnach nicht immer durch traditionelle kartenbasierte Formate geschehen, sondern kann durch Bilder, textliche Erzählungen, Skizzen oder Multimedia ersetzt werden (NCGIA 1997). Cartwright (1999) diskutiert in seiner Arbeit den Einfluss des technischen Fortschritts und die veränderten Erwartungen der Nutzenden an die Darstellung von geographischer Information. Er stellt ein Set an Metaphern vor, das die traditionell verwendete Map Metaphor, die Informationen kartenbasiert präsentiert, ergänzen und erweitern soll. Für die Nutzenden sollen dadurch verschiedene Formen des Informationszuganges ermöglicht werden. Insgesamt werden von Cartwright neun Metaphern vorgestellt, um diese Aufgaben zu erfüllen. Im Folgenden wird nun ein Überblick über jene Metaphern gegeben, die Konzepte zur Navigation, Führung und Kontrolle der Nutzenden in kartographischen Produkten beinhalten und demnach im Zusammenhang mit Nutzerführung in KIT-Karten bedeutend sind.

\section{a) The Storyteller}

Die Storyteller-Metapher wird als Option charakterisiert, die es den Nutzenden erlaubt sich eine Geschichte über eine bestimmte Gegend oder Ortes erzählen zu lassen. Die Erzählweise wird durch Interaktivität bestimmt und kann entweder linear oder nicht linear ablaufen. Cartwright (1999) verweist in diesem Zusammenhang auf Platt (1995), der den Nutzenden bei geographical storytelling entweder die Rolle des Beobachter (Observes), des Direktors (Directors) oder des Akteurs (Actors) zu schreibt (siehe Abbildung 2-9). Obwohl es den Nutzendenden vorkommt, als hätten sie die volle Kontrolle über die Geschichte, wird die reale Leitung durch die Autoren des kartographischen Produkts festgelegt. Den Nutzenden bleibt nur die Möglichkeit das Produkt innerhalb der vorgegebenen Grenzen zu steuern.

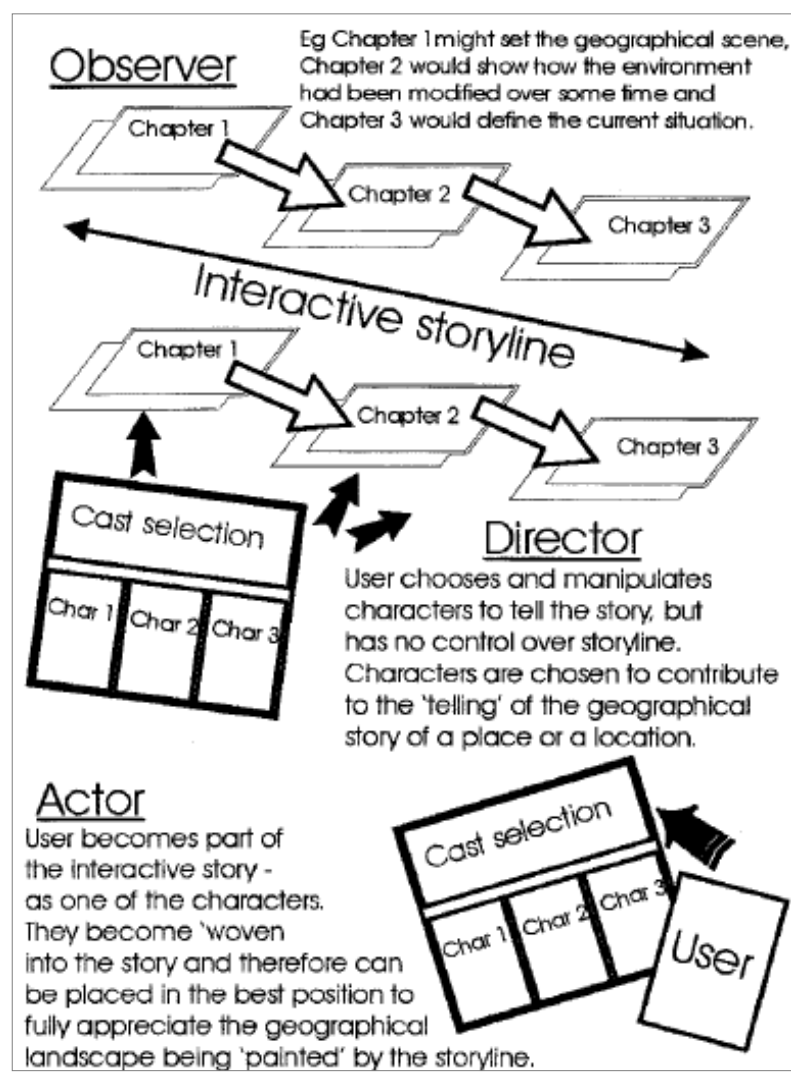

Abbildung 2-9

Platts Idee des 'geographical storytelling'

(Cartwright 1999,338) 


\section{b) The Navigator}

Cartwright (1999) vermutet, dass viele Nutzende von Kartenprodukten keine ExpertInnen bei der Benützung von Karten sind und daher Unterstützung beim Interpretieren von abstrakten Modellen, wie es Kartendarstellungen sind, benötigen können. Eine Unterstützung kann durch die NavigatorMetapher erfolgen. Der Navigator weist auf Stichpunkte oder Landmarks hin, denen die Nutzenden bei ihrer Bewegung durch die Karte begegnen. Diese machen auf Orte in der Karte aufmerksam, die besucht, interpretiert und konsumiert werden sollen, um eine korrekte Interpretation des vorliegenden Systems zu ermöglichen. Abbildung 2-10 zeigt das Konzept der Navigator-Metapher.

Die Navigator-Metapher kann nach Harper (1992) durch unterschiedliche Arten umgesetzt werden: Der Guide, der eine visuelle Reise durch die vorliegende Informationslandschaft ermöglicht; die 'sequenzielle Navigation', die Hinweise zu Orten an denen Information vorhanden sind aufeinander folgend verfügbar macht oder durch die 'visuelle Navigation', die einen Plan über möglichen Pfade durch die Informationslandschaft vorstellt (siehe Abbildung 2-11). Weiters kann die NavigatorMetapher durch die 'hybride Metapher' umgesetzt werden, die geeignete Komponenten der drei genannten Metaphern verbindet. Die Navigationshinweise, die durch den Navigator vorgestellt werden, können die Nutzenden sowohl zu weiteren Informationen innerhalb des Produkts, als auch zu relevanter Information außerhalb des Produkts weiterleiten (Cartwright 1999).

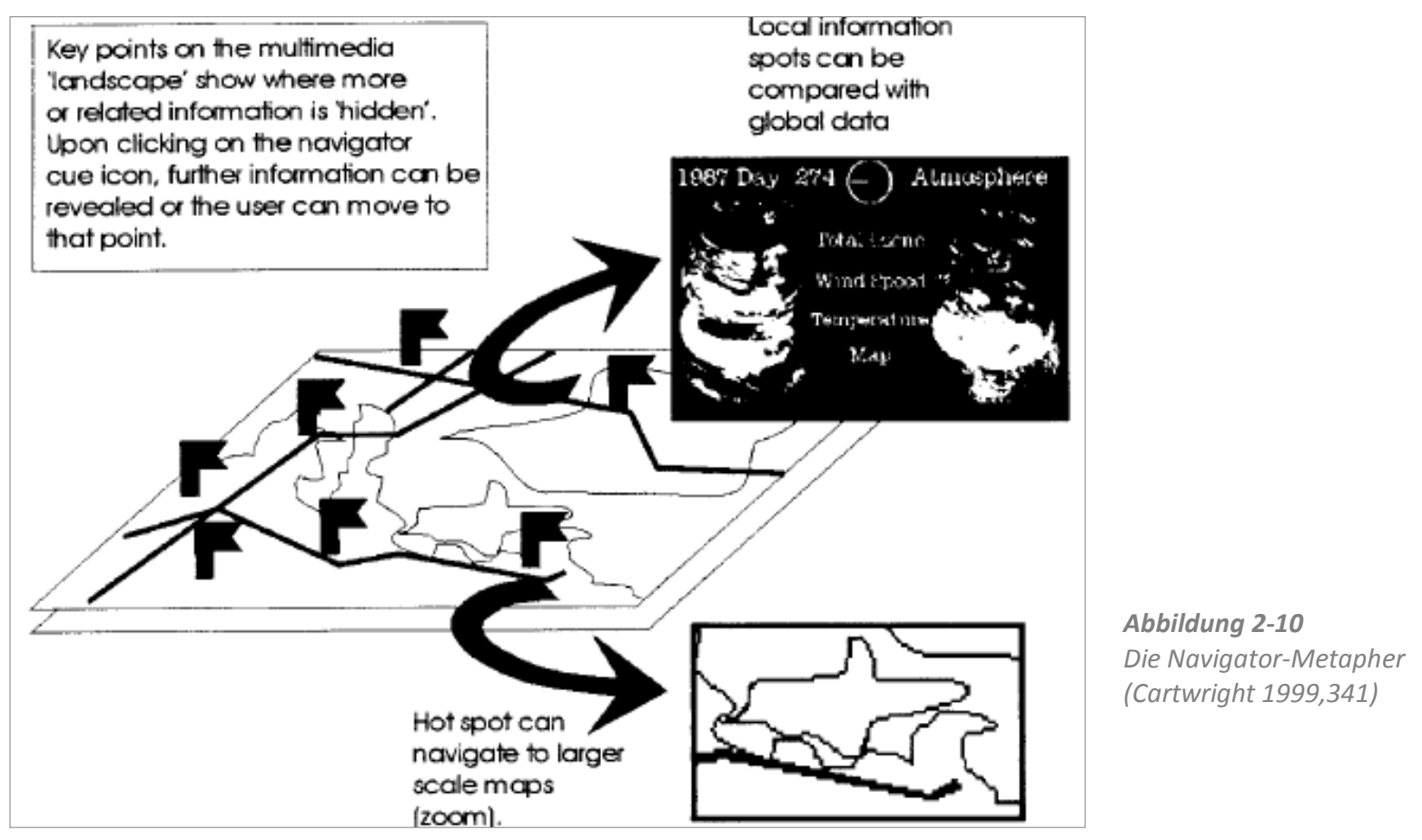

\section{c) The Guide}

Die Guide-Metapher nimmt an, dass die Nutzenden weder Vorwissen über die abgebildete Region noch die Fähigkeit haben, sich effektiv durch die einzelnen Szenen oder das ganze Produkt zu navigieren. Der Guide soll helfen, die dargestellte Information besser wahrzunehmen und zu verstehen. Die Metapher fokussiert die Nutzenden dabei nur auf wichtige Informationen, während irrelevante ignoriert werden. Es wird sichergestellt, dass relevante Informationen automatisch für die Nutzenden 
ausgewählt werden. Der Guide navigiert für die Nutzenden und führt sie zu Orten, für die aufgrund bestimmter Prozesse errechnet wurde, dass sie Informationen enthalten, die notwendig sind, um die geographische Darstellung vollständig zu verstehen (Cartwright 1999) .

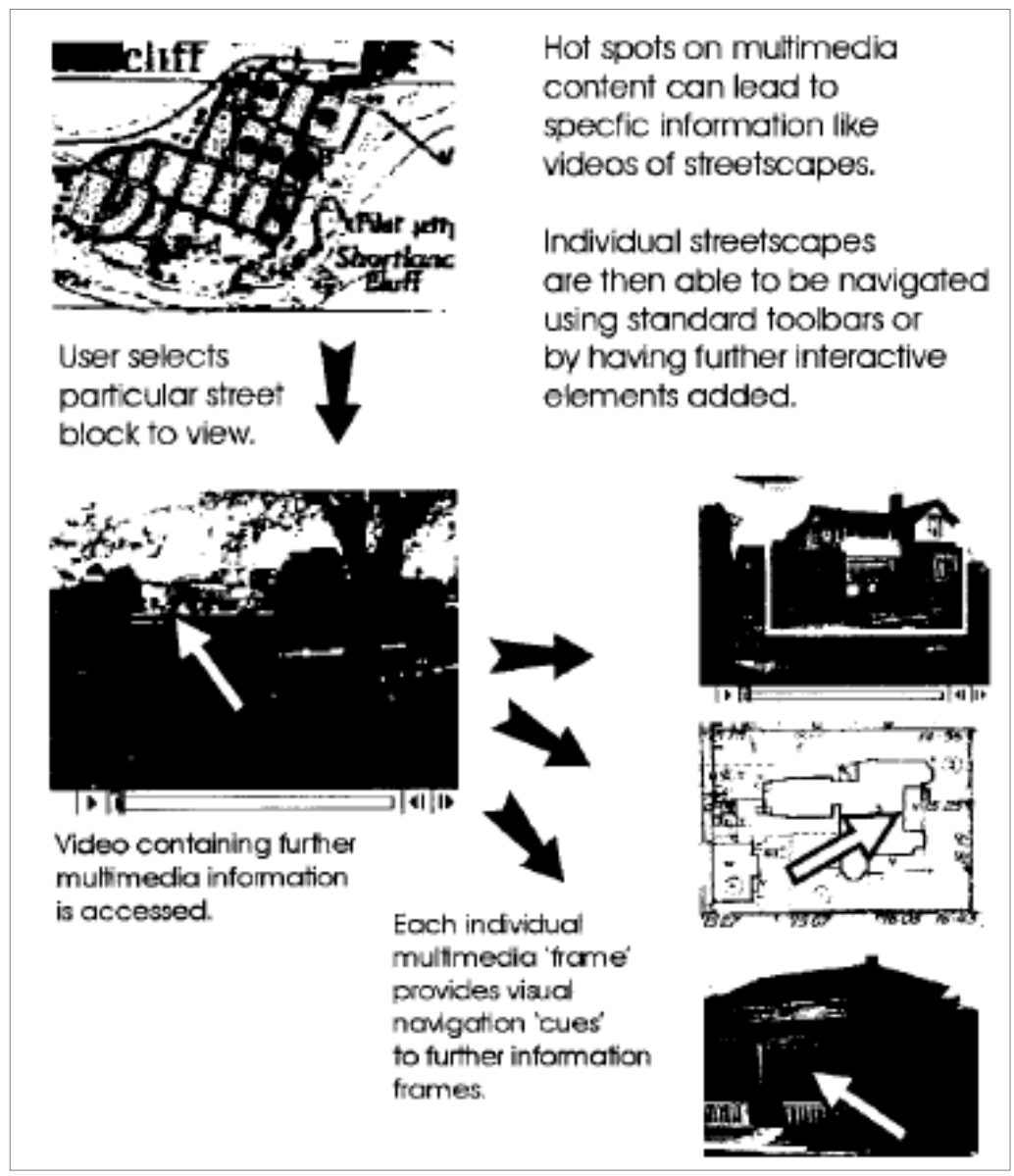

Abbildung 2-11

Beispiel zur Umsetzung von

sequenzieller und visueller Navigation (Cartwright 1999,340)

\section{d) The Gameplayer}

Die Gameplayer-Metapher beinhaltet Spiele, die eingesetzt werden, um geographischen Inhalte zu kommunizieren. Die Umsetzung kann beispielsweise durch einfaches Zuordnen von Fotos oder durch Abrufen von Informationen umgesetzt werden, die neue Zugänge zu geographischen Informationssammlungen bieten. Diese Metapher setzt dabei vor allem darauf, dass die Nutzenden nicht in dem vom Spieldesigner vorgegebenen methodischen Zugang 'eingesperrt' sind, sondern durch Explorieren die Inhalte erfassen und erlernen können (Cartwright 1999).

Die vier dargestellten Metaphern zeigen Möglichkeiten, auf welche Weise geographische Informationen den Nutzenden präsentiert werden können und diese durch die Kartenprodukte geführt werden können. Obwohl die von Cartwright vor mehr als einem Jahrzehnt vorgeschlagenen Metaphern in einigen Punkten überholt wirken können, zeigen sie dennoch den prinzipiellen Grundgedanken, wie die Benutzung einer Karte für die Nutzenden organisiert sein kann. 


\subsubsection{Animation durch Graphic Scripts}

Um ausgeprägte Trends und signifikante Zusammenhänge geographischer Daten zu untersuchen, entwickelt Monmonier (1989) ein Instrument, das er als Atlas Touring bezeichnet. Eine wichtige Komponente dieses Instruments stellt der Einsatz von sogenannten Graphic Scripts dar. Graphic Scripts dienen dazu, geographische Karten, statistische Graphen und Textblöcke zu eine Serie bzw. Animation zusammen zu binden. Die Autoren der Karten setzen dafür einzelnen Sequenzen (Graphic Phrases) zu einer dynamischen Serie zusammen. Durch diese bewegten Bilder wird eine Geschichte über den Datensatz erzeugt. Die Bewegung entsteht durch die Präsentation von Informationen in übereinanderliegenden Ebenen, die mit gleichem Bezug gezeigt werden. Dadurch wird es ermöglicht, raum-zeitliche sowie geographische Trends abzulesen (Monmonier 2011; Fleckenstein 1991; Cartwright 2004).

Den Nutzenden ist es allerdings nicht möglich, in der Animation der Graphic Scripts zu interagieren. Sie werden vielmehr anhand eines Drehbuchs von einer Information zur nächsten bewegt, sicherstellend dass keine essentielle Information ausgelassen wird (siehe Abbildung 2-12). Diese Vorgehensweise gibt den Entwicklern die größtmögliche Kontrolle über die Nutzenden, da diese nicht mehr darüber entscheiden können, wie sie sich durch die Informationen bewegen möchten (Cartwright 2004).

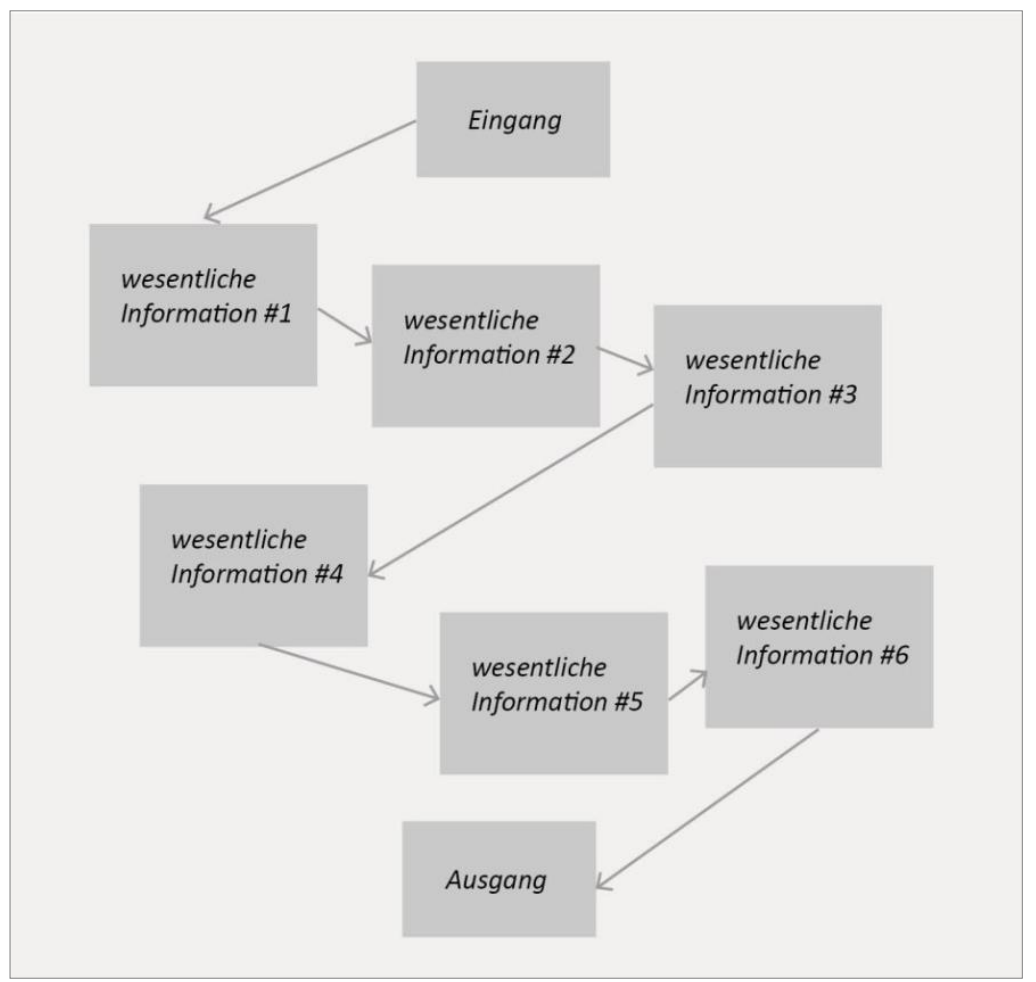

Abbildung 2-12

Das von Monmonier entwickelte Konzept der 'Graphic Scripts' (Cartwright 2004, 6)

\subsubsection{Engineered Serendipity in Kartenprodukten}

Cartwright (2007) behandelt in seiner Arbeit die Diskrepanz zwischen zwei grundsätzlichen Möglichkeiten wie Nutzende eine Karte verwenden können. Auf der einen Seite steht der Anspruch auf vollständige Informationsaufnahme um ein umfassendes geographisches Bild zu kommunizieren, auf der anderen Seite das zufällige Durchstöbern der Karte, das als interessante und potentiell effektive Form des Informationsaustausches gilt. Cartwright fordert demzufolge eine Methode, die diese Ge- 
gensätze miteinander verbindet. Diese soll sicherstellen, dass die Nutzenden während der Bewegung durch die Karte alle wichtigen Informationen effizient sammeln können, jedoch gleichzeitig ein Maximum an Entdeckungsfreiheit innerhalb des Produkts genießen, um Inhalte weiterhin zu können.

Cartwright (2004) bringt in diesem Zusammenhang bei der Verwendung von geographischen Multimediaprodukten den Ansatz Engineered Serendipity vor. Diese Idee setzt sich aus zwei gegensätzlichen Begriffen zusammen: Serendipity beschreibt die Möglichkeit zu „happy and unexpected discoveries by accident" (Cartwright 2007, 7), einen Weg, zufällige Entdeckungen zu machen (siehe Abbildung 2-13). Wollen die Kartenentwickelnden trotzdem sichergehen, dass alle essentiellen Informationen wahrgenommen werden, kann serendipitous use dieses nicht sicherstellen. Die Kartenautoren greifen deshalb durch geplantes und konstruiertes Engineering ein. Engineered Serendipity wird eingesetzt um den Nutzenden eine scheinbare Freiheit beim Erkunden des Produkts zu geben, die gleichzeitig sicherstellt, dass keine essenziellen Informationen übersehen werden. Die Nutzenden können sich frei durch das System bewegen, bekommen allerdings eine Checkliste vorgelegt, bevor sie dieses wieder verlassen. Diese leitet die Nutzenden zu allen wichtigen Informationen hin, die eventuell noch nicht besucht wurden. Dieser Ansatz stellt sicher, dass die Nutzenden bei der Verwendung des Produkts die Möglichkeit haben, alle Informationen zumindest einmal gesehen haben (siehe Abbildung 2-14).

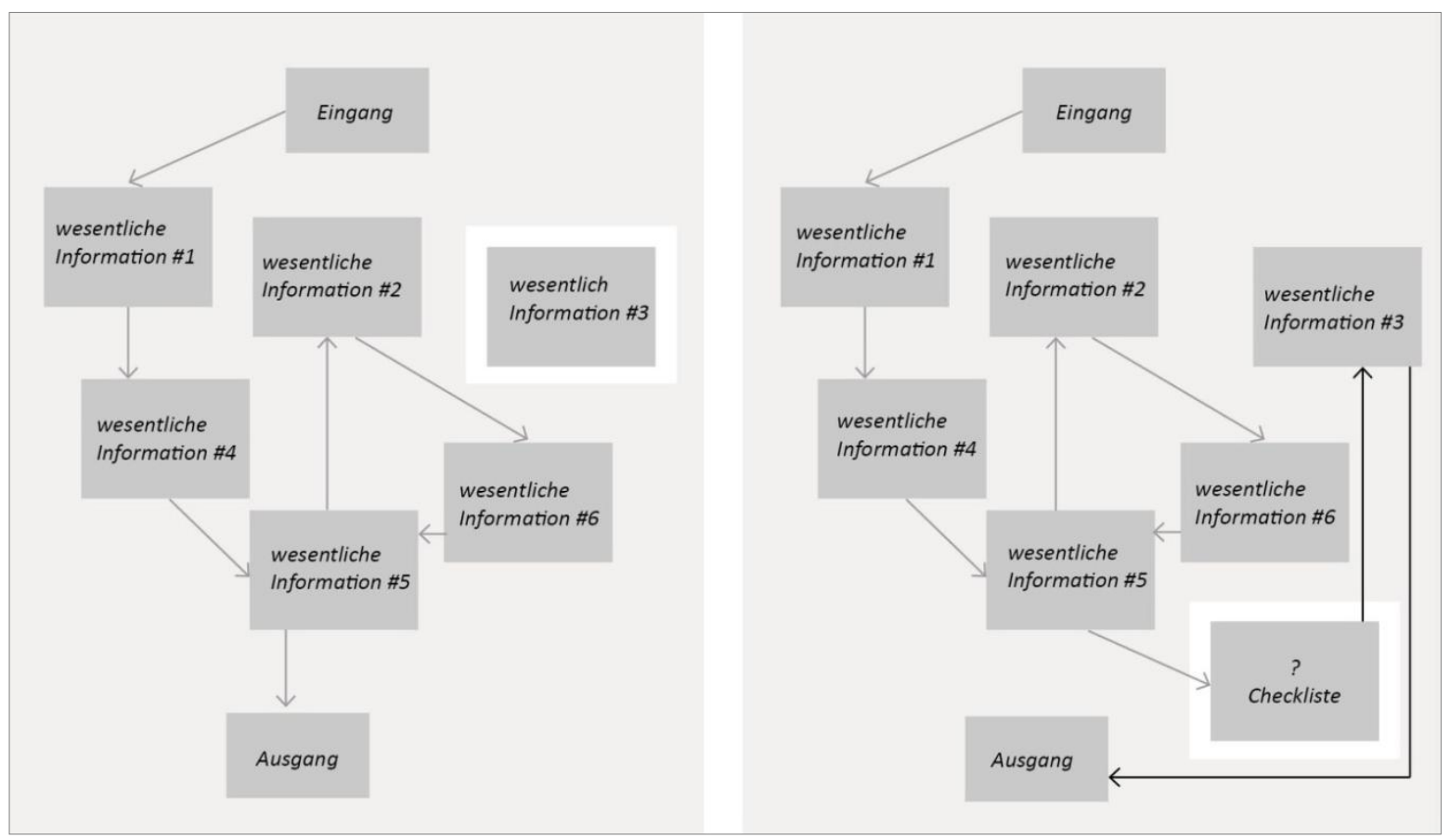

Abbildung 2-13 Der Serendipity-Ansatz führt zu zufälligen Entdeckungen (Cartwright 2004, 8)
Abbildung 2-14 Engineered Serendipity stellt sicher, dass alle Informationen zumindest einmal gesehen wurden (Cartwright 2004, 9)

\subsubsection{Erzähltechniken in Narrative Visualizations}

Segel und Heer (2010) untersuchen die Eigenschaften von Narrative Visualizations. Narrative Visualizations sind Visualisierungen von Daten, die den Nutzenden eine Geschichte erzählen sollen. Die Autoren unterscheiden anhand von verschiedenen Merkmalen unterschiedliche Gruppen von Narra- 
tive Visualizations. Als Merkmal zählt beispielsweise die Menge des Inhalts, die graphische Gestaltung, die gewählte Erzählstruktur, der Grad der Interaktivität und die Anzahl an erklärenden Begleittexten. Dabei identifizieren die Autoren zwei Hauptgruppen an Taktiken, wie die Data Story erzählt wird. Es gibt autorengesteuerte (author-driven) oder nutzergesteuerte (reader-driven experience) Methoden.

Zur autorengesteuerten Methode zählen Datenvisualisierungen, die in aufeinanderfolgenden Sequenzen strikt entlang eines linearen Pfades ablaufen. Diese Methoden beinhalten keine Interaktivität, sondern setzen ihren Schwerpunkt auf narrative Strukturen wie erklärende Texte, um die Visualisierung zu erläutern. In Comicstreifen oder Firmenpräsentationen kann diese Methode gefunden werden, da auch hier die Nutzenden keine Möglichkeit haben, in die präsentierten Inhalte einzugreifen. Nutzergesteuerte Methoden in Datenvisualisierungen befolgen hingegen keine vorgeschriebene Reihenfolge der präsentierten Darstellungen, sondern können interaktiv von den Nutzenden bedient werden. Die Interaktivität soll die Nutzenden dazu verleiten, sich intensiv mit der Darstellung auseinander zu setzen und auch eigene Fragestellungen und Hypothesen aus den präsentierten Daten abzuleiten. Tabelle 2-1 zeigt eine Übersicht der jeweiligen Charakteristiken.

\begin{tabular}{l|l}
\hline AUTORENGESTEUERTE METHODE & NUTZERGESTEUERTE METHODE \\
\hline $\begin{array}{l}\text { Linear aufeinanderfolgende Sequenzen } \\
\text { Erklärende Begleittexte ('heavy messaging') }\end{array}$ & Keine vorgegebene Reihenfolge der Sequenzen \\
\hline Keiner Interaktivität & Interaktivität \\
\hline
\end{tabular}

Tabelle 2-1 Eigenschaften von autoren- und nutzergesteuerten Methoden (Segel und Heer 2010, 8)

Segel und Heer (2010) weisen darauf hin, dass in der Praxis bei der Kommunikation von Informationen an die Nutzenden weder rein autorengesteuerte noch rein nutzergesteuerte Methoden verwendet werden. Vielmehr setzen sich die zu erzählenden Geschichten aus einer Mischung beider Methoden zusammen, was ein weiteres Spektrum zwischen diesen zwei Extremen entstehen lässt. Die Kombination dieser verschiedenen Elemente wird als eine der grundlegendsten Eigenschaften der Narrative Visualizations gesehen. Die dabei entstehenden Visualisierungstypen variieren aufgrund des Verhältnisses zwischen vorbestimmten Erzählfluss und selbstständigem Entdecken durch interaktives Explorieren. Verwendete graphische Elemente und das von den Autoren bestimmte Interface sind dabei bestimmend für die Möglichkeiten der Nutzersteuerung.

Als oft verwendete Mischformen werden von den Autoren folgende Narrative Structure Tactics (Taktiken, die bei der Kommunikation von Informationen angewendet werden) identifiziert:

\section{a) Martiniglas-Struktur}

Die Martiniglas-Struktur (siehe Abbildung 2-15) beschreibt eine Visualisierung, die aus zwei Abschnitten besteht. Der erste Abschnitt besteht aus einem verfassten Artikel oder aufgeworfenen Fragen, um autorengesteuert in die Visualisierung einzuleiten. Die Autoren können somit sicherstellen, dass 
ein bestimmtes erzählendes Element den Nutzenden präsentiert wird. Der autorengesteuerte Abschnitt dient als Ausgangpunkt für den sich anschließend öffnenden zweiten Abschnitt. In diesem wird die Visualisierung in einer nutzerorientierten Form präsentiert, in der die Nutzenden durch Interaktivität die Daten frei erkunden können. Anreize für ihre Exploration finden sie in den im ersten Abschnitt aufgeworfenen Themen (Segel und Heer 2010).

Namensgebend ähnelt diese Struktur der Gestalt eines Martiniglases. Der Stängel des Glases repräsentiert die autorengesteuerte Erzählung, das Glas selbst verkörperte die verschiedenen Pfade, die die Nutzenden durch die Interaktivität wählen können. Segel und Heer siedeln diese Methode aufgrund der starken Steuerung der Nutzenden im ersten Abschnitt der Visualisierung eher im Bereich der autorengesteuerten Methoden an (Segel und Heer 2010).

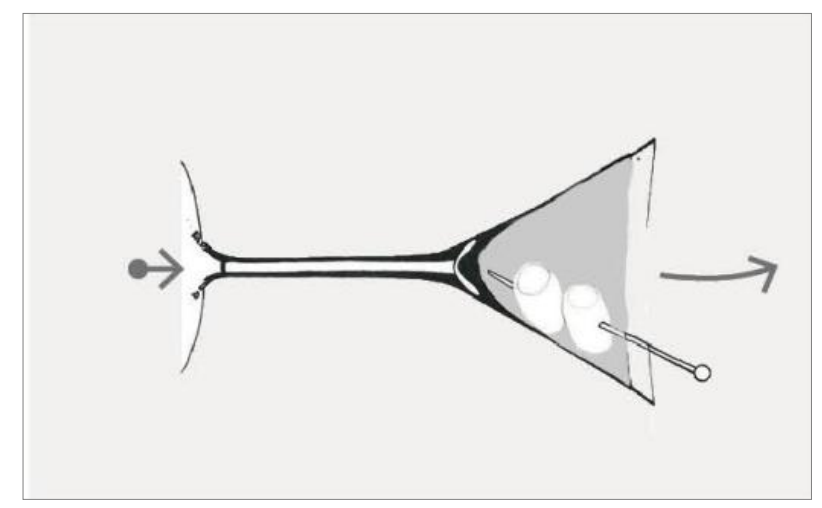

Abbildung 2-15

Die Martiniglas-Struktur als autorengesteuerte Methode (Segel und Heer 2010, 8)

\section{b) Interaktive Slideshow}

Diese Form der Narrative Visualization folgt der Struktur einer Diashow (Slideshow) (siehe Abbildung 2-16), bei der Inhalte in aufeinanderfolgender Weise präsentiert werden. Durch die Slideshow können besondere Inhalte der Präsentation von den Nutzenden detailliert erkundet werden, bevor zum nächsten Teil der Geschichte vorangeschritten wird (Segel und Heer 2010). Interaktive Slideshows eignen sich sowohl um komplexen Datensätze darzustellen, als auch um Erzählungen zu übermitteln. Bei komplexen Datensätzen können die Nutzenden Schritt für Schritt durch die Datenmengen geführt werden und gegebenenfalls einzelne Schritte wiederholt werden. Dadurch soll sichergestellt werden, dass die Nutzenden erst dann in der Präsentation weitergehen, wenn diese auch dafür bereit sind. Für die Vermittlung von komplexen Erzählungen können durch die Methode der Interaktiven Slideshow einzelne Teile der Geschichte von den Autoren voneinander abgetrennt werden. Diese Teilungen erfüllen den gleichen Zweck wie beispielsweise einzelne Szenen in einem Film, die das Verständnis der Zuschauer erhöht (Segel und Heer 2010).

Segel und Heer (2010) befinden die Interaktive Slideshow, im Gegensatz zur Martiniglas-Struktur, als eine zwischen autorengesteuerter und nutzergesteuerter ausgeglichene Methode. Bei der Darstellung der einzelnen Abschnitte (Slides), kann sich allerdings wieder der Martiniglas-Methode bedient werden. 


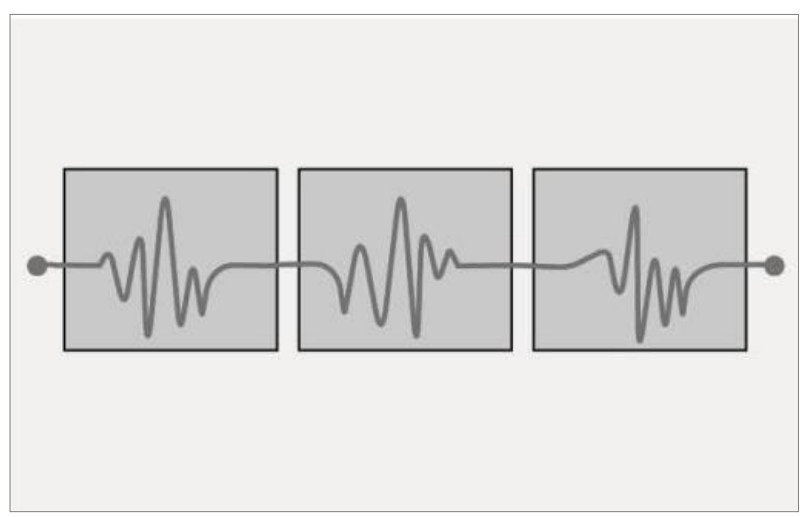

Abbildung 2-16 Die Interaktive Slideshow weist sowohl Eigenschaften von autoren- als auch nutzergesteuerter Methoden auf

(Segel und Heer 2010, 8)

\section{c) Drill-Down Story}

Die Drill-Down Story (siehe Abbildung 2-17) bedient sich einer Methode, bei der man sich von Überblicksinformationen durch hierarchisches Fokussieren zu Detailinformationen hinbewegt. In der Darstellung wird zuerst ein allgemeines Thema visualisiert, von dem aus die Nutzenden jene relevanten Bereiche auswählen können, von denen sie mehr und detailliertere Informationen sehen wollen (Segel und Heer 2010).

Segel und Heer (2010) sehen den Schwerpunkt dieser Visualisierungsmethode im nutzergesteuerten Zugang. Die Nutzenden können zu jederzeit bestimmen, welche Geschichte wann erzählt werden sollen. Dennoch verlangt dieser Zugang ein gewisses Maß an Steuerung durch die Autoren der Darstellung. Sie bestimmen die Möglichkeiten der Interaktion zwischen Nutzenden und Karte, ebenso legen sie die zur Auswahl stehenden Geschichten und ihre Details fest.

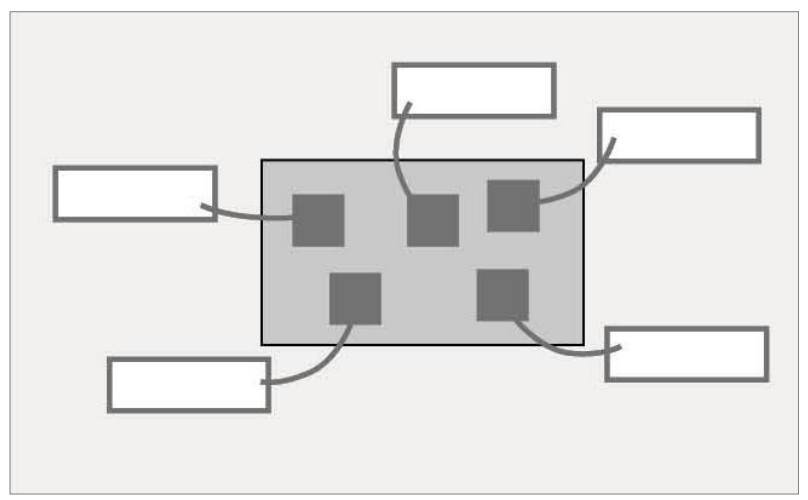

\section{Abbildung 2-17}

Die Drill Down Story als nutzergesteuerter Zugang (Segel und Heer 2010, 8)

Für Segel und Heer (2010) erschließt sich, dass Visualisierungen die mit Kontrollpunkten innerhalb der Erzählung ausgestattet sind, die effektive Form von Data Stories sind. Es bleibt den Nutzenden weiter erlaubt zu explorieren, wobei jedoch sichergestellt wird, dass sich diese nicht zu weit von der beabsichtigen Erzählung entfernen.

\subsubsection{Zusammenfassung}

Die in Kapitel 2.4.1 behandelten Möglichkeiten stellen eine Auswahl an der in der Literatur angeführten Methoden zur Benutzerführung in kartographischen Produkten dar. 
Obwohl durch die Verbreitung von höchst dynamischen und interaktiven Kartenprodukten heutzutage Konzepte zur Nutzerführung besonders aktuell sind, gab es schon vor mehr als 20 Jahren Diskussionen über Nutzersteuerung in geographischen Informationsprodukten. Cartwright, Monmonier, sowie Segel und Heer beschreiben unterschiedliche Herangehensweisen aus unterschiedlichen Kontexten heraus, die mit dem Erscheinungszeitpunkt und den dementsprechenden technischen Entwicklungen der geographischen Produkte zusammenhängen. Dennoch ist festzustellen, dass sich die Ansätze in ihrer grundsätzlichen Aussage stark ähneln. Die Konzepte vorfolgen alle dasselbe Ziel, nämlich sicherzustellen, dass die Informationen, die von den Kartenautoren kommuniziert werden soll, von den Nutzenden auch wahrgenommen wird. Als starkes Merkmal, dass sich durch viele der oben angeführten Konzepte zieht, ist die Begrenzung der Freiheit auf der Seite der Nutzenden. In vielen Fällen verfügen diese nicht über die Freiheiten, die sie bei der Benützung vermeintlich wahrnehmen, da die Interaktionen der Nutzenden mit den Inhalten der Karte innerhalb von vorgegebenen Grenzen geschehen. Die Autoren bleiben dadurch die wahren Leiter bei der Verwendung des kartographischen Produkts. Die festen Vorgaben der Kartenautoren werden beispielsweise anhand der von Cartwright (1999) beschriebenen Storyteller-Metapher und seinem Ansatz Engineered Serendipity, sowie in der von Segel und Heer beschriebenen Interaktiven Slideshow verdeutlicht. In der Storyteller-Metapher werden die Nutzenden durch begrenzte Interaktionsmöglichkeiten beschränkt. Im Engineered Serendipity Ansatz werden die Nutzenden erst dann aus der Anwendung entlassen, wenn sie alle Inhalte aufgerufen haben. Die Interaktive Slideshow zeichnet sich dadurch aus, dass die Nutzenden eine strikte Vorgabe über die Reihung der präsentierten Inhalte haben. Auch das spezielle Auswählen und Hinführen zu Informationen, die für die Nutzenden als relevant eingestuft werden, tritt in den Metaphern Navigator und Guide auf. Der Navigator verweist dabei auf relevante Orte, die besucht werden sollen und der Guide fokussiert nur auf wichtige Informationen, indem er irrelevante ausspart.

Cartwright (2004) sowie Segel und Heer (2010) sind sich einig, dass um eine optimale Informationsaufnahme für die Nutzenden zu bieten, eine gewisse Steuerung durch die Kartenautoren mit begrenzter Interaktivität einer völlig freien Bewegung vorzuziehen ist. Wie sich diese Steuerung jedoch auf die Benützung von Karten auswirken kann, soll anhand der im folgenden Punkt beschriebenen Hypothesen dargelegt werden.

\subsubsection{Interpretationshilfe und Hypothese zu Nutzerführungen in Karten}

Auch die Interpretationshilfe in KIT-Karten ist im Kontext zur Nutzerführung einzuordnen. Die Interpretationshilfe versucht, ebenso wie die oben dargelegten Konzepte, sicherzustellen, dass alle relevanten Informationen von den Nutzenden wahrgenommen werden. Erklärende Texte führen die Nutzenden durch Visualisierungen geographischer Daten. Die erklärenden Texte der Interpretationshilfe sollen sicherstellen, dass bestimmte räumliche Zusammenhänge von den Nutzenden auf jeden Fall erkannt werden und die von Kartenautoren vorgesehene Aussage der Karte verstanden wird.

Die Interpretationshilfe weist einige Ähnlichkeiten mit den oben ausgeführten Konzepten auf. Eine der größten Gemeinsamkeiten besteht mit dem Engineered Serendipity Ansatz und der autorengesteuerten Erzähltaktik der Narrative Visualizations. Wie bei Engineered Serendipity wird den Kartenautoren durch die Interpretationshilfe die Möglichkeit gegeben, durch textliche Unterstützung sicherzustellen, dass bestimmte Informationen auf jeden Fall den Nutzenden präsentiert werden. Die Nutzenden können weiterhin eigene Entdeckungen innerhalb der Karte machen, kommen allerdings 
an der Präsentation bestimmter Informationen nicht vorbei. In Hinsicht auf die Kategorisierung der Narrative Visualizations, steht die Interpretationshilfe im Zusammenhang mit autorengesteuerten Data Stories. Die Aufgabe der Interpretationshilfe kann als eine der beschreibenden Eigenschaften von autorengesteuerten Methoden gesehen werden, da sie erklärende Kommentare für die dargestellte Visualisierung bereitstellt.

Weiters lassen sich Gemeinsamkeiten zwischen Interpretationshilfe und den von Cartwright (1999) beschriebenen Metaphern feststellen. Die Navigator und Guide Metapher verfolgen ähnliche Ziele wie die Interpretationshilfe, indem sie auf Bereiche mit wichtigen Informationen hinweisen und relevante Informationen für die Nutzenden herausheben. Auch Charakteristiken der Graphic Scripts finden sich in der Interpretationshilfe wieder. Die Gestaltung der Bewegung der Nutzenden durch die Karte wird durch die Interpretationshilfe ähnlich diesem Ansatz. Es entsteht allerdings bei dem Einsatz der Interpretationshilfe keine Animation durch die Zusammensetzung mehrerer Karten, jedoch werden die Kartennutzenden wie bei den Graphic Scripts anhand eines vorgegebenen Pfades durch die Anwendung geführt.

Wie bereits als ein Merkmal der beschriebenen Nutzerführungskonzepten identifiziert wurde, setzt auch die Interpretationshilfe auf eine geplante Führung der Nutzenden, die vor das völlig freie Explorieren im Kartenprodukt gestellt wird. KIT-Karten mit Interpretationshilfen können daher als autorengesteuerte Methode zur Kommunikation von Informationen an Nutzende gesehen werden. Wie in Kapitel 2.4.1.4 beschrieben, nennen Segel und Heer (2010) als eine der wichtigen Charakteristiken nutzergesteuerter Erzähltaktiken den Anreiz an die Nutzenden, durch Explorieren eigene Gedanken zu den visualisierten geographischen Daten zu entwickeln. Diese Anregung kann durch Implementierung der Interpretationshilfe in KIT-Karten in den Hintergrund gedrängt werden. Wie bereits in Kapitel 2.4.1.4 a in Bezug auf die Martiniglas-Taktik erwähnt wurde, birgt der Gebrauch von erläuternden Texten die Gefahr, Nutzende in ihrem Explorationsverhalten zu beeinflussen. Durch die starke inhaltliche Beschreibung, die durch die Interpretationshilfe geboten wird, können die Nutzenden mit einer voreingenommenen Haltung an die Interpretation der Visualisierung heran gehen. KIT-Karten können als Resultat nicht mehr selbstständig und explorativ nach Zusammenhängen von Sachverhalten erkundet werden, da sich die Nutzenden zu stark auf die Aussage der Interpretationshilfe stützen könnten. Durch folgende Hypothese soll dieses Thema im Zuge des Usability-Tests geprüft werden:

Hypothese 1 Die Verwendung einer Interpretationshilfe führt zur Interpretation von bestimmten Inhalten. (siehe Kapitel 2.3.6)

Hypothese 3 Die Verwendung einer Interpretationshilfe führt zu einem verminderten Explorieren in der Karte.

\subsubsection{HILFESYSTEME IN SOFTWAREPRODUKTEN}

Nachdem in Kapitel 2.4.1 Herangehensweisen zur Benutzerführung in Produkten mit kartographischem Bezug vorgestellt wurden, beschäftigt sich dieser Abschnitt mit Unterstützung in diversen Softwareprodukten. Viele der folgenden Zugänge finden ihre Anwendung hauptsächlich in technischer und systembezogener Unterstützung von Anwendungen. Es liegt jedoch nicht fern, diese Me- 
thoden auch unter dem Aspekt von inhaltsbezogener, thematischer Unterstützung zu betrachten, die bei Verwendung einer Interpretationshilfe relevant werden.

Dieses Kapitel widmet sich einer Übersicht über die üblich umgesetzten Hilfesysteme und beschreibt Möglichkeiten zur Implementierung. Weiters werden auch ihre Kritikpunkte angeführt, die in Verbindung mit der Umsetzung der Interpretationshilfe gesetzt werden.

\subsubsection{Hilfesysteme und Online-Hilfe}

Allgemein versteht man unter Hilfesystem ein oder mehr Programme, die im Zusammenhang mit einem größeren Programm oder Computersystem den Nutzenden Unterstützung bieten. Hilfesysteme werden vor allem in jenen Bereichen des Systems eingesetzt, mit deren Verwendung die Nutzenden nicht vertraut sind. Die Hilfesysteme unterstützen die Nutzenden bei jenem Lernprozess, der konzeptuelle oder mentale Modelle entwickelt, die das Verhalten des Systems erklären (Galitz 2007).

Als häufig eingesetzte Form eines Hilfesystems kommt die Online-Hilfe (Online Help System) zum Einsatz. Sie umfasst jede Art von Kommunikation, die online bereit gestellt wird, um die Nutzenden bei einer effektiven Arbeitsbewältigung behilflich zu sein (Galitz 2007). Üblicherweise wird das Online-Hilfesystem als Sammlung kurzer, aufgabenorientierter Informationselemente definiert, die die Nutzenden bei der Erfüllung ihrer Aufgaben unterstützen (Corbin 2003). Duffy et al. definieren die Online-Hilfe als leistungsorientierte Informationen, die online präsentiert werden und die Frage nach dem „Wie mache ich das?" beantworten. Es geht im Gegensatz zu Online-Tutorials oder Lehrmaterial nicht darum etwas zu lernen, sondern um eine bestimmte Aufgabe in einer Anwendung auszuführen. Die Nutzenden werden dafür mit Informationen versorgt, die entweder als Fakten, Abläufe oder Erklärungen präsentiert werden. Online-Hilfen werden in fast allen Softwarepaketen bereitgestellt und finden sich beispielsweise in Microsoft Office Anwendungen oder Computerspielen. Sie zeichnen sich dadurch aus, dass sie leicht aufgerufen werden können und unmittelbare, informative und bildschirmorientierte Durchführungsunterstützung bieten (Hall 2006). Allgemein besteht die OnlineHilfe, so wie jede andere Art von Hilfesystemen, einerseits aus dem Interface, das festlegt wie die Hilfe aufgerufen und dargestellt wird und andererseits aus dem Inhalt, der die Informationen der Hilfe enthält. Damit ein Hilfesystem zweckerfüllend ist, ist auf beide Aspekte Bedacht zu nehmen. Es muss demzufolge sowohl gut zu verwenden als auch inhaltlich verständlich sein (Kearsley 1988).

Dieses Kapitel setzt sich nicht mit dem enthaltenen Inhalt der Hilfe auseinander, sondern legt den Schwerpunkt auf die Einbettung der Hilfe in das Interface der Anwendung, das den Nutzenden Zugang zu den Hilfeinformationen ermöglicht. Je nach funktionaler Charakteristik der Online-Hilfe kann sie verschieden klassifiziert werden. Galitz (2007) unternimmt folgende Einteilung von Online-Hilfen.

\section{a) Kontextabhängige Hilfe}

Die kontextabhängige Hilfe (Contextual Help, Context-Sensitive Online Help System) stellt Informationen für den aktuellen Kontext der durchzuführenden Aufgabe oder über das spezifische Objekt mit dem gearbeitet wird, dar (Galitz 2007). Die Hilfeinformationen werden durch kleine Stücke in Interfaceobjekte eingebettet. Üblicherweise handelt es sich dabei um Information, die sich meist ausschließlich auf primitive Operationen im Kontext der Computeranwendung beziehen und wenig auf die Hauptaufgabe, die die Nutzenden eigentlich durchführen möchten, reagiert (Ye 2001). Die kon- 
textabhängige Hilfe kann beispielsweise durch Tooltips oder Berater Informationen an die Nutzenden bringen.

\section{b) Aufgabenorientierte Hilfe}

Die aufgabenorientierte Hilfe (Tast-Oriented Help) versucht, den Schwächen der kontextabhängigen Hilfe zu begegnen, indem sie die angebotene Information besser auf die auszufüllende Aufgabe abstimmt. Die Information wird dazu im Hauptfenster präsentiert, in dem alle nötigen Arbeitsschritte zur Aufgabeerfüllung detailliertet aufgelistet werden. Diese Art von Unterstützung wird von den Nutzenden meist nicht nur gegenüber produktorientierter, kontextabhängiger Hilfe bevorzugt, sondern auch Forschungsergebnisse belegen die höhere Produktivität bei Gebrauch dieser Hilfe. Sie schlägt ihren Vorteil daraus, dass Menschen in Aufgaben und nicht in Funktionen denken. Hinweise zu Wie etwas gemacht wird, ist für Nutzende meist hilfreicher als Informationen zu Was und Warum der produktorientierten Hilfe (Galitz 2007). On-Screen Instructions und Wizards sind Beispiele für aufgabenorientierte Hilfen.

\section{c) Referenzorientierter Hilfe}

Bei referenzorientierter Hilfe (Reference Help) werden die Informationen über Dokumentationen oder über das Benutzerhandbuch präsentiert. Typischerweise wird sie über eine Schaltfläche im Hilfe-Dropdown-Menü oder über die Werkzeugleiste aufgerufen. Die Darstellung der Information wird meist über die Funktionen der Anwendung organisiert und enthält mehr Text als in anderen Hilfesysteme (Galitz 2007). Aufbau und Organisation der referenzorientierten Hilfe entsprechen weitgehend einer traditionellen Aufbereitung der Online-Hilfe. Durch eine Serie von Themen und Unterthemen werden die Inhalte des Produkts präsentiert, die über Menüs, Inhaltsverzeichnisse, Index Suchfunktionen und Hypertext aufgerufen werden können (Hall 2006; Keyes 2002).

\subsubsection{Implementierungsmöglichkeiten von Online-Hilfen}

Hilfesysteme können entweder direkt in das Anwendungssystem integriert oder separat und gleichzeitig mit dem System laufen. Kontextabhängige Hilfe, aufgabenorientierte Hilfe oder referenzorientierte Hilfe unterscheiden sich auch in ihrer Implementierung in die Anwendung. Referenzorientierte Hilfe wird durch einen separaten Zugriff auf traditionelle Weise eingebettet, kontextabhängige und aufgabenorientierte Hilfe werden hingegen direkt in die Anwendung integriert.

Hall (2006) beschreibt Schwierigkeiten, die bei traditionaler Einbettung von Hilfesystemen in Anwendungen auftreten können. Beispielsweise ist die Navigation nicht immer so gestaltet, dass relevante Elemente leicht gefunden werden können. Weiters wird oft ein Übermaß an Informationen geboten, die nicht hilfreich sind, da die verwendete Terminologie schwer verständlich ist. Ist die Hilfe nicht ausreichend inhaltsspezifisch angepasst und eindeutig aufzurufen, kann sie für die Nutzenden eine Behinderung bei der Erfüllung ihrer Aufgaben sein.

Um der Problematik traditioneller Einbettung zu entgegnen, gilt als derzeit vorherrschendes Design zur Umsetzung von Online-Hilfen das integrierte Hilfesystem (Embedded Assistance/Help). Es ermöglicht es beispielsweise Tooltips, Wizards, Assistenten und eingebettete Hilfefenster in Programme einzubinden. Im Gegensatz zu anderen Hilfesystemen, wo Nutzende explizit nach einer zusätzlichen Assistenz durch ein peripheres Unterstützungssystem fragen müssen, werden die Informationen nutzerorientiert als integraler Teil der Anwendung direkt in das Interface eingebettet. Texte und An- 
leitungen in den Bildansichten führen die Nutzenden und assistieren bei der Durchführung von Aufgaben. Da keine aktiven Handlungen und Initiativen der Nutzenden notwendig sind, werden die Nutzenden bei ihrer Arbeit wenig gestört (Hall 2006; Keyes 2002; Corbin 2003).

Im Folgenden werden nun einige Umsetzungsvarianten von integrierten Hilfesystemen näher vorgestellt.

\section{a) Hinweise und Tipps}

Informationen, die durch Hinweise und Tipps dargestellt werden, zeichnen sich durch ihre hohe Relevanz für die momentane Situation aus, da sie wichtige und spezifische kontextbezogene Informationen enthalten. Die Hilfe ist für die Nutzenden leicht zu erreichen und leistet rasche Unterstützung bei unklaren Anforderungen (Galitz 2007).

Hinweise und Tipps können Informationen in unterschiedlicher Form in die Anwendung integriert werden. Die Hilfe kann beispielsweise in einem zusätzlichen Hilfefenster einblendet werden, auch Befehlsfragen an die Nutzenden sind möglich, um Tipps zur Durchführung der Aufgabe bereitzustellen (Alliance for Technology Access 2004). Weiters können Informationen durch Meldung in der Statusleiste des Hauptfensters angezeigt werden, wenn der Mauszeiger über ein bestimmtes Objekt bewegt wird. Auch Hilfeblasen, sogenannten Tooltips oder Balloon Help, werden verwendet, um die Nutzenden mit Informationen zu unterstützen. Wie in Abbildung 2-18 gezeigt, werden bei dieser Art von Unterstützung Hilfetexte mittels kleinen Pop-Up-Fenstern neben dem Mauszeiger im Vordergrund anzeigt (Galitz 2007; Hall 2006). Beim Anzeigen von Hinweisen und Tipps haben die Nutzenden nicht immer selbst die Möglichkeit über die Anzeigedauer der Informationen auf dem Bildschirm zu entscheiden (Alliance for Technology Access 2004; Galitz 2007).

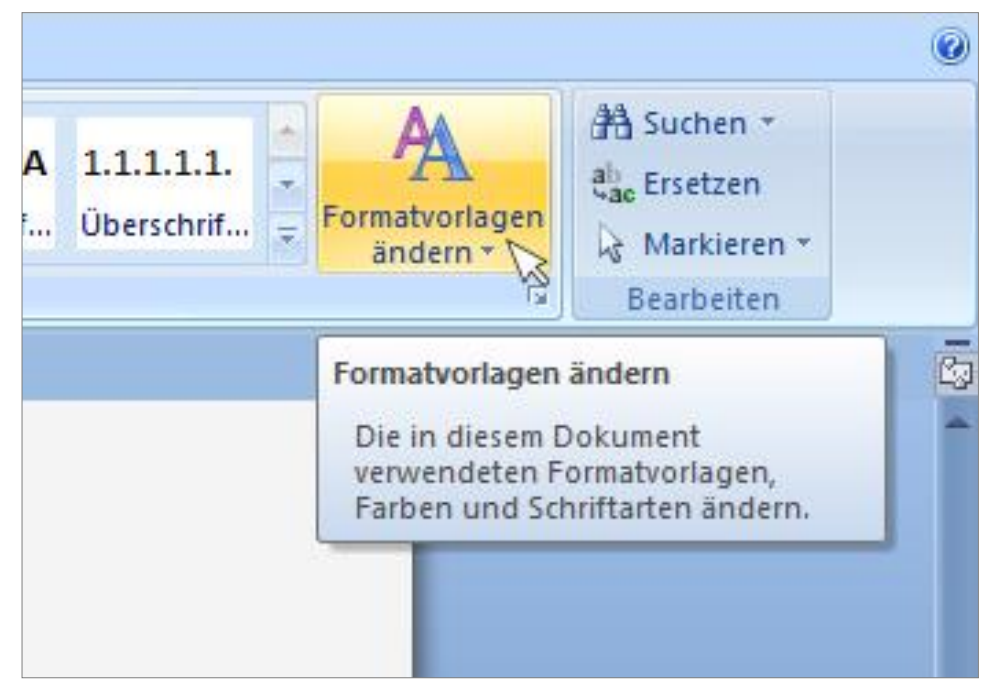

Abbildung 2-18

Tooltip in Microsoft Office Word 2007 bei aktiver Maus

(Screenshot)

\section{b) Persönliche Assistenten}

Berater, Agenten oder Active Advices (aktive Hinweise) sind eine weitere Variante zur Umsetzung von kontextbezogenen Hilfesystemen. Die Initiative zur Unterstützung der Nutzenden übernimmt das System, das Wissen präsentiert, welches den Nutzenden zu fehlen scheint. Das System holt Informa- 
tionen ein, kontrolliert automatische Prozesse um aufgabenrelevante Erklärungen und Antworten auf Probleme der Nutzenden zu geben. Hilfestellung durch Assistenten kann weiters dafür herangezogen werden, die Nutzende durch interaktive Demonstrationen bei der Aufgabenbewältigung zu unterstützen und bei der Navigation in komplexen Umgebungen zu helfen (Laufmann 1998).

Die Implementierung geschieht meist durch persönliche Assistenten, der die Aktionen der Nutzenden beobachtet. Just-in-Time-Hilfen, die während der Ausführung des Programms angezeigt wird, bieten Unterstützung zum Wissensgebiet oder auch zur Anwendung selbst und schlagen bessere Möglichkeiten zur Aufgabeerfüllung vor. Meist werden diese Berater als animierte Menschen oder Tiere dargestellt, die durch menschliches Verhalten versuchen, die Aufmerksamkeit der Nutzenden zu gewinnen, um mit ihnen zu interagieren (Hall 2006; Laufmann 1998).

Eines der Bekanntesten Beispiele dieser Hilfe ist der von Microsoft Entwickelte Assistent Clippy, der in einer bereits längst überholten Version von Word eingesetzt wurde. Abbildung 2-19 zeigt durch welche Charaktere der Assistenten in das Programm implementiert wurde. Sobald in dem Programm eine Wohnadresse eintippt wurde, auf die ein "Liebe" oder "Lieber" folgte, tauchte Clippy mit einem vorgefertigten Set an Hinweisen zum Schreiben von Briefen auf.

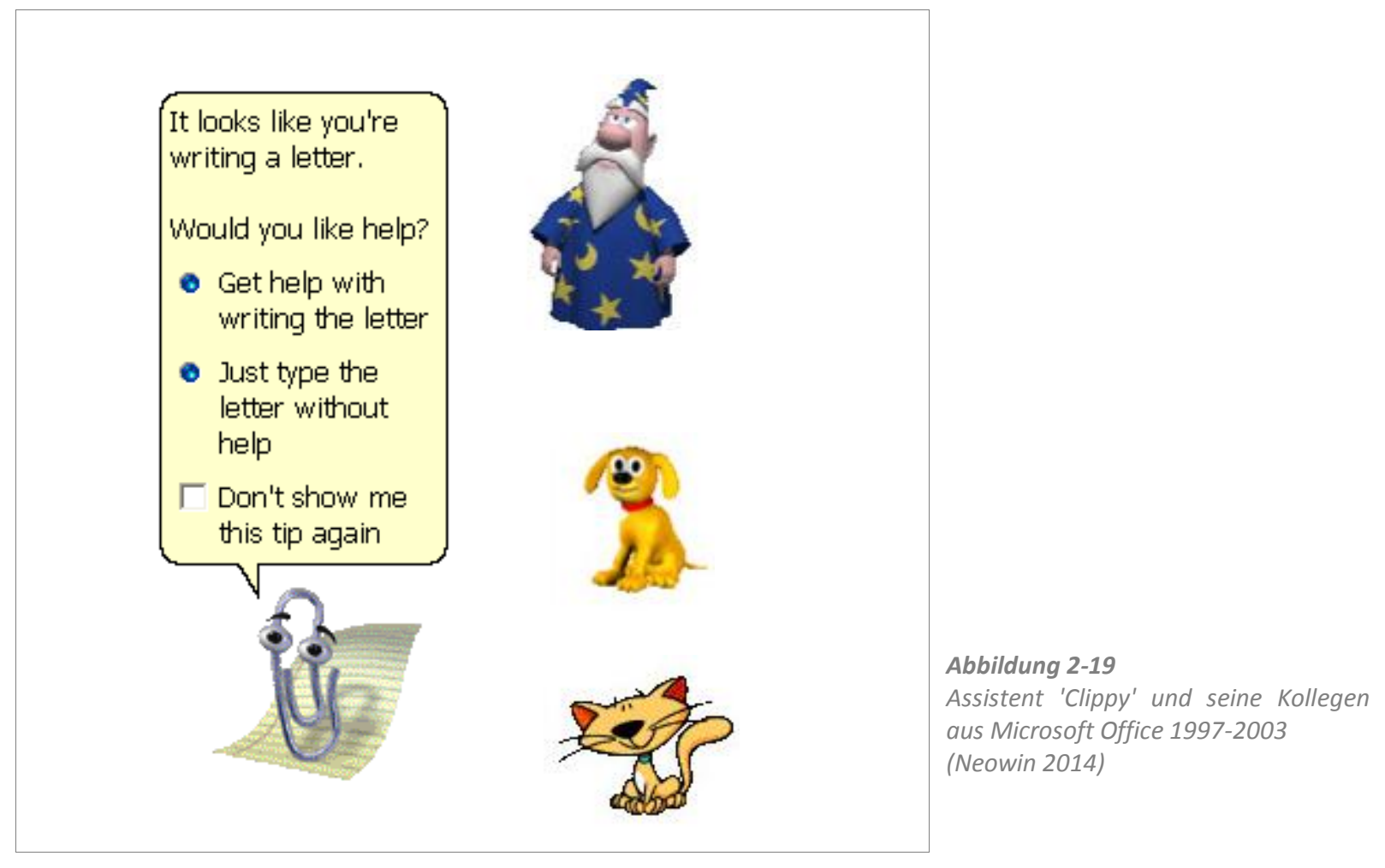

Ein zukunftsträchtiger Bereich im Einsatz von Beratern ist der sprachgesteuerte Assistent. Seine Aufgabe liegt neben Spracherkennung zur Erleichterung der Bedienbarkeit besonders darin, die Nutzenden bei kognitiver Überbelastung (congnitive overload) zu unterstützten. Die virtuellen Assistenten basieren auf einer Software, die vor allem den Kontext in dem die Nutzenden Unterstützung suchen, berücksichtigen. In diesem Zusammenhang wird auch der Begriff der 'künstliche Intelligenz' verwendet, da die Funktion der Assistenten sehr stark darauf ausgerichtet sind, die Nutzenden und ihre Bedürfnisse zu verstehen (Naone 2009; Gouin et al. 2012).

Ein derzeit sehr populäres Beispiel eines virtuellen Assistenten ist Siri. Siri ist ein Softwareprodukt der Firma Apple, das in iPhones eingesetzt wird, um geschriebene oder gesprochene Aufgaben entgegen- 
zunehmen. Wird beispielsweise nach Unterstützung bei der Auswahl einer Wochenendaktivität gefordert, kombiniert Siri diese Anfrage mit dem derzeitigen Standort der Nutzenden, seinem Terminkalender, seinen bisherigen Aktivitäten, dem aktuellen Wetterbericht als auch mit den anstehenden Veranstaltungen. Diese Funktion ermöglicht es, den Nutzenden eine kontextabhängige Unterstützung zu bieten (Naone 2009).

\section{c) On-Screen Instructions}

On-Screen Instructions sind Dialoge und Anleitungen, die am Bildschirm angezeigt werden, um beim Lösen einer Aufgabe behilflich zu sein (siehe Abbildung 2-20). Die Anleitungen stehen im direkten Zusammenhang mit den Handlungen der Nutzenden, sodass die Nutzenden diese nicht umgehen können, sondern zur interaktiven Hilfe verpflichtet werden. On-Screen Instructions sind hinsichtlich ihrer proaktiven und strukturierten Charakteristik gut geeignet um prozessuale Tätigkeiten zu unterstützen. Sie werden sowohl als Hilfe zur Orientierung in Systemen eingesetzt oder auch um spezifische Hinweise zu Wissensgebieten zu geben. Aufgrund ihrer Eigenschaften zählen die On-Screen Instructions zu den wichtigsten Formen von integrierten Hilfesystemen. Besonders bedeutend sind sie für diverse Aufgabenlösung im Internet. Wenn nicht erwartet wird, dass Nutzende aktiv nach Hilfe suchen, werden integrierte Hilfesysteme als vorherrschende Unterstützung eingesetzt. So bedienen sich beispielsweise viele Bezahlungs- und Buchungssystemen im Internet Anleitungen, die im Dialog angezeigt werden (Hall 2006).

\section{Passagierinformationen}

Bitte geben Sie die Passagiernamen genauso ein, wie sie auf dem ausgestellten Lichtbildausweis erscheinen. Die i Nein. Die Fluggesellschaften und die Einreisebehörden erlauben keine Varianz beim Passagier-Namen; der Nachname muss komplett ausgeschrieben werden.

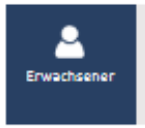

\begin{tabular}{|l|l|l|}
\hline Anrede: Norname: & Zweiter Vorname: Nachname: \\
\hline Auswählent &
\end{tabular}

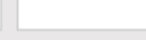

Tag $\boldsymbol{\nabla}$ Monat $\mathbf{V}$ Jahr

Open Advanced Preferences

\section{Preiszusammensetzung}

Geben Sie hier Ihren Gutscheincode ein, um von einem Rabatt zu profitieren

\section{Zahlungsoptionen}

- Bitte beachten Sie, dass die Passagier- und Zahlungsdaten mit lateinischen Buchstaben geschrieben werden sollten und keine griechischen Buchstaben, Umlaute oder Sonderzeichen enthalten (wie z.B.: ., ; : @ !)

\section{$\mathbf{2}$ \\ Kontaktdaten}

Kreditkartenzahlung

Abbildung 2-20 Dialog in einem Online-Buchungssystem für Flugreisen (AirFastTickets 2014) 


\section{d) Wizards}

Wizards sind interaktive Hilfswerkzeuge, die Nutzende bei langwierigen und mühsamen Aufgaben unterstützen. Sie werden bei komplexen oder selten durchgeführten Aufgaben eingesetzt, bei denen Nutzende Schwierigkeiten haben, diese zu erlernen oder auszuführen. Ein Wizard besteht aus einem strukturierten Set von Fenstern, das den Nutzenden durch komplexe Aufgaben führt. Dabei kann es sich beispielsweise um Entscheidungs- oder Dateneintragungsprozesse wie Softwareinstallierung, Erstellen eines Dokumenttyps oder Konfiguration eines Systems handeln. Der Wizard ermöglicht es, notwendige Prozesse zu automatisieren und in viele Schritte aufzuteilen. Er durchschreitet diese mit den Nutzenden, die von der dahinterstehenden Komplexität der Durchführung nichts mitbekommen (Galitz 2007; Silver 2004). Wizards werden üblicherweise über die Werkzeugleiste oder ein entsprechendes Symbol aufgerufen und in einem untergeordneten Fenster angezeigt. Diese enthalten Bedienungselemente zum Sammeln von Eingaben und Befehlsknöpfe zur Navigation. Die Schritte der Aufgaben werden nacheinander präsentiert und müssen vom Nutzenden ausgeführt werden, um zum Nächsten zu gelangen. Die Bewegungsfreiheit des Nutzenden ist meistens auf Vorwärts- und Rückwärtsbewegung eingeschränkt (Galitz 2007; Silver 2004). Abbildung 2-21 zeigt ein Beispiel eines Wizards zur Installierung einer Softwareanwendung.
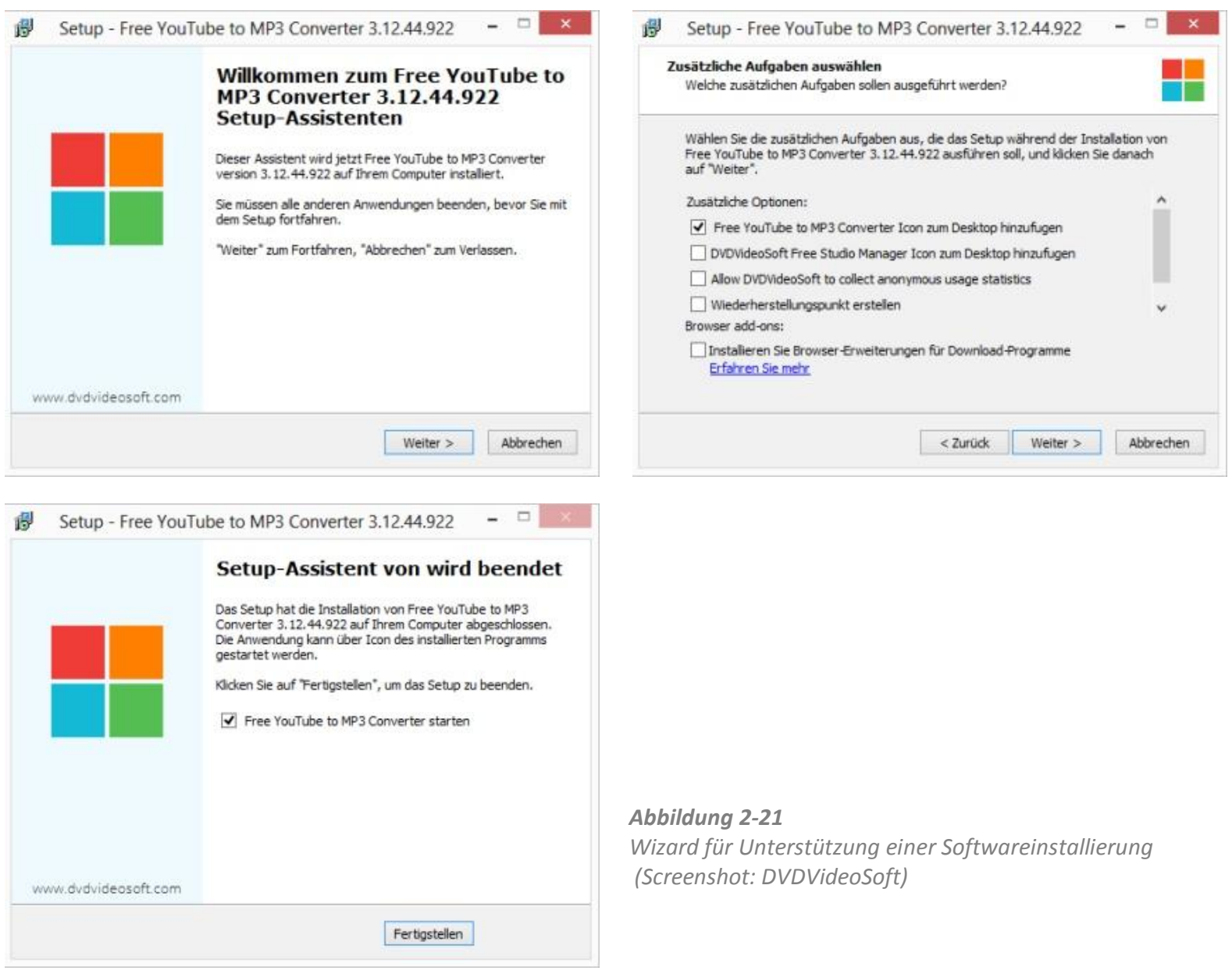

Abbildung 2-21

Wizard für Unterstützung einer Softwareinstallierung (Screenshot: DVDVideoSoft)

\subsubsection{Kritik an Hilfesystemen}

Neben den Vorteilen, die unterschiedlichen Hilfesysteme bieten, äußern einige Autoren auch Bedenken bei ihrer Umsetzung und Anwendung. Corbin (2003) bezieht sich auf verschiedene Studien, die 
die Zweckmäßigkeit von Hilfesystemen untersuchen. Zum einen wurde die Nützlichkeit von Hilfesystemen bei der Durchführung von Aufgaben bestätigt, zum anderen gibt es auch Studien, die das Gegenteil belegen. Diese zeigen auf, dass Hilfestellungen zu einer Beeinträchtigung bei der Aufgabendurchführung führen können, wenn diese nicht aufgabenorientiert umgesetzt sind und die Benutzeroberfläche komplexer machen.

Hall (2006) kritisiert weiters die in Online-Hilfen verwendete Terminologie. Er weist darauf hin, dass Hilfesysteme von Nutzenden, die sich erst kurz mit der Anwendung beschäftigen, nicht gut angenommen werden. Oft könnten sie aus dem Grund gemieden werden, da von den Nutzenden angenommen wird, dass die angebotene Unterstützung wegen der verwendeten Terminologie, ohnehin nicht verstanden wird.

Weiters beschreibt Hall (2006) in seiner Arbeit, dass auch der Einsatz von persönlichen Assistenten aufgrund ihrer penetranten Erscheinung kritisch betrachtet werden kann. Er warnt davor, dass diese schnell überanimiert sein können und die Nutzenden vor allem in den unbenutzten Momenten von ihrer Tätigkeit ablenken können. Cooper et al. (2007) zweifeln weiters an der Intelligenz dieser animierten Hilfe und entlarven diese als "Scharlatane" (Cooper et al. 2007, 511), die ohne genügend Wissen die Nutzenden "wütend machen" (Cooper et al. 2007, 511), wenn diese lieber ungestört ihre Arbeit erledigen wollen. Als prominentes Beispiel erfreute sich hierbei Microsoft's Clippy großer Unbeliebtheit. Aufgrund seiner praxisfernen und aufdringlichen Art wurde Clippy im Jahr 2010 vom Time Magazine in die Liste der 50 schlechtesten Erfindungen gewählt.

Galitz (2007) erklärt am Beispiel des Wizards, dass Hilfesysteme keine Werkzeuge sein müssen, die dabei helfen, etwas zu verstehen oder zu lernen, wie etwas getan wird. Auch Silver (2004) führt an, dass der Einsatz von Wizards in erster Linie die Schnelligkeit der Durchführung gegenüber der Leichtigkeit des Verstehens einer Handlung unterstützt. Cooper et al. (2007) identifizieren den alleinigen Zweck von Wizards darin, den Erfolg einer Operation zu garantieren. Die Autoren meinen, dass durch ihren Einsatz die Nutzenden lernen, in jedem Dialogfeld nur den Weiter-Knopf zu bedienen, ohne kritisch zu analysieren warum. Erfahrene Nutzende, die mit den Abläufen in der Anwendung vertraut sind, können Wizards demnach als " ineffiziente Werkzeuge" (Galitz 2007, 567) empfinden und bemängeln, dass nicht zu allen Funktionen Zugang geschaffen wird. Weiters weist Silver (2004) darauf hin, dass ihre Verwendung von den Nutzenden als "frustrierend" (Silver 2004, 162) wahrgenommen werden kann.

Corbin (2003) zitiert Forschungsergebnisse, die darstellen, dass Nutzende Online-Hilfesysteme meiden, da ihre Gebrauch einiges an Zeit kostet, die nicht vergütet wird.

\subsubsection{Zusammenfassung}

Es gibt viele verschiedene Möglichkeiten wie Hilfen umgesetzt werden können. Je nachdem welchen Zweck sie erfüllen sollen, verfügen sie über eine unterschiedliche funktionelle Charakteristik. Zusammenfassend ist jedoch zu erkennen, dass sich bezogen auf die Weise nach der die Nutzenden unterstützen sollen, drei Typen kategorisieret werden können: Die kontextabhängige Hilfe unterstützt die Nutzenden mit wenig Augenmerk auf die Hauptaufgabe der Nutzenden, wodurch diese Art von Unterstützung nicht immer hilfreich ist. Die aufgabenorientierten Hilfe ist eine Weiterentwicklung der kontextabhängigen Hilfe die versucht, die Hauptabsicht der Nutzenden zu erkennen, um diese gezielter zu unterstützen. Die referenzorientierter Hilfe geht nicht direkt auf die Handlungen 
der Nutzenden ein, sondern stellt eine Sammlung von Erklärungen zu den Funktionen des Produkts als Nachschlagewerk zur Verfügung.

Abgesehen von den diesen Unterschieden der Online-Hilfe, variieren diese auch in der Art, wie sie für die Nutzenden erreichbar sind. Dabei lassen sich zwei grundsätzliche Typen von Hilfen unterschieden: Es gibt jene Art von Hilfe, die durch die Initiative der Nutzenden aktiv aufgerufen werden müssen, und jene die ohne viel eigenes Zutun der Nutzenden automatisch präsentiert wird. Zu den aktiven Aufzurufenden zählt beispielsweise Hilfe über Benutzerhandbücher oder Wizards. Zu den automatisch verfügbar gemachten Hilfen zählen On-Screen Instructions, persönliche Agenten sowie Hinweise und Tipps. Hinsichtlich der automatisch angezeigten Unterstützungen, lassen sich noch weitere Spezifizierungen treffen. Wie im Fall von On-Screen Instructions, sind manche Hilfen für die Nutzenden immer präsent und können nicht umgangen werden. Beispielsweise können Hilfetexte die in einer Webseite eingebettet sind, oft nicht ausgeblendet werden. Persönliche Assistenten sind auch stets präsent und geben, sofern die Funktion nicht deaktiviert wird, je nach Bedarf automatisch ihre Vorschläge zur Unterstützung ab. Hinweise und Tipps erscheinen hingegen nur bei bestimmten Handlungen, beispielsweise Maus über einem bestimmten Bereich. Je nachdem wie diese Hilfen den Nutzenden präsentiert werden, können sie demnach mehr oder weniger negativ (beispielsweise als störend, penetrant, ablenkend, .. ) von den Nutzenden wahrgenommen werden.

\subsubsection{Interpretationshilfe und Hypothesen zur Unterstützung von Nutzenden}

Die Interpretationshilfe weist viele Parallelen zu Online-Hilfen auf, da auch sie den Nutzenden bei der Verwendung von Karten helfen möchte. Sie weist ähnliche Eigenschaften wie die eines integrierten Hilfesystems auf, zu dessen Verwendung die Nutzenden verpflichtet werden. Die Interpretationshilfe trägt zudem Eigenschaften von kontextabhängiger Hilfe, indem relevante Informationen direkt ins Interface der Karte eingebettet werden. Ansatzweise kann die Interpretationshilfe auch mit dem Konzept des Wizards verglichen werden, da sie die Kartenutzenden Schritt für Schritt durch die Kartenanwendung führt. Für die Kombination der eben genannten Charakteristiken gibt es unzählige Möglichkeiten in der Umsetzung der Interpretationshilfe. Wie diese für die Interpretationshilfe im Zuge dieser Arbeit zusammengesetzt werden, wird in Kapitel 4.1.2 beschrieben.

Aufgrund der ähnlichen Charakteristik von Online-Hilfen und Interpretationshilfe können die unter Kapitel 2.4.2.3 geäußerten Bedenken zum Einsatz von Hilfesystemen auch bei der Implementierung von Interpretationshilfe in KIT-Karten relevant werden. Vor allem der Umstand, dass Hilfesysteme bei der Aufgabenführung ablenkend und beeinträchtigend wirken, könnte in diesem Zusammenhang von Bedeutung sein. Aufgrund einiger Ähnlichkeiten zwischen der Interpretationshilfe und dem Wizard, können auch die Nachteile dieser Art von Unterstützung auftreten. Durch die Interpretationshilfen in KIT-Karten können Nutzenden dazu verleitet werden, sich nur auf diese zügige und einfache Leitung durch die Karte zu stützen ohne sich auf den dargestellten Inhalt einzulassen. Die von Galitz (2007) und Silver (2004) aufgezeigte Einschätzung mancher Nutzenden, die den Wizard als ineffiziente und frustrierende Anwendungen beschreiben, kann auch bei der Verwendung von KIT-Karten auftreten. Die Nutzenden könnten es beispielsweise bevorzugen, lieber selbstständig die Inhalte zu erkunden, statt an eine Interpretationshilfe gebunden zu sein.

Aus der in der Literatur unteranderem kritischen Reflexion der angewendeten Hilfesysteme lassen sich folgende Hypothesen ableiten, die im Zusammenhang mit Interpretationshilfen in KIT-Karten untersucht werden: 
HYPOTHESE 3 Die Verwendung einer Interpretationshilfe führt zu einem verminderten Explorieren in der Karte. (siehe Kapitel 2.4.1)

HYPOTHESE 4 Die Testpersonen fühlen sich von der Interpretationshilfe bevormundet.

HYPOTHESE 5 Die Interpretationshilfe wird von den Testpersonen als hilfreich und unterstützend wahrgenommen.

\subsection{ZUSAMMENFASSUNG}

Konzepte zur Unterstützung für Nutzende haben den Zweck, die Verwendung einer Anwendung, sei es nun ein KIT-Kartenprodukt oder eine beliebige Software zu erleichtern. Sie bedienen sich dabei verschiedener Ansätze, die den Nutzenden auf unterschiedliche Weise für eine erfolgreiche Benützung behilflich sein soll. Während die in Kapitel 2.4.1 genannten Konzepte sich hauptsächlich mit der Führung der Nutzenden durch Anwendungen beschäftigt, beschreibt Kapitel 2.4.2 allgemein Möglichkeiten, die zur Unterstützung in Softwareprogrammen zur Verfügung stehen. In den unter Kapitel 2.4.1 beschrieben Konzepten wird deutlich, dass eine führende Einflussnahme meist mit einer beschränkteren Verwendung des Produkts einhergeht. Je strikter die Führung ungesetzt ist, desto weniger können die Nutzenden auf eigene Weise in der Anwendung und den Informationen explorieren. Aus den in Kapitel 2.4.2 beschrieben Ansätzen geht hervor, dass Hilfestellungen nicht immer positiv von den Nutzenden angenommen werden. Sind diese nicht ausreichend aufgabenorientiert umgesetzt oder besitzen sie einen zu penetranten Charakter, sind sie mehr störend als hilfreich.

Dieser Überblick der bestehenden Konzepte zur Unterstützung für Nutzende in (Karte-) Anwendungen hilft, die Interpretationshilfe in den bestehenden fachlichen Kontext einzuordnen. Aufgrund ihrer Erscheinung enthält die Interpretationshilfe Ähnlichkeiten mit den beschriebenen Konzepten. Wie in Kapitel 2.4.1.5. und 2.4.2.4 beschrieben, müssen die oben genannten Umstände bei der Implementierung einer Interpretationshilfe bedacht werden. Um den Einfluss der Interpretationshilfe in KITKarten als Steuerung und Unterstützung für die Nutzenden zu untersuchen, wird nun eine empirische Evaluation über die entsprechenden Annahmen vorgenommen. 


\section{METHODISCHE HERANGEHENSWEISE}

Die Herangehensweise zur Beantwortung der Forschungsfrage ist ähnlich der Bewertung eines abgeschlossenen Projekts nach der Frage: Wie gut funktioniert das Produkt? Evaluierende Methoden, die eine "systematische, möglichst objektive Bewertung eines geplanten, laufenden oder abgeschlossen Projektes" (Sarodnick und Brau 2011, 23) ermöglichen, werden für diese Art von Bewertung eingesetzt. Zur konkreten Bewertung der Gebrauchsfähigkeit der Interpretationshilfe in der KITKarte wird eine Usability-Evaluation durchgeführt. Als typische Formen von empirischen Methoden der Usability Evaluierung gelten der Usability-Test und das Fragebogenverfahren (Sarodnick und Brau 2011). Diese ermöglichen gezielte methodische Beobachtung und Befragung der Nutzenden, die zum Erfahrungsgewinn führen. Es wird eine Version der KIT-Karte getestet, die eine Interpretationshilfe enthält und eine, in der diese Komponente ausgespart ist. Die daraus gewonnen Ergebnisse werden vergleichend untersucht und zeigen, inwieweit Interpretationshilfen als geeignete Werkzeuge in KIT-Karten dienen.

Im Folgenden werden nun durch das Testdesign beschrieben, nach welchen Kriterien der Test aufgebaut wird. Die empirischen Methoden geben an, wie die für den Test relevanten Ergebnisse erhoben werden. Das Testsetting beschreibt, in welchem Rahmen der Usability-Test durchgeführt wird.

\subsection{DER USABILITY-TEST}

Der Usability-Test ist ein Forschungsinstrument der klassischen experimentellen Methodik und ist eines der am häufigsten verwendeten Methoden zur Evaluation der Gebrauchsfähigkeiten von Produkten oder Systemen. Durch diese Methoden können Systeme anhand von echten Nutzenden durch realitätsnahe Aufgaben getestet werden. In kontrollierten Situationen wird das Nutzerverhalten beobachtet, Leistungen gemessen und qualitative Informationen gesammelt. Die dabei gewonnenen Beobachtungen, Äußerungen, Interviews oder Messungen lassen auf Probleme und Verbesserungsmöglichkeiten des Systems schließen. Usability-Tests können auf verschiedene Weisen durchgeführt werden. Die Umsetzung reicht von klassisch gehaltenen Experimenten mit großem Stichprobenumfang und komplexen Testdesign bis hin zu sehr informellen quantitativen Ausführungen mit nur einer Testperson. Die Tests können dabei als formative oder summative Evaluation durchgeführt werden. Die formative Evaluation bezieht Nutzende während des Designprozesses in die Produkt- 
entwicklung ein, wodurch die Schnittstelle zwischen Mensch und System iterativ verbessert werden kann. Bei der summativen Evaluation hingegen steht die abschließende Bewertung der Gesamtqualität eines fertigen Systems im Vordergrund. Sie eignet sich weiters, Vergleiche zwischen mehreren alternativen Systemen anzustellen (Sarodnick und Brau 2011).

Die Beantwortung der Forschungsfrage setzt den Fokus auf das Identifizieren von Auswirkungen bei der Verwendung von Interpretationshilfen indem unterschiedliche Systeme einander gegenüber gestellt werden. Folglich ist eine summative Evaluation des Usability-Tests anzuwenden, um die Interpretationshilfe bewerten zu können.

Obwohl der Usability-Test eine geeignete Methode ist, um Aufschlüsse über Nutzerverhalten zu erlagen, weisen Rubin et al. (2008) darauf hin, dass die Aussagekraft der Ergebnisse dennoch mit Einschränkungen verbunden ist. Bei Abhalten eines Usability-Tests wird eine künstliche Situation geschaffen, die nicht vollständig der Realität entsprechen kann. Aufgrund von Annahmen, die für die Testdurchführung getroffen werden müssen, kann der Usability-Test nur als eine Abbildung einer realen Situation verstanden werden. Dieser Umstand muss bei der Interpretation und Übertragung der Ergebnisse berücksichtigt werden. Die Einschränkungen die mit den in diesem Usability-Test angewendeten Methoden verbunden sind, werden in entsprechenden Absätzen der folgenden Kapitel angeführt.

\subsection{TESTCHARAKTERISTIK}

Um für die Beantwortung der Forschungsfrage relevante Ergebnisse zu erlangen, müssen vor der Durchführung des Usability-Tests Entscheidungen über die angewandte Methodik, den Aufbau des Testszenarios und den zu überprüfenden Hypothesen getroffen werden.

\subsubsection{TESTTYP}

Um die Forschungsfrage zu beantworten, werden zwei Szenarioalternativen miteinander verglichen. Dafür wird ein deduktiver Test angewendet, der durch summative Evaluation die Leistungsfähigkeit zweier unterschiedlicher Ausführungen beurteilt (Sarodnick und Brau 2011). Es wird nach dem Zielklassifizierungsansatz "Which is better?" (Gediga und Hamborg 2002) gehandelt bei dem mehrere Systeme hinsichtlich bestimmter Kriterien miteinander verglichen werden. Da die zwei Szenarioalternativen nicht jeweils von denselben Nutzenden getestet werden sollen, wird der Usability-Test nach dem Between Subject Design entworfen. Dieses Design legt fest, dass für jedes Szenario eine eigene Nutzergruppe herangezogen wird, die voneinander unabhängig die verschiedenen Alternativen testet (Sarodnick und Brau 2011).

\subsubsection{TESTSZENARIO}

Bei der Durchführung des Tests ist es wichtig, eine möglichst realitätsnahe Situation zu schaffen. Das geschieht durch Einbetten der Testaufgaben in einen nachvollziehbaren Kontext, sogenannte Szena- 
rios (Rubin, Chisnell und Spool 2008). Die Ausarbeitung der Szenarien kann durch verschiedene Techniken ausgeführt werden und von einer simplen Simulation bis zu einer vollständig ausprogrammierten Anwendung variieren. Beim Usabilty-Testing werden typischerweise Prototypen zum Erproben mit den Nutzenden angewendet. Unter Prototyp versteht man eine erste Ausführung eines Produkts, das in seiner Funktionalität eingeschränkt ist. Prototypen können dabei verschiedene Ausprägungen in Darstellungstreue, Funktionsumfang, Funktionstiefe, Interaktivität, Datengehalt und technische Reife aufweisen was die Funktionsfähigkeiten der Szenarien bestimmt (Sarodnick und Brau 2011; Rubin, Chisnell und Spool 2008).

Um den Einfluss der Interpretationshilfe auf den Informationsgewinn in KIT-Karten zu testen, muss keine vollständig funktionsfähige KIT-Karte vorliegen. Die Auswahl der gebrauchsfertigen Funktionen orientiert sich an der Aufgabenstellung der Szenarien, wodurch nur die relevanten Funktionen umgesetzt werden. Kapitel 4.1.1 beschreibt welche Eigenschaften jedoch essentiell sind und umgesetzt werden müssen.

\subsubsection{HYPOTHESEN}

Im Ansatz der klassischen Methodik werden Hypothesen zur quantitativen Beweisfindung eingesetzt. Hypothesen sind wissenschaftlich formulierte Aussagen über vermutete Ursache-Wirkungszusammenhänge, die den erwarteten Testausgang angeben. Sie sind empirisch überprüfbar und dienen dazu, Testergebnisse quantitativ zu evaluieren. Eine aussagekräftige Formulierung mit möglichst vielen und präzisen Prognosen ist ausschlaggebend, um die Hypothesen nach der Testdurchführung eindeutig bewerten zu können (Rubin, Chisnell und Spool 2008).

Um die im Usability-Test zu testenden Hypothesen herzuleiten, dient die Erarbeitung der wissenschaftlichen Grundlagen durch Kapitel 2. Dieses enthält Recherchen in einschlägiger Fachliteratur und deckt eine Vielzahl der Aspekte ab, die für die Beantwortung der Forschungsfrage relevant sind. Kapitel 3.5. gibt einen Überblick über die Hypothesen, die getestet werden.

Bei der Bewertung der Hypothesen der Falsifikationismus Ansatz von Popper (1935) berücksichtigt. Er besagt, dass Beobachtungen nie die Wahrheit wissenschaftlicher Hypothesen begründen (Verifikation) können, jedoch sehr wohl ihre Falschheit (Falsifikation). Dieser Rechtfertigkeitszusammenhang wird durch die Aussage "alle Schwäne sind weiß" beispielhaft erklärt. Die klassische Sicht der Wissenschaftstheorie würde versuchen, diese Hypothese zu beweisen und aus beobachteten Einzelfällen eine allgemeine Regel abzuleiten. Die Beobachtung eines schwarzen Schwans falsifiziert jedoch die Hypothese ein für alle mal. Denn auch wenn eine Vielzahl an weißen Schwäne aufgespürt werden kann, heißt es noch lange nicht, dass kein schwarzer existiert (Bräuer 2006; Beetz 2013). Wie in Kapitel 3.2.4 ausgeführt wird, wird die Forschungsfrage durch eine kleine Testgruppe evaluiert. Nach klassischer Sicht müsste aus der Beobachtung dieser wenigen Fällen auf eine allgemeine Gültigkeit geschlossen werden, was logisch nicht zulässig ist. Für diese Hypothesenbewertung wird daher die Methode des Falsifikationismus eingesetzt.

\subsubsection{TESTPERSONEN UND TESTUMFANG}

Für die Durchführung des Tests müssen Annahmen zum Testumfang und den daran teilnehmenden Personen getroffen werden. 


\section{a) Testpersonen}

Für die Zusammensetzung der Testgruppe müssen verschiedene Aspekte beachtet werden. Die Testpersonen sollen so gut wie möglich jene Menschen repräsentieren, die in der Praxis ähnliche Anwendungen verwenden. Diese Eigenschaften sollen sich sowohl durch Alter und Geschlecht als auch durch Können und Wissen widerspiegeln. Aus den entsprechenden Personen wird ein Random Sample entnommen (Rubin, Chisnell und Spool 2008).

Bei der Auswahl der Testpersonen wird zwischen zwei Ansprüchen unterschieden: Welche Eigenschaften sind Voraussetzungen, die von den Testpersonen unbedingt mitzubringen sind und welche Eigenschaften sind jene, die die Testpersonen speziell zur Teilnahme am Test qualifizieren (Rubin, Chisnell und Spool 2008). In Kapitel 4.2.2 wird festgelegt, welche Kriterien für die Teilnahme am Usability-Test notwendig sind. Rubin et al. (2008) weisen darauf hin, dass Auswahlkriterien üblicherweise eigenen Annahmen unterliegen. Es muss daher beachtet werden, dass die getroffene Auswahl nicht uneingeschränkt der Realität entspricht. Auch wenn viel Aufwand bei der Definition der Auswahlkriterien für die Testpersonen betrieben wird, können in der Realität dennoch ganz andere Faktoren zur Definition der Zielgruppe entscheidend sein.

Bei der Auswahl der Testpersonen für diesen Usability-Test ist ein weiterer Gedanke entscheidend. MacEachren (1955) und Nielsen (1989) sehen Kartennutzende nicht als homogene Gruppe, sondern verstehen diese als unterschiedliche Nutzergruppen, die sich durch verschiedene Faktoren wie Geschlecht, Alter, Erfahrungen, Hintergrundwissen, etc. voneinander unterscheiden. Diese Unterschiede führen dazu, dass gleiche Information von unterschiedlichen Nutzenden auf unterschiedliche Art wahrgenommen und verarbeitet wird. Als besonders relevanter Unterschied zwischen den Nutzenden gilt der Grad an Hintergrundwissen und ihre Erfahrungen in den für den Test relevanten Themen. Um diesem Umstand Beachtung zu schenken schlägt Ooms (2012) daher eine Strukturierung der Testgruppen vor. Diese soll zwischen einer Gruppe von ExpertInnen, die sich durch ein hohes Erfahrungsniveau bei der Verwendung von Karten auszeichnet und einer Gruppe von Neulingen, die dabei über ein niedriges Erfahrungsniveau verfügen, unterscheiden. ExpertInnen können, im Gegensatz zu Neulingen, durch den hohen Grad an Erfahrungen und der damit verbundenen Menge an Hintergrundwissen, sowohl einfache als auch komplexe Karten effizienter interpretieren. Zur Untersuchung des Nutzerverhaltens ist es daher sinnvoll, zwischen ExpertenInnen und Neulingen zu unterschieden. Kapitel 4.2.2 beschreibt wie dieses für diesen Usability-Test umgesetzt wird.

Um die Personen zu finden, die diesen Kriterien entsprechen, wird ein Eignungstest erstellt. Der Test dient dazu, nur jene Personen zum Usability-Test einzuladen, die die nötigen Eigenschaften mitbringen. Der Eignungstest wird als Online-Test konzipiert, um unkompliziert an interessierte Personen weitergeleitet werden zu können.

\section{b) Testumfang}

Laut Faulkner (2003) besteht ein Zusammenhang zwischen der Anzahl der Testpersonen und gefundener Usability-Problemen. Wie in Tabelle 3-1 abbildet, genügt in der Praxis schon eine relativ geringe Anzahl von fünf bis sechs Testpersonen, um einen hohen Prozentsatz an wesentlichen Problemen aufzudecken.

Sarodnick und Brau (2011) weisen weiters darauf hin, dass auch bei sorgfältiger Auswahl der Testgruppe die einzelnen Personen nicht alle über die gleichen Eigenschaften verfügen, sondern natur- 
gemäß individuelle Unterschiede aufweisen. Dieser Umstand fließt auch in die Testergebnisse ein und muss besonders bei vergleichenden Tests beachtet werden. Um ein repräsentatives und stimmiges Ergebnis zu halten müssen daher genügend Testpersonen in den Usability-Test einbezogen werden (Sarodnick und Brau 2011).

\begin{tabular}{|c|c|c|c|}
\hline $\begin{array}{l}\text { Anzahl } \\
\text { der Personen }\end{array}$ & $\begin{array}{l}\text { Minimal gefundene } \\
\text { Probleme }\end{array}$ & $\begin{array}{l}\text { Durchschnittlich } \\
\text { gefundene Probleme }\end{array}$ & \multirow{8}{*}{$\begin{array}{l}\text { Tabelle 3-1 Anteil gefundener } \\
\text { Usability-Problemen an gesam- } \\
\text { ten bekannten Usability- } \\
\text { Problemen } \\
\text { (eigene Darstellung nach } \\
\text { Faulker, 2003:381) }\end{array}$} \\
\hline 5 & $55 \%$ & $85,55 \%$ & \\
\hline 10 & $82 \%$ & $94,69 \%$ & \\
\hline 15 & $90 \%$ & $97,05 \%$ & \\
\hline 20 & $95 \%$ & $98,4 \%$ & \\
\hline 30 & $97 \%$ & $99,0 \%$ & \\
\hline 40 & $98 \%$ & $99,6 \%$ & \\
\hline 50 & $98 \%$ & $100 \%$ & \\
\hline
\end{tabular}

Diese Arbeit zielt nicht auf ein Ergebnis ab, das durch einen großen Testumfang besticht. Vielmehr sollen erste Einblicke in die Fragestellung erlangt werden, um Tendenzen zu Auswirkungen von Interpretationshilfen abzuleiten. Aufgrund dessen wird ein Testumfang von fünf Personen pro Testgruppe ausgewählt, der es ermöglicht, bereits einen hohen Anteil der auftretenden Usability-Test-Probleme zu identifizieren. Durch die vier insgesamt benötigten Testgruppen ergibt sich ein Gesamtumfang von 20 Testpersonen, die am Test teilnehmen.

\subsection{EMPIRISCHE METHODEN DER DATENERHEBUNG}

Je nach Testzweck sind zum Ergebnisgewinn verschiedene Erhebungsmethoden geeignet. Zu den typischen Erhebungsmethoden in Usability-Tests zählen Verfahren wie Videoaufnahmen, Thinking Aloud oder Eye Tracking-Methoden. Videoaufnahmen eignen sich, um das Verhalten der Nutzenden während des Tests zu beobachten. Thinking Aloud-Methoden ermöglichen Einblicke in die Denkprozesse der Testperson, da diese ihre Gedanken während der Aufgabenstellung verbal äußern. Methoden zur Blickbewegungsmessung, das sogenanntes Eye Tracking, ermittelt mit einer speziellen Software die Bereiche des Bildschirms, die von den Testpersonen wahrgenommen werden (Sarodnick und Brau 2011).

Obwohl jede diese Methoden durchaus ihre Berechtigung hat in diesen Usability-Test angewendet zu werden, werden sie aus überlegten Gründen ausgeschlossen. Der Fokus des durchgeführten Usability-Tests liegt nicht in der Messung der Leistung der Testpersonen während der Anwendung, sondern in der Evaluation der Ergebnisse nach Verwendung der Anwendung. Videoaufnahmen, die das Verhalten während der Testaufführung aufzeichnen, sind daher nicht erforderlich. Die Thinking Aloud 
Methode könnte Informationen über die Verständlichkeit der dargestellten Inhalte wiedergeben. Generell schafft die Anwendung dieser Methode eine unnatürliche und ablenkende Situation (Rubin, Chisnell und Spool 2008). Lautes Denken bedeutet eine Doppelbelastung für die Teilnehmenden, die Konzentration und Zeit erfordert. Quantitative Messungen können aufgrund der auftretenden Verzögerungen verzerrt werden (Sarodnick und Brau 2011). Hauptsächlich wird jedoch gegen diese Methode entschieden, da der Fokus dieser Arbeit nicht auf Evaluierung der Gedanken der Testpersonen liegt, die sie während der Verwendung der KIT-Karte haben. Vielmehr sind die Ergebnisse des anschließenden Wissenstests für die Bewertung der Forschungsfragen ausschlaggebend. Werden die Testpersonen während der Verwendung der KIT-Karte aufgefordert ihre Gedanken auszusprechen, kann dieses auch bei vorgelegter Aufgabenstellung dazu führen, dass die Testpersonen hauptsächlich wenig relevante Informationen (z.B. über Gefallen und Ästhetik, Technische Funktionsfähigkeit) wiedergeben. Wird die Aufforderung zum Aussprechen der Gedanken zu konkret formuliert, besteht die Gefahr, dass die Testpersonen bereits stark beeinflusst in die Verwendung der Karte gehen. Um diesem Umstand nicht hervorzurufen, sollen die Testpersonen die Karte ungestört verwenden. Erst bei den Nachbesprechung der Aufgabenbewertung werden die Gedanken der Testpersonen eruiert.

Eye Tracking wäre für den Test eine durchaus aufschlussreiche Methode, um die Aufmerksamkeitslenkung durch Interpretationshilfen zu untersuchen. Allerdings ist ihre Anwendung mit einem hohen technischen Aufwand verbunden (Sarodnick und Brau 2011). Für eine erste Analyse der zu bearbeitenden Problemstellung wird daher die Aufmerksamkeitsanalyse der Nutzenden anhand der zu lösenden Aufgaben überprüft. Im Zuge weiterer Forschung sollte die Datenerhebung um diese Methode erweitert werden.

Nach Diskussion der Ausschlusskriterien werden nun die für den Test geeigneten Methoden beschrieben. Zur Bearbeitung der Fragestellung ist eine Kombination der drei Methoden Beobachtung, Lösen spezifischer Testaufgaben und Beantwortung eines Fragebogens zielführend. Die Verbindung dieser Methoden ermöglicht es sowohl Performance Data durch Messungen als auch durch den Fragebogen zu gewinnen (Rubin, Chisnell und Spool 2008).

\subsubsection{BEOBACHTUNGEN}

Durch Beobachtungen während des Usability-Tests können auf viele Aspekte des Nutzerverhaltens geschlossen werden. Die in diesem Test angestellten Beobachtungen umfassen das Aufzeichnen des Klickverhaltens und das Messen der Zeit während der Verwendung der KIT-Karte.

Das Erfassen des Klickverhaltens ermöglicht Rückschlüsse auf die Benutzung der Karte. Dadurch wird festgestellt, wie die Teilnehmenden mit der KIT-Karte interagieren. Umgesetzt wird die Bobachtung über die Aufnahme des Bildschirms über das Softwareprogamm Ashampoo Snap 7.0.9. Das Programm speichert eine Videosequenz über alle durchgeführten Aktionen, die anschließend ausgewertet wird. Auch die quantitative Zeitmessung ermöglicht Aussagen über die Intensität mit der sich mit der Karte beschäftigen. Die Zeitmessung erfolgt über zwei definierte Start- und Endpunkte. In Kapitel 4.3.2.2. wird das angewendete Verfahren genauer beschrieben.

\section{Ergebnis \& Einschränkungen}

Die Zeitmessung und das Beobachten des Klickverhaltens bei der Verwendung der Karten stellt ein Kriterium zum Vergleich der Szenarien dar. Die Messergebnisse werden einander gegenübergestellt 
und geben an, wie intensiv sich die Testpersonen in den jeweiligen Szenarien mit den dargestellten Inhalten beschäftigen.

Da die Aufzeichnung des Klickverhaltens ist eine sehr simple Methode ist, sind die Ergebnisse mit einigen Einschränkungen verbunden. Anhand der Klicks soll festgestellt werden, wie viele und welche Karteninhalte aufgerufen werden. Daraus werden Schlüsse über die Intensität der Benützung der Karte abgeleitet. Beachtet werden muss jedoch, dass auch bei einer hohen Anzahl von Klicks - vor allem in Kombination mit einer kurzen Verweildauer in der Karte - die Inhalte nicht zwingend intensiv studiert werden. Umgekehrt kann der Fall eintreten, dass auch bei wenigen Klicks - vor allem in Kombination mit einer langen Verweildauer auf der Karte - die Karte aufwendig interpretiert wird. Dennoch kann durch das Klickverhalten genau beobachtet werden, welche Inhalte von den Testpersonen aufgerufen werden und zumindest Anhaltspunkte zur Intensität abgeleitet werden.

Auch bei der Zeitmessung müssen einige Einschränkungen beachtet werden. Sie misst die Dauer, mit der sich die Testpersonen mit der Karte beschäftigen. Von dieser Information kann nicht vollständig auf die Intensität der Kartennutzung geschlossen werden. Viele weitere Faktoren nehmen Einfluss auf die Zeit, die für das Kartenlesen verwendet wird. Beispielsweise sind generelle Arbeitsweisen, Unterschiede in der allgemeinen Auffassungsgabe, Lesegeschwindigkeit oder Fähigkeiten zur Interpretation von Kartendarstellungen beeinflussend. Diese Umstände müssen bei der Interpretation der Ergebnisse berücksichtig werden.

\subsubsection{TESTAUFGABEN ZUM KARTENINHALT}

Eine weitere Methode um Daten zur Evaluation der Forschungsfrage zu gewinnen ist das Lösen von spezifischen Testaufgaben im Zuge des Usability-Tests. Sie dienen dem zentralen Erkenntnisgewinn und verlangen von den Teilnehmenden bestimmte Inhalte der thematischen Karte zu interpretieren und anschließend wiederzugeben. Für jeden Teilnehmenden ist unabhängig vom zugeteilten Szenario die gleiche Aufgabenstellung zu lösen.

\section{Ergebnis \& Einschränkungen}

Durch Lösen spezifischer Aufgaben und deren Gegenüberstellung wird der Einfluss der Interpretationshilfe untersucht. Die Testlösungen zeigen, wie die Karteninhalte von den Testpersonen interpretiert werden und mit welchem Szenario sie im Zusammenhang stehen. Zu beachten ist dabei jedoch, dass der Erfolg beim Lösen der Aufgabenstellung vermutlich nicht allein vom Vorhandensein der Interpretationshilfe abhängig ist. Faktoren wie Übung im Kartenlesen, allgemeine Auffassungsfähigkeit und weitere können das Testergebnis beeinflussen. Die Validität des Usability-Tests ist daher maßgeblich von der korrekten Auswahl der Testpersonen abhängig (Sarodnick und Brau 2011). In Kapitel 3.4.2. wird erläutert, welche Kriterien bei der Testpersonenwahl zutreffen müssen, um die Verzerrung des Ergebnisses so gering wie möglich zu halten. Auch die differenzierte Betrachtung der Testpersonen und Kategorisierung in Testgruppen hilft, den Einfluss der eben genannten Faktoren so gering wie möglich zu halten. Dadurch können die Einschränkungen zwar nicht vollkommen ausgeschlossen aber immerhin eingrenzt werden. 


\subsubsection{FRAGEBOGEN}

Fragebogenverfahren sind eine der typischen Usability-Evaluationsmethoden. Werden sie begleitend zum Usability-Test eingesetzt, können sie zusätzlich durch Beobachtungen weitere Informationen bringen (Sarodnick und Brau 2011). Zum einen können durch Fragebögen soziodemographische Charakteristiken der Teilnehmenden abgefragt werden, die wichtig sind, um die Kriterien zur Auswahl der Testperson zur überprüfen und hilfreiche Hintergrundinformationen zur Ergebnisinterpretation zu liefern. Weiters sind Fragebögen geeignet um qualitative Informationen wie Gefühle und Meinungen in die Testevaluierung mit einfließen zu lassen (Rubin, Chisnell und Spool 2008).

Je nach Informationen die abgefragt werden, können Fragebögen vor oder nach dem Usability-Test durchgeführt werden. Für diesen Testdurchlauf wird eine Kombination aus Vortest und Nachtest angewendet, die für den Informationsgewinn als Ergänzung zu den spezifischen Aufgabenstellungen eingesetzt werden. Der Vortest dient einerseits dazu, Hintergrundinformationen über den Testteilnehmer zu erfahren. Dieser erlaubt es, die Nutzenden zu klassifizieren und ermöglicht ein größeres Verständnis über die Teilnehmenden, das vor allem bei der Interpretation der Testergebnisse notwendig ist (Rubin, Chisnell und Spool 2008). Der Nachtest besteht aus subjektiven Fragestellungen, die zur Erhebung persönlicher Einschätzungen der Testpersonen dienen. Abgefragt wird hier hauptsächlich das Empfinden während der Aufgabenlösung.

\section{Eigenschaften des Fragebogens}

Auf standardisierte Fragebögen der Usability-Evaluation, beispielsweise nach ISONORM 924/110-S oder IsoMetrics wird verzichtet. Sie erfassen durch mehrstufige Skalen generelle Aspekte zur Systembeurteilung (Sarodnick und Brau 2011). Für die Forschungsaufgabe ist es zielführend, spezifische Fragestellungen zu entwickeln, die mehr Aufschluss über die Auswirkungen von Interpretationshilfen geben.

Um die notwendigen Informationen zu erheben, wird der Fragebogen als eine Kombination aus Multiple-Choice, halboffenen Fragen und Analogskalen konzipiert. Die Verwendung unterschiedlicher Fragetypen erlaubt es, die Informationserhebung den verschiedenen Teilen des Tests anzupassen.

Die Antworten nach dem Multiple-Choice System ermöglichen eine standardisierte Vergleichbarkeit der Ergebnisse. Sie werden bei eindeutigen Fragen eingesetzt, bei denen von den Teilnehmenden eine konkrete Antwort gefragt ist. Das Stellen halb offener Fragen erlaubt es, individuelle Antwortoptionen zu ermöglichen. Sie werden dann eingesetzt, wenn eine Antwort notwendig ist, die differenzierte Rückschlüsse erfordert. Durch sie wird dem Testleitenden die Möglichkeit gegeben, genauer nachzufragen und einen qualitativen Charakter der Befragung zu erzielen. Im Zusammenhang mit der Implementierung der offenen Fragen wird auch die Videoaufzeichnung relevant. Wie bereits in Kapitel 3.3.1 beschrieben ist, wird der Bildschirm über das Softwareprogramm Ashampoo Snap 7.0.9 aufgezeichnet. Dieses Programm ermöglicht auch die Aufnahme von Ton, das ein Mitschreiben der gegeben Antworten zu allen Fragen des Usability-Test überflüssig macht. Teilnehmende und Testleitende können sich dadurch vollständig auf die Fragen konzentrieren, wodurch der Umfang des Gesprächs nicht eingeschränkt wird. Bei besonders umfangreichen und interessanten Antworten kann für die Hypothesenbeantwortung auf die aufgenommenen Videodateien zurückgegriffen werden. 
Generell wird beim Erstellen des Fragebogens auf die wissenschaftlichen Aspekte der Objektivität, Reliabilität und Validität geachtet (Sarodnick und Brau 2011). Weiters wird zur Vermeidung von verzerrten Ergebnissen bei der Formulierung der Fragestellungen auf eine eindeutige und verständliche Sprache geachtet.

\section{Einschränkung}

Bei der Verwendung von Fragebögen ist zu beachten, dass die Ergebnisse, die aus dem Test gewonnen werden können von den darin gestellten Fragen abhängen. Aspekte, die beim Erstellen des Fragebogens nicht berücksichtig wurden, können nur schwer in die Beurteilung der Anwendung einfließen. Der Freiraum der Nutzenden ist vor allem in jenen Bereichen deutlicher eingeschränkt, in denen das Verfahren sehr strukturierter abläuft (Sarodnick und Brau 2011). Aus diesem Grund ist in diesem Test eine Vielzahl von offenen Fragen enthalten, die eine differenzierte Auswertung zulässt. Auch wird am Ende des Tests die Möglichkeit geboten, weitere Kommentare zur Anwendung abzugeben, falls die Testpersonen noch weitere wichtige Anmerkungen machen möchten.

Eine weitere Einschränkung bei der Verwendung von Fragebögen ist, dass die Teilnehmenden dazu neigen könnten, die Fragen nur oberflächlich zu beantworten bzw. zu raten. Diesem kann allgemein nur schwer entgegen gewirkt werden. Es wird jedoch vor Testbeginn an die Teilnehmenden appelliert, sich beim Lösen der Aufgabe ernsthaft Mühe zu geben. Weiters werden die Testpersonen darauf hingewiesen, bei Unsicherheiten nicht zu raten, sondern das entsprechende Feld (z.B. "weiß nicht") zu wählen.

\subsection{TESTSETTING}

Neben der methodischen kann auch die praktische Durchführung von Usability-Tests unterschiedlich ausgeführt werden. Für ein reliabeles Testergebnis sind neben der Auswahl der Testpersonen und der angewendeten Methode zur Datenerhebung, auch Überlegungen zum Testsetting ausschlaggebend.

\subsubsection{TESTUMGEBUNG \& EQUIPMENT}

Generell können Usability-Tests an verschiedenen Orten durchgeführt werden. Stationäre Labore mit aufwendiger Anordnung von Kontrollräumen, Testräumen und Spiegelwände, mobile Laboratorien im Feld oder das direkte Arbeitsumfeld der Testgruppe kommen je nach Testzweck zur Anwendung. Jede dieser Möglichkeiten besitzt Vor- und Nachteile bei der praktischen Anwendung (Sarodnick und Brau 2011; Rubin, Chisnell und Spool 2008). Aufgrund der wenig komplexen Anforderung an den Usability-Test, ist eine aufwändige Ausstattung eines Usability-Labors nicht notwendig. Auf umfangreiche Ausstattungen wie separierte Kontroll- und Testräume kann daher verzichtet werden. Im Rahmen dieser Arbeit ist es vielmehr entscheidend, dass die Testanwendung für alle Testpersonen funktionsfähig und unverändert zur Verfügung steht. Würde die Anwendung auf verschiedenen Computern durchgeführt werden, könnten durch verschiedene Betriebssysteme, Browser und Bildschirmgrößen Verzerrungen in der Darstellung entstehen. Um eine gleiche und vor allem funktionie- 
rende Anwendung für alle Testpersonen zu gewährleisten, ist es in diesem Fall notwendig, den Usability-Test auf demselben Gerät durchzuführen.

Die technische Ausstattung, die zur Testdurchführung notwendig ist, beschränkt sich auf die wesentlichen Notwendigkeiten. Die Evaluierung der Anwendung während des Tests beläuft sich auf das Aufnehmen des Bildschirms und das Ausfüllen des Fragebogens, was einen großen weiteren Aufzeichnungsaufwand (z.B. mit einer Videokamera) ausschließt. Ein Laptop mit entsprechender Software, der vom Testleitenden zur Verfügung gestellt wird sowie die ausgedruckte Fragebogenunterlagen und ein Kugelschreiber sind alles, was benötigt wird. Da das notwendige Equipment sehr gering ist, kann der Usability-Test flexibel an beliebigen Orten durchgeführt werden. Dieses geschieht in Vereinbarung zwischen Teilnehmenden und dem Testleitenden. Da jedoch Ablenkungen und Störeinflüsse gering gehalten werden sollen, muss bei der Wahl des Testorts auf eine entsprechende Umgebung geachtet werden. In jedem Einzelfall wird vom Testleitenden abgewogen, ob die Umgebung als geeignet oder nicht befunden wird.

Folgendes Equipment wird für den Testdurchlauf verwendet:

Laptop:

Lenovo Ideapad U510

Bildschirm: 15.6 Zoll, 16:9,

1366x768 Pixel, spiegelnd
Browser:

Mozilla Firefox: Version 34.0

Modus: Fullscreen (F11)
Software zur Aufnahme:

Ashampoo Snap 7.0.9

\subsubsection{TESTPERSONAL}

Um den Usability-Test durchführen zu können, braucht es eine Person, die den Testpersonen Anweisungen zum Testverlauf gibt. Diese Aufgabe übernimmt der Testleitende, der in seiner Rolle mehr oder weniger aktiv sein kann.

Ist die Rolle des Testleitenden zu aktiv, kann diese das Testergebnis beeinflussen. Zu Verfälschungen kann es bereits durch die Körpersprache des Testleitenden kommen, da diese Einfluss auf das Verhalten der Testpersonen nehmen kann (Sarodnick und Brau 2011; Rubin, Chisnell und Spool 2008). Bei einer passiven Vorgangsweise entsteht der Nachteil, dass wenig Dialog mit den Testpersonen entsteht und zusätzliche wertvolle Informationen nicht gesammelt werden können. Jedoch entspricht diese Situation mehr der Realität, da die Nutzenden die Karte im üblichen Gebrauch auch selbstständig verwenden (Sarodnick und Brau 2011).

Für diesen Test wird ein ausgewogenes Verhältnis zwischen aktiver und passiver Rolle des Testleitenden gewählt. Bei der Befragung wird eine aktive Rolle eingenommen, um viele Informationen über die Hintergründe der Antwort zu erfahren. Während der Verwendung der KIT-Karte wird in eine passive Rolle gewechselt. Um die Testpersonen bei der Bearbeitung der Aufgabe nicht zu stören und damit zu beeinflussen, nimmt der Testleitende eine zurückhaltende Position ein. In seiner passiven Rolle beobachtet der Testleitende des Interaktionsverhaltens der Testpersonen und nimmt dazu Notizen auf Papier. Weiters beantwortet der Testleitende in dieser Phase keine Fragen. Er greift auch nicht in das Aufgabengeschehen ein, soweit es sich nicht um technische Probleme (z.B. bei verse- 
hentlichen Schließen des Browserfensters) handelt. Ob der Test danach für die Evaluierung verwendet wird, wird ggf. in jedem Fall einzeln entschieden.

Für diesen Usability-Test wird die Aufgabe des Testleitenden von der Autorin dieser Arbeit übernommen. Sie erklärt den Testpersonen den Ablauf, startet die Aufzeichnung des Bildschirms und führt die Befragung mittels Fragebögen durch.

\subsubsection{ZEITRAHMEN}

Der zeitliche Rahmen der Testdurchführung soll so kompakt wie möglich gehalten werden. Gleichzeitig soll der Usability-Test aber über genügend Raum verfügen, um die Forschungsfrage stichhaltig beantworten zu können. Für diesen Usability-Test wird ein Zeitrahmen, der 30 Minuten nicht überschreitet gewählt. Durch dieses kompakte Testdesign steigt die Wahrscheinlichkeit, Personen für die Teilnahme am Test zu gewinnen. Die Teilnahme erfolgt auf freiwilliger Basis, bei dem keine Vergütung für den Zeitaufwand an die Teilnehmenden vorgesehen ist.

\subsubsection{PRETEST}

Ist das Design soweit ausgearbeitet, weisen Rubin et al. (2008) darauf hin, den vollständige UsabilityTest einem Pretest (auch Pilottest genannt) zu unterziehen. Dieser stellt sicher, Fehler im Design wie unausführbare Aufgaben, nichtzielführende Datenerhebung überarbeiten zu können. Vor allem für die darin enthaltenen Fragestellungen ist ein Pretest unerlässlich. Durch diesen Schritt wird überprüft, ob die Fragestellungen unvoreingenommen und unmissverständlich formuliert sind und zum gewünschten Informationsgewinn führen. Weiters dient der Pretest dazu, die technischen Funktionsfähigkeiten des Eignungstests und der KIT-Karten zu testen. Neben der Korrektur von Tipp- und Rechtschreibfehlern kann auch der für den Test gesetzten Zeitrahmen im Zuge des Pretest überprüft werden.

Für dieses Testdesign wird der vollständige Testdurchlauf inklusive Eignungstest, Vortest, Anwendungstest und Nachtest einem Pretest durch ein bis drei unabhängige Personen unterzogen. Da nicht alle Teile des Usability-Tests gleich leicht umzusetzen sind, wird der Pretest für manche Teile wiederholt durchgeführt, bis das gewünschte Ergebnis erreicht ist.

\subsection{ZUSAMMENFASSUNG DER HYPOTHESEN UND UNTERHYPOTHESEN}

Wie in Kapitel 2 hergeleitet, wird die Forschungsfrage durch fünf Hypothesen getestet. Diese stellen die Hauptannahmen zur Benützung von Interpretationshilfen in KIT-Karten dar. Wie bereits in Kapitel 3.2.4 beschrieben, besteht aufgrund der heterogenen Eigenschaften von Kartennutzenden die Notwendigkeit, die Testpersonen differenziert zu betrachten. In dieser Arbeit sollen die Kartennutzenden hinsichtlich ihres Grads der Erfahrung in der Verwendung von thematischen Karten unterschieden werden und eine Neulingen- sowie eine ExpertInnen- Gruppe gebildet werden. Die Kriterien der zwei Testgruppen werden in Kapitel 4.2.2 erläutert. Da sich die zwei Testgruppen in ihrer Charakteristik 
stark voneinander unterscheiden, ist ein unterschiedlicher Ausgang in der Hypothesenbewertung zu erwarten. Aus diesem Grund werden Unterhypothesen zu den jeweiligen Hypothesen formuliert, die Erwartungen hinsichtlich der einzelnen Testgruppen treffen.

Neben der Unterscheidung zwischen Neulingen und ExpertInnen soll für eine weiter differenzierte Bewertung der Interpretationshilfe der Einfluss der Interpretationshilfe in bekannten und unbekannten Orten festgestellt werden. Allen Testpersonen wird daher eine bekannte und unbekannte Stadt zur Verwendung vorgelegt, zu denen ein weiter differenzierter Ausgang der Hypothesen vermutet wird. Die Kriterien zur Bekanntheit des Ortes werden in Kapitel 4.2.2 beschrieben.

Bezugnehmen auf die Differenzierungen der Testpersonen zwischen ExpertInnen und Neulingen sowie der Unterscheidung zwischen bekannten und unbekannten Orten zeigt Tabelle 3-2 die Ergänzung durch die entsprechenden Unterhypothesen. Weiters kombiniert diese Tabelle die empirischen Methoden, mit denen diese Hypothesen überprüft werden sollen.

HYPOTHESE

HYPOTHESE 1 Die Verwendung einer Interpretationshilfe führt zur Interpretation von bestimmten Inhalten.

1.1 Nur die von der Interpretationshilfe korrekt genannten Zusammenhänge werden von den Testpersonen richtig erkannt.

1.2 Interpretationshilfe beeinflusst vor allem die Informationsaufnahme der Neulinge.

HYPOTHESE 2 Durch die Verwendung einer Interpretationshilfe werden die dargestellten Inhalte häufiger korrekt interpretiert.

2.1 Die Interpretationshilfe verbessert vor allem die Interpretation der Neulinge.

Testaufgaben zum Karteninhalt

2.2 Der positive Einfluss der Interpretationshilfe tritt vor allem bei unbekannten Orten auf.

HYPOTHESE 3 Die Verwendung einer Interpretationshilfe führt zu einem verminderten Explorieren in der Karte.

3.1 Es werden wenig neue Bereiche neben den von der Interpretationshilfe angesprochenen Bereichen aufgerufen.

Beobachtungen

3.2 Ist eine Interpretationshilfe vorhanden verbringen die Testpersonen weniger Zeit mit der Verwendung der Anwendung.

3.3 Die Interpretationshilfe vermindert vor allem das Explorieren der Neulinge. 
HYPOTHESE 4 Die Testpersonen fühlen sich von der Interpretationshilfe bevormundet.

Fragebogen

4.1 Die Interpretationshilfe wird hauptsächlich von ExpertInnen negativ beurteilt.

HYPOTHESE 5 Die Interpretationshilfe wird von den Testpersonen als hilfreich und unterstützend wahrgenommen.

Fragebogen

$+$

Testaufgaben

zum

Karteninhalt

5.2 Die Interpretationshilfe wird vor allem bei unbekannten Orten als Hilfe empfunden.

Tabelle 3-2 Überblick über Hypothesen und Unterhypothesen sowie anzuwendender Methode (eigene Darstellung)

Nachdem in diesem Kapitel alle grundsätzlichen Entscheidungen zur methodischen Herangehensweise getroffen wurden, wird im nun folgenden Kapitel die genaue Konzeption zur Überprüfung der Hypothesen durch den Usability-Test beschrieben. 


\section{EMPIRISCHE EVALUATION}

Im Zuge der empirischen Evaluation beschäftigen sich die Testpersonen mit einer komplexen interaktiven thematischen Karte. Anhand dieser Karte gilt es eine Aufgabenstellung zu bearbeiten, die durch gezieltes Ablesen und Interpretieren von Informationen gelöst werden kann. Um den Einfluss der Interpretationshilfe auf die Informationsaufnahme zu bewerten, werden zwei unterschiedliche Szenarioalternativen entworfen. Die Umsetzung beider Varianten ist hinsichtlich allgemeiner Benutzeroberfläche und dargestellter Karteninhalte ident. Der Unterschied der Szenarien liegt lediglich im Vorhandensein einer Interpretationshilfe, die von den Nutzenden zu verwendet ist.

Um durch den in der empirischen Evaluation eingesetzten Usability-Test verwendbare Ergebnisse zu erheben, müssen bei der Konzeption bestimmte Kriterien beachtet werden .Die eingesetzte KIT-Karte muss nach bestimmten Kriterien umgesetzt werden und ihre Inhalt und die Implementierung in Abstimmung auf die zu testenden Hypothesen erfolgen. Auch die Testpersonen, die diese Anwendung testen, müssen bestimmte Voraussetzungen erfüllen. Bei der Konzeption des Testdurchlaufs wird weiters darauf geachtet, dass die erhobenen Daten auch tatsächlich zu Ergebnissen führen, die für die Beantwortung der Hypothesen relevant sind. Nur dann kann in weiterer Folge die Forschungsfrage beantwortet werden.

In diesem Kapitel werden nun die einzelnen Schritte und Entscheidungen beschrieben, die durch die empirische Evaluation zur Beantwortung der Forschungsfrage führen.

\subsection{DIE KARTENWENDUNG}

Wie Kapitel 2.2 und Kapitel 2.3 zeigen, sind beim Lesen der Karte viele Faktoren involviert, die den Kommunikationsprozess zwischen Kartenersteller und Kartenleser beeinflussen. Wird eine Karte zur Übermittlung einer bestimmten Nachricht erstellt, kann diese modifiziert beim Leser eintreffen. Nutzende können vor allem bei Karten mit komplexen Inhalten damit überfordert sein, die dargestellten Informationen sinnvoll zu verwerten. Dennoch werden Karten, vor allem in der derzeit beliebten Form als interaktive Datenvisualisierungen, gerne eingesetzt, um eine hohe Dichte an Informationen eindrucksvoll zu präsentieren. Interaktivität lässt die Karte dabei zu einem komplexen kartographischen Produkt werden, das viele Funktionen zur Interaktion mit den Inhalten bietet. Kapitel 2.4 zeigt, 
dass bei komplexen (Karten-) Anwendungen verschiedene Methoden und Werkzeuge angewendet werden können, um die Nutzenden bei der Verwendung des Produkts zu unterstützen.

Als Beispiel einer Karte, die für ihren komplexen Inhalt, eine Hilfe für die Nutzenden bietet, ist eine Karte die auf der Nachrichtenwebseite ZEIT ONLINE veröffentlicht wurden. Mit dem Titel "Gesundheitswesen: Wie die Medizin dem Geld folgt" stellt sie die Verteilung der ÄrztInnen in deutschen Großstädte dar. Die Karte wurde im Zusammenhang mit dem Artikel "Reich ist gesund, arm bleibt krank" im April 2014 veröffentlicht. Abbildung 4-1 zeigt einen Ausschnitt dieser Karte. Die interaktive Karte wird im Kontext dieser Webseite dazu eingesetzt, die im Text vorgebrachten Argumente zu unterstützen. Weiters bietet sie den Lesenden des Artikels die Möglichkeit, sich selbst mit der Thematik auseinander zu setzen. Als Hilfestellung in dieser Karte wird eine sogenannte "Tour" angeboten, die die Nutzenden anhand weniger Klicks durch die Karte führt und dabei wesentliche Inhalte erklärt. Ausgehenden von diesem Beispiel soll nun im Zuge dieser Arbeit der positive Einfluss von Nutzerunterstützungen in interaktiven Karten getestet werden. Dafür werden für die komplexen, interaktiven, thematischen Karten bestimmte Eigenschaften festgelegt, die als KIT-Karte definiert werden. Für die Unterstützung, die Nutzenden zur Verfügung steht, werden bestimmte Kriterien festgelegt, die der sogenannten Interpretationshilfe entsprechen.

Im Folgenden wird nun beschrieben, nach welchen Kriterien die Umsetzung der KIT-Karte und der Interpretationshilfe erfolgt.

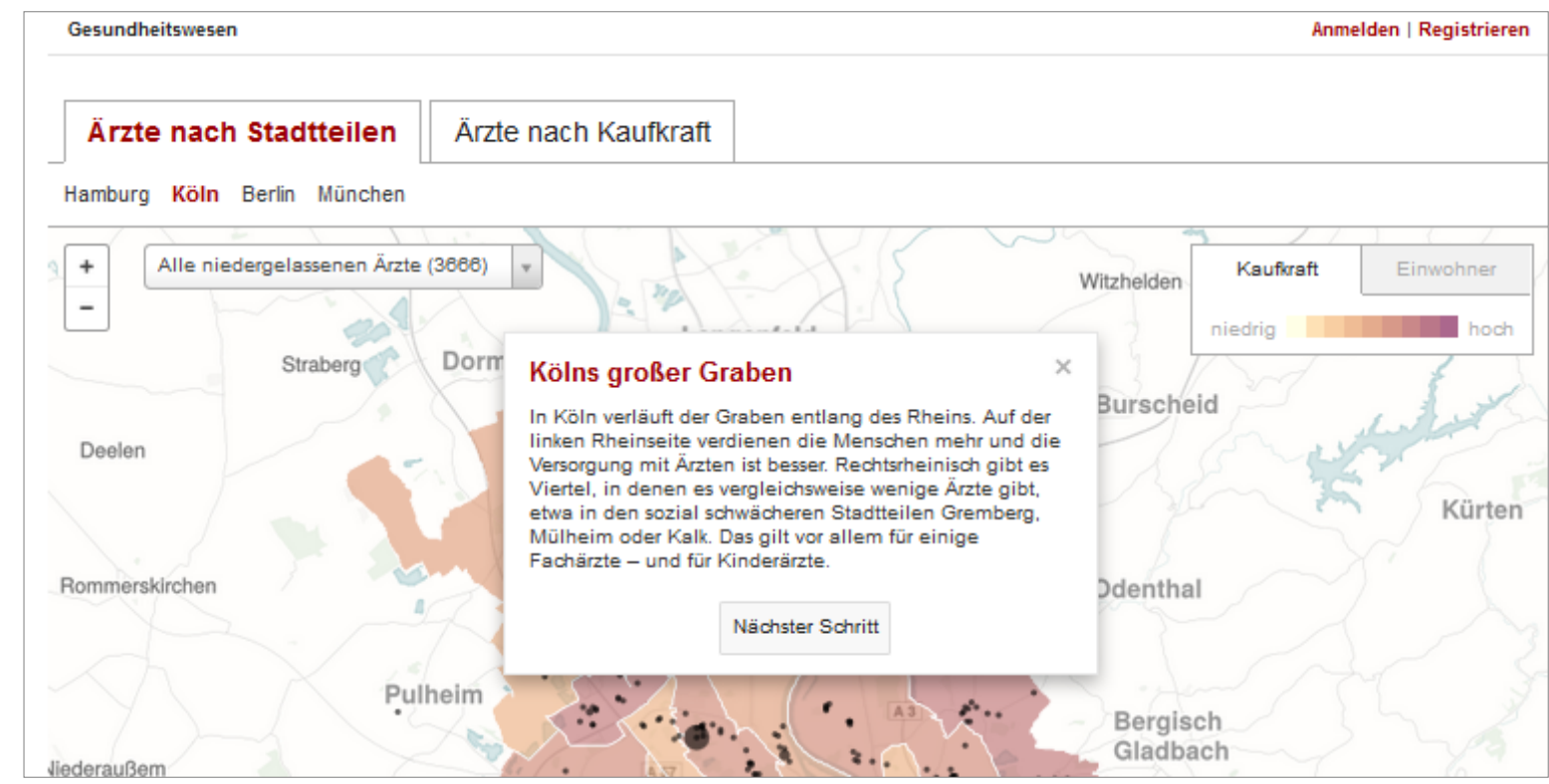

Abbildung 4-1 ZEIT ONLINE Karte mit aktivierter Tour zur Unterstützung der Kartennutzenden (Screenshot ZEIT ONLINE 2014)

\subsubsection{IMPLEMENTIERUNG DER KIT-KARTE}

Die für den Usability-Test entwickelte Karte soll möglichst genau den unter Kapitel 1.2.4.2 und Kapitel 2.1 beschriebenen Kriterien und Eigenschaften entsprechen. Bestimmte konzeptionelle, inhaltliche und technische Ansprüche müssen berücksichtigt werden. Der generelle Aufbau der Karte erfolgt in Anlehnung an das oben beschriebene Kartenbeispiel von ZEIT ONLINE. 


\subsubsection{Konzeptionelle Ansprüche}

Wie die Definition der KIT-Karte voraussetzt, ist zum einen die Komplexität eine der beschreibenden Eigenschaften. Für die Umsetzung der Karte sollen daher thematische Aussageebenen dargestellt werden, die sinnvoll miteinander kombiniert werden können. Zur thematischen Überlagerung eignet sich die Kombination von unterschiedlichen Typen von Signaturen. Beispielsweise kann eine flächenhafte Aussage durch Dichtemosaike in Verbindung mit den Punktsignaturen dargestellt werden. Zur Orientierung soll es weiters eine Basiskarte geben, die die thematischen Inhalte ergänzt. Neben der klassischen Darstellung der Daten durch Karten sollen diese auch durch Diagramme visualisiert werden.

Als weitere unerlässliche Eigenschaft der KIT-Karte gilt die Interaktivität. Die für den Usability-Test entwickelte Karte kann, wie in Kapitel 3.2.2 beschriebenen, als Prototyp umgesetzte werden. Es müssen daher nicht alle in Kapitel 1.2.4.2 genannten Eigenschaften zu interaktiven Karten vollständig umgesetzt werden. Eine Auswahl der für das Testszenario wichtigen ist ausreichend. Funktionen zum Ein- und Ausblenden von Inhalten sowie das Filtern von Informationen und die Möglichkeit bei Mouse-Over weitere Informationen offen zu legen sind demnach als wichtige interaktive Elemente umzusetzen.

\subsubsection{Auswahl der Inhalte}

Um die definierten konzeptionellen Ansprüche umzusetzen zu können, muss eine Thematik gewählt werden, die dieses auch ermöglicht. Das genannte Beispiel von ZEIT ONLINE zeigt eine Idee, welche Inhalte sich für eine sinnvolle Verknüpfung eignen. In Anlehnung an dieses Beispiel werden auch für die Umsetzung der KIT-Karte die Standorte von Arztpraxen als Punktdaten verwendet. Sie sind deswegen gut geeignet, da sie üblicherweise in großer Zahl in Städten vertreten sind und daher städträumlich relevant sind. Weiters kann bei der Darstellung von Arztpraxen aufgrund der verschiedenen Fachgebiete eine differenzierte Darstellung erfolgen. Als flächenhafte Aussage werden für die Karten Informationen zu Einwohnerdichte, durchschnittlichem $\mathrm{m}^{2}$-Preis für Eigentumswohnungen und Einkommensniveau der Bevölkerung dargestellt. Diese wurden gewählt, da sie demographische stadträumliche Charakteristiken abbilden.

Um die Karte nicht mit nebensächlichen Inhalten zu überladen, wird die Grundkarte auf das Wesentliche reduziert. Die Darstellung der Baublöcke und des Straßennetzes hilft, einen Eindruck über die bauliche Struktur der Stadt zu erlangen. Weiters stehen Informationen zur räumlichen Ausdehnung der Bezirke, sowie deren Namen zur Verfügung. Abbildung 4-2 zeigt am Beispiel von Kopenhagen die in der KIT-Karte enthaltenen Inhalte.

Die Visualisierung dieser Daten soll anhand von verschiedenen Großstädten durchgeführt werden. Die Darstellung von nur einer Stadt ist für die KIT-Karte zu wenig komplex. Mindestens zwei Städte sind notwendig, um die verschiedenen Aussagen miteinander kombinieren zu können. Die Auswahl der Städte erfolgte nach zwei Kriterien. Zum Einen ist es notwendig, dass die Daten der jeweiligen Stadt in entsprechender Datenqualität vorhanden sind und möglichst offen und frei zugänglich sind. Zum Anderen soll es weiters möglich sein, differenzierte Aussagen zur Verwendung der Interpretationshilfe treffen zu können. Deshalb wird eine Stadt gewählt, mit denen die Testpersonen vertraut sind und eine Stadt zu der kein Bezug besteht. Als bekannte Stadt wird Wien herangezogen, da angenommen wird, dass die Testpersonen mit den administrativen Grenzen und der räumlichen Ausdehnung vertraut sind. Als Beispiel einer unbekannten Stadt wird Kopenhagen ausgewählt, da die Test- 
personen vermutlich keinen näheren Bezug zu den administrativen, geographischen Eigenschaften sowie weiteren Bevölkerungsindikatoren dieser Stadt haben. Ob diese beiden Kriterien auch von den Nutzenden wahrgenommen werden, wird im Eignungstest sichergestellt.

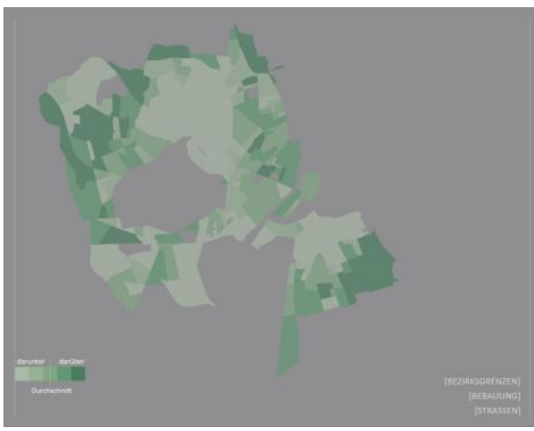

$\varnothing$ NETTOEINKOMMEN PRO PERSON (€/JAHR)

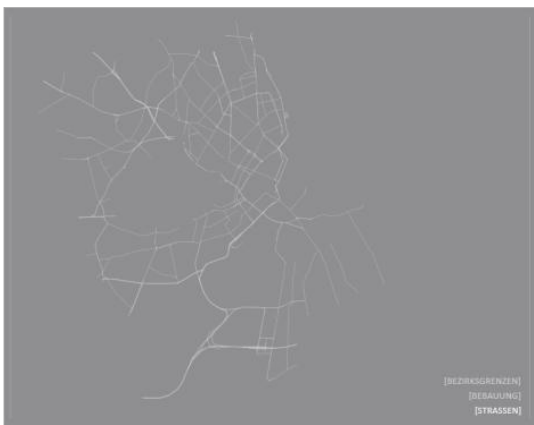

STRASSEN

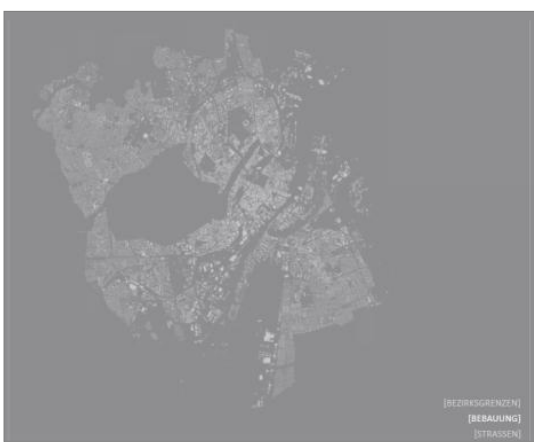

BEBAUUNG

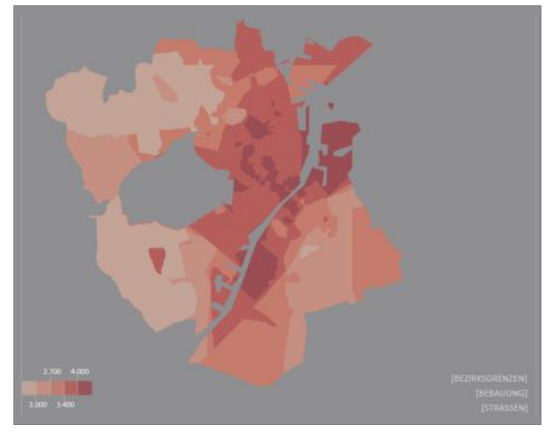

$\varnothing$ KOSTEN EIGENTUMSWOHNUNG (€/M²)

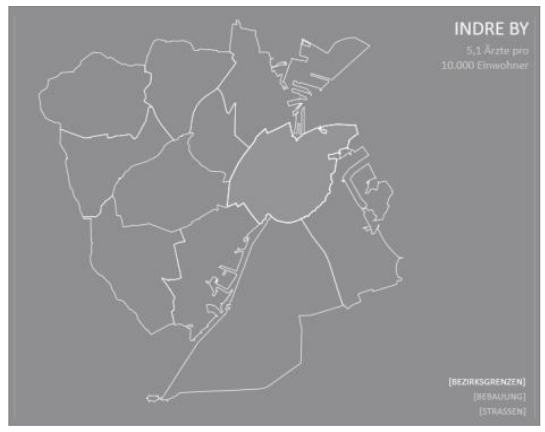

BEZIRKSGRENZEN

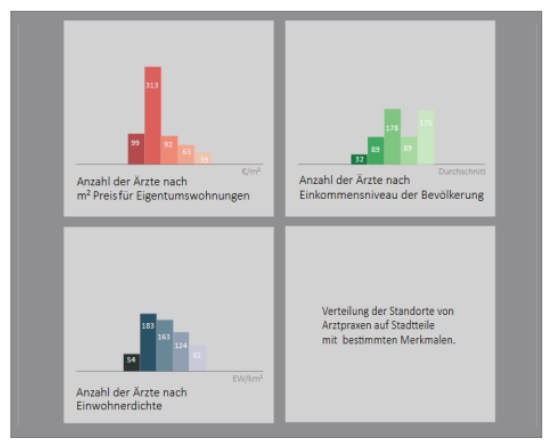

STATISTIK

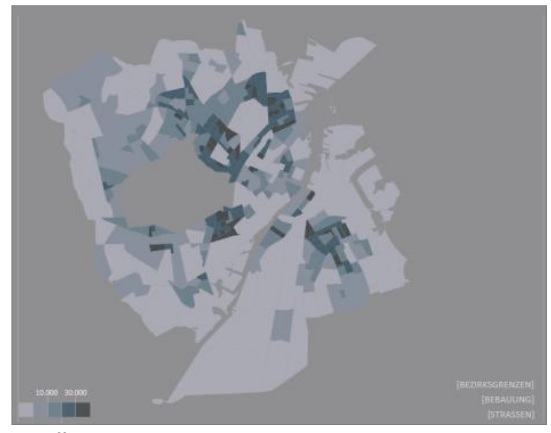

BEVÖLKERUNGSDICHTE (EINWOHNER/KM²)

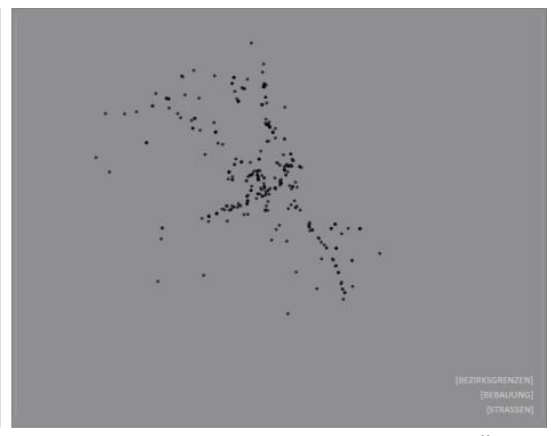

FACHÄRZTE

Abbildung 4-2

Inhalte der KIT-Karte am Beispiel

Kopenhagen

(Screenshots KIT-Karte)

\subsubsection{Technische Umsetzung}

Für die technische Umsetzung werden die gemeindebezogenen Daten hauptsächlich von den Open $D_{a t a}{ }^{2}$ Seiten der jeweiligen Städte bezogen. Ergänzt wurden diese Daten mit bereits veröffentlichten Karten zu relevanten Themen. Da in diesen Datensätzen keine Informationen zur Verortung von Arztpraxen zur Verfügung stehen, wurden diese von den entsprechenden Seiten der Gemeinden bezogen. Eine detaillierte Übersicht der Datenquellen findet sich im Anhang A. Aufgrund der unterschiedlichen Organisation des Ärztewesens in Österreich und Dänemark ist es schwierig alle Adressen vollständig zu finden und zu einer einheitlichen Kategorisierung nach Facharztgruppen zusammenzufügen. Stichprobenartig wurden vor allem für Dänemark die herangezogene Quellen mit weiteren

\footnotetext{
${ }^{2}$ freie und kostenlose Verfügbar- und Nutzbarkeit von Daten
} 
(z.B. elektronisches Telefonbuch, Brancheneintragungen in Google Maps, ..) stichprobenartig verglichen.

Zur Erstellung der Karten werden die Adressen der ÄrztInnen durch den Mapping-Dienst GPS Visualizer mit Koordinaten versehen. Gemeinsam mit den Gemeindedaten werden sie durch das GISProgramm qGIS als Kartendarstellung visualisiert um anschließend in Illustrator exportiert zu werden. Dort werden die Daten mit weiteren Inhalten kombiniert, die für diesen Zweck von bereits bestehenden Karten digitalisiert wurden. Nachdem die einzelnen Layer im Illustrator zu sinnvollen Karten zusammengestellt und mit einem Kartenlayout versehen wurden, werden die Karten durch JavaScript und $H T M L / C S S$ mit interaktiven Elementen ausgestattet.

Um die Daten zur Darstellung in den Diagrammen aufzubereiten, wurden je nach Datenlagen Berechnungen mit $q G I S$ oder durch einfaches Abzählen durchgeführt. Die Diagramme wurden anschlieBend in Microsoft EXCEL erstellt, in Adobe Illustrator übertragen und weiters mit Mouse-OverEffekten versehen.

\subsubsection{IMPLEMENTIERUNG DER INTERPRETATIONSHILFE}

Die implementierte Interpretationshilfe ist das Hauptthema dieser Arbeit. Wie in Kapitel 1.2.4.3 beschrieben, übernimmt sie die Aufgabe, die Kartennutzenden bei der Benützung der Karte zu unterstützen. Um diesem Anspruch gerecht zu werden, müssen bei der Umsetzung der Interpretationshilfe bestimmte Aspekte beachtet werden.

\subsubsection{Konzeptionelle Ansprüche}

In Kapitel 2.4.1.6 und Kapitel 2.4.2.5 werden die wesentlichen Eigenschaften der Interpretationshilfe in Bezug auf bestehende Konzepte der Nutzerunterstützung beschrieben. Elementar ist dabei die Sicherstellung, dass bestimmte Zusammenhänge und Informationen auf jeden Fall von den Nutzenden wahrgenommen werden und die von den Kartenautoren vorgesehene Aussage der Karte kommuniziert wird. Eine Einbettung der Interpretationshilfe als Schritt-für-Schritt-Führung direkt ins Interface der Karte stellt sicher, dass keine von den Kartenautoren als relevant eingestufte Information von den Nutzenden unaufgerufen beleibt.

\section{a) Einbettung in das Interface}

Zur direkten Einbettung in der Interpretationshilfe ins Interface der Anwendung gibt es verschiedene Möglichkeiten. Je nach Art und Weise der Umsetzung ergeben sich unterschiedliche Auswirkungen auf ihre Verwendung. Die Implementierung kann verschiedene Varianten zwischen unauffällig neben der Karte bis hin zu auffällig die Karte überlagernd annehmen. Für diese Arbeit soll ein Mittelweg gewählt werden, durch den die Interpretationshilfe nicht zu dominant aber auch nicht zu sehr im Hintergrund erscheint. Das Angebot der Interpretationshilfe soll von den Nutzenden eindeutig wahrgenommen werden können, gleichzeitig jedoch die Wahrnehmung der Karten- und Diagramminhalte nicht behindert werden.

Die Interpretationshilfe wird dafür als Textfeld dezent, aber dennoch gut sichtbar, neben den Darstellungen der KIT-Karte platziert. Nachdem es um die Kartendarstellungen keinen weiteren Text gibt, 
sollte sie eindeutig wahrzunehmen sein. Abbildung 4-3 zeigt die Einbettung dieser Funktion in die KIT-Karte.

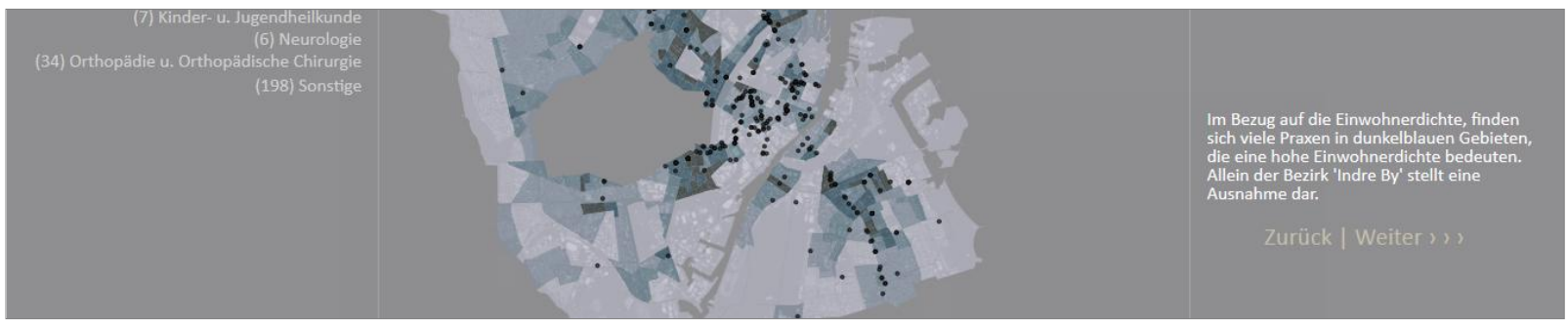

Abbildung 4-3 Implementierung der Interpretationshilfe im Interface KIT-Karte

(Ausschnitt Screenshot)

\section{b) Verpflichtung der Nutzenden}

Eine weitere Charakteristik der Interpretationshilfe ist ihre verpflichtende Verwendung. Diese stellt eine autorengesteuerte Kontrolle über die präsentierten Inhalte sicher. Gleichzeitig sollen die Testpersonen jedoch die Möglichkeit haben, in der jeweils dargestellten Karte weiter explorieren zu können. Interaktiven Funktionen wie Mouse-Over-Effekte, Ein- und Ausblenden von thematischen Ebenen sowie das Wechseln zwischen den Karten- und Diagrammdarstellungen bleibt demnach möglich. Für diesen Prototyp der KIT-Karte wird die Interpretationshilfe dadurch verpflichtend gemacht, dass die Teilnehmenden des Szenarios explizit dazu aufgefordert werden, diese zu verwenden und ihren Anweisungen zu folgen. Sollten sie während der Verwendung von dem von der Interpretationshilfe vorgeschlagenen Pfad abweichen, kann mit der "Zurück"-Taste auf der Tastatur wieder zum entsprechenden Punkt zurückgekehrt werden. In einer weiter entwickelten Version sollte die Interpretationshilfe technisch so eingebettet werden, dass auch beim Wechseln des Inhalts (z.B. zwischen Karten- und Diagrammdarstellung) die textlichen Kommentare nicht verschwinden. Zu jedem Zeitpunkt soll es auch bei verändertem Inhalt möglich sein, die Führung fortsetzen zu können.

\section{c) Schritt-für-Schritt-Führung}

Die Interpretationshilfe soll die Aufgabe übernehmen, die Nutzenden "an der Hand zu nehmen" und zu relevanten Inhalten der KIT-Karten führen. Dieses wird durch einen „Weiter"-Button realisiert, der die Testpersonen von einer Darstellung zur Nächsten führt. Mit jedem Klick ändert sich der Inhalt der textlichen Kommentare und dazu entsprechend die ein- und ausgeblendeten Aussageebnen der Karte. So kann genau festgelegt werden, welche Aussageebenen in welcher Kombination mit welchem erklärenden Text präsentiert werden.

\subsubsection{Auswahl der Inhalte}

Der Inhalt der Interpretationshilfe ist kein allgemeiner Text, der das Thema der Karte zusammenfassend beschreibt. Vielmehr steht der Inhalt der Interpretationshilfe in konkreten Zusammenhang mit dem dargestellten Inhalt der Karte. Um diesem Ausdruck zu verleihen, werden relevante Inhalte der Karte kombiniert und für die jeweilige Kartenaussage unwichtige Komponenten ausgeblendet.

Der Inhalt der Interpretationshilfe versteht sich als aggregierte und explizite Aussage, die den Inhalt der Kartendarstellung durch einen kurzen klaren Satz auf den Punkt bringt. Wie bereits in Kapitel 1.2.4.3 beschrieben, stellt die Interpretationshilfe dabei kein weiteres Hintergrundwissen zur Verfü- 
gung, sondern enthält Aussagen, die sich auf die Zusammenhänge der dargestellten thematischen Ebenen stützen. Die konkreten Inhalte werden so gewählt, dass sie den zu beantwortenden Hypothesen Antwort stellen können.

\subsubsection{Technische Umsetzung}

Die Interpretationshilfe wird durch HTML Textelemente umgesetzt. Bei Drücken des "Weiter"Buttons wird jeweils eine neue HTML Seite geladen, in der die JavaScript Eigenschaften der KIT-Karte so verändert sind, dass die zum Text der Interpretationshilfe entsprechenden Inhalte in der Karte anzeigt werden. Weiters steht ein "Zurück"-Button zur Verfügung, der für eine erhöhte Benutzerfreundlichkeit der Interpretationshilfe sorgt. Auch für die Karten zu Wien und Kopenhagen sowie zu den dazugehörigen Diagrammdarstellungen werden neue HTML Seiten erstellt, die durch Links miteinander verbunden werden.

\subsubsection{DAS FERTIGE PRODUKT}

Die Nutzenden haben nun die Möglichkeit jeweils für Wien und Kopenhagen zwischen Kartendarstellung ("Karte") und Balkendiagrammen ("Statistik") zu wechseln. Links neben der Karte stehen die verschiedenen Facharztgruppen durch Anklick zur Anzeige in der Karte bereit. Auch ist dort die Auswahl für die anzuzeigende Choropletenkarten zu finden. Bei Bewegung der Maus über die Karte erscheinen Informationen zum Bezirk, die die administrative Grenze, Bezirksname und Anzahl der ÄrztInnen pro 10.000 Einwohner enthalten. Weiters finden sich in den Kartenrahmendaten Informationen zu Quellen und Kartenerstellerin.

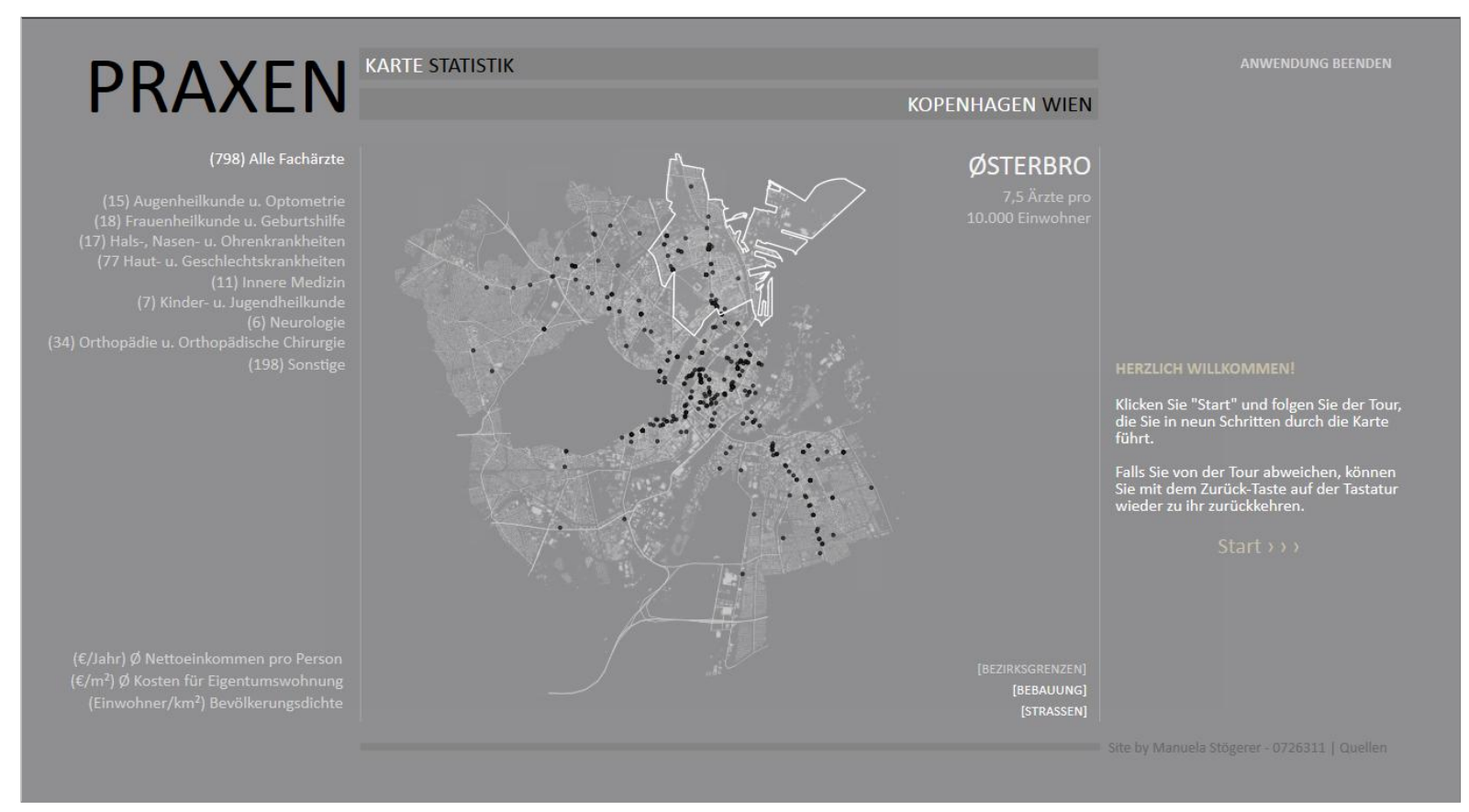

Abbildung 4-4 KIT-Karte mit Interpretationshilfe und aktiver Mouse-Over-Funktion (Screenshot)

Wie in Kapitel 3 erläutert, sind zur Beantwortung der Forschungsfrage zwei Szenarien notwendig, die miteinander verglichen werden. Der Unterschied dieser Szenarien besteht im Vorhandensein der 
Interpretationshilfe. So steht in Szenario 1 rechts neben der Karte die Interpretationshilfe zur Verfügung. In Szenario 2 wird hingegen an dieser Stelle nichts angezeigt. Abbildung 4-4 zeigt die vollständige Karte in Szenario 1. In der Abbildung ist der erste Schritt der durch die Interpretationshilfe festgelegten Führung durch die Karte zu sehen. Die weiße Schrift lässt erkennen, dass für diese Darstellung die Inhalte zu "Alle Fachärzte", "Bebauung" und "Straßen" für Kopenhagen aktiv sind.

Die Anwendung wird nun dazu eingesetzt die Hypothesen durch den Usability-Test zu prüfen. Welche Kriterien für die Umsetzung dieses Tests notwendig sind, wird im folgenden Kapitel beschrieben.

\subsection{TESTKRITERIEN}

Kapitel 3 beschreibt die methodische Herangehensweise mit der der Usability-Test zur Beantwortung der Forschungsfrage konzipiert wird. In diesem Kapitel werden nun weitere Kriterien zur Umsetzung des Usability-Tests getroffen. Annahmen über den Testumfang des Usability-Tests, den von den Testpersonen zu erfüllenden Kriterien und die Rekrutierung dieser sind wichtige Überlegungen.

\subsubsection{TESTUMFANG}

Kapitel 3.2.4. beschreibt bereits, dass die Auswahl der Testpersonen maßgeblich entscheidend für den Ausgang des Tests ist. Dieses betrifft sowohl die Anzahl der herangezogenen Personen als auch ihre Eigenschaften. Wie Tabelle 4-1 zeigt, besteht dieser Test aus zwei Szenarien, die jeweils von zwei Testgruppen getestet werden. Daraus ergeben sich vier Testgruppen, die mit einer entsprechenden Anzahl von Testpersonen zu besetzen sind. In Bezug auf Falkner (2003) werden pro Testgruppe fünf Personen herangezogen, um ein gewisse Repräsentativität der Testgruppe sicherzustellen. Es ergibt sich daher ein Testumfang von insgesamt 20 teilnehmenden Personen.

\begin{tabular}{|c|c|c|c|}
\hline & $\begin{array}{c}\text { SZENARIO } 1 \\
\text { mit Interpretationshilfe }\end{array}$ & $\begin{array}{c}\text { SZENARIO } 2 \\
\text { ohne Interpretationshilfe }\end{array}$ & \multirow{3}{*}{$\begin{array}{l}\text { Tabelle 4-1 } \\
\text { Testgruppen des Usability-Tests } \\
\text { (eigene Darstellung) }\end{array}$} \\
\hline NEULINGE & 5 Personen & 5 Personen & \\
\hline EXPERTINNEN & 5 Personen & 5 Personen & \\
\hline
\end{tabular}

\subsubsection{QUALIFIKATIONEN DER TESTPERSONEN}

Wie in Kapitel 3.2.4 beschrieben, müssen die Testpersonen zur Teilnahme am Usability-Test bestimmte Eignungskriterien erfüllen. Diese beziehen sich sowohl auf körperliche Voraussetzungen, allgemeine Bildungskriterien, Verwendung von Computer und Internet als auch Ortskenntnisse. Das Festlegen dieser Kriterien ist notwendig, um die allgemeine Verständlichkeit der Aufgabenstellung zu gewährleisten und annähernd gleiche Voraussetzungen zur Teilnahme zu schaffen. Weiters wird die 
Vertrautheit in der Verwendung von thematischen Karten als Kriterium zur Differenzierung der Testgruppen herangezogen.

In Punkt a) werden jene Kriterien aufgelistet, die von allen Testpersonen erfüllt werden müssen, die am Usability-Test teilnehmen möchten. Diese voraussetzenden Kriterien werden weiters durch personenbezogene Daten (z.B. Beruf, Arbeitsort) ergänzt, die die Testergebnisse für die Interpretation gezielter einordnen lassen. Abgesehen von diesen Grundvoraussetzungen zur Teilnahme am Test werden in Punkt b) weitere spezifische Kriterien festgelegt, die es ermöglichen, die Testergebnisse differenzierter zu betrachten. Diese beziehen sich auf die Erfahrungen und Kenntnisse der Testpersonen im Umgang mit thematischen Karten.

\section{a) Voraussetzung für alle Testpersonen}

\section{Geschlecht:}

Um eine ausgeglichene Zusammensetzung der Testgruppe zu gewährleisten, soll die Anzahl an männlichen und weiblichen Testpersonen ausgeglichen sein und jeweils $50 \%$ des jeweiligen Szenarios ausmachen.

\section{Beruf:}

Um Vorteile bei der Aufgabenlösung für bestimmte Personen auszuschließen, werden Personen, die eventuell über fachspezifisches Hintergrundwissen verfügen, vom Test ausgeschlossen. Das ist notwendig, um das Vorwissen auf einem ähnlichen Level zu halten. Nicht am Test teilnehmen darf daher die Berufsgruppe der ÄrztInnen.

Alter:

Um externe Faktoren, die altersbedingt zustanden kommen könnten, zu exkludieren, wird das Alter der Testteilnehmenden eingegrenzt. Durch ein ähnliches Alter der Testpersonen werden die Unterschiede innerhalb der Testgruppen so gering wie möglich gehalten. Es wird angenommen, dass Personen der gleichen Altersgruppe tendenziell ein ähnliches Verhalten im Bezug zu Computer und Internet haben, da sie alle gleich intensiv mit dieser Technik aufgewachsen sind. Diese Behauptung wird allerdings weiter durch Einschätzungen bezüglich der Computerverwendung festgestellt. Die Testpersonen sollen daher um die 25 Jahre alt sein, wodurch sich mit einem Spielraum von +/- 3 Jahren eine Spanne von 22 bis 28 Jahren ergibt. Diese Gruppe gilt als besonders interessant, da diese Altersgruppe gerade ins Berufsleben einsteigt bzw. eingestiegen ist.

\section{Seheinschränkungen:}

Um die Karten auch visuell vollständig wahrnehmen zu können, dürfen die Testpersonen nicht unter starken Seheinschränkungen leiden (z.B. Farbenblindheit, hochgradiger Astigmatismus). Diese Kriterien werden durch Selbsteinschätzung der Kandidaten festgestellt.

\section{Deutschkenntnisse:}

Um sprachlich bedingte Verständnisschwierigkeiten auszuschließen, müssen die Testpersonen über fließende Deutschkenntnisse verfügen. Dieses Kriterium wird durch Selbsteinschätzung der Kandidaten festgestellt. 
höchste abgeschlossene Bildung \& Land der Schulbildung:

Die Testpersonen müssen über einen bestimmten innereuropäischen Bildungsgrad verfügen. Dafür wird der Abschluss einer höher bildenden Schule durch Matura oder gleichwertigem Abschluss vorausgesetzt. Dieses Kriterium stellt sicher, dass die Teilnehmenden über eine möglichst homogene Vorbildung sowie über ein ähnliches kulturspezifisches Wissen verfügen. Kulturbedingte Unterschiede können sich beispielsweise durch eine andere Leserichtung, Vertrautheit mit unterschiedlichen Zeichennormen oder Ähnlichem auf das Lesen von Karten auswirken.

\section{Computerverwendung:}

Damit die Testergebnisse nicht durch Probleme der Computer- und Internetbedienung beeinflusst werden, wird eine gewisse Vertrautheit im Umgang vorausgesetzt. Die Testpersonen müssen sich sicher im Umgang mit Computer und Internet fühlen. Auch dieses wird durch Selbsteinschätzung der Testpersonen festgestellt.

\section{Wohnort, Arbeitsort, Geographisches Wissen:}

Der Usability-Test zur Benützung von KIT-Karten wird anhand von zwei Beispielstädten durchgeführt. Wichtig ist, dass alle Testteilnehmenden etwa über ähnliche Kenntnisse der beiden Städte verfügen. Wie bereits in Kapitel 4.1.1.2 beschrieben, soll es sich dabei um eine Stadt handeln, zu der die Teilnehmenden einen persönlichen Bezug haben, und eine andere, die dahingehend weiters unbekannt ist.

Da Wien als bekannte Stadt definiert wird, muss der Wohn- oder Arbeitsort der Testpersonen in Wien gelegen sein. Kopenhagen, als Beispiel einer unbekannten Stadt, soll von keiner der Testpersonen als Stadt zum Wohnen oder Arbeiten genannt werden. Auch wenn kein persönlicher Bezug zu Kopenhagen aufgrund dieser zwei Kriterien besteht muss dennoch ausgeschlossen werden, dass kein Hintergrundwissen vorhanden ist. Dieses wird anhand einer zu lösenden Aufgabe festgestellt in der fünf Umrisse von europäischen Städten durch ihre administrativen Bezirksgrenzen präsentiert werden. Diese müssen von allen Testpersonen mit den entsprechenden Namen versehen werden. Es wird angenommen, dass Personen, die die Abbildung nicht zuordnen können daher keine Kenntnisse über den administrativen Umriss von Kopenhagen haben und somit auch über keine weiteren Kenntnisse über für den Usability-Test relevanten räumlichen Ausprägungen verfügen. Um ein Minimum an Vorwissen über Wien festzustellen, muss Wien anhand der administrativen Grenzen von allen Testteilnehmenden erkannt werden. Jene Personen, die die Umrisse von Kopenhagen erkennen bzw. die Umrisse von Wien nicht erkennen, werden von der Teilnahme am Usability-Test ausgeschlossen. Das Erkennen bzw. Nicht-Erkennen der anderen Städte in dieser Aufgabe ist für die Auswahl der Testpersonen nicht relevant.

\section{b) Differenzierung zwischen Neulingen \& Experten}

Die Unterscheidung zwischen ExpertenInnen und Neulingen erfolgt anhand verschiedener Kriterien. Diese beziehen sich nicht auf spezifisches thematisches Wissen über die dargestellten Karteninhalte, sondern auf die Vertrautheit im Umgang mit thematischen Karten.

Neulinge zeichnen sich durch wenig Routine im Umgang mit Karten und einer geringen Vertrautheit in ihrer Verwendung aus. Neulinge sind Personen, die keine kartographische Ausbildung absolviert haben und weder privat noch beruflich in regelmäßigem Kontakt mit der Verwendung von thematischen Karten stehen. 
Als ExpertInnen werden jene Kartennutzenden kategorisiert, die über entsprechendes Hintergrundwissen und Erfahrungen im Umgang mit Karten verfügen. Sie verfügen über einen Abschluss eines kartographischen Studiums oder haben einen Studiengang absolviert, in dem die Verwendung von Karten essentiell ist. Weiters zählen jene Personen zur Gruppe der ExpertInnen, die aufgrund privater Interessen oder spezieller studien- oder berufsbezogenen Aufgabenstellungen über Vertrautheit in der Verwendung von thematischen Karten verfügen.

Bei dieser Differenzierung ist jedoch zu bedenken, dass unter den ExpertInnen aufgrund der Vertrautheit mit der Verwendung von Karten, bereits auch einige Karteninhalte bekannt sein können. Es kann daher der Fall auftreten, dass die ExpertInnen auch über einen Vorteil bei den inhaltlichen Themen verfügen, da sie bspw. schon öfter mit Karten zu Einwohnerdichte in Wien zu tun hatten bzw. aufgrund ihres allgemeinen Interesses an räumlichen Thematiken über Vorwissen verfügen.

\subsubsection{REKRUTIERUNG}

Die Auswahl der Testpersonen erfolgt über einen Eignungstest, der unter Kapitel 4.2.2 aufgestellten Kriterien der Testpersonen prüft. Neben dem Erfüllen dieser Kriterien sind weiters bestimmte Quoten zu beachten. Es ist auf einen ausgeglichenen Anteil von männlichen und weiblichen Testpersonen zu achten, sowie auf die Teilnahme von Neulinge und ExpertInnen zu jeweils fünfzig Prozent. Der Eignungstest wird solange durchgeführt, bis die entsprechende Zahl von qualifizierten Teilnehmenden am Usability-Test erreicht wurde. In Anhang C findet sich der dazu konzipierte Online-Test samt kategorisierter Antwortmöglichkeiten.

\subsection{DER USABILITY-TEST}

Jene Personen, die die in Kapitel 4.2.2 aufgestellten Kriterien erfüllen, sind qualifiziert am UsabilityTest teilzunehmen. Dieser besteht aus drei Teilen: Dem Vortest, dem Anwendungstest und dem Nachtest. Im Vortest werden allgemeine Fragen zur Verwendung von KIT-Karten gestellt. Gefolgt wird dieser vom Anwendungstest, der das eigentliche Herzstück des Usability-Tests darstellt. Die Testpersonen werden aufgefordert, sich mit einer KIT-Karte zu beschäftigen und müssen anschließend Fragen zum Inhalt beantworten. Abschließend folgt der Nachtest, in dem die qualitative Einschätzung der Testpersonen abgefragt wird. Abbildung 4-5 zeigt eine Skizze über den Aufbau des Usability-Tests. Sie stellt die Aufgabenstellung an die Teilnehmen dar und setzt diese in Verbindung mit den zu testenden Hypothesen.

Nachdem in Kapitel 3 die grundlegende methodische Herangehensweise bereits festgelegt wurde, wird in diesem Kapitel der detaillierte Aufbau des Usability-Tests mit seinen Komponenten erklärt. Im folgenden Kapitel wird beschrieben nach welchen Methoden die aus Kapitel 2 abgeleiteten Hypothesen in den Usability-Test integriert und mit den Aufgabestellungen der Testpersonen verknüpft werden. 


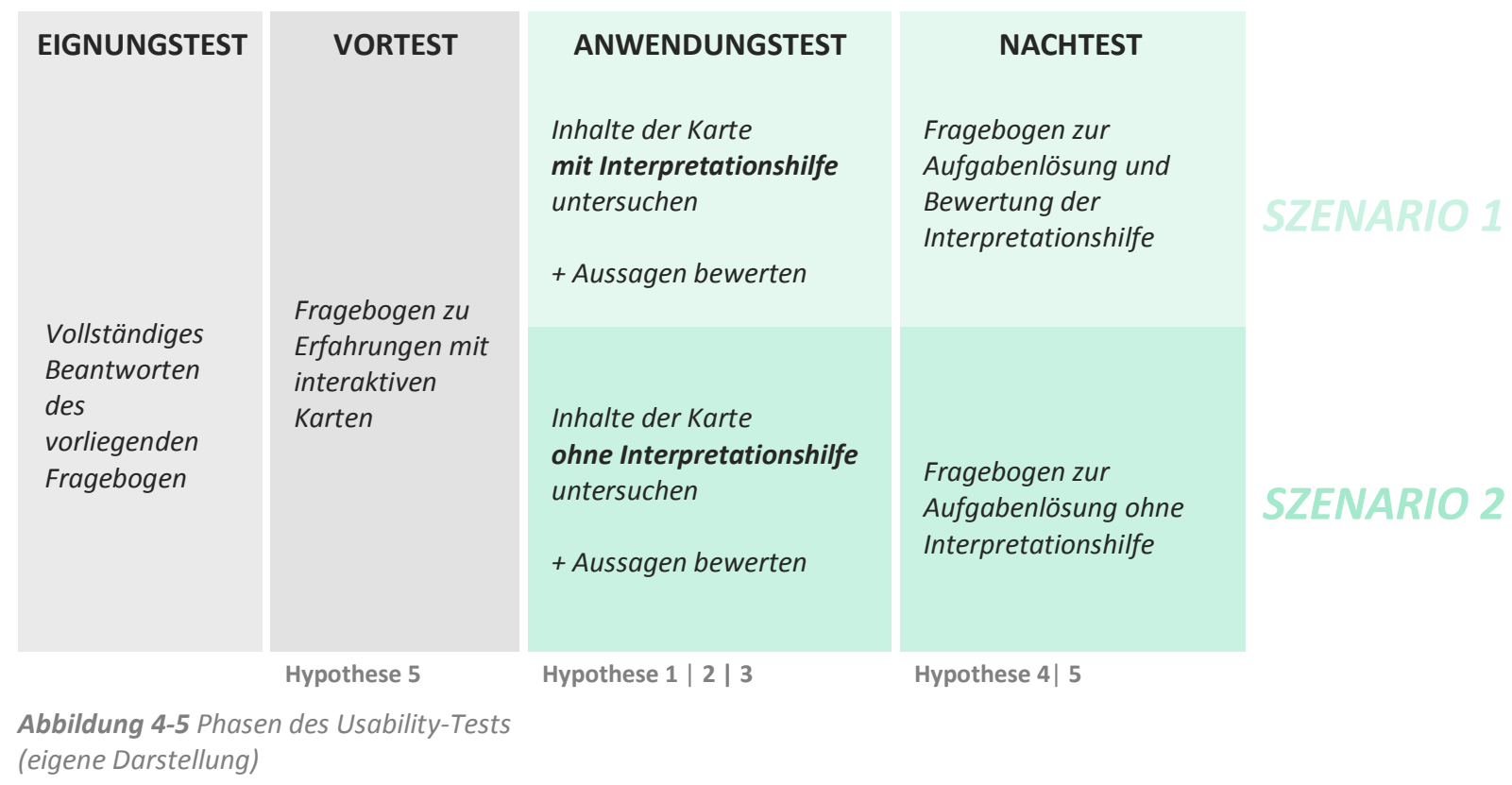

\subsubsection{VORTEST}

Im Eignungstest wurden persönliche Angaben zu Alter, Geschlecht und abgeschlossener Bildung abgefragt. Neben diesen soziodemographischen Charakteristiken werden auch bereits spezifische Attribute wie Vorwissen über die Verwendung von Karten erhoben. Im Vortest werden nun jene Bereiche abgedeckt, die weiters die Testergebnisse beeinflussen können. Dabei soll für beide Testgruppen in Erfahrung gebracht werden, wie vertraut sie im Umgang mit KIT-Karten im speziellen sind und wie sie deren Verwendung beurteilen.

Der Vortest dient nicht nur dazu Informationen über die Teilnehmenden zu sammeln, die für Interpretation der Testergebnisse relevant sind $[\mathrm{S} 1: 1,2 ; \mathrm{S} 2 \text { : Frage 1, 2, 3] }]^{3}$, sondern auch dazu, bereits erste Hinweise zur Beantwortung von Hypothesen [S1: Fragen 3 in Kombination mit Frage 10 für Hypothese 5] zu sammeln. In Anhang D findet sich der ausgearbeitete Fragebogen in dem die Fragen des Vortests enthalten sind.

\subsubsection{ANWENDUNGSTEST}

Der Anwendungstest ist das eigentliche Kernstück des Tests, bei dem zwei Methoden zum Wissensgewinn angewendet werden. Durch den Anwendungstest werden Hypothese 1, 2 und 3 beantwortet. Zum Einen geschieht das durch das konkrete Stellen von Wissensfragen an die Testpersonen, zum Anderen durch Beobachtung der Testpersonen bei der Benützung der Karte. Im Folgenden wird nun beschrieben, wie die jeweiligen Hypothesen durch die im Anwendungstest verwendeten Methoden bearbeitet werden.

\footnotetext{
${ }^{3}$ Kürzel für entsprechende Frage im Fragebogen

S1: Frage aus Fragebogen für Szenario 1; S2: Frage aus Fragebogen für Szenario 2.
} 


\title{
4.3.2.1. Wissensfragen
}

Hypothese 1 und Hypothese 2 werden durch einen Anwendungstest und seinen Wissensfragen getestet. Bei Wissensfragen geht es um Fragen, die objektiv mit richtig oder falsch zu beantworten sind. In Anhang D kann die genaue Aufgabenstellung beider Testgruppen nachgelesen werden.

\section{Hypothese 1}

Die Verwendung einer Interpretationshilfe führt zur Interpretation von bestimmten Inhalten.

Hypothese 1 testet die Aufmerksamkeitslenkung und -ablenkung beim Lesen der Inhalte innerhalb einer Kartenabbildung. Sie gibt Aufschluss darüber, welche Inhalte von den Nutzenden bei Verwendung der Interpretationshilfe wahrgenommen werden. Diese Hypothese bezieht sich ausschließlich auf Beobachtung innerhalb Szenario 1.

Folgende Unterhypothesen dienen zur Evaluierung dieser Hypothese:

\author{
Unterhypothese 1.1 \\ Nur die von der Interpretationshilfe korrekt genannten Zusammenhänge werden von \\ den Testpersonen richtig erkannt.
}

Mit dieser Unterhypothese wird vermutet, dass sich die Testteilnehmenden stark auf die inhaltliche Beschreibung der Interpretationshilfe zur jeweiligen Karte verlassen, ohne ihre Richtigkeit, Relevanz und weiter Inhalte selbstständig zu prüfen.

Um die Hypothese zu testen, werden Aussagen zu inhaltlichen Zusammenhängen der in der KIT-Karte dargestellten Thematik aufgestellt. Die Testpersonen müssen diese anschließend ohne Karte mit richtig oder falsch bewertet. Bei den Aussagen besteht ein unterschiedlicher Bezug zwischen den von Interpretationshilfe beschriebenen inhaltlichen Zusammenhängen und den tatsächlich in der Karte dargestellten Inhalten [S1,2: Frage 5].

Folgende Fälle werden getestet:

1.1.a) Aussage zu inhaltlichem Zusammenhang, der von der Interpretationshilfe angesprochen und in der Karte abgebildet ist.

1.1.b) Aussage zu inhaltlichem Zusammenhang, der von der Interpretationshilfe nicht angesprochen, aber in der Karte eindeutig sichtbar ist.

1.1.c) Aussage zu inhaltlichem Zusammenhang, der auf der Karte eindeutig zu erkennen ist, jedoch von der Interpretationshilfe falsch beschrieben wird.

In Anhang B findet sich die Auflistung, welche Aussagen nach welchem Fall erstellt wurden.

Zur Bewertung der Unterhypothese werden die Antworten dieser Aussagenbewertung der Teilnehmenden in Szenario 1 untersucht. 


\section{Unterhypothese 1.2}

Interpretationshilfe beeinflusst vor allem die Informationsaufnahme der Neulinge.

Weiters sind Zusammenhänge zu erwarten, die sich aufgrund der unterschiedlichen Testgruppen ergeben. Es wird erwartet, dass Neulinge aufgrund ihrer geringeren Erfahrung im Kartenlesen bei der Aussagenbewertung eher die in der Interpretationshilfe enthaltenen Informationen widergeben als ExpertInnen (z. B. Falschaussagen nach 1.1.c).

Zur Bewertung der Unterhypothese werden die aus der Aussagenbewertung gewonnenen Ergebnisse den entsprechenden Testgruppen zugeordnet.

\section{Hypothese 2}

Durch die Verwendung einer Interpretationshilfe werden die dargestellten Inhalte häufiger korrekt interpretiert.

Hypothese 2 testet das Verständnis über die wesentlichen Inhalte der Karte. Sie gibt Aufschluss darüber, ob diese von den Teilnehmenden auch ohne Interpretationshilfe erkannt werden. Sie bezieht sich auf den Vergleich der Ergebnisse [S1,2: Frage 5] aus Szenario 1 und Szenario 2.

Die Bewertung der Hypothese erfolgt anhand des Vergleichs der Ergebnisse der Aussagenbewertung zwischen Szenario 1 und 2. Für Hypothese 2 werden weiters zwei weitere Aspekte untersucht:

\section{Unterhypothese 2.1}

Die Interpretationshilfe verbessert vor allem die Interpretation der Neulinge.

Die Unterhypothese behauptet, dass Neulinge, da sie weniger vertraut sind im Kartenlesen, mehr von Hilfen zur Interpretation von Karteninhalten profitieren als ExpertInnen. Es wird erwartet, dass die Anzahl der richtigen Antworten der Neulinge im Vergleich zu den Expertinnen bei der Verwendung der Interpretationshilfe höher steigt.

Zur Bewertung der Unterhypothese werden die Aussagenbewertungen der jeweiligen Testgruppen zwischen Szenario 1 und Szenario 2 verglichen.

\section{Unterhypothese $\mathbf{2 . 2}$}

Der positive Einfluss der Interpretationshilfe tritt vor allem bei unbekannten Orten auf.

Im Kontext mit bekannten und unbekannten Orten wird angenommen, dass die Interpretationshilfe die Nutzenden vor allem bei der Interpretation von Zusammenhängen in Gebieten, die den Testpersonen unbekannt sind, hilfreich ist.

Zur Bewertung der Unterhypothese werden die Aussagenbewertungen hinsichtlich der jeweiligen Städte zwischen Szenario 1 und Szenario 2 verglichen. 


\subsubsection{Beobachten}

Während die Testpersonen mit der Aufgabenlösung beschäftigt sind, werden die Aktionen der Testpersonen vom Testleitenden beobachtet und aufgezeichnet. Die Ergebnisse geben Aufschluss über Hypothese 3.

\section{Hypothese 3}

Die Verwendung einer Interpretationshilfe führt zu einem verminderten Explorieren in der Karte.

Hypothese 3 testet den Einfluss der Interpretationshilfe auf die Interaktion der Testpersonen mit der KIT-Karte. Sie gibt Aufschluss darüber, wie intensiv sich die Testpersonen mit der Anwendung beschäftigen und welchen Einfluss die Interpretationshilfe auf die Benützung der Karte hat.

Zur Beantwortung dieser Hypothese werden das Klickverhalten während dem Erkunden der Karte und die dafür aufgebrachte Zeit [S1, S2: 4] zwischen Szenario 1 und Szenario 2 vergleichen.

\section{Unterhypothese 3.1}

Es werden wenig neue Bereiche neben den von der Interpretationshilfe angesprochenen Bereichen aufgerufen.

Es wird erwartet, dass sich bei Vorhandensein einer Interpretationshilfe die Teilnehmenden hauptsächlich entlang des von der Interpretationshilfe vorgebenden Pfades bewegen. Nicht angesprochene Inhalte werden seltener selbstständig besucht.

Um Aussagen zu dieser Unterhypothese zu sammeln, wird das Klickverhalten während der Aufgabenlösung [S1: 4] der Teilnehmenden beobachtet.

Um die Unterhypothese zu beantworten, werden von der Interpretationshilfe bei ihrer Führung durch die Karte bewusst wichtige Kartenkombinationen ausgelassen. Bewertet wird die Unterhypothese dadurch, ob die Teilnehmenden in Szenario 1 während oder nach dem Ende der von der Interpretationshilfe vorgegeben Führung selbstständig die zusätzlichen Inhalte aufrufen.

\section{Unterhypothese 3.2}

Ist eine Interpretationshilfe vorhanden verbringen die Testpersonen weniger Zeit mit der Verwendung der Anwendung.

Die Unterhypothese vermutet, dass durch die gezielte und kompakte Leitung der Interpretationshilfe durch die KIT-Karte die Testpersonen in Szenario 1 weniger Zeit mit der Verwendung der Anwendung verbringen. Dafür wird gemessen, wie lange sich die Testpersonen mit der Karte beschäftigen.

Zur Bewertung der Unterhypothese wird die für die Kartenerkundung aufgebrachte Zeit [S1, S2: 4] untersucht. Es werden daraufhin Vergleiche angestellt, ob die zu Verfügung gestellte Zeit in Szenario 1 und Szenario 2 gleich ausgenutzt wird. 


\section{Unterhypothese 3.3}

Die Interpretationshilfe vermindert vor allem das Explorieren der Neulinge.

Durch die Führung der Interpretationshilfe in der KIT-Karte wird vor allem ein Einfluss auf die Testgruppe der Neulinge erwartet. Neulinge sind weniger vertraut im Lesen von Karten und könnten sich daher eher von den Inhalten der Interpretationshilfe leiten lassen. Es wird vermutet, dass sie im Vergleich zu ExpertInnen weniger zusätzliche Bereiche neben den von der Interpretationshilfe präsentierten Inhalten aufrufen. Weiters besteht die Annahme, dass sich die verbrachte Zeit durch Einsatz der Interpretationshilfe deutlicher bei den Neulingen als bei den ExpertInnen verkürzt.

Bewertet wird die Unterhypothese durch den Vergleich der aufgerufenen Bereiche und der verbrachten Zeit in Bezug auf die Szenarien und Testgruppen.

\subsubsection{NACHTEST}

Im Nachtest wird die subjektive Meinung über die Testanwendung durch Einstellungsfragen erhoben. Er dient dazu nachvollziehen zu können, warum es den Testpersonen beim Lösen der Aufgaben [S2,1: 5] wie ergangen ist. Dazu enthält der Nachtest Fragen zum allgemeinen Verlauf der Aufgabenlösung [S1, S2: 6, 7, 8], dessen Antworten Erkenntnisse zu Hypothesen 4 und 5 liefern. Für Szenario 1 werden die Teilnehmenden weiters gebeten, ihre Verwendung zu bewerten [S1: 9, 10, 11]. Weiters sind allgemeine Fragen zu technische Schwierigkeiten oder sonstige besondere Umstände bei der Bearbeitung der Testaufgeben enthalten [S1:12, 13, 14; S2:9, 10, 11]. In Anhang D befinden sich die im Nachtest gestellten Fragen.

Hypothese 4 Die Testpersonen fühlen sich von der Interpretationshilfe bevormundet.

Hypothese 4 testet wie die Testpersonen auf die in der Interpretationshilfe enthaltenen Inhalte reagieren. Sie stellt fest, ob die Inhalte der Interpretationshilfe von den Testpersonen negativ bewertet werden. Für die differenzierte Beantwortung der Hypothese werden folgender Zusammenhänge erwartet:

\section{Unterhypothese 4.1}

Die Interpretationshilfe wird hauptsächlich von ExpertInnen negativ beurteilt.

Es wird angenommen, dass vor allem Teilnehmende aus der Testgruppe der ExpertInnen die Inhalte der Interpretationshilfe negativ beurteilen. Im Gegensatz zu den Neulingen, haben diese eine stärkere eigene Meinung über die Inhalte der Karte und sind eher nicht mit den Inhalten der Interpretationshilfe einverstanden.

Getestet wird die Unterhypothese anhand von entsprechenden Fragen im Nachtest [S1: $9,10,11]$ zu den Erfahrungen mit der Interpretationshilfe. 


\section{Hypothese 5}

Die Interpretationshilfe wird von den Testpersonen als hilfreich und unterstützend wahrgenommen.

Wie Hypothese 4 testet auch Hypothese 5 die Reaktion der Testpersonen auf die Inhalte der Interpretationshilfe. Diese Hypothese stellt fest, ob die Interpretationshilfe von den Testpersonen subjektiv als positiv bewertet wird. Getestet wird dieses anhand durch Fragestellungen im Vortest und Nachtest. [S1: Vergleich von Frage 3 mit Frage 12 in Kombination mit Frage 9,10,11]

Die Beantwortung der Hypothese erfolgt hinsichtlich zwei Unterhypothesen:

\section{Unterhypothese 5.1}

Die Interpretationshilfe wird hauptsächlich von Neulingen positiv beurteilt.

Zur Bewertung der Unterhypothese werden die Meinungen über die Interpretationshilfe der jeweiligen Testgruppen zugeordnet und verglichen.

\section{Unterhypothese 5.2}

Die Interpretationshilfe wird vor allem bei unbekannten Orten als Hilfe empfunden.

Zur Bewertung dieser Unterhypothese werden die Meinungen über die Interpretationshilfe hinsichtlich Hilfestellungen zu bekannten und unbekannten Orten verglichen. Weiters wird das subjektive Schwierigkeitsempfinden der Aufgabenlösung mit dem Vorwissen über den Ort in Bezug gesetzt.[S1: 7, 10 in Kombination mit Frage 5]

\subsubsection{TESTABLAUF}

Im vorangegangen Punkt wurde erklärt, welche Elemente ausschlaggebend für den Testaufbau sind. Diese werden nun zu einem flüssigen Testablauf zusammengestellt, der alle diese Elemente abdeckt. Die Testaufgaben sind so gestaltet, dass sie in angemessener Zeit bearbeitbar, aber dennoch entsprechend komplex sind, um Aussagen über den Einfluss der Interpretationshilfe treffen zu können. Um für alle Testpersonen einen gleichen Testablauf zu gewährleisten, wird eine Struktur aus Anweisungen und Aufgabenstellungen festgelegt. Die einzelnen Abschnitte werden nun beschrieben.

\subsubsection{Vorbereitungen}

Nachdem die Teilnehmenden auf Erfüllen der zur Testteilnahme erforderlichen Voraussetzung geprüft wurden, wird für jede Testperson einzeln ein Testtermin und -ort vereinbart. Wie bereits in Kapitel 3.4.1 beschrieben, kann der Test bezüglich des Ortes sehr flexibel durchgeführt werden.

\subsubsection{Vortest}

Vor Testbeginn bekommt jeder Testteilnehmende die Testmodalitäten in mündlicher Form vorgetragen. Diese werden für jeden Teilnehmenden abgelesen um sicherzustellen, dass jede Testperson gleich informiert ist. Anschließend werden den Teilnehmenden die Fragen des Vortests präsentiert. Hier ist die Rolle des Testleitenden sehr aktiv. Durch gemeinsames Beantworten des Fragebogens soll 
die Möglichkeit bestehen, tiefergehende Antworten zu erlangen als wenn die Testpersonen den Fragebogen selbstständig schriftlich ausfüllen. Der Vortest enthält eine Kombination aus MultipleChoice-Fragen, Analogskalen und offenen Fragen. Der Vortest ist für beide Szenarien ident.

\subsubsection{Anwendungstest}

Danach folgt der Hauptteil des Usability-Tests, der die Benützung der KIT-Karten-Anwendung beinhaltet. Die Testpersonen erhalten eine schriftlich ausgehändigte Aufforderung, sich innerhalb einer begrenzten Zeit nach bestimmten Gesichtspunkten mit den Inhalten der KIT-Karte auseinanderzusetzen. Den Testpersonen steht es frei sich zu ihren Beobachtungen Notizen zu machen. Diese fließen nicht in den Testausgang ein und werden nicht bewertet. Die Aufgabe ist von den Testpersonen selbstständig zu lösen. Grundsätzlich bekommen beide Testgruppen die gleiche Aufgabenstellung im Anwendungstest, deren Ausführung sich jedoch aufgrund der zwei definierten Szenarien unterscheidet. Die Anweisung der beiden Testgruppen unterscheidet sich nur dadurch, dass die Testpersonen des Szenario 1 aufgefordert werden, der Interpretationshilfe zu folgen. In Szenario 2 entfällt diese.

Rubin, Chisnell und Spool (2008) weisen darauf hin, dass direktes Eingeben von Daten in den Computer Tastengeräusche erzeugt, die einschüchternd auf die Testpersonen wirken können. Dementsprechenden werden die Ergebnisse und Beobachtungen vom Testleitenden zunächst auf Papier festgehalten und erst nachträglich digital in einer Excel-Datei verarbeitet.

\subsubsection{Nachtest}

Der Nachtest besteht ähnlich dem Vortest wieder aus einer Kombination von Multiple-ChoiceFragen, Analogskalen und offenen Fragen. Die Rolle des Testleitenden wird aktiv, indem er die Fragen gemeinsam mit den Testpersonen durchgeht. Der Nachtest ist jeweils auf das entsprechende Szenarium abgestimmt. Er unterscheidet sich in jenen Bereichen, die sich konkret auf die Bewertung der Interpretationshilfe beziehen.

\subsubsection{Auswertung}

In Anhang $D$ finden sich die Fragebögen, die im Test zum Einsatz kommen. In diesen ist die unterschiedliche Farbgebung Schwarz und Blau zu erkennen. Sie gibt Hinweise über die Auswertung der von den Testteilnehmenden gegeben Antworten.

Die in schwarz verfassten Antwortmöglichkeiten legen den Befragten standarisierte Multiple-ChoiceMöglichkeiten vor. Diese werden direkt an die Teilnehmenden vorgetragen, die sich auf eine oder mehrere der angebotenen Kategorien festlegen müssen.

Die in blau verfassten Antwortmöglichkeiten verlangen eine eigene Einschätzung der Befragten. Der Testleitende gibt bei dieser Art von Frage keine Antwortmöglichkeiten vor, sondern fordert die Teilnehmenden zu einer freien Antwort auf. Entspricht die Antwort einer bereits vom Testleitenden erwarteten Kategorien, wird diese noch während dem Test dieser zugeordnet. Weiters wird durch das blaue "[Warum]", das beinahe im Anschluss jeder Frage gestellt wird, eine halboffene Fragestellung suggeriert. Diese soll es Testpersonen ermöglichen auch bei Multiple-Choice-Fragen mehr über die Hintergründe der Antwort preiszugeben. 
Nach Durchführen der Tests werden die Ergebnisse in eine Excel-Datei übertragen, in der nach Abschluss aller Tests die Ergebnisse ausgewertet werden. Die gewonnen Ergebnisse werden anschließend in Kapitel 4.4. beschrieben.

\subsection{ERGEBNISSE}

Durch die empirischen Methoden, die im Usability-Test eingesetzt wurden, können die Hypothesen und Unterhypothesen nach der in Kapitel 3.2.3 beschriebenen Methode untersucht und in weitere Folge die Forschungsfrage beantwortet werden.

Dieses Kapitel beinhaltet eine Beschreibung der am Test teilgenommen Personen sowie der der zentralen Fragestellungen des Usability-Tests, aus der viele Daten abgeleitet werden. Je Hypothese wird dargestellt, wie diese aufgrund des Testausgangs zu bewerten ist. Abschließend werden die gewonnenen Ergebnisse hinsichtlich ihrer Einschränkungen und Signifikanz beschrieben.

\subsubsection{TESTBESCHREIBUNG}

Für diesen Usability-Test wurde die unter Kapitel 4.2.1 definierte notwendige Anzahl an Testpersonen mit den entsprechenden Voraussetzungen gefunden. Der Test konnte daher im Jänner 2015 innerhalb von zwei Wochen durchgeführt werden. Die Testgruppe besteht aus 10 weiblichen und 10 männlichen Personen im Alter zwischen 24 und 28 Jahren.

Nach den in Kapitel 4.2.2. festgelegten Kriterien, wird zwischen "Neulingen" und "ExpertInnen" unterscheiden. Die Gruppe der Neulinge setzt sich aus Studierenden der Studienrichtungen Physik, medizinischer Informatik und Journalismus \& Medienwissenschaft zusammen. Weiters nahmen ein Kellner, ein Elektronik-Entwickler und ein Promotor sowie eine Business Analystin und eine Programmiererin am Test teil. Die Gruppe der ExpertInnen setzt sich aus fünf RaumplanerInnen bzw. Raumplanungsstudierenden zusammen sowie Studierenden der Studienrichtungen Geographie und Architektur. Vervollständigt wurde die Testgruppe durch eine Immobilien Consultin und einen Informatiker.

Durchgeführt wurden die Tests in den Räumen der TU Wien, bei den Testteilnehmenden sowie bei dem Testleitenden zu Hause und in Cafés. Die Dauer der Tests variierte je nach Ausführlichkeit der Antworten sowie nach aufgewendeter Zeit zur Verwendung der Anwendung. Die Spanne reichte dabei von 16 bis 74 Minuten, wobei in den meisten Fällen eine ungefähre Zeit von 30 Minuten eingehalten wurde.

\subsubsection{AUSGANGSLAGE}

In der Gruppe der Neulinge gaben fast alle Testpersonen an, noch nie KIT-Karten verwendet zu haben. Sie seien weder beruflich, studienbezogen noch privat mit diesen konfrontiert. Allein drei Testpersonen geben an, KIT-Karten schon einmal verwendet zu haben. Als Zusammenhang werden Recherchen im Bereich Datenjournalismus sowie Informationssuche zu aktuellen Themen (z.B. Wahlergebnisse) genannt. In der Gruppe der ExpertInnen, die laut Definition bereits Erfahrungen im Um- 
gang mit thematischen Karten haben, verwenden zumindest alle "hin und wieder" digitale Karten, die den Eigenschaften von KIT-Karten entsprechen. Diese wurden hauptsächlich im Zuge von studienbezogenen Recherchen und zur Informationssuche zu aktuellen Themen verwendet. Einige der ExpertInnen gaben an, sich selbst schon mit der Herstellung solchen Karte beschäftigt zu haben. Abbildung 4-6 zeigt eine Übersicht zur Häufigkeit der Verwendung von KIT-Karten.

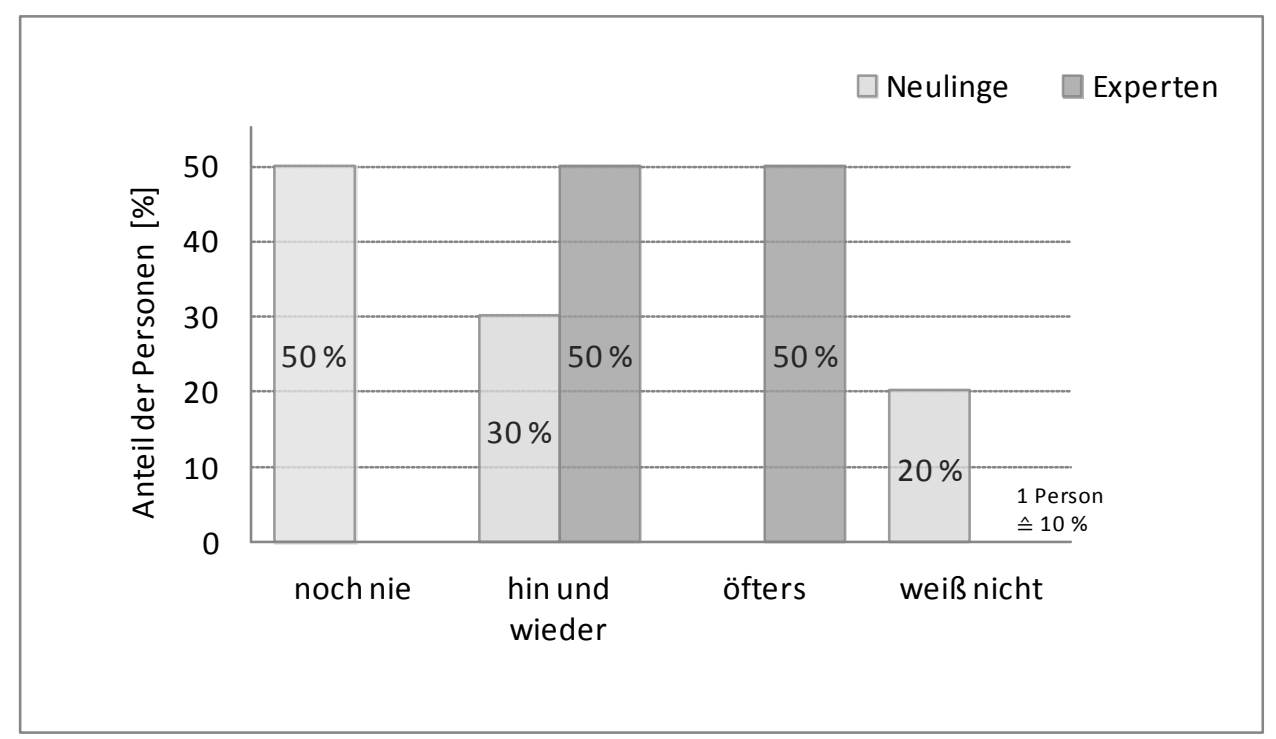

Abbildung 4-6 Häufigkeiten zur Verwendung von KIT-Karten von Neulingen und ExpertInnen (eigene Darstellung)

\section{a) Bewertung der KIT-Karte durch die Neulinge}

Bei der Verwendung dieser Karten haben die Testpersonen unterschiedliche Erfahrungen gemacht. Jene drei Teilnehmenden der Neuling-Gruppe bewerten die Benützung von KIT-Karten durchwegs positiv. Dabei werden diese Karten von den Testpersonen spontan mit Eigenschaften wie "übersichtlich" (TP 844), "hilfreich, einfach" (TP 82) beschrieben. Weiters wird die Verwendung von KIT-Karten als "lustig" beschrieben und positiv hervorgehoben, dass man "viel umstellen und anpassen" (TP 77) kann.

Abbildung 4-7 zeigt, wie die Bewertung von KIT -Karten hinsichtlich bestimmter Punkte von den Neulingen eingeschätzt wird. Zur allgemeinen Verwendung von KIT-Karten wird beispielsweise genannt, dass "wenn man zu viel tun kann, ist die Verwendung mühsam" (TP 77). Die Testperson setzt diese Aussage in den Kontext von KIT-Karten, die auf Nachrichtenwebsites veröffentlicht werden. Sie weist darauf hin, dass man auf diesen Seiten nicht viel Zeit verbringe und wenn man dann "Iange damit beschäftigt ist in der Karte Dinge umstellen, ist das die Arbeit oft nicht wert" (TP 77). Es würden zu viele Informationen präsentiert, die zu einem "Information Overload" (TP 77) führen. Weiters wird die Bewertung des Zwecks der KIT-Karte als Spielerei von einem Neuling damit begründet, da damit "Leute eher angesprochen" (TP 82) werden.

Zusammenfassend werden die KIT-Karten jedoch als eine informative Methode bewertet, um konkrete Informationen durch einen ansprechenden spielerischen Zugang zu erfassen. Da aus der Grup-

\footnotetext{
${ }^{4}$ TP 84: Kürzel für Testperson, siehe Anhang $\mathrm{E}$
} 
pe der Neulinge nur Wenige Erfahrung mit der Verwendung von KIT-Karten haben, kann aufgrund der geringen Anzahl von Bewertungen nur vorsichtig auf die ganze Gruppe geschlossen werden.

Allgmeine Verwendung:

überfordernd

informativ

Erfassen von konkreten Informationen:

schwer

Zweck der Karte:

ansprechend

visualisierte Spielerei

Kommunikation

von Informationen

Abbildung 4-7 Einschätzung der Neulinge über die Verwendung von KIT-Karten

(eigene Darstellung)

\section{b) Bewertung der KIT-Karte durch die ExpertInnen}

Auch von den Teilnehmenden der ExpertInnen-Gruppe werden KIT-Karten mit positiven Eigenschaften verknüpft. "Übersichtlich" (TP 81) oder "gut verständlich" (TP 72) sind einige Eigenschaften, die in diesem Zusammenhang genannt werden. Der interaktive und explorative Charakter der KIT-Karten wird ebenfalls von den ExpertInnen erwähnt. Die Verwendung der KIT-Karte wird als "interessant" (TP 89) bewertet, da es durch ihre Eigenschaften leicht ist "Veränderung [im thematischen Inhalt] festzustellen" (TP 89) sowie" viel Infos zusammenzustellen"(TP 89).

Obwohl keiner der ExpertInnen eine rein negative Bewertung zur Verwendung von KIT-Karten abgeben hat, wurden die positiven Bewertung dennoch durch kritische Betrachtungen ergänzt. Beispielsweise wurde zur allgemeinen Verwendung angemerkt, dass die KIT-Karten "praktisch" (TP67) sind wenn man "neugierig ist und herum probieren möchte" (TP67). Bei der Verarbeitung von großen Datenmengen, die man auch als Quelle heranziehen möchte, seien Tabellen jedoch nützlicher (TP67). Weiters wird von einer Expertin vermutet, dass diese Karten für Leute mit raumplanerischen Hintergrund vermutlich "leichter zu verstehen sind als für Laien" (TP68).

Abbildung 4-8 zeigt die detaillierte Bewertung der verschiedenen Komponenten der KIT-Karte von den zehn ExpertInnen. In Bezug auf eine informative allgemeine Verwendung und dem leichten Erfassen von konkreten Informationen sind sich die ExpertInnen in ihrer Bewertung einig. Begründet wird dieses dadurch, dass "man auf den ersten Blick schon mal gut seht, was los ist" (TP67) sowie dass die Karte "sehr intuitiv" (TP 67) zu bedienen ist. Dennoch wird von den ExpertInnen darauf hingewiesen, dass das Ablesen von konkreten Daten "nicht so leicht wie bei einer Excel-Tabelle" (TP 70) ist und Vergleiche schwieriger anzustellen sind. Bei der Bewertung des Zwecks der Karte gibt es allerdings eine große Streuung. Hier ist kein allgemeiner Trend unten den ExpertInnen ablesbar. Einige Testpersonen sehen die Kommunikation von Informationen im Vordergrund, andere sehen den hauptsächlichen Zweck dieser Karten als Spielerei. Eine Testpersonen beschreibt die Verwendung der KIT-Karte hinsichtlich dieser Merkmale folgendermaßen: Ich spiele mich herum und versuche herauszufinden, was in der Karte abgebildet ist und welche Möglichkeiten sie bietet. [..] Ich merke mir dann 
viele Sachen nicht [..], nur die wichtigsten" (TP 69). Auch eine weitere Testperson meint dazu Ähnliches: "Wenn ich mir interaktive Sachen anschaue dann ist es so, dass ich ein bisschen herumspiele aber nachher nicht mehr sagen kann, was ich mir jetzt eigentlich genau angeschaut habe und was ich rausgefunden habe" (TP 78).

Allgmeine Verwendung:

überfordernd informativ

Erfassen von konkreten Informationen:

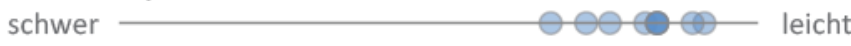

Zweck der Karte:

ansprechend

visualisierte Spielerei

Kommunikation

von Informationen

Abbildung 4-8 Einschätzung der ExpertInnen über die Verwendung von KIT-Karten

(eigene Darstellung)

Vergleicht man anhand der Analogskalen die durchschnittliche Bewertung der Neulinge mit der von den ExpertInnen wird die allgemeine Verwendung von KIT-Karten von den Neulingen als etwas überfordernder beurteilt. Von den Neulingen wird aber dennoch eher als von den ExpertInnen angegeben, dass das Erfassen von konkreten Informationen leicht ist. Generell werden KIT-Karten von den Neulingen eher als Spielerei gesehen, wohin gegen die Bewertung der ExpertInnen eher zur Kommunikation von Informationen tendiert. Wie von einigen ExpertInnen beschrieben, kann es sein, dass bei zu starkem "Spielen" Informationen nicht mehr wahrgenommen werden, sondern viel und eher willkürlich herum geklickt wird. Wird die KIT-Karte daher eher in Richtung Spielerei beurteilt, wird der Verbesserungsbedarf bestätigt. Wie bereits einige Testpersonen (TP 75, 68, 78) angemerkt haben, ist generell bei dieser Art von Bewertung zu bedenken, dass es stark auf die Karte ankommt, auf die sich diese Bewertung bezieht.

\subsubsection{AUSSAGENBEWERTUNG IN FRAGE 5}

Als zentrales Element zur Untersuchung der Hypothesen sind die Bewertungen und Ergebnisse zu Frage 5 des Usability-Tests. Frage 5 fordert die Testteilnehmenden beider Szenarien auf, neun Aussagen mit "richtig", "falsch" oder "weiß nicht" zu bewerten. Diese stehen dabei folgendem Muster in Zusammenhang:

Inhalt der Interpretationshilfe:

Zu bewertenden Aussagen:
"Im Bezug auf das Einkommensniveau der Wiener Bevölkerung weisen Bezirke wie 'Innere Stadt', 'Döbling', 'Währing' und 'Hietzing' neben einer hohen Dichte an Arztpraxen (über 30 ÄrztInnen pro 10.000 EW) auch ein hohes Nettoeinkommen auf."

In Wien ist die Anzahl der Arztpraxen in Gebieten mit hohem Einkommensniveau höher. 
Wie Kapitel 4.3.2.1 beschreibt, werden die Inhalte der Interpretationshilfe nach unterschiedlichen Fällen verfasst. Nicht alle textlichen Kommentare sind dadurch vollständig oder korrekt. Je nachdem wie vollständig und korrekt der Inhalt der Interpretationshilfe ist, kann es mehr oder weniger schwierig sein, die richtige Bewertung zu wählen. Im Zuge der Evaluierung der Hypothesen wird immer wieder auf diese Ergebnisse zurückgegriffen und versucht nach verschiedenen Gesichtspunkten Regelmäßigkeiten bei der Beantwortung abzuleiten.

\subsubsection{EVALUATION DER HYPOTHESEN}

Durch das Testverfahren wurden sowohl objektive Fragestellungen als auch subjektive Fragestellungen beantwortet. Die Quantitativ gemessenen Ergebnisse sowie die allgemeinen Einschätzungen der Testpersonen sind notwendig, um die aufgestellten Hypothesen zu evaluieren. Im folgendem werden nun jede Hypothese mit den erhobenen Daten beantwortet.

\subsubsection{Hypothese 1}

Die Verwendung einer Interpretationshilfe führt zur Interpretation von bestimmten Inhalten.

Der Vergleich der Ergebnisse aus Frage innerhalb Szenario 1 stellt fest, wie stark der Einfluss der Interpretationshilfe auf die Wahrnehmung der Karteninhalte ist.

Die Überprüfung von Hypothese 1 erfolgt durch ihre zwei Unterhypothesen:

\section{Unterhypothese 1.1}

Nur die von der Interpretationshilfe korrekt genannten Zusammenhänge werden von den Testpersonen richtig erkannt.

Tabelle 4-2 zeigt wie viele Aussagen von den Testpersonen in Szenario 1 richtig bewertet wurden. Insgesamt wurden $85 \%$ der Aussagen, die in der Interpretationshilfe nach den Kriterien aus 1.1a) erarbeitet wurden, von den Testpersonen richtig erkannt. $100 \%$ der Aussagen, die in der Interpretationshilfe nach den Kriterien aus 1.1b) erstellt wurden, konnten von den Testpersonen korrekt bewertet werden. Lediglich $25 \%$ der nach den Kriterien aus 1.1c) erstellten Falschaussagen wurden von den Testpersonen identifiziert und in der Bewertung richtig berücksichtigt.

\section{AUSSAGE RICHTIGE ANTWORTEN IN SZENARIO 1}

1.1a) Korrekt von Interpretationshilfe beschrieben

\begin{tabular}{|c|c|c|}
\hline $\mathbf{5 a}$ & $70 \%$ & \\
\hline $\mathbf{5 b}$ & $90 \%$ & \\
\hline $\mathbf{5 d}$ & $80 \%$ & $85 \%$ \\
\hline $\mathbf{5 e}$ & $100 \%$ & \\
\hline $\mathbf{5 g}$ & $90 \%$ & \\
\hline $\mathbf{5 i}$ & $80 \%$ & \\
\hline
\end{tabular}

Tabelle 4-2 Anteil der richtigen Bewertungen zu Frage 5 (eigene Darstellung) 
1.1b) Nicht von Interpretationshilfe beschrieben

$5 \mathrm{~h}$

$100 \%$

$100 \%$

1.1c) Falsch von Interpretationshilfe beschrieben

$5 c$

$40 \%$

$5 f$

$10 \%$

$25 \%$

1 Person $\triangleq 10 \%$

\section{a) Korrekt von Interpretationshilfe genannt (1.1a)}

$85 \%$ der Testpersonen konnten jene Aussagen richtig bewerten, die auch in der Interpretationshilfe korrekt beschrieben werden. Innerhalb dieser Gruppe variiert der Prozentsatz doch beachtlich von 70 bis $100 \%$. Für 5a) wurde beispielsweise angegeben, dass die Fragestellung zu diesem Zusammenhang unerwartet war, da sie sich die Verteilung der ÄrztInnen nicht im Zusammenhang mit dem bebauten Gebiet angesehen haben (TP 78).

Vergleicht man den Anteil richtiger Antworten von 85\% bei korrekter Interpretationshilfe (1.1a) mit dem Anteil richtiger Antworten von $50 \%$ bei unzureichender Interpretationshilfe (1.1b + 1.1.c) ist der Einfluss der Interpretationshilfe deutlich zu erkennen.

Es werden demnach mehr richtige Bewertungen durchgeführt, wenn die Inhalte der Interpretationshilfe korrekt sind.

\section{b) Nicht von Interpretationshilfe beschrieben (1.1b)}

$100 \%$ der Testpersonen konnten Aussage 5h) richtig beantworten. Obwohl in der textlichen Erklärung zum Karteninhalt keine relevante Erklärung zu den Darstellungen enthalten ist, konnten alle Testpersonen die entsprechende Aussage richtig bewerten. Unter vorsichtigen Annahmen könnte das Ergebnis daraus entstanden sein, dass es sich dabei um einen sehr logischen Zusammenhang handelt. Eine Testperson gibt an nach der generellen Annahme "dort wo viel Geld und viele Leute sind, gibt es viele Ärzte" (TP 77). Auch weitere Testpersonen erklären, sie haben die Karte "auch nach Logik" (TP 85) beantwortet und Erinnerungen und Notizen zur Karte seien nur "teils-teils" (TP 85) für die Lösung der Aufgaben herangezogen worden. Sie habe sich "bei Kopenhagen eher an die Karten erinnert, bei Wien eher mehr [nach] Logik" (TP 85) beantwortet.

Wird eine bestimmte Aussageebene in die Tour der Interpretationshilfe integriert, zu der jedoch erklärende Texte vorenthalten werden, können die Testpersonen die Darstellungen trotzdem korrekt interpretieren.

\section{c) Falsch von Interpretationshilfe beschrieben (1.1c)}

Nur 25 \% Personen konnten die Kommentare nach 1.1c) richtig beantworten. Zwei Aussagen wurden nach diesem Muster erstellt:

- Aussage 5c behauptet: "In Kopenhagen konzentrieren sich die Arztpraxen in Gebieten in denen das Einkommen der Bevölkerung hoch ist."

Auch der Inhalt der Interpretationshilfe bestätigt diese Annahme: "Im Zusammenhang mit 
dem Nettoeinkommen der Kopenhagener zeigt das grüne Balkendiagramm, dass, bis auf einen Ausreißer, die Anzahl der Arztpraxen in jenen Bezirken konzentriert ist, in denen das Einkommen der Bevölkerung hoch ist."

Abbildung 4-9 zeigt jedoch, dass in der KIT-Karte das genaue Gegenteil abbildet.

$60 \%$ der Testpersonen konnten die Bewertung der Aussage nicht richtig ausführen. Der Zusammenhang zwischen Einkommen und Verteilung der Arztpraxen in Kopenhagen könnte für viele Testpersonen unerwartet und unlogisch sein. Dieser Zusammenhang bildet eine Ausnahme, da die anderen Aussagen der KIT-Karte über einen positiven Zusammenhang miteinander verbunden sind. Hohe Einwohnerdichte, hohes Einkommensniveau und hohes Preisniveau bedeute eine hohe Anzahl von Arztpraxen. Es kann daher verlockend sein, logischen Denkmustern zu folgen und den Text der Interpretationshilfe nicht zu hinterfragen. Aussage $5 \mathrm{c}$ ) eignet sich daher besonders dafür, die Aufmerksamkeit gegenüber dem tatsächlichen Karteninhalt zu testen.

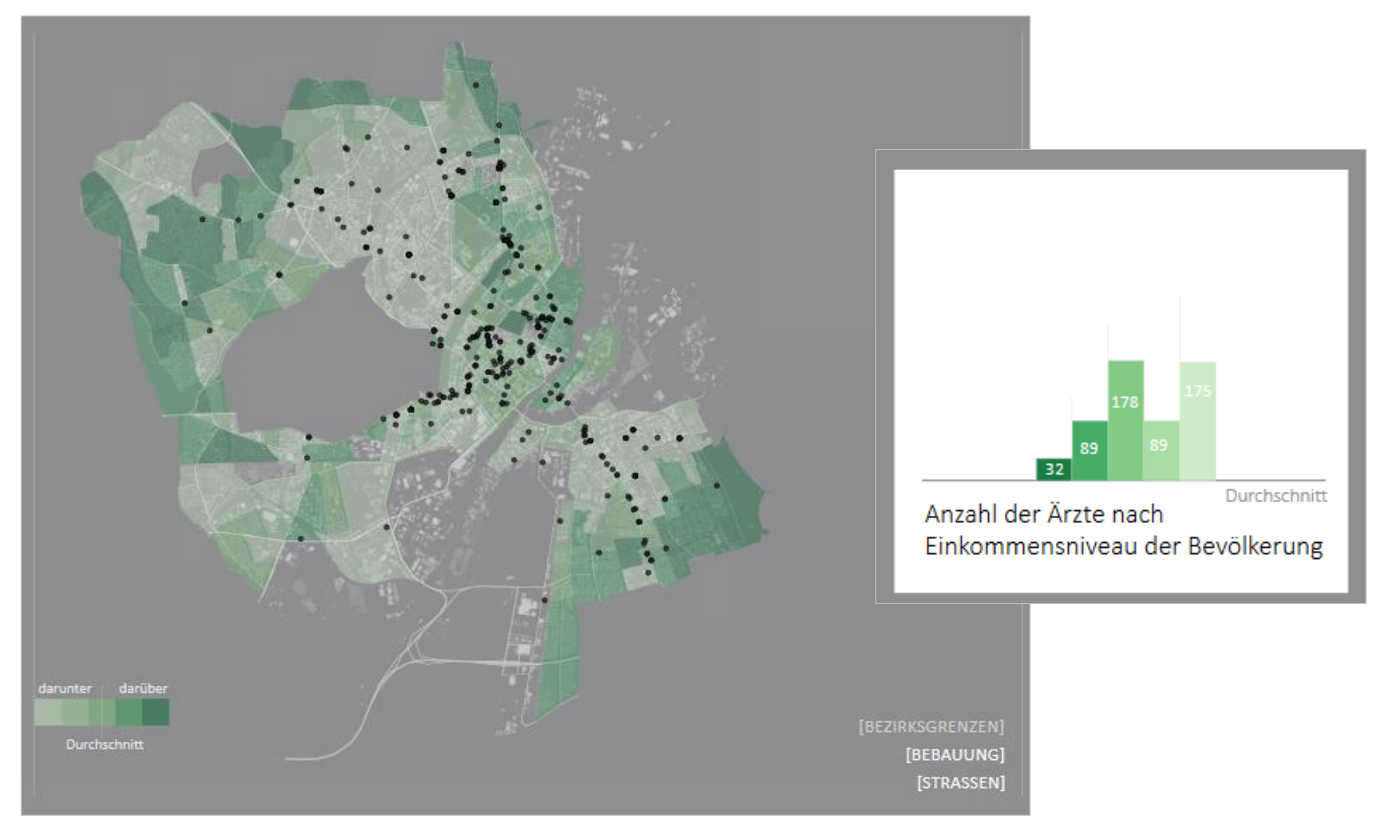

Abbildung 4-9 Verteilung der Arztpraxen in Kopenhagen in Bezug auf das Einkommensniveau der Bevölkerung (Screenshot KIT-Karte)

- Aussage 5f) behauptet folgendes zur Verteilung der Arztpraxen in Wien: "In Wien konzentrieren sich die Arztpraxen entlang von Straßen, die sich sternförmig vom geographischen Zentrum der Stadt ausbreiten."

Durch den Inhalt der Interpretationshilfe wird diese Aussage ebenfalls unterstützt: Auch in Wien ist deutlich zu erkennen, dass sich die Arztpraxen entlang der Straßen sternförmig vom geographischen Zentrum der Stadt ausbreiten.

Die tatsächlich Verteilung der Arztpraxen widerspricht, wie Abbildung 4-10 gezeigt, allerdings diesen Aussagen. Auf der Karte ist eine Verteilung zu sehen, die mehreren Clustern entspricht. Es finden sich eine hohe Konzentration im Zentrum der Stadt, sowie in weite- 
ren Bereichen in Westen und Nordosten Wiens. Eine sternförmige Konzentration entlang von Straßen, die aus dem Zentrum führen ist nicht abzulesen, da beispielsweise keine Sternarme vom Zentrum in den Osten führen. Diese Aufgabe stellt ein schwieriges Beispiel dar, da es herausfordernd sein kann, die Verteilung der Arztpraxen durch ein Muster zu beschreiben. $90 \%$ der Testpersonen stimmten dabei fälschlicherweise der Aussage der Interpretationshilfe zu.

Während der Aufgabenauflösung gaben viele Testpersonen zu, sich von den Inhalten der Interpretationshilfe leiten haben zu lassen. Bei der Besprechung der Auflösung meinte beispielsweise eine Testperson, sie hätte "nicht daran gedacht, dass der Text nicht stimmen könnte" (TP 80). Auch erklärt eine Testpersonen, sie "erkenne es einfach nicht" (TP 77) nach welcher Form die ÄrztInnen in Wien verteilt sind, und deshalb die Interpretationshilfe als Unterstützung herangezogen wurde (TP 77).

Die Beschreibung der Interpretationshilfe führte dazu, dass ein gewisses Muster "verstärkend wahrgenommen" (TP 87) wurde. Auch die Verwendung des Worts "sternförmig"(TP 85) in der Interpretationshilfe als auch in der zu bewertenden Aussage verleitet dazu, die Aussage als korrekt zu bewerten. Auch die Verknüpfung mit scheinbarem Vorwissen wurde als Grund für die vorgenommene Bewertung angegeben. Als "Wiener ist man eher geneigt" (TP 83) der Aussage zuzustimmen, da man den radialen Stadtaufbau und den danach verlaufenden Straßen mit der Verteilung der Arztpraxen gleichsetzt.

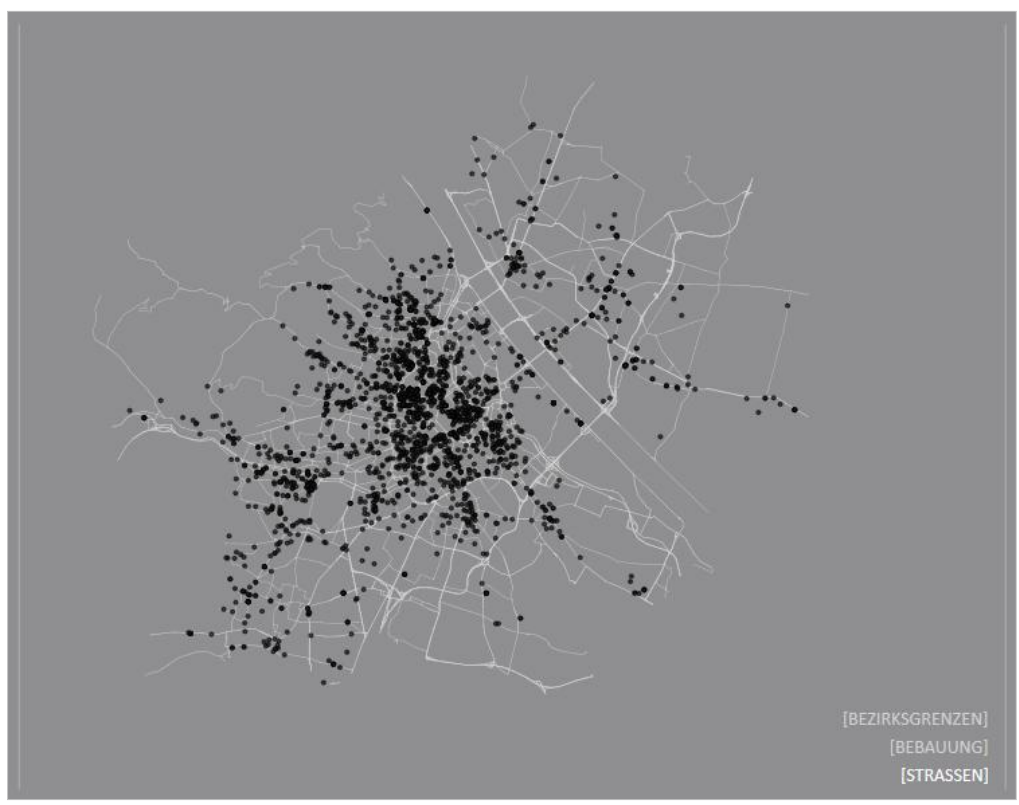

Abbildung 4-10 Verteilung der Arztpraxen Wien in Bezug auf das Straßennetz (Screenshot KIT-Karte)

Diese Begründungen lassen darauf schließen, dass die Interpretationshilfe, vor allem bei unterwarteten bzw. schwer benennbare Zusammenhänge nicht genauer hinterfragt wird. Sie wird von den Testpersonen eher als Bestätigung und Deutung gesehen, wodurch die falschen Aussagen kaum erkannt wurden. Anzumerken ist hier jedoch, dass auch ohne Beeinflussung der Interpretationshilfe in Szenario 2 nur ein Teilnehmender die Aussage richtig bewertet hat. Vermutlich ist die Beschreibung zum räumlichen Muster der Verteilung 
für viele Testpersonen nicht eindeutig.

Dass Zusammenhänge von den Testpersonen nur dann korrekt erkannt werden, wenn diese auch korrekt von der Interpretationshilfe beschrieben werden, ist nur unter Vorbehalt gültig. Betrachtet man die entsprechenden Nennungen zu korrekt und absichtlich falsch beschriebener Aussagen, ist diese Annahme korrekt. Nicht zutreffend ist sie auf jene Erklärungstexte, die keine Irreführung, sondern lediglich für die Interpretation der Zusammenhänge wenig relevante Informationen enthalten.

\section{Unterhypothese 1.2}

Interpretationshilfe beeinflusst vor allem die Informationsaufnahme der Neulinge.

Der Vergleich zwischen den abgegebenen Bewertungen der Neulinge im Vergleich zu den ExpertInnen zeigt, welche der beiden Testgruppen sich stärker an die von der Interpretationshilfe gemachten Aussagen hält.

Tabelle 4-3 stellt den Anteil der Antworten die sich an die Aussagen der Interpretationshilfe halten, zwischen Neulingen und ExpertInnen gegenüber. Um die "Interpretationshilfetreuen" Antworten herauszufiltern, werden die nicht erkannten Falschaussagen (5c, 5f) sowie die von der Interpretationshilfe unzureichend beschriebenen Zusammenhänge, die mit "weiß nicht" bewertetet wurden (5h), als richtig gewertet.

Wie bereits im Zuge der Bewertung von Unterhypothese 1.1 beschreiben wird, hat kein Teilnehmender den von der Interpretationshilfe nicht beschriebenen Zusammenhang in Aussage 5h) mit "weiß nicht" beurteilt. Weder die Neulinge noch die ExpertInnen haben sich in diesem Fall sehr stark von den Inhalten der Interpretationshilfe leiten lassen. Sehr stark ist allerdings der Einfluss der Interpretationshilfe auf Aussage 5f) zu erkennen, dem bis auf eine Testpersonen alle folgen. Hier ist zu erkennen, dass $100 \%$ der Neulinge und 90 \% ExpertInnen folgen dieser Aussage. Ein Neuling gibt dazu an: "Ich habe eigentlich fast nur die Texte gelesen. [..] hab ich die Karten eigentlich nicht verwendet weil mir der Text mehr oder weniger eh alles gesagt hat." (TP 81

\begin{tabular}{|c|c|c|}
\hline FRAGE & NEULINGE [\%] & EXPERTINNEN [\%] \\
\hline $5 a$ & 90 & 60 \\
\hline $5 b$ & 100 & 90 \\
\hline $5 c>" w a h r "$ & 40 & 40 \\
\hline $5 d$ & 100 & 60 \\
\hline $5 e$ & 100 & 100 \\
\hline $5 f>" w a h r "$ & 100 & 90 \\
\hline $5 g$ & 100 & 90 \\
\hline $5 \mathrm{~h}>$ >weiß nicht" & 0 & 0 \\
\hline $5 \mathbf{i}$ & 100 & 60 \\
\hline Gesamt & $80 \%$ & $62 \%$ \\
\hline
\end{tabular}

Tabelle 4-3

"Richtige" Antworten der Neulinge und Expertlnnen in Bezug auf die enthaltenen Informationen der Interpretationshilfe (eigene Darstellung) 
Die Antworten der Neulinge halten sich im Gegensatz zu den ExpertInnen deutlich häufiger an die Inhalte der Interpretationshilfe. Die Unterhypothese kann daher nicht verworfen werden.

Die Hypothese, dass die Verwendung einer Interpretationshilfe zur Interpretation von bestimmten Inhalten führt, kann nicht verworfen werden. Es werden tendenziell die Inhalte so wahrgenommen, wie sie von der Interpretationshilfe beschrieben werden. Das trifft vor allem bei der Verwendung von KIT-Karten durch Neulinge zu.

\subsubsection{Hypothese 2}

Durch die Verwendung einer Interpretationshilfe werden die dargestellten Inhalte häufiger korrekt interpretiert.

Der Vergleich der richtig bewerteten Aussagen zwischen Szenario 1 und Szenario 2 gibt Aufschluss darüber, ob die Interpretationshilfe zu einer korrekteren Interpretation der in der KIT-Karte enthaltenen Inhalte führt.

Insgesamt wurden in Szenario 173 \% richtige Bewertungen abgeben. In Szenario 2 sind $60 \%$ der Aussagen korrekt bewertet. Bei neun Aussagen unter Frage 5 entspricht das durchschnittlich 6,6 richtigen Antworten in Szenario 1 und 5,4 richtige Antworten pro Personen in Szenario 2.

Mit einem Plus von durchschnittlich 1,20 richtigen Antworten pro Person in Szenario 1 gegenüber Szenario 2, kann die Hypothese angenommen werden

Wie die Hypothese bei differenzierterer Betrachtung zu bewerten ist, zeigen die Unterhypothesen.

\section{Unterhypothese 2.1}

Die Interpretationshilfe verbessert vor allem die Interpretation der Neulinge.

Hinsichtlich einer differenzierteren Betrachtung der Ergebnisse aus Frage 5 zeigt Abbildung 4-11, wie viele richtige Bewertungen pro Szenario und Testgruppe gegeben wurden. Bei den ExpertInnen zeigt sich beim Einsatz der Interpretationshilfe keine Verbesserung der Ergebnisse sondern bleibt unverändert. Anders sieht es bei den Ergebnissen der Neulinge aus. Diese verbessern sich bei dem Einsatz der Interpretationshilfe deutlich. 


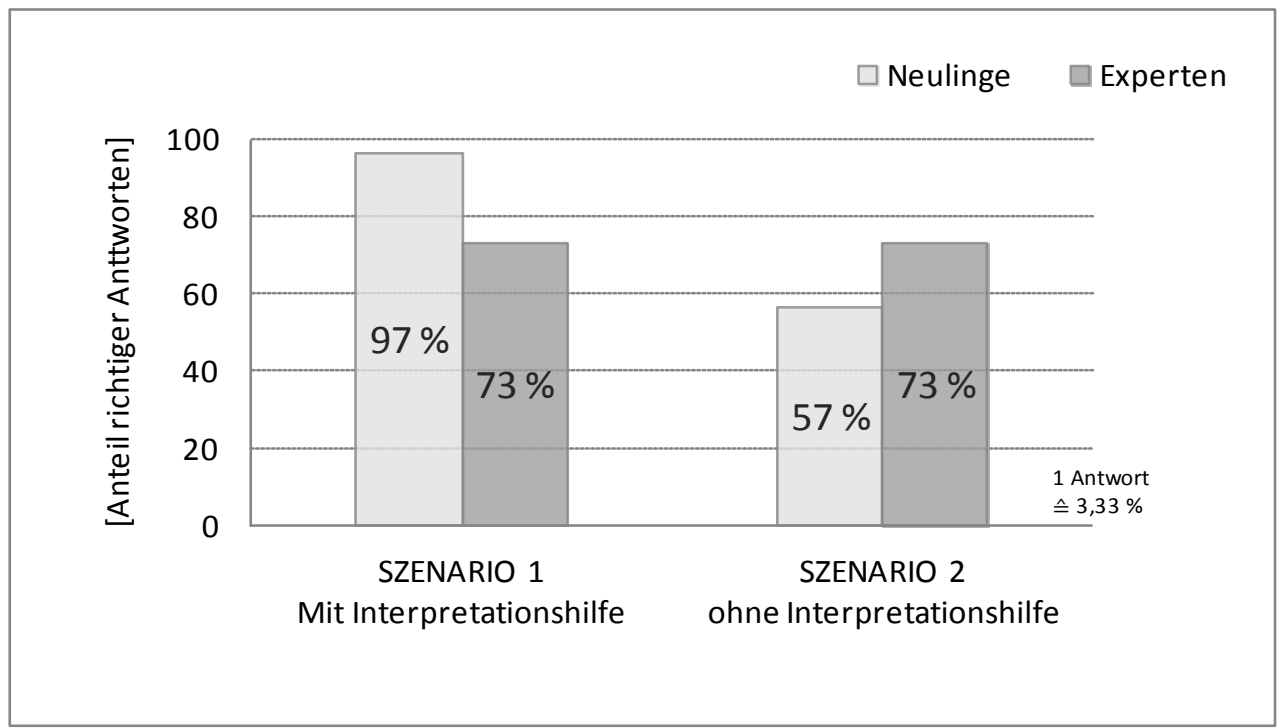

Abbildung 4-11 Richtige Antworten in Szenario 1 und Szenario 2 nach Testgruppen (eigene Darstellung)

Mit einem Plus von 40 \% (durchschnittlich 1,20 richtigen Antworten pro Neuling) ist im Vergleich zu unveränderten Ergebnissen der ExpertInnen in Szenario 1 die Unterhypothese nicht zu verwerfen.

\section{Unterhypothese $\mathbf{2 . 2}$}

Der positive Einfluss der Interpretationshilfe tritt vor allem bei unbekannten Orten auf.

Die Verbesserung der Interpretation wird weiter hinsichtlich ihres Einflusses auf Karten über bekannte und unbekannte Orte untersucht. Abbildung 4-12 zeigt die richtigen Antworten, die pro Person hinsichtlich bekannter und unbekannten Orten je Szenario abgeben wurden. Wie zu erkennen ist, werden in beiden Szenarien mehr richtige Bewertungen zu Wien als zu Kopenhagen abgegeben. Zu beachten ist allerdings, dass aufgrund der Konzeption des Fragebogens um eine Frage mehr zu Wien als zu Kopenhagen existiert. Zieht man, um diesen Umstand auszugleichen, 1 richtige Antwort pro Person für Wien ab, schneidet Kopenhagen trotzdem in der Gesamtwertung immer noch schlechter ab.

Betrachtet man jedoch die Veränderungen zwischen Szenario 1 und Szenario 2 für jeweils Wien und Kopenhagen, kann Aufschluss über den Einfluss der Interpretationshilfe erlangen. Bezogen auf Wien werden bei Einsatz der Interpretationshilfe um 0,5 mehr richtige Antworten pro Person gegeben. Für Kopenhagen steigen die richtigen Bewertungen im Durchschnitt um 0,7 korrekte Angaben pro Person.

Die Verwendung der Interpretationshilfe führt dazu, dass sich die richtigen Antworten zu Kopenhagen in Szenario 1 erhöhen. 


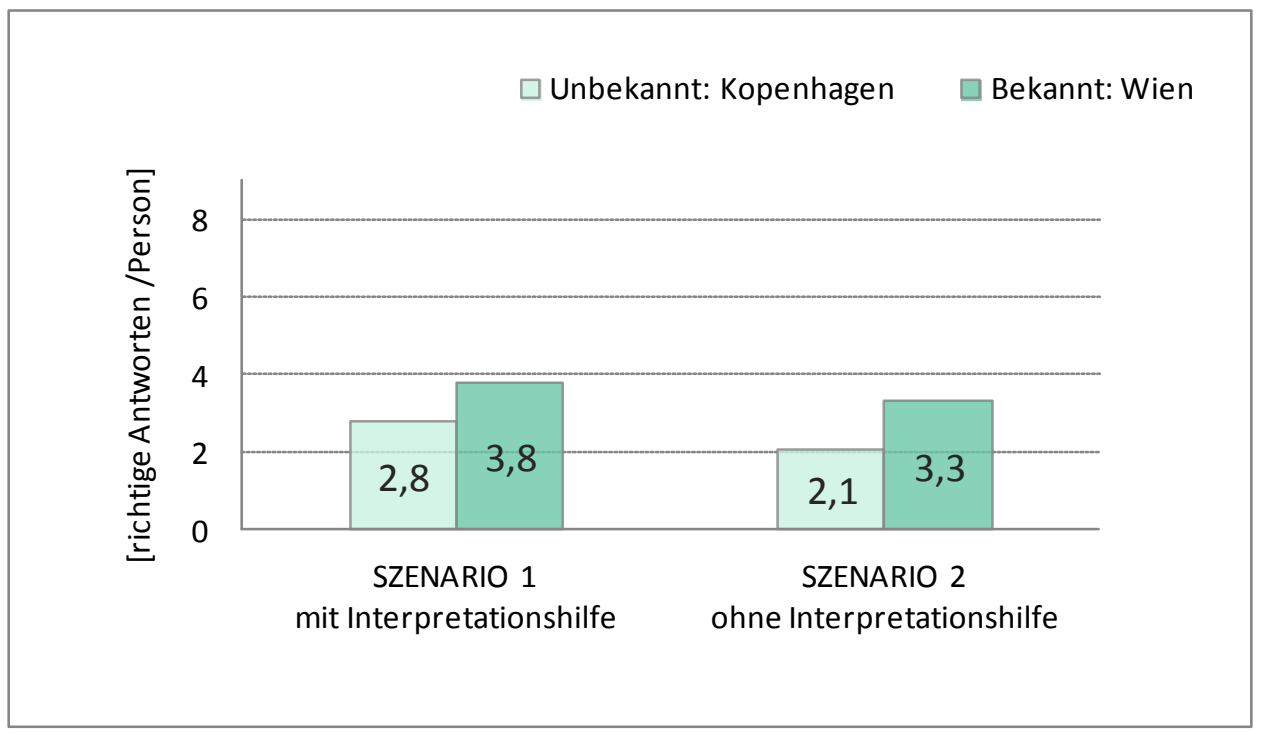

Abbildung 4-12 Richtige Antworten zu Kopenhagen

(eigne Darstellung)

Allgemein kann die Hypothese, dass bei Verwendung von Interpretationshilfen die dargestellten Inhalte häufiger korrekt interpretiert nicht verworfen werden. Im Vergleich zwischen den beiden Testgruppen wird vor allem die Interpretation der Neulinge verbessert. In Bezug auf die Bekanntheit von Orten unterstützt die Interpretationshilfe bei unbekannten Orten.

\subsubsection{Hypothese 3}

Die Verwendung einer Interpretationshilfe führt zu einem verminderten Explorieren in der Karte.

Zur Auswertung von Hypothese 3 wird das Klickverhalten und die verbrachte Zeit bei der Verwendung der KIT-Karte in Szenario 1 und Szenario 2 beobachtet. Die drei Unterhypothesen werden zur Beurteilung dieser Hypothese herangezogen.

\section{Unterhypothese 3.1}

Es werden wenig neue Bereiche neben den von der Interpretationshilfe angesprochenen Bereichen aufgerufen.

Im Zuge des Usability-Tests werden bei der Führung durch die Karte bewusst wichtige Kartenkombinationen von der Interpretationshilfe ausgelassen. Dieses hat den Zweck, um zu überprüfen, ob die Testpersonen selbstständig nach Informationen in der Karte suchen.

Ausgelassen wird die Aussageschicht zu den Eigentumspreisen in Kopenhagen sowie die Ortsdiagrammkarte zur Verteilung der speziellen Facharztgruppen in jeweils Wien und Kopenhagen. Alle weiteren Aussageschichten der KIT-Karten werden durch verschiedene 
Kombinationen zumindest einmal angesprochen und somit den Testpersonen präsentiert. Laut Aufgabenstellung in Anhang D sind die Testpersonen direkt dazu aufgefordert, Informationen zur Verteilung der Arztpraxen in Bezug auf die Eigentumspreise in Kopenhagen zu finden. Demnach sollte dieser Inhalt von allen Testpersonen gesucht werden. Die Betrachtung der einzelnen Facharztgruppen wird durch die Aufgabenstellung nicht gefordert, sondern wird als Zusatzinformation in der Karte bereitgestellt. Tabelle 4-4 zeigt den Prozentsatz der Personen, die die von der Interpretationshilfe nicht angesprochenen Bereiche aufgerufen haben.

\begin{tabular}{|c|c|c|c|}
\hline KARTENINHALTE & SZENARIO 1 & SZENARIO 2 & \multirow{5}{*}{$\begin{array}{l}\text { Tabelle 4-4 Anteil der Personen } \\
\text { nach Szenario, die von der Interpre- } \\
\text { tationshilfe nicht präsentierte Inhal- } \\
\text { te aufgerufen haben } \\
\text { (eigene Darstellung) }\end{array}$} \\
\hline Eigentumspreise Kopenhagen & $70 \%$ & $80 \%$ & \\
\hline FachärztInnen Kopenhagen & $50 \%$ & $60 \%$ & \\
\hline FachärztInnen Wien & $50 \%$ & $60 \%$ & \\
\hline & & 1 Person $\cong 10 \%$ & \\
\hline
\end{tabular}

In Szenario 1 haben deutlich weniger Testpersonen diese Bereiche aufgerufen als sie beim selbstständigen Erkunden der Testpersonen in Szenario 2 entdeckt wurden. Dies trifft ohne Ausnahme auf alle der drei nicht präsentierten Aussageebenen zu. Vor allem der Verteilung der Facharztpraxen in beiden Städten wird wenig Beachtung geschenkt. Das kann teilweise daraus resultieren, dass sich die Testpersonen stark auf die Aufgabenstellung konzentriert haben und deswegen an Informationen, die darüber hinaus gehen, nicht interessiert waren. Einige Testperson (TP 81, 69) bestätigen diesen Zusammenhang und geben an, diese Information als "irrelevant" (TP 69) für die Bearbeitung der Aufgabe eingestuft zu haben. Dass allerdings die Aussageebene über die Eigentumspreise in Kopenhagen von mehr als einem Viertel der Testpersonen nicht aufgerufen wurde, kann nicht durch diesen Umstand erklärt werden.

In Szenario 1 werden wenig neue Bereiche selbstständig aufgerufen, wodurch die Unterhypothese anzunehmen ist.

\section{Unterhypothese 3.2}

Ist eine Interpretationshilfe vorhanden, verbringen die Testpersonen weniger Zeit mit der Verwendung der Anwendung.

Die Zeitmessung hält fest, wie lange sich die Testpersonen mit der Karte beschäftigen, um alle nötigen Informationen zu erfassen. Erfasst wird die Zeit, die zwischen drücken des "Start"-Buttons für den Start der KIT-Karte bis zum Beenden der Anwendung über den "Anwendung-Beenden"-Buttons vergeht. Abbildung 4-13 stellt die gemessenen Werte gegenüber. Die Neulinge verbringen durchschnittlich weniger Zeit mit der Anwendung. Auch ist in dieser Gruppe der niedrigste Minimalwert zu finden. 


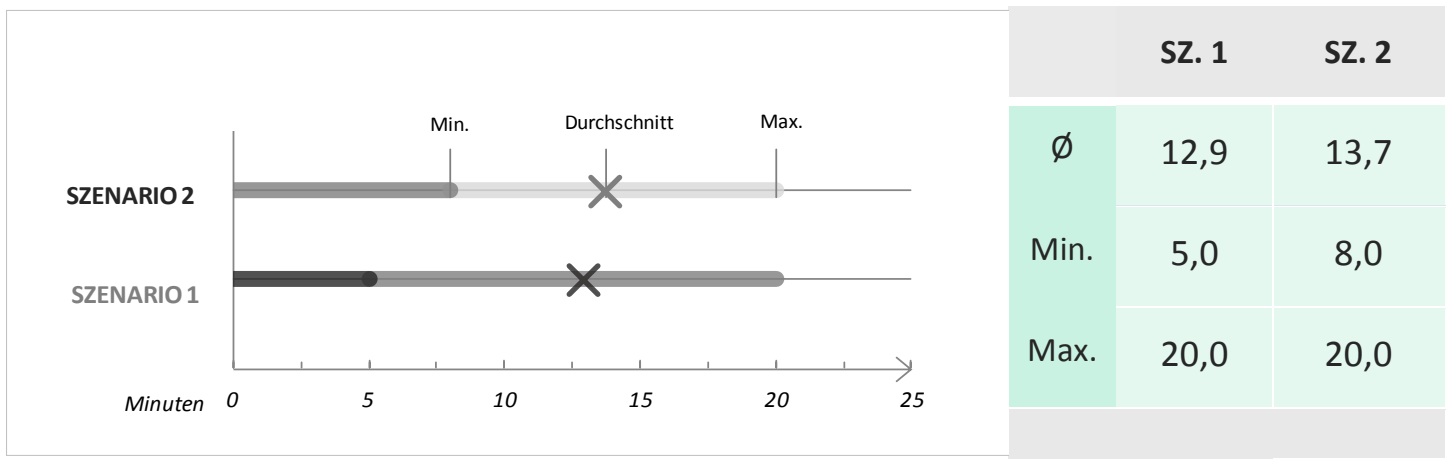

Abbildung 4-13 Aufgebrachte Zeit zur Verwendung der Karte in Szenario 1 und Szenario 2 (eigne Darstellung)

Mit einer verkürzten durchschnittlichen Dauer von 0,8 Minuten pro Person in Szenario 1 kann die Unterhypothese angenommen werden.

\section{Unterhypothese 3.3}

Die Interpretationshilfe vermindert vor allem das Explorieren der Neulinge.

Das Explorieren in der KIT-Karte setzt sich aus dem Ansprechen von neuen Bereichen und aus dem Zeitaufwand zusammen.

\section{a) Ansprechen neuer Bereiche}

Tabelle 4-5 zeigt den Anteil der Personen je Testgruppe, die die von der Interpretationshilfe ausgelassenen Bereiche angesprochen haben und die Veränderung zwischen den beiden Szenarien. In der Testgruppe der Neulinge vermindert sich im Vergleich zwischen Szenario 1 und 2 das Explorieren in zwei von drei Fällen. In der Testgruppe der ExpertInnen wird dieses nur in einem Bereich vermindert.

\begin{tabular}{|c|c|c|c|c|c|c|}
\hline & \multicolumn{2}{|c|}{ SZENARIO 1} & \multicolumn{2}{|c|}{ SZENARIO 2} & \multicolumn{2}{|c|}{ VERÄNDERUNG } \\
\hline & Neulinge & ExpertInnen & Neulinge & ExpertInnen & Neulinge & ExpertInnen \\
\hline $\begin{array}{l}\text { Eigentumspreise } \\
\text { Kopenhagen }\end{array}$ & $60 \%$ & $80 \%$ & $60 \%$ & $100 \%$ & - & $-20 \%$ \\
\hline $\begin{array}{l}\text { FachärztInnen } \\
\text { Kopenhagen }\end{array}$ & $40 \%$ & $60 \%$ & $60 \%$ & $60 \%$ & $-20 \%$ & - \\
\hline $\begin{array}{l}\text { FachärztInnen } \\
\text { Wien }\end{array}$ & $60 \%$ & $40 \%$ & $80 \%$ & $40 \%$ & $-20 \%$ & - \\
\hline
\end{tabular}

Tabelle 4-5 Anteil der Personen, die die von der Interpretationshilfe nicht angesprochenen Bereiche aufrufen (eigene Darstellung)

Das Ansprechen neuer Bereiche wird demnach bei den Neulingen durch den Einsatz der Interpretationshilfe mehr eingeschränkt als bei den ExpertInnen. 


\section{b) Zeitaufwand}

Abbildung 4-14 zeigt, wie lange sich die Testgruppen durchschnittlich mit der Karte beschäftigen. Unabhängig vom Szenario beschäftigen sich ExpertInnen im Durchschnitt um fast 1 Minute länger mit der Karte als Neulinge. Betrachtet man die Veränderung des durchschnittlichen Zeitaufwands der Neulinge zwischen Szenario 1 und Szenario 2, verkürzt sich dieser nicht, sondern verlängert sich.

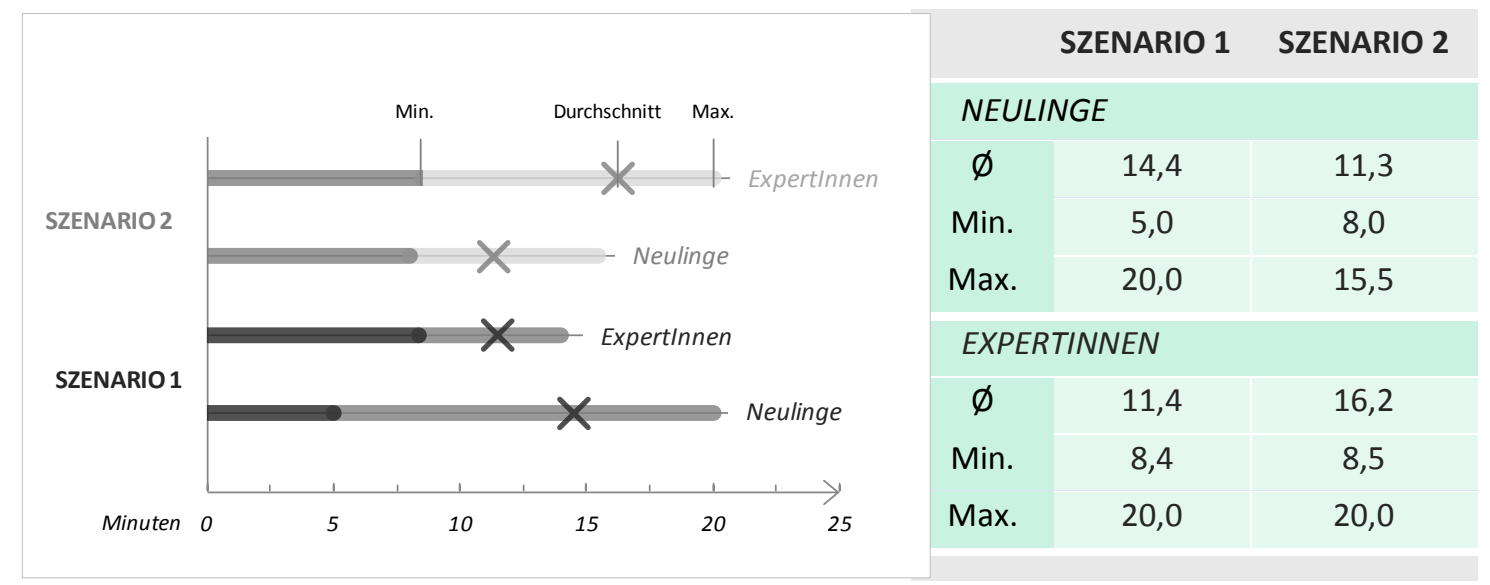

Abbildung 4-14 Aufgebrachte Zeit zur Verwendung der Karte nach Szenario (eigene Darstellung)

Mit einem Plus von 3,2 Minuten trifft es demnach nicht zu, dass die Neulinge durch Verwendung der Interpretationshilfe weniger Zeit mit der Anwendung verbringen. Im Fall der ExpertInnen ist dies Annahme jedoch zutreffend. In dieser Testgruppe verkürzt sich die durchschnittlich aufgebrachte Zeit um 4,8 Minuten.

Zusammenfassend werden beim Einsatz der Interpretationshilfe von den Neulingen weniger Bereiche selbstständig aufgerufen als von den ExpertInnen. Die Zeit, mit der sich mit der Anwendung beschäftigt wird, verkürzt sich durch die Interpretationshilfe bei den Neulingen jedoch nicht. Die Unterhypothese kann also nur im Bezug auf die Explorationen neuer Bereiche angenommen werden. Im Bezug auf eine verkürzte Verweildauer in der Karte wird die Unterhypothese verworfen.

In Bezug auf eine undifferenzierte Betrachtung der Testpersonen kann die Hypothese nicht verworfen werden. Durch die Interpretationshilfe wird das Explorieren sowohl durch die Anzahl der selbstständig aufgerufenen Bereiche als auch durch die insgesamt verwendete Zeit minimiert. Es ist allerdings nicht vollständig zutreffend, dass besonders das Explorieren der Neulinge durch den Einsatz der Interpretationshilfe eingeschränkt wird. Von dieser Testgruppe werden nur weniger Bereiche selbstständig aufgerufen, die aufgewendete Zeit wird jedoch nicht verkürzt. 


\subsubsection{Hypothese 4}

Die Testpersonen fühlen sich von der Interpretationshilfe bevormundet.

Auf die spontane Frage, ob die Interpretationshilfe bei der Bewertung der Aussagen in Frage 5 hilfreich war, äußerten sich fast alle Testteilnehmenden positiv über die Interpretationshilfe. Nur eine Expertin gibt an, die Interpretationshilfe sei für sie persönlich nicht unbedingt hilfreich gewesen. Sie ist die Interpretationshilfe nur "schnell durchgegangen" (TP 70) und habe die Zusammenhänge durch ihre eigene Erkundung festgestellt. Sie bergründet ihre Vorgehensweise damit, dass sie ohne die Interpretationshilfe die Aufgaben "genauso gut lösen" (TP 70) hätte können. Allerdings wird trotzdem positiv eingeräumt, dass die Interpretationshilfe allgemein "vielleicht eine Hilfe [ist] wenn man weniger Zeit hat oder wenn man nicht so genau ist". Man "erklickt nicht alles" (TP 70) was man aus der Karte herauslesen könnte.

Tabelle 4-6 zeigt eine detaillierte Beschreibung der Meinung über die Interpretationshilfe. Die Testpersonen bewerteten diese hinsichtlich der Multiple-Choice-Auswahl und konnten dabei Mehrfachnennungen und somit positive als auch negative Meinungen abgeben. Bezogen auf alle Aussagen über die Interpretationshilfe gibt es lediglich $40 \%$ bzw. $20 \%$ negative Urteile. Diese stehen im deutlichen Gegensatz zu 100 \% bzw. $10 \%$ positiver Bewertungen.

\begin{tabular}{|c|c|c|c|c|}
\hline & $\begin{array}{c}\text { "Hilft gezielter } \\
\text { wahrzunehmen" (1) }\end{array}$ & $\begin{array}{c}\text { "Nur Offensichtliches } \\
\text { beschrieben" (2) }\end{array}$ & $\begin{array}{l}\text { "Nicht umfassend } \\
\text { genug" (3) }\end{array}$ & $\begin{array}{l}\text { "Sonst Inhalte } \\
\text { unverständlich" (4) }\end{array}$ \\
\hline Neulinge & $100 \%$ & $0 \%$ & $0 \%$ & $0 \%$ \\
\hline ExpertInnen & $100 \%$ & $80 \%$ & $40 \%$ & $20 \%$ \\
\hline \multirow[t]{2}{*}{ Gesamt } & $100 \%$ & $40 \%$ & $20 \%$ & $10 \%$ \\
\hline & & & & 1 Person $\triangleq 10 \%$ \\
\hline \multicolumn{5}{|c|}{ Usability Test - Frage 9} \\
\hline \multicolumn{5}{|c|}{ (1) Die textlichen Kommentare helfen die wesentliche Aussage der Karte gezielter wahrzunehmen. } \\
\hline \multicolumn{5}{|c|}{ (2) In den textlichen Kommentaren wird ohnehin nur das beschrieben, was auf der Karte leicht zu erkennen ist. } \\
\hline \multicolumn{5}{|c|}{ (3) Die textlichen Kommentare sind nicht umfassend genug, da noch viel mehr aus den Karten herauszulesen ist. } \\
\hline
\end{tabular}

Tabelle 4-6 Bewertung der Interpretationshilfe durch Multiple-Choice-Auswahl (eigene Darstellung)

Bei einer Betrachtung von allen Testpersonen ist aufgrund des hohen Anteils positiver Bewertungen die Hypothese nicht zutreffend.

\section{Unterhypothese 4.1}

Die Interpretationshilfe wird hauptsächlich von ExpertInnen negativ beurteilt.

Wie weiter aus Tabelle 4-6 zu entnehmen ist, werden die negativen Bewertungen ausschließlich von ExpertInnen abgegeben. Neben $100 \%$ bzw. $20 \%$ positiven Stellungnahmen beurteilen 80 \% bzw. $20 \%$ der ExpertInnen die Interpretationshilfe dennoch auch kritisch. Anzumerken ist, dass keine der ExpertInnen eine rein negative Bewertung abgege- 
ben hat, sonders diese in Begleitung von Positiven erfolgte.

"Durch den Text [hat man] schnell erkannt, was man auf der Karte sieht. Einerseits wurde eben genau das beschrieben, aber andererseits nur das Große und Ganze weil Details kann man in so einem kurzen Text nicht beschreiben." (TP 75)

"Das wesentliche ist [durch die Interpretationshilfe] herausgearbeitet. Man könnte immer irgendwie noch mehr herauslesen."(TP 70)

"Eigentlich beschreiben sie das was ich sehen kann, aber ich will nicht sagen dass sie unnötig ist. [..] Man kann immer noch mehr aus Karten heraus interpretieren als was in Texten steht - eigentlich ist nie eine Beschreibung eines Texts umfassend genug." (TP 89)

Von den Neulingen werden ausschließlich positive Bewertungen abgegeben, zu den positiven Bewertungen der ExpertInnen mischen sich auch kritische Bewertungen. Die Unterhypothese ist demnach anzunehmen.

Bezüglich einer allgemeinen Bewertung der Interpretationshilfe ist die Hypothese zu verwerfen, da alle Testpersonen die Interpretationshilfe entweder vollständig oder teilweise positiv beurteilen. Zutreffend ist jedoch, dass die negativen Kommentare ausschließlich von den ExpertInnen gekommen sind.

\subsubsection{Hypothese 5}

Die Interpretationshilfe wird von den Testpersonen als hilfreich und unterstützend wahrgenommen.

Für die Beantwortung der Hypothese ist sowohl die Bewertung zur Nützlichkeit der Interpretationshilfe, die subjektive Einschätzung des Schwierigkeitsgrads der Aufgabenlösung als auch die neuerliche Bewertung der Benützung der KIT-Karten ausschlaggebend.

\section{a) Bewertung zur Nützlichkeit der Interpretationshilfe}

Wie bereits in Kapitel 4.4.4.4 beschrieben, bejahen fast alle Teilnehmenden die hilfreiche Wirkung der Interpretationshilfe:

"Hilfreich [..] damit man weiß worum es geht, sonst schaut man nur so die Karte an." (TP 66)

"Ergänzend [..] wenn man schnell drauf schaut ist es auf jeden Fall hilfreich". (TP 88)

"Viel angenehmer weil man weiß worauf man schauen muss und was man suchen könnte." (TP 77)

Die Interpretationshilfe suggeriert: "Hey schau dir das hier an und schau dir das dort an [..] Ich habe das Gefühl ich habe die Inhalte so schneller erfasst." (TP 89)

Wie bereits aus Tabelle 4-6 zu entnehmen ist, wird die Interpretationshilfe von allen Testpersonen zumindest teilweise positiv beurteilt. $100 \%$ der Teilnehmenden sehen die Interpretationshilfe als 
"hilfreich". Unbedingt notwendig scheint sie dennoch nicht zu sein, denn nur eine Testperson gibt an, dass sie ohne die Interpretationshilfe die Inhalte nicht verstanden hätte (TP 66). Die Antwort dieses Experten kann jedoch so verstanden werden, dass sie sich darauf bezieht, dass genau die bestimmt gefragten Zusammenhänge herausgelesen werden und die Karte nicht hinsichtlich anderer Gesichtspunkte untersucht wird. Die positive Einstellung der Testpersonen zur Nützlichkeit der Interpretationshilfe ist eindeutig zu erkennen.

\section{b) subjektive Einschätzung des Schwierigkeitsgrads der Aufgaben}

Das subjektive Schwierigkeitsempfinden zu Lösen von Aufgabe 5 gibt Hinweise zur unterstützenden Wirkung der Interpretationshilfe. Abbildung 4-15 zeigt je Szenario wie schwer es den Testteilnehmenden gefallen ist, die Aussagen zu bewerten.

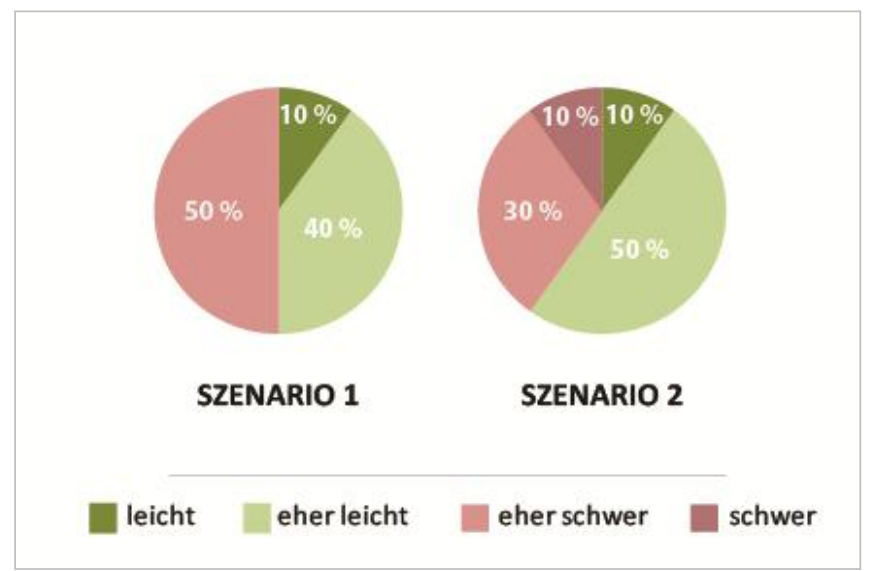

Abbildung 4-15 Einschätzung des Schwierigkeitsgrad Lösen der Aufgaben (eigene Darstellung)

Die rot schattierten Segmente weisen darauf hin, dass das Lösen der Aufgaben als schwieriger empfunden wurde. Die grünen Segmente zeigen, dass die Teilnehmenden keine oder kaum Schwierigkeiten bei der Lösung der Aufgabe empfanden. Hinsichtlich der subjektiven Einschätzung zur Aufgabenlösung ist durch den Einsatz der Interpretationshilfe keine Vereinfachung zu erkennen. Dennoch erklären Teilnehmende aus Szenario 1, dass sie durch die Texte der Interpretationshilfe die Bewertung als leichter empfunden haben. Begründung dazu sind beispielsweise:

"Dadurch, dass das Wissen schon aufbereitet war, hab ich's mir einfach besser gemerkt, als wenn ich selber die Schlüsse ziehen muss" (TP 87).

"Was in der Interpretationshilfe steht ist eigentlich genau das, was [in Frage 5] steht" (TP 85).

Teilnehmende aus Szenario 2 begründen die Schwierigkeiten bei der Aufgabenlösung beispielsweise damit, dass "nicht ganz klar [war] worauf die Aufgabenstellung hinauswill" (TP 69). Es war deswegen nicht klar, was das Ziel der Untersuchung der Karte sein soll. Auch seien "die Zusammenhänge nicht immer eindeutig" (TP 67) gewesen. Manche Bereiche (bspw. Verteilung der Arztpraxen an der Bebauung (TP 78) nicht angesprochen wurden und daher die dazugehörige Aussage schwierig zu bewerten war.

Die Testpersonen in Szenario 2 wurden gefragt, ob sie sich eine Unterstützung zum Interpretieren der Inhalte wünschten. Keiner der Testpersonen nannte eine textliche Unterstützung im Sinne der Interpretationshilfe. Viel mehr wurden Verbesserungsvorschläge zum Layout der Karte und ihren 
Funktionen gemacht um die Verwendung der Karte zu verbessern (siehe Kapitel 4.4.6)

\section{c) neuerliche Bewertung der Benützung der KIT-Karten}

In Kapitel 4.4.4.5 b) wurde die allgemeine Einschätzung der Testpersonen zur Verwendung von KIT Karten bereites beschrieben. Abbildung 4-16 zeigt, wie die neuerliche Bewertung aussieht, wenn eine Berücksichtigung der Interpretationshilfe erfolgt. Die Testteilnehmenden wurden dafür gebeten, die Verwendung von KIT-Karten unter der Annahme zu bewerten, dass in allen KIT-Karten ab sofort Interpretationshilfen vorhanden wären.

Bis auf den Minimalwert, der in Bezug auf das "Erfassen von konkreten Informationen" besteht, verschieben sich alle weiteren Minimum-, Maximum -und Durchschnittswerte nach rechts. Folglich wird bei Einsatz einer Interpretationshilfe der informative, einfache, kommunikationsorientierte Charakter hervorgehoben. Der positive Einfluss der Interpretationshilfe ist nach dieser Betrachtung deutlich abzulesen. Eine Testperson beschreibt ihre veränderte Bewertung damit:

"Der Text macht es auf jeden Fall leichter. Weil du auf das Geschriebene achtest und nicht selbst darauf angewiesen bist, etwas aus der Karte herauszulesen." (TP 77)

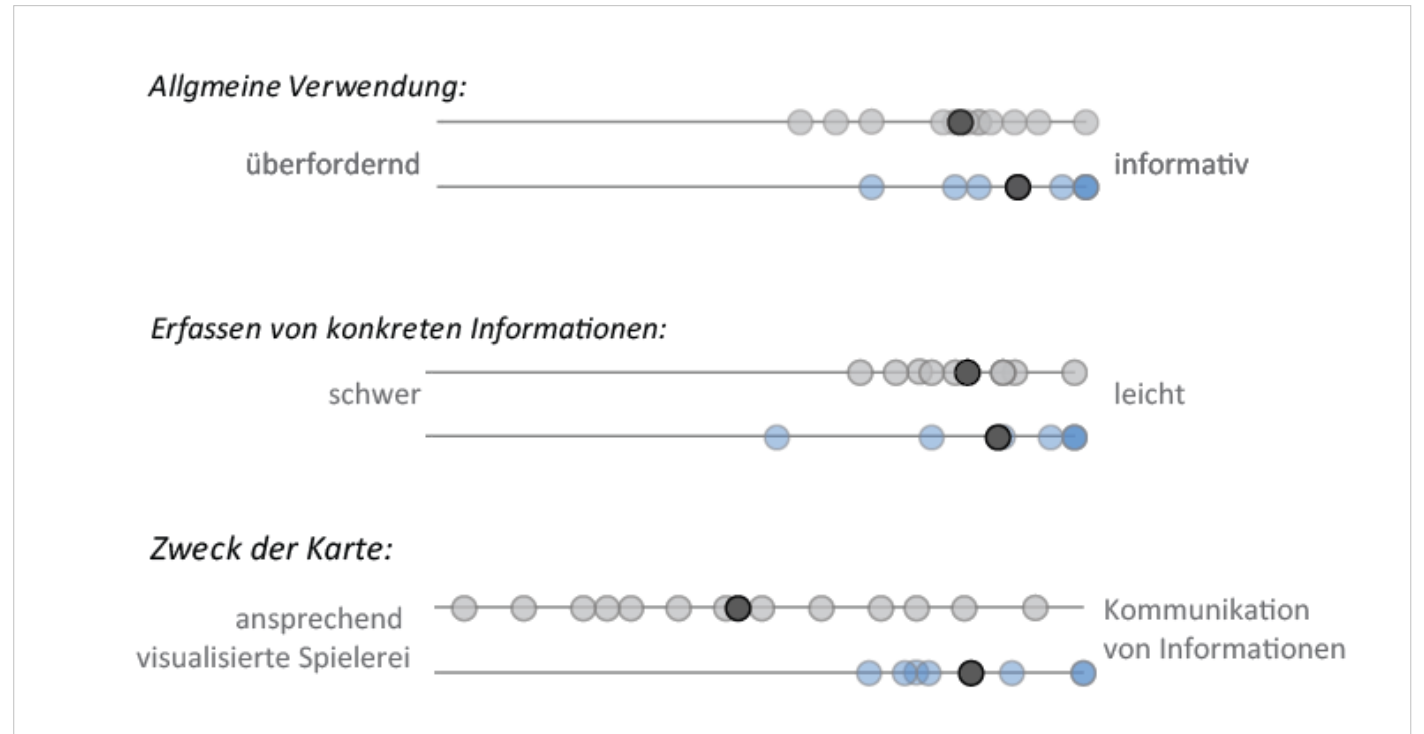

Abbildung 4-16 Einschätzung über die Verwendung von KIT-Karten unter Berücksichtigung der Interpretationshilfe (eigene Darstellung)

Zusammenfassend wird von allen Teilnehmenden die Interpretationshilfe zumindest teilweise als hilfreich bezeichnet. Dieser Einfluss ist jedoch nicht aus der subjektiven Einschätzung zur Aufgabenlösung bei Verwendung der Interpretationshilfe zu erkennen. Bei einer neuerlichen Bewertung der Verwendung von KIT-Karten verschiebt sich dennoch aufgrund der Interpretationshilfe die Wahrnehmung dieser in Richtung der Kommunikation von Informationen.

Da in den direkten Bewertungen und Einschätzungen von Interpretationshilfe in KIT-Karten der positive Tenor überwiegt, wird die Hypothese angenommen.

Inwieweit die Hypothese bei differenzierter Betrachtung hinsichtlich der verschiedenen Testgruppen und ihrer Nützlichkeit bei bekannten und unbekannten Orten zutrifft, wird durch die folgenden Un- 
terhypothesen getestet.

\section{Unterhypothese 5.1}

Die Interpretationshilfe wird hauptsächlich von Neulingen positiv beurteilt.

Wie bereits aus Tabelle 4-5 zu entnehmen ist, beurteilen vor allem Neulinge die Interpretationshilfe positiv. Niemand der Teilnehmenden dieser Testgruppe gab eine einzige negative Bewertung ab.

Da von den Neulingen im Gegensatz zu den ExpertInnen nur positive Meinungen abgegeben wurden, wird die Unterhypothese angenommen.

\section{Unterhypothese 5.2}

Die Interpretationshilfe wird vor allem bei unbekannten Orten als Hilfe empfunden.

Für die Evaluierung der Unterhypothese wird die Einschätzung über den Schwierigkeitsgrad der Aufgabenlösung differenziert und durch die direkte Bewertung der Interpretationshilfe durch die Testpersonen ergänzt.

\section{a) Beurteilung der Aufgabenlösung}

Abbildung 4-17 zeigt das subjektive Empfinden zum Schwierigkeitsgrad zu Frage 5. In Szenario 2 wird das Bewerten von Aussagen zu Kopenhagen als am schwierigsten wahrgenommen.

Anzumerken ist, dass in keinem der beiden Szenarien eine Testperson angegeben hat, die Bewertung der Aufgaben zu Wien im Vergleich zu Kopenhagen als schwieriger empfunden zu haben.

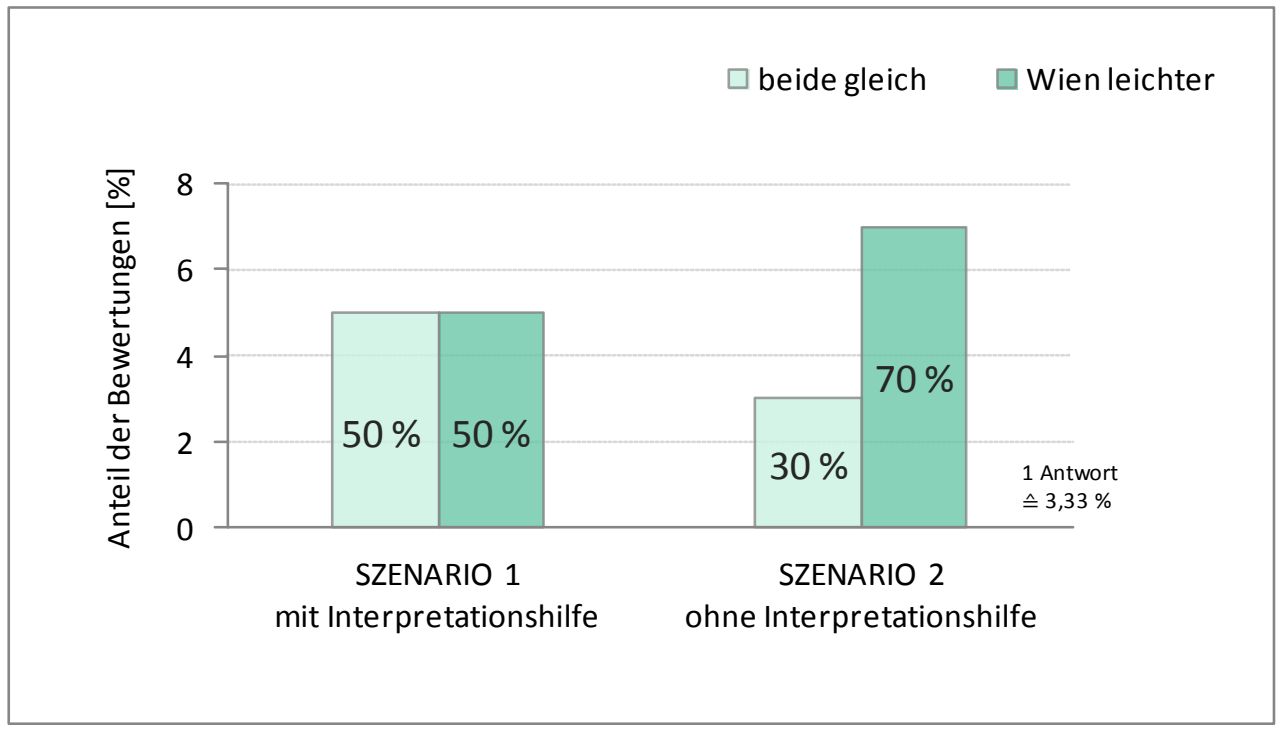

Abbildung 4-17 Subjektives Empfinden bei der Aufgabenlösung 
In Szenario 1 wurde Wien aus verschiedenen Gründen im Gegensatz zu Kopenhagen als leichter empfunden. Hauptsächlich wurde von den Testpersonen genannt, dass es ihnen erleichtert hat, dass sie sich in Wien besser auskennen $(77,85)$. Es wird sogar angegeben, dass die Fragen zu Wien " eher aus dem Kopf beantwortet" (TP 77) wurden, während bei den Fragen zu Kopenhagen sich "auf die Karte verlassen" (TP 77) wurde. Auch werden für Wien "mache Sachen logischer (85)" gesehen, weil man sich Situation "besser vorstellen kann" und "besser mit Vorwissen verbinden" (TP 88) kann. Im Gegensatz dazu wird Kopenhagen aufgrund der fehlenden geographischen Kenntnisse als schwieriger empfunden. Da man beispielsweise "nicht weiß wo das Zentrum ist" (TP 66). Als ein weiterer Grund wird genannt, dass in Kopenhagen weniger Praxen auf der Karte abgebildet sind und es somit schwieriger sei, Zusammenhänge abzulesen (TP 87,77).

In Szenario 2 gelten ähnliche Gründe für die Unterscheidung der Bewertungen zu Wien und Kopenhagen. Das Lösen der Aufgaben betreffend Kopenhagen gilt als schwerer "weil ich die Stadt nicht kenne" (TP 76). Man "wusste nicht welcher von den Bezirken das Zentrum ist" (TP 77). Im Gegensatz dazu waren die Aussagen zu Wien leichter zu beantworten "weil ich schon Kenntnisse darüber habe" (TP 84, 69). In Wien werde die Aussage der Karten "mit Bezirken verglichen, die man kennt um etwas abzuleiten" (TP 77). Die Verknüpfung mit Vorwissen wird auch dadurch bestätigt, dass überlegt wurde "wo in Wien die Ärzte sind. Ob die an großen Straßen sind oder an kleinen" (TP 78). Auch bestünde ein größeres Interesse an Wien wodurch es "genauer angeschaut [werde] weil es meine Heimatstadt ist. Da hat man einen besseren Bezug dazu" (TP 82). Durch das "Hintergrundwissen" (TP 77) zu Wien "tut man sich einfach leichter" (TP 77).

Aus der subjektiven Einschätzung der Testpersonen kann abgeleitet werden, dass eine Unterstützung bei KIT-Karten zu unbekannten Orten besonders relevant ist. Wie Tabelle 4-17 zeigt, kann durch den Einsatz der Interpretationshilfe Schwierigkeiten zu Kopenhagen zumindest teilweise reduziert werden. Der positive Einfluss der Interpretationshilfe für die Interpretation von unbekannten Orten kann daraus abgeleitet werden.

\section{b) Bewertung der Interpretationshilfe}

Wie bereits in Kapitel 4.4.4.5 dargestellt, wird die Interpretationshilfe generell von den Testpersonen als hilfreiche Unterstützung gesehen. Laut Tabelle 4-7 empfinden 44 \% der Testpersonen die Hilfe durch die Interpretationshilfe für beide Städte gleichermaßen hilfreich. Der Text helfe generell immer, "weil du weißt worauf du schauen musst - vor allem wenn man so viele Sachen verstellen kann" (TP 77). 33 \% geben an geben an, dass ihnen die Interpretationshilfe bei der Interpretation der KIT-Karten von Kopenhagen mehr geholfen hat als bei Wien. "In Wien habe ich nicht so besonders drauf geachtet, aber in Kopenhagen war ich mehr drauf angewiesen." (TP 77) Ein Grund dafür war wieder, dass Kopenhagen eine "unbekannte Stadt ist." (TP 87) Die verbleibenden $22 \%$ identifizieren die hilfreiche Wirkung eher in Bezug auf die Karten für Wien. Für Wien seien diese deshalb hilfreicher, da die Testpersonen mit denen im Text verwendeten Bezirksbezeichnungen vertraut 
sind und wissen "wo die sind" (TP 75). So konnten die Beschreibungen der Interpretationshilfe schneller in der Karte "gefunden"(TP 66) werden.

$$
\begin{gathered}
\text { für beide Städten } \\
\text { gleich hilfreich }
\end{gathered}
$$

Anteil der

Testpersonen mehr für Wien hilfreich

$22 \%$ mehr für

Kopenhagen hilfreich

$33 \%$

Tabelle 4-7 Bewertung der Interpretationshilfe hinsichtlich unterschiedlicher Städte

(eigene Darstellung)

Bezogen auf die konkrete Bewertung der Interpretationshilfe zeigt Abbildung 4-18, wie die abgegebenen Bewertungen im Zusammenhang mit dem geographischen Ort stehen. Auch hier zeigt sich die ausgeglichene positive Bewertung zu beiden Städten.

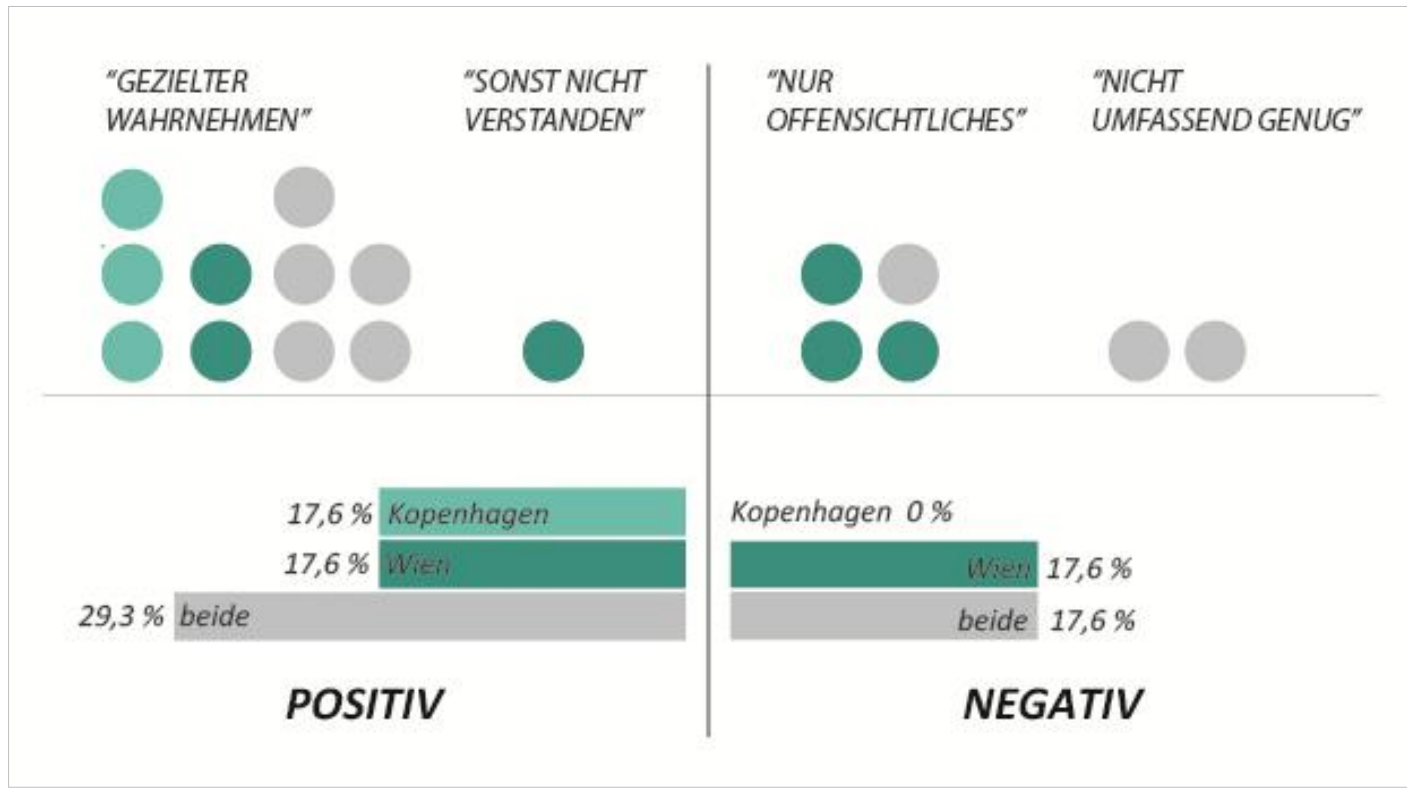

Abbildung 4-18 Bewertungen der Interpretationshilfe hinsichtlich bekannter und unbekannter Orte (eigene Darstellung)

Ist die Interpretationshilfe nicht vorhanden, wird die Bewertung zu Aussagen über Kopenhagen im Vergleich zu Wien öfter als schwieriger empfunden. Dennoch empfinden die Testpersonen die Hilfe als eher ausgeglichen zwischen beiden Städten. Da jedoch keine einzige negative Bewertung der Interpretationshilfe in Bezug auf Kopenhagen abgeben wird, wird die Unterhypothese angenommen.

Aufgrund der überwiegenden positiven Bewertungen aller Testpersonen ist die Hypothese über die hilfreiche und unterstützende Wahrnehmung der Interpretationshilfe nicht zu verwerfen. Auch die ausnahmslose positive Bewertung von den Neulingen kann nicht falsifiziert werden. Im Bezug auf die Differenzierung zwischen bekannten und unbekannten Orten wird die Interpretationshilfe in Bezug auf Kopenhagen allgemein positiver bewertet. 


\subsubsection{BEANTWORTUNG DER FORSCHUNGSFRAGE}

Aus den Ergebnissen der einzelnen Unterhypothesen kann die Forschungsfrage Ist eine Interpretationshilfe eine geeignete Methode um die Benützung von komplexen interaktiven thematischen Karten zu unterstützen? mit "Ja" beantwortet werden.

Generell stehen die Nutzenden der Hilfe einer Interpretationshilfe positiv gegenüber, da sie die Interpretation der Inhalte vereinfacht und beschleunigt. Vor allem bei Städten, mit denen kaum geographisches Vorwissen verknüpft wird, wird die Interpretationshilfe von den Nutzenden als Unterstützung gesehen. Neben der positiven Bewertung der Nutzenden werden auch objektiv mehr Inhalte korrekt interpretiert. Ihr unterstützender und leitender Einfluss ist vor allem bei Nutzenden mit wenig Erfahrung bei der Verwendung von Karten sowie bei Orten, ohne geographisches Vorwissen, zu sehen. Durch den Einfluss der Interpretationshilfe auf Nutzenden kann von den AutorInnen gesteuert werden, welche Inhalte von den Nutzenden wahrgenommen werden. Die Nutzenden vertrauen vor allem bei schwer zu interpretierenden Inhalten auf die Interpretationshilfe, ohne ihre Aussagen selbst in der KIT-Karte zu überprüfen. Die Interpretationshilfe hat Einfluss auf das Explorieren der Nutzenden in der Karte. Es wird weniger Zeit mit der Karte verbracht sowie einige Bereiche nicht selbstständig angesprochen. Vor allem bei unerfahrenen Nutzenden hält sich die Exploration stärker an die von der Interpretationshilfe vorgegebene Führung.

In Kapitel 5 wird dieses Testergebnis aufgegriffen und hinsichtlich ihrer Vor- und Nachteile im Einsatz diskutiert. Zuvor beschreibt jedoch Kapitel 4.4.5., welche Einschränkungen bei der Gültigkeit der Ergebnisse zu beachten sind.

\subsubsection{EINSCHRÄNKUNGEN UND SIGNIFIKANZ DER TESTERGEBNISSE}

Der Test wurde nach den in Kapitel 3 festgelegten Methoden durchgeführt. In diesem wird bereits hingewiesen, dass auch bei sorgfältiger Auswahl und Anwendung der Methoden auf bestimmte Einschränkungen rücksichtgenommen werden muss. Diesen Einschränkungen müssen bei der Interpretation der Ergebnisse berücksichtigt werden, wodurch die Beantwortung der Forschungsfrage nur unter bestimmten Vorbehalten gültig ist. Im Folgendem werden nun konkrete Punkte aufgelistet, die die Gültigkeit der Ergebnisse beeinflussen.

\section{a) Usability-Test der KIT-Karten}

Wie in Kapitel 3.1 beschrieben, werden Usability-Evaluationen nicht nur summativ durchgeführt, um die Auswirkungen eines Systems zu untersuchen, sondern auch formativ eingesetzt, um ein Produkt auf die Ansprüche der Nutzenden abzustimmen und somit zu verbessern. Bevor die Interpretationshilfe in der KIT-Karte getestet wurde, hätte auch eine intensive Studie über die KIT-Karte selbst durchgeführt werden können, um Probleme bei der Bedienung auszuschließen. Da die Beurteilung der Usability von KIT-Karten nicht Thema dieser Arbeit ist und aufgrund begrenzter zeitlicher Ressourcen nicht durchgeführt werden kann, wurde die KIT-Karte nur in kleinem Rahmen getestet. Zum einen wurde während des Erstellungsprozesses Rücksprache mit zwei ExpertInnen der Kartographie gehalten (Professor und Betreuerin dieser Arbeit), zum anderen wurde im Zuge des Pretests die Verwendung der KIT-Karte beobachtet. Diese Schritte können bereits eine gute Basis bilden, um eine 
nutzerfreundliche Karte zu erstellen und gelten daher als ausreichend für den Test der Interpretationshilfe.

Da diese Herangehensweise nicht alle Unverständlichkeiten und Benutzerprobleme vollständig abdecken kann, ergaben sich im Laufe des Tests Anmerkungen der Testpersonen zur Verwendung der KITKarten. Da auch kleinere Unverständlichkeiten bei der Verwendung einer Karte einen Einfluss auf die Kommunikation der Inhalte haben, sollen die Aspekte, die im Zuge des Usability-Tests über die Interpretationshilfe für die KIT-Karte aufgedeckt wurden, nicht unerwähnt bleiben.

Beispielsweise wird erwähnt, dass für eine genauere Untersuchung des Karteninhalts eine Zoomfunktion hilfreich gewesen wäre (TP 82, 83). Auch wird angeführt, dass Hintergrundinformationen hilfreich wären (TP 76) sowie eine Übersicht über alle statistischen Werte auf einen Blick um die Daten vergleiche zu können (82). Technisch bedingt wurden die Kartenaussagen beim Wechseln zwischen Statistik und Karte und Wien und Kopenhagen auf die Ausgangseinstellung zurückgestellt. Eine Testperson gibt an, dieses Zurückstellen hätte es ihr erschwert Vergleiche zwischen den einzelnen Städten anzustellen (TP 72). Interessante Kommentare waren auch im Bezug auf das Straßennetz und die Bebauung zu vernehmen. Angemerkt wird, dass diese beiden Aussageebenen nur schwer zu erkennen sind und deutlicher dargestellt werden hätte sollen. (TP 73, 67). Allerdings war diesen Testpersonen nicht bewusst, dass man diese auch ohne Choropletenkarten darstellen kann und daher ohne Überdeckung begutachten kann. Das zeigt, dass die Menübedienung zumindest nicht für alle ganz verständlich war.

Im Fall dieses Usability-Tests wurde die KIT-Karte durch einen Prototyp umgesetzt, in dem naturgemäß die Funktionalität eingeschränkt ist. Auch wenn möglicherweise nicht alle Elemente der Karte so funktionieren wie man es von ihnen erwartet, hatten alle Testpersonen dieselben Voraussetzungen. Das Testergebnis wird vermutlich deshalb nicht stark verzerrt.

\section{b) Logik und Vorwissen}

Die Auswahl der Testpersonen und des Inhalts der Karten wurde nach festgelegten Kriterien vorgenommen. Der Eignungstest stellt sicher, dass die Testpersonen alle über ein ähnliches Können und Vorwissen verfügen. Dennoch haben bestimmte Interessen und Aufgabenstellungen im Beruf dazu geführt, dass manche Testpersonen über mehr Wissen verfügten als andere. So meinte beispielsweise eine Expertin zur Wienkarte: "Einkommen und Dichte hab ich sowieso im Kopf" (TP 70). Wie bereits in Kapitel 4.4.4.1 beschrieben hatten auch generelle Annahmen zur Verteilung der Ärzte Einfluss auf die Bewertung der Aussagen. Auch wenn keine der Testpersonen tatsächlich über Vorwissen zur Verteilung der ÄrztInnen in Wien und Kopenhagen verfügte, wurden einige Aussagen durch "Logik" richtig bewertet. Wie an Aussage 5c) zu Kopenhagen zeigt, muss diese Logik allerdings nicht immer richtig sein. Dennoch muss beachtet werden, dass diese die Ergebnisse zu Wien beeinflussen können, da Zusammenhänge diesen logischen Annahmen folgen.

Um diesen Effekt auszuschließen sollte ein Thema gewählt werden, zu dessen einzelnen Komponenten kein Vorwissen besteht. Das Thema sollte daher etwas behandeln, zu dem spezialisierte Personen keinen Bezug haben. Raumrelevante Indikatoren mit denen RaumplanerInnen aufgrund ihrer Ausbildung vermutlich schon konfrontiert wurden, sollten auch vermieden werden. 


\section{c) Beobachtetes Klickverhalten}

Für Hypothese 3 wird das Klickverhalten der Testpersonen untersucht. Es wird festgestellt, welche Bereiche in der Karte zu Wien und Kopenhagen selbstständig aufgerufen werden, obwohl sie von der Interpretationshilfe nicht angesprochen werden. Dabei ergeben sich unterschiedliche Ergebnisse für die einzelnen Bereiche in den Karten für Wien und Kopenhagen.

Es kann der Fall auftreten, dass wenn man eine Karte zum ersten Mal sieht, man sich zuerst mit ihren Möglichkeiten und Funktionen vertraut macht. Bei dieser KIT-Karte wurde als erste Karte die Situation in Kopenhagen präsentiert. Es könnte daher sein, dass in dieser Karte Klicks erfolgten, die nicht unbedingt dem Interesse dem Inhalt gegenüber galten, sondern um die KIT-Karte mit ihren Funktionen kennenzulernen. In der Karte zu Wien ist das dann nicht mehr notwendig, weil die Funktionen und der Aufbau der Karte unverändert bleiben. Die Unterscheidung von Wien und Kopenhagen hinsichtlich der aufgerufenen Bereiche ist unter Bedenken dieses Umstandes zu interpretieren.

Weiters kann das erhobene Klickverhalten zu den aufgerufenen Bereichen nur bedingt mit der tatsächlichen Exploration der Inhalte in Zusammenhang gesetzt werden. Über Zählen, welche Bereiche von den Testpersonen aufgerufen wurden, kann nicht direkt darauf geschlossen werden, wie intensiv die Inhalte der Karte studiert werden. Im Gegensatz steht hier das schnelle Durchklicken und Aufrufen von vielen Informationen ohne den Inhalt dabei (genau) zu betrachten mit dem weniger, aber doch gezielten Zusammenstellen von Karten und Aussageebenen, die den Inhalt effizient erfassen lassen.

Um die Ergebnisse über das Klickverhalten aussagekräftiger zu machen, könnten die Testgruppen nochmals weiter unterteilt werden. Weitere Szenarien werden entwickelt, die noch zusätzlich einen Wechsel zwischen Wien und Kopenhagen als zuerst präsentierter Karte enthalten. Weiter könnte das Interaktionsverhalten nach genaueren Gesichtspunkten betrachtet werden. Für jede Aussageebene der Karte sollte festgestellt werden, wie lange diese aufgerufen ist. Diese Untersuchung könnte weiter mit der Eye-Tracking-Methode kombiniert werden. Sie stellt sicher, dass den aufgerufenen Inhalten auch tatsächlich Aufmerksamkeit geschenkt wird.

\section{d) empfundener Zeitdruck}

Wie in Kapitel 3.1. beschrieben wird durch die Durchführung eines Usability-Tests eine künstliche Situation geschaffen. Auch bei diesem Test empfanden sich manche Testpersonen einer "Testsituation" ausgesetzt. Vor allem der empfundene Zeitdruck spielte hier eine Rolle. Für die Verwendung der Karte wurde ein Zeitraum von 20 Minuten angesetzt. Stößt man bspw. auf einer Website auf eine interaktive Karte, wird, wie in Kapitel 2.3.4.4. beschrieben, vermutlich nicht viel Zeit für die Erfassung des Inhalts aufgewendet. 20 Minuten wurde demnach im Testdesign als lange Zeitspanne angenommen, die die Testpersonen für die Informationsaufnahme eigentlich nicht vollständig beanspruchen sollten. Dennoch haben 20 \% der Personen die Zeit vollständig ausgeschöpft. Die Hälfte davon gab an, sich unter Zeitdruck gesetzt gefühlt zu haben. Sie beschreiben die vorgegebene Situation für sie als ungewohnt:

"Ich brauche für Recherchen mehr Zeit, weil ich alles genau machen möchte. 20 Minuten sind für mich wischiwaschi. Wenn du was gescheit machen willst, musst du dir die Zeit nehmen." (TP 85) 
"Wenn ich Zeitdruck habe kann ich mich nicht so gut fokussieren. Wenn ich mich so hinsetzen würde, würde ich mir länger Zeit nehmen. Vielleicht würde ich nicht mehr Zeit brauchen, aber ich hätte keinen Druck und eine konkretere Fragestellung." (TP 69)

Um das Gefühl, unter zeitlichem Druck zu stehen, zu minimieren, könnte die Zeitspanne größer gewählt werden. Da eine zeitliche Vorgabe die Organisation eines Tests jedoch erleichtert, kann dieser Effekt nur schwer vollkommen aufgehoben werden.

\section{e) unklare Aufgabenstellungen}

Bei der Aufgabenstellung in Frage 4 des Usability-Tests wurden die Testpersonen aufgefordert, die KIT-Karte nach bestimmten Gesichtspunkten zu untersuchen. Obwohl bei der Formulierung auf eine verständliche und vollständige Beschreibung geachtet wurde, war den Testpersonen nicht immer ganz klar was zu tun ist. Eine der Testpersonen weist darauf hin, dass "konkretere Fragen" (TP 72) es leichter gemacht hätte, die Inhalte zu untersuchen. Vor allem ginge aus der Aufgabenstellung nicht hervor, dass die Verteilung der Arztpraxen nicht nur mit den erwähnten Inhalten zu Einkommen, Wohnungspreisen und Bevölkerungsdichte in Bezug zu setzen sind, sondern auch mit dem Verlauf der Straßen und der Bebauung (TP 72). Diese wird nur durch den Hinweis zu "[..] den weiteren in der Karte enthaltenen Informationen" angedeutet. Obwohl diese Anmerkungen nachzuvollziehen sind, wurde die Aufgabenstellung absichtlich in Bezug auf diese beiden Aufgabenstellungen etwas offener gestellt, um die selbstständige Exploration der Testteilnehmenden zu untersuchen.

Weiters war einigen Testpersonen in Szenario 1 die verpflichtende Verwendung der Interpretationshilfe nicht ganz bewusst. Obwohl in der Aufgabenstellung durch den Hinweis "In der nun folgenden Karte finden Sie/findest du rechts neben der Kartendarstellung weitere Anweisungen, die dich anleiten. Bitte folge diesen über den "Weiter"-Button." eindeutig auf ihre Verwendung hingewiesen wird, wurde das von einigen Testpersonen nicht umgesetzt. Fast die Hälfte der Testpersonen in Szenario 1, haben, vor Verwendung der Interpretationshilfe, die Karte selbstständig studiert. Bei absoluter NichtBeachtung der Interpretationshilfe wurde, solange bei der Zuteilung der Testpersonen zum Szenario noch Spielraum bestand, jene Testpersonen Szenario 2 zugeordnet. Im Laufe der weiteren Tests wurde allerdings nach 5 Minuten die Aufgabe abgebrochen, die Gründe für die Missachtung der Interpretationshilfe erfragt und die Aufgabe mit Verwendung der Interpretationshilfe neu gestartet. Einerseits wurde diese beispielsweise absichtlich nicht (gleich) verwendet. Eine Expertin begründet das damit, dass sie "zuerst selber mal schauen wollte was es so gibt" (TP 75) bevor sie die Führung der Interpretationshilfe in Anspruch nimmt. Andererseits wurde die Interpretationshilfe schlichtweg "übersehen" (TP 87) und nicht wahrgenommen, da die Testpersonen "schon so auf die Inhalte konzentriert" (TP 76) waren.

Gespräche mit den Testpersonen zeigten, dass die Aufgabenstellung mit einer hohen Dichte an Informationen als recht lang empfunden wird. Nicht alle Anweisungen könnten sich dabei genau gemerkt werden. Um diesem entgegenzuwirken, könnten nach dem selbstständigen Lesen der Aufgabenstellung durch die Testpersonen, die wichtigsten Punkte vom Testleitenden nochmals mündlich zusammengefasst werden. So kann sichergestellt werden, dass die Aufgabe auch korrekt von den Testpersonen verstanden wird. Um die Testpersonen weiters nicht dazu zu verleiten, die Interpretationshilfe zu übersehen, könnte diese noch etwas prominenter in der KIT- Karte platziert werden. Obwohl sie der einzige Fließtext in der Karte ist, scheint sie sich nicht besonders gegenüber den anderen Karteninhalten abzuheben. Sie könnte beispielweise im ersten Schritt für die Begrüßung über 
der Karte präsentiert werden und erst später neben die Karte platziert werden. Weiters könnte sie durch einen farblichen Rahmen und eine andere Hintergrundfarbe deutlicher gestaltet werden.

\section{f) Signifikanz der Testergebnisse}

Wie bereits in Kapitel 3.2.4 beschrieben, zielt das Ergebnis dieser Arbeit nicht auf einen großen Testumfang ab, sondern es sollen vielmehr erste Einblicke in die Fragestellung gewonnen werden. Aufgrund des eher kleinen Testumfangs wird im Rahmen dieser Arbeit auf eine Signifikanzprüfung verzichtet. Bei der Interpretation der Ergebnisse aus Kapitel 4.4.4 muss daher berücksichtigt werden, dass nicht überprüft ist, ob die gewonnen Erkenntnisse auf die Grundgesamtheit der Menschheit verallgemeinert werden können. Um umfangreichere Ergebnisse zu dieser Thematik zu erhalten, sollten im Zuge weiterer Forschung Tests mit größeren Testgruppen durchgeführt werden. Die gewonnenen Ergebnisse sollten dann weitere Folge auch auf ihre Signifikanz geprüft werden.

Nachdem in diesem Kapitel die Beantwortung der Forschungsfrage hergeleitet wurde und die Gültigkeit der Ergebnisse diskutiert wurde, beschreibt Kapitel 5 nun genauer, welche Auswirkungen beim Einsatz einer Interpretationshilfe zu beachten sind. 


\section{RESÜMEE}

Dieses Kapitel fasst die wesentlichen Punkte dieser Arbeit zusammen und zieht, basierend auf den Testergebnissen des Usability-Test, ein Fazit über den Einsatz von Interpretationshilfen. Die Beurteilung der Interpretationshilfe geschieht in Hinblick auf eine kritische Begutachtung der Implementierung von Interpretationshilfe in KIT-Karten sowie auf die Portierbarkeit der Ergebnisse. Abschließend wird ein Ausblick in zukünftige Forschung gegeben.

\subsection{ZUSAMMENFASSUNG ÜBER DIE "INTERPRETATIONSHILFE"}

Um den Einfluss einer Interpretationshilfe auf die Benützung von interaktiven thematischen Karten zu untersuchen sind unterschiedliche Aspekte zu berücksichtigen. In der Informationsüberbringung, die das allgemeine Ziel einer Karte ist, sind Einflüsse, die durch das Kommunikationsmodell bestimmt sind, sowie kognitive Aspekte, als auch der Zugang über den Inhalte und zusätzliche Informationen präsentiert werden, bestimmend.

Generell ist die Überbringung jeglicher Art von Informationen Störungen ausgesetzt. Aufgrund von verschiedenen Einflüssen kann die ursprünglich von Sender vorgesehene Information beim Empfänger modifiziert eintreffen. Bei der Informationsübertragung durch Kartendarstellungen stellen weiters besonders kognitive Aspekte einen wichtigen Einflussfaktor dar. Durch perzeptive Regeln, Abläufe im Gedächtnis, Aufmerksamkeitsverhalten und Denkvorgänge zum Bedeutungsgewinn wird das 'Lesen' von Karten auf unterschiedliche Weise beeinflusst. Um auch anspruchsvolle Informationen wie sie durch komplexe interaktive thematische Karten dargestellt werden, effizient kommunizieren zu können, wird eine Interpretationshilfe eingesetzt. Sie soll die Kommunikation von Informationen unterstützen, die aufgrund der Einschränkungen bezüglich kommunikativer Grundsätze und den beschränkten kognitiven Ressourcen des Menschen erschwert ist.

Um die Wirksamkeit einer Interpretationshilfe zu prüfen, wird sie mit bestehenden Konzepten im Kontext der Benutzersteuerung in Kartenprodukten in Zusammenhang gesetzt und untersucht. Diese werden hinsichtlich ihrer Ausgewogenheit zwischen Nutzer- und den autorengesteuerter Konzeption diskutiert. Im weiteren Hinblick auf allgemeine Hilfestellungen in Softwareprodukten werden verschiedene Möglichkeiten diskutiert, wie Unterstützung Hilfe und den Nutzenden verfügbar gemacht werden können. Daraus wird ersichtlich, dass die unterstützende Wirkung von Hilfestellungen unter anderem stark davon abhängt, wie diese den Nutzenden präsentiert werden.

Hinsichtlich der kognitiven Aspekte und Nutzerunterstützung kann die Interpretationshilfe als hilfreiche Methode in der Benützung von komplexen interaktiven thematischen Karten gesehen werden. Allerdings wird durch die empirische Evaluation gezeigt, dass der Einsatz einer Interpretationshilfe in diesen Karten mit Vor- und Nachteilen verbunden ist. Zum einen kann die Interpretationshilfe als eine wirksame Methode gesehen werden, die die Nutzenden bei der Verwendung einer KIT-Karte zu den für die Fragestellung wichtigen Informationen hinleitet. Die Nutzenden werden gezielt auf bestimmte Inhalte hingewiesen, wodurch bestimmte Informationen aufgenommen werden. Bei einer 
kurzen Aufmerksamkeitsspanne und kognitiver Überlastung kann sicherstellt werden, dass gewisse Informationen Nutzenden präsentiert werden. Diesen wird dadurch abgenommen, selbst ein Verständnis aus der Karte abzuleiten. Die selbstständige Interpretation und Beschäftigung mit den Inhalten der Karte wird dadurch jedoch eingeschränkt. Es werden überwiegend jene Informationen von den Nutzenden aufgenommen, die in der Interpretationshilfe enthalten sind und demnach von den Kartenautoren vorgesehen sind. Diesem Umstand muss bei ihrem Einsatz Beachtung geschenkt werden.

\subsection{PORTIERBARKEIT UND ANWENDBARKEIT DER ERGEBNISSE}

Das Wissen über den Einsatz von Interpretationshilfen auf die Benützung von Karten ist sowohl für Kartenersteller als auch für Kartennutzende von Bedeutung. Kartenersteller können die Ergebnisse dafür nutzen, um Karten zu erstellen deren Inhalte für die Nutzenden leicht und schnell verständlich sind. Für die Nutzenden ist vor allem der Einblick in die negativen Auswirkungen relevant. Die Ergebnisse ermöglichen es, dass bei den Kartennutzende ein Bewusstsein darüber geschaffen werden kann, wie der Wissensgewissen bei der Verwendung einer Karte beeinflusst wird. Sie können bei der Verwendung von Karten besser darauf achten, nicht alles unhinterfragt hinzunehmen, was von der Interpretationshilfe vorgegeben und präsentiert wird. Durch das Wissen über den Einfluss von bestimmten Methoden wie die Interpretationshilfe eine ist, sollen die Nutzenden dazu angehalten werden, sich selbstständig ein Bild über die dargestellten räumlichen Daten zu machen. Die Ergebnisse zur Untersuchung der Interpretationshilfe können weiters auch für Bereiche abseits der Kartographie angewendet werden. Werden komplexe thematische interaktive Karten als Softwareprodukte gesehen, können in Hinblick auf die Nutzersteuerung auch Informationen für andere Anwendungen abgeleitet werden.

\section{Relevanz für die Raumplanung}

Auch für Aufgabenstellungen in der Raumplanung ist der Einfluss der Interpretationshilfe relevant. Für raum(planungs)bezogene Fragestellungen werden häufig thematische Karten für verschiedene Zwecke eingesetzt. Sie dienen u.a. zum Erkenntnisgewinn und zur Entscheidungsfindung bei Planung und Umsetzung als auch für die Kommunikation innerhalb des Planungsteams sowie an Planungsbetroffene. Die Interpretationshilfe kann dabei vielfältig Einsatz finden. Beispielweise kann sie Planinhalte zum Erkenntnisgewinn beschreiben und damit auf die Entscheidungsfindung Einfluss nehmen (siehe Kapitel 4.4.4.1). Auch bei der Kommunikation zwischen Planenden und Planungsbetroffenen kann sie eingesetzt werden. Unerfahrenen Beteiligten wird durch die Interpretationshilfe ermöglicht, die Inhalte einer komplexen thematischen Karte besser zu verstehen (siehe Kapitel 4.4.4.1). Die Interpretationshilfe kann hierbei als Instrument eingesetzt werden, das dazu dienen könnte die Planungsbetroffenen für die Teilnahme am Planungsprozess zu aktivieren und motivieren. Missbräuchlich eingesetzt könnte die Interpretationshilfe auch hier bestimmte Meinungen Richtungen vorgeben.

\subsection{KRITIK AM EINSATZ VON INTERPRETATIONSHILFEN}

Wie bereits unter Punkt 5.1 beschrieben gehen mit dem Einsatz einer Interpretationshilfe viele Vorteile einher. Für die Kartennutzenden wird durch diese Methode eine Möglichkeit geschaffen, die die 
Benützung von komplexen interaktiven thematischen Karten unterstützt und erleichtert. Die positive Unterstützung birgt allerdings auch negative Seiten. Bei der Implementierung einer Interpretationshilfe wird die selbstständige Beschäftigung mit der Karte eingeschränkt und eine vorgegebene Leitung zur Benützung erkennbar. Ihr Einsatz ist daher mit einer großen Verantwortung an die Kartenersteller verbunden.

Generell ist der gesamte Erstellungsprozess einer Karte durch Entscheidungen der Kartenautoren geprägt. Beispielsweise kann durch die Wahl des Kartenausschnitts, der Symbologie oder der Generalisierung Einfluss darauf genommen werden, was die Karte an die Kartennutzenden vermittelt (Monmonier 1996). Auch die für die Interpretationshilfe getroffenen Entscheidungen zu ihren Inhalten sind dabei ausschlaggebend. Kommunizierte Zusammenhänge sowie verwendete Wörter können Auswirkungen auf die Interpretation der Nutzenden haben (siehe Kapitel 4.4.4.1). Wird der Einfluss der Interpretationshilfe auf die Karteninterpretation nicht wohlwollend genutzt sondern durch unehrliche Interessen missbraucht, können Tatsachen verschleiert oder manipuliert werden. Nutzende können dabei zu einer bestimmten Interpretation 'gedrängt' werden. Dies ist insbesondere bei Nutzenden mit wenig Erfahrung im Kartenlesen von Bedeutung, die noch stärker auf die Inhalte der Interpretationshilfe vertrauen (siehe Kapitel 4.4.4.1). Informationen, die vorgefassten Meinungen entsprechen oder Begriffe, die bereits konnotiert sind, können ein Manipulationspotential bergen.

Monmonier (1996) vertritt in Bezug auf das 'Lügen mit Karten' folgende Meinung: "Eine einzelne Karte stellt nur eine von unendlich vielen Möglichkeiten dar, einen bestimmten Sachverhalt oder bestimmte Daten kartographisch wiederzugeben" (Monmonier 1996, 14). Diese Aussage hat auch große Relevanz für die Inhalte einer Interpretationshilfe. Die enthaltene Beschreibung mit den verwendeten Wörtern ist nur eine von vielen Möglichkeiten, die aufgrund eines bestimmten Interesses oder Zieles vom Kartenautor ausgewählt wurde.

\subsection{AUSBLICK IN WEITERE FORSCHUNG}

Das konkrete Ergebnis zur Bewertung der Interpretationshilfe ist auf die konkrete Testumsetzung der KIT-Karte und die gewählte Implementierung der Interpretationshilfe beschränkt. Die Komplexität des Aufbaues der KIT-Karte stellt unterschiedliche Ansprüche an eine Nutzerführung. Wird die Interpretationshilfe nach anderen Grundsätzen umgesetzt, könnte möglicherweise ein anderer Einfluss auf die Verwendung von komplexen interaktiven thematischen Karten abgeleitet werden. Veränderte Ergebnisse sind vermutlich dann zu erwarten, wenn die Integration der Interpretationshilfe in die Karte (bspw. statt neben der Karte darüber liegend) oder die verpflichtende Verwendung (bspw. freie Wahl zwischen Verwendung oder selbstständiger Exploration) verändert wird. Dieser Umstand gibt Anlass, den Einfluss von Interpretationshilfen im Zuge weiterer Forschung zu untersuchen. Weiters kann die methodische Herangehensweise in zukünftige Forschungen erweitert werden. In diesem Zusammenhang kann der Einfluss der Interpretationshilfe auf das Richten der Aufmerksamkeit durch Eye Tracking-Methoden untersucht werden. Weiters könnten detaillierte Beobachtungen zur Explorationen der Karteninhalte unternommen werden, um gezieltere Aussagen zum Einfluss der Interpretationshilfe machen zu können. Auch der Einfluss auf die Interpretation hinsichtlich Wortwahl, Formulierungen und einseitiger Interpretation von räumlichen Zusammenhängen sollte im Zuge weiterer Forschung untersucht werden. 


\section{QUELLEN}

\section{Online Quellen zuletzt abgerufen und geprüft am 31.1.2015}

AirFastTickets. 2014. „AirFastTickets | Günstige Flugtickets, Günstige Hotels, Mietwagen“. http://www.airfasttickets.de/.

Alliance for Technology Access. 2004. Computer Resources for People with Disabilities: A Guide to Assistive Technologies, Tools and Resources for People of All Ages. Hunter House.

anagraphic. 2014. „anaptár 2012“. anagraphic. http://anagraphic.hu/en/anaptar-2012/.

Baars, B.J. 1988. A cognitive theory of consciousness. Cambridge: Cambridge University.

Beetz, J. 2013. 1+1=10: Mathematik für Höhlenmenschen. Springer Berlin Heidelberg..

Benway, J.P. 1998. „Banner blindness: The irony of attention grabbing on the World Wide Web“. In Proceedings of the Human Factors and Ergonomics Society Annual Meeting, 42:463-67. SAGE Publications.

Blades, M. und C. Spencer. 1986. „The implications of psychological theory and methodology for cognitive cartography". Cartographica: The International Journal for Geographic Information and Geovisualization 23 (4): 1-13.

Bräuer, H. 2006. Poppers Falsifikationismus - Vermutungen und Widerlegungen. Einführung in die Theoretische Philosophie. Technische Universität Dresden.

Bucher, H.-J., und P. Schumacher. 2006. „The relevance of attention for selecting news content. An eye-tracking study on attention patterns in the reception of print and online media". Communications 31 (3): 347-68.

Cammack, R. G. 1999 „New map design challenges: Interactive map products for the World Wide Web." In Multimedia Cartography: 155-172. Springer Berlin Heidelberg..

Carr, N. 2011. The shallows: What the Internet is doing to our brains. New York \& London: WW Norton \& Company.

Cartwright, W. 1999. „Extending the map metaphor using web delivered multimedia“. International Journal of Geographical Information Science 13 (4): 335-53.

Cartwright, W. 2004. „Engineered serendipity: Thoughts on the design of conglomerate GIS and geographical new media artifacts". Transactions in GIS 8 (1): 1-12.

Cockburn, A., und B. McKenzie. 2001. „What do Web users do? An empirical analysis of Web use“. International Journal of human-computer studies 54 (6): 903-22.

Cooper, A., R. Reimann, und D. Cronin. 2007. About Face 3: The Essentials of Interaction Design. Wiley.

Corbin, M. 2003. „From online help to embedded user assistance“. In ANNUAL CONFERENCE-SOCIETY FOR TECHNICAL COMMUNICATION, 50:295-98. Citeseer. 
Dix, A., und G. Ellis. 1998. „Starting simple: adding value to static visualisation through simple interaction". In Proceedings of the working conference on Advanced visual interfaces, 124-34. L'Aquila, Italy: ACM.

Duffy, T. M., J. E. Palmer, und B. Mehlenbacher. 1992. Online Help: Design and Evaluation. Ablex Pub.

Elzakker, C.J.M. van, und A. L. Griffin. 2013. „Focus on Geoinformation Users“. GIM International 27 (8): online.

Faulkner, L.. 2003. „Beyond the five-user assumption: Benefits of increased sample sizes in usability testing". Behavior Research Methods, Instruments, \& Computers 35 (3): 379-83.

Findlay, J. M., R. Walker, und R.W. Kentridge. 1995. Eye movement research: Mechanisms, processes and applications. Bd. 6. Elsevier.

Fleckenstein, L. 1991. „How Maps Lie“. Syracuse University Magazine 8 (1): 7.

Freedman, E. G., und P. Shah. 2002. „Toward a model of knowledge-based graph comprehension“. In Diagrammatic representation and inference, 18-30. Springer.

Galitz, W. O. 2007. The Essential Guide to User Interface Design: An Introduction to GUI Design Principles and Techniques. Wiley.

Gartner, G. 2000. „Karten im Internet“. Deutsche Gesellschaft für Kartographie e.V., Kartographische Schriften, 4: 43-49.

Gediga, G., und K. C. Hamborg. 2002. „Evaluation in der Software-Ergonomie“. Zeitschrift für Psychologie/Journal of Psychology 210 (1): 40-57.

Gouin, D., V. Lavigne, und A. Bergeron-Guyard. 2012. „Human-computer interaction with an intelligence virtual analyst". Proceedings of Knowledge Systems for Coalition Operations, IHMC, Pensacola, FL.

Hake, G., D. Grünreich, und L. Meng. 2002. Kartographie: Visualisierung raum-zeitlicher Informationen. de Gruyter.

Hall, L. 2006. „Performance Support: Online Help and Advisors“. In International Encyclopedia of Ergonomics and Human Factors, herausgegeben von W. Karwowski, 2. Auflage. Taylor \& Francis.

Harrower, M. 2007. „The cognitive limits of animated maps“. Cartographica: The International Journal for Geographic Information and Geovisualization 42 (4): 349-57.

Hedberg, J. G., und B. Harper. 1992. „Creating interface metaphors for interactive multimedia“. In Proceedings of the International Interactive Multimedia Symposium, 27-31.

Hegarty, M., M. Canham, und S. I. Fabrikant. 2010. „Thinking about the weather: How display salience and knowledge affect performance in a graphic inference task." Journal of Experimental Psychology: Learning, Memory, and Cognition 36 (1): 37.

Herrmann, D., und L. W. Pickle. 1996. „A cognitive subtask model of statistical map reading“. Visual Cognition 3 (2): 165-90.

Hoffmann, M. 2001. „Peirces Zeichenbegriff: seine Funktionen, seine phänomenologische Grundlegung und seine Differenzierung“. 
Hullman, J., und N. Diakopoulos. 2011. „Visualization rhetoric: Framing effects in narrative visualization“. Visualization and Computer Graphics, IEEE Transactions on 17 (12): 2231-40.

Iliinsky, N., und J. Steele. 2011. Designing Data Visualizations: Representing Informational Relationships. O’Reilly Media.

Imhof, E. 1972. Thematische Kartographie. De Gruyter.

Intemann, F. 2002. Kommunikation - Hypertext - Design. Waxmann.

Issmael, L.S., und P.M.L. Menezes. 2007. „Cognitive Cartography: An Instrument to become spatial of geographic Information“. In International Cartographic Conference. Bd. 23. Moscow.

Kalbach, J. 2008. Handbuch der Webnavigation. O’Reilly.

Kearsley, G. 1988. Online Help Systems: Design and Implementation. Ablex Publishing Corporation.

Keyes, J. 2002. Software Engineering Handbook. Taylor \& Francis.

Kelnhofer, F. 2000. „Interaktive Karten (Atlanten) und Multimedia-Applikationen“. In Geowissenschaftliche Mitteilungen, H. 53, Technische Universität Wien.

Koláčný, A. 1969. „Cartographic information - a fundamental concept and term in modern cartography". The cartographic journal 6 (1): 47-49.

Kraak, M. J., und A. Brown. 2001. Web Cartography: Developments and Prospects. Third Edition. London: Taylor \& Francis.

Kraak, M. J., und F. Ormeling. 2010. Cartography: Visualization of Spatial Data. Third Edition. Taylor \& Francis.

Laufmann, S.C. 1998. Agent Technology: Foundations, Applications, and Markets. Herausgegeben von $\mathrm{N}$. Jennings und M. J. Wooldridge. Springer.

Lehrstuhl Kartographie. 2004. „Kartographische Zeichentheorie“. In Visualisierung von GISLehrstoffen - Modul 10. Universität Bonn - Insitut für Kartographie und Geoinformation.

Lexikon der Kartographie und Geomatik. 2014. „kartographische Informationsverarbeitung“. Spektrum.de. Zugegriffen Juli 11. http://www.spektrum.de/lexikon/kartographiegeomatik/kartographische-informationsverarbeitung/2719.

MacEachren, A. M. 2004. How Maps Work: Representation, Visualization, and Design. Guilford Press.

Matlin, M. W. 2002. Cognition. Harcourt College Publishers.

media.org. 2000. „A Shared Reality“. Mappa.Mundi Magazine. http://mappa.mundi.net/cartography/Maps/.

Monmonier, M. S. 2011. „Reflection Essay: Strategies for the Visualization of Geographic Time-Series Data". In Classics in Cartography: Reflections on Influential Articles from Cartographica, herausgegeben von M. Dodge. Chichester, UK: John Wiley \& Sons, Ltd.

Montello, D. R. 2002. „Cognitive map-design research in the twentieth century: Theoretical and empirical approaches. Cartography and Geographic“. Information Science 29 (3): 283-304. 
Montello, D.R. 2009. „Cognitive research in GIScience: Recent achievements and future prospects“. Geography Compass 3 (5): 1824-40.

Naone, E. 2009. „TR10: Intelligent Software Assistant“. Mar.-Apr.

NCGIA. 1997. „Moving Beyond the Map as Metaphor: Representation and multiple realities“. In Workshop on Geographies of the Information Society. Upham Hotel, Santa Barbara, CA.

Neowin. 2014. „Clippy lives! Microsoft's animated Office Assistant returns“. Neowin. Zugegriffen November 18. http://www.neowin.net/news/clippy-lives-microsofts-animated-officeassistant-returns.

Nielsen, J. 1997. „How users read on the Web“. Aufgerufen über Internet: http://www.nngroup.com/articles/how-users-read-on-the-web/ [Datum 29.09. 2014]. Nielson Norman Group.

Olbrich, G., M. Quick, und P. D. J. Schweikart. 2014. Desktop Mapping: Grundlagen und Praxis in Kartographie und GIS. Springer Berlin Heidelberg.

Ooms, K. 2012. „Maps, how do users see them? - An in depth investigation of the map users' cognitive processes". Ghent University.

Peterson, M. P., und C. Sawyer, Hrsg. 2008. International Perspectives on Maps and the Internet. Springer Berlin Heidelberg.

Platt, Charles. 1995. „Interactive Entertainment: Who Writes It? Who Reads It? Who Needs It“. Wired 3: 144-49.

Popper, K. R. 1935. Logik der forschung: zur erkenntnistheorie der modernen naturwissenschaft. J. Springer Berlin Heidelberg.

Raskin, J. 2000. The Humane Interface: New Directions for Designing Interactive Systems. AddisonWesley.

Rensink, R. A. 2002. „Change detection“. Annual review of psychology 53 (1): 245-77.

Rogers, Y., H. Sharp und J. Preece. 2011. Interaction Design: Beyond Human - Computer Interaction. Wiley.

Roth, R. E. 2013. „Interactive maps: What we know and what we need to know“. Journal of Spatial Information Science Number 6: 59-115.

Rubin, J., D. Chisnell, und J. Spool. 2008. Handbook of Usability Testing: How to Plan, Design, and Conduct Effective Tests. Wiley.

Sarodnick, F., und H. Brau. 2011. Methoden der Usability Evaluation: wissenschaftliche Grundlagen und praktische Anwendung. Huber.

Schertenleib, M. H., und H. Egli-Brož. 2008. Grundlagen Geografie: Aufgaben des Fachs, Erde als Himmelskörper und Kartografie: Lerntext, Aufgaben mit Lösungen und Kurztheorie. Compendio Bildungsmedien.

Segel, E., und J. Heer. 2010. „Narrative visualization: Telling stories with data“. Visualization and Computer Graphics, IEEE Transactions on 16 (6): 1139-48. 
Shannon, C. E., und W. Weaver. 1949. The mathematical theory of communication (Urbana, IL. University of Illinois Press IL.

Silver, M. 2004. Exploring Interface Design. Cengage Learning.

Simons, D. J. 2000. „Attentional capture and inattentional blindness“. Trends in cognitive sciences 4 (4): 147-55.

Simons, D. J., und D. T. Levin. 1998. „Failure to detect changes to people during a real-world interaction“. Psychonomic Bulletin \& Review 5 (4): 644-49.

Slocum, T. A., C. Blok, B. Jiang, A. Koussoulakou, D. R. Montello, S. Fuhrmann, und N. R. Hedley. 2001. "Cognitive and usability issues in geovisualization". Cartography and Geographic Information Science 28 (1): 61-75.

Smiciklas, M. 2012. The Power of Infographics: Using Pictures to Communicate and Connect with Your Audiences. Que Pub.

University of Zurich. 2014. „Thematische Kartographie: Definition“. GITTA - Geographic Information Technology Training Alliance. Zugegriffen Oktober 8. http://www.gitta.info/website/en/html/index.html.

Weinreich, H., H. Obendorf, E. Herder, und Matthias Mayer. 2008. „Not quite the average: An empirical study of Web use". ACM Transactions on the Web (TWEB) 2 (1): 5.

Wolfe, Jeremy M. 1994. "Guided search 2.0 a revised model of visual search“. Psychonomic bulletin \& review 1 (2): 202-38.

Ye, Y. 2001. Human-computer Interaction: INTERACT'01 : IFIP TC.13 International Conference on Human-Computer Interaction, 9th-13th July 2001, Tokyo, Japan. Herausgegeben von M. Hirose. IOS Press.

ZEIT ONLINE. 2014. „Gesundheitswesen: Wie die Medizin dem Geld folgt“. ZEIT ONLINE. http://www.zeit.de/wirtschaft/2014-04/arzt-facharzt-praxis-verteilung-berlin-hamburgkoeln-muenchen-interaktiv\#stadtteile/kaufkraft/hamburg. 


\section{ABBILDUNGSVERZEICHNIS}

Abbildung 1-1 Beispiel einer KIT-Karte: Ausschnitt aus ZEIT ONLINE Karte "Geld steuert Ärzte" ............... 12

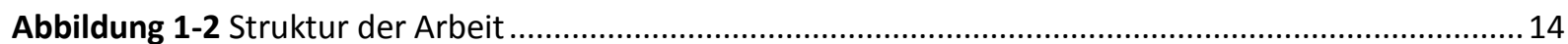

Abbildung 2-1 Begrenze Leistung des Gehirns für Lesen und Verstehen von komplexen Visualisierungen 17

Abbildung 2-2 Unterschied zwischen Infographik und Datenvisualisierung ............................................. 19

Abbildung 2-3 Beispiel einer Datenvisualisierung mit einer großen Menge an Daten ................................20

Abbildung 2-4 Beispiel einer Infographik 'Medical Health Care' ............................................................. 21

Abbildung 2-5 Kommunikationssystem nach Shannon und Weaver.....................................................22

Abbildung 2-6 Kartographie als Kommunikationsprozess von Informationen.......................................... 23

Abbildung 2-7 Modell der kartographischen Kommunikation nach Kolácný (1969) .................................24

Abbildung 2-8 Die Gestaltgesetze nach Wertheimer und Köhler............................................................2 27

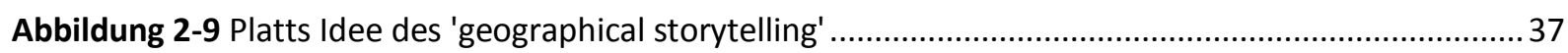

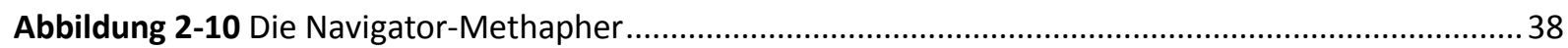

Abbildung 2-11 Beispiel zur Umsetzung von sequentieller und visueller Navigation ................................39

Abbildung 2-12 Das von Monmonier entwickelte Konzept der 'Graphic Scripts' ....................................40

Abbildung 2-13 Der Serendipity-Ansatz führt zu zufälligen Entdeckungen........................................... 41

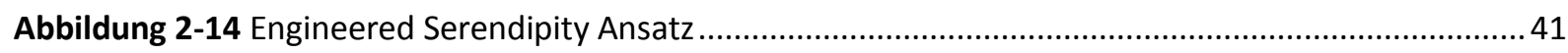

Abbildung 2-15 Die Martiniglas-Struktur als autorengesteuerte Methode ............................................43

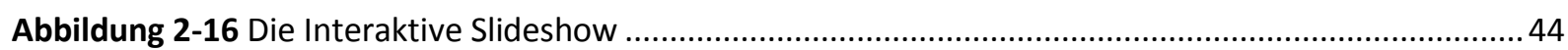

Abbildung 2-17 Die Drill Down Story als nutzergesteuerter Zugang .....................................................44

Abbildung 2-18 Tooltip in Microsoft Office Word 2007 bei aktiver Maus ...................................................49

Abbildung 2-19 Assistent 'Clippy' und seine Kollegen aus Microsoft Office 1997-2003 .............................50

Abbildung 2-20 Dialog in einem Online-Buchungssystem für Flugreisen................................................5 51

Abbildung 2-21 Wizard für Untersützung einer Softwareinstallierung ....................................................52

Abbildung 4-1 ZEIT ONLINE Karte mit aktivierter Tour zur Unterstützung der Kartennutzenden...............70

Abbildung 4-2 Inhalte der KIT-Karte am Beispiel Kopenhagen .................................................................. 72

Abbildung 4-3 Implementierung der Interpretationshilfe im Interface KIT-Karte ...................................74

Abbildung 4-4 KIT-Karte mit Interpretationshilfe und aktiver Mouse-Over-Funktion ................................75

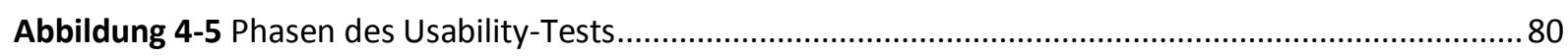

Abbildung 4-6 Häufigkeiten zur Verwendung von KIT-Karten von Neulingen und ExpertInnen .................88

Abbildung 4-7 Einschätzung der Neulinge über die Verwendung von KIT-Karten ....................................89

Abbildung 4-8 Einschätzung der ExpertInnen über die Verwendung von KIT-Karten.................................90

Abbildung 4-9 Verteilung der Arztpraxen in Kopenhagen in Bezug auf das.............................................93

Abbildung 4-10 Verteilung der Arztpraxen Wien in Bezug auf das Straßennetz........................................94

Abbildung 4-11 Richtige Antworten in Szenario 1 und Szenario 2 nach Testgruppen................................97

Abbildung 4-12 Richtige Antworten zu Kopenhagen ..............................................................................98

Abbildung 4-13 Aufgebrachte Zeit zur Verwendung der Karte in Szenario 1 und Szenario 2....................100

Abbildung 4-14 Aufgebrachte Zeit zur Verwendung der Karte nach Szenario.......................................... 101

Abbildung 4-15 Einschätzung des Schwierigkeitsgrad zum Lösen der Aufgaben........................................104

Abbildung 4-16 Einschätzung über die Verwendung von KIT-Karten mit Interpretationshilfe .................105

Abbildung 4-17 Subjektives Empfinden bei der Aufgabenlösung..........................................................106

Abbildung 4-18 Bewertungen der Interpretationshilfe hinsichtlich bekannter und unbekannter Orte ....108 


\section{TABELLENVERZEICHNIS}

Tabelle 1-1 Wesentliche Eigenschaften von KIT-Karten 11

Tabelle 2-1 Eigenschaften von autoren- und nutzergesteuerten Methoden ...................................42

Tabelle 3-1 Anteil gefundener Usability-Problemen an gesamten bekannten Usability-Problemen ...60

Tabelle 3-2 Überblick über Hypothesen und Unterhypothesen sowie anzuwendender Methode ......68

Tabelle 4-1 Testgruppen des Usability Tests .................................................................... 76

Tabelle 4-2 Anteil der richtigen Bewertungen zu Frage 5 .........................................................91

Tabelle 4-3 "Richtige" Antworten in Bezug auf die Inhalte der Interpretationshilfe..........................95

Tabelle 4-4 Aufruf von von der Interpretationshilfe nicht präsentierter Inhalte ............................99

Tabelle 4-5 Aufruf von von der Interpretationshilfe nicht angesprochener Bereiche......................100

Tabelle 4-6 Bewertung der Interpretationshilfe durch Multiple-Choice-Auswahl ............................102

Tabelle 4-7 Bewertung der Interpretationshilfe hinsichtlich unterschiedlicher Städte....................108 


\section{ANHANG}

\section{A Quellen zu Kartendaten}

\begin{tabular}{|c|c|c|}
\hline & KOPENHAGEN & WIEN \\
\hline $\begin{array}{l}\text { Standorte } \\
\text { Arztpraxen }\end{array}$ & $\begin{array}{l}\text { Arztsuche Fachärzte } \\
\text { https://notuseborger.scandihealth.net } \\
\text { (aufgerufen Oktober 2014) }\end{array}$ & $\begin{array}{l}\text { Arztsuche Ärztekammer, } 10 \text { häufig nachge- } \\
\text { fragte Fachärzte } \\
\text { http://www.praxisplan.at/ } \\
\text { (aufgerufen Oktober 2014) }\end{array}$ \\
\hline $\begin{array}{l}\text { Preis für } \\
\text { Eigentums- } \\
\text { wohnungen }\end{array}$ & $\begin{array}{l}\text { Durchschnittlicher Verkaufspreis pro } \text { m² }^{2} \\
\text { für verkaufte Eigentumswohnungen die } \\
\text { im Jahr } 2012 \\
\text { http://bolignyheder.boligsiden.dk/2012/1 } \\
\text { 2/kobenhavnske-ejerlejligheder-er-dyrest- } \\
\text { ved-soerne-og-havneindlobet/ } \\
\text { (aufgerufen Oktober 2014) } \\
\text { Durchschnittliche Quadratmeterpreis für } \\
\text { verkaufte Wohnungen, 2013 } \\
\text { http://bolignyheder.boligsiden.dk/2013/0 } \\
\text { 5/her-er-kobenhavns-discountomrader/ } \\
\text { (aufgerufen Oktober 2014) }\end{array}$ & $\begin{array}{l}\text { Preise per Eigentumswohnungen } m^{2} \text { in } € \text {, } \\
\text { November } 2014 \\
\text { www.immopreise.at } \\
\text { (aufgerufen Oktober 2014) }\end{array}$ \\
\hline Einkommen & $\begin{array}{l}\text { Persönliches Einkommen, } 2011 \\
\text { http://politiken.dk/oekonomi/dkoekonom } \\
\text { i/ECE2237017/de-stoerste-danske-byer- } \\
\text { faar-flere-ghettoer-for-rige-og-fattige/ } \\
\text { (aufgerufen Oktober 2014) }\end{array}$ & $\begin{array}{l}\text { Durchschnittliches Nettoeinkommen in Euro } \\
\text { insgesamt: Unselbstständiges Einkommen } \\
\text { nach Bezirken, } 2011 \\
\text { open.wien.at } \\
\text { (aufgerufen Oktober 2014) }\end{array}$ \\
\hline Bevölkerung & $\begin{array}{l}\text { Einwohner pro Zählbezirk, } 2013 \\
\text { http://data.kk.dk/ } \\
\text { (aufgerufen Oktober 2014) }\end{array}$ & $\begin{array}{l}\text { Gesamtbevölkerung pro Zählbezirk, } 2014 \\
\text { http://open.wien.at } \\
\text { (aufgerufen Oktober 2014) }\end{array}$ \\
\hline $\begin{array}{l}\text { Bezirke, } \\
\text { Zählbezirke }\end{array}$ & $\begin{array}{l}\text { http://data.kk.dk/ } \\
\text { (aufgerufen Oktober 2014) }\end{array}$ & $\begin{array}{l}\text { open.wien.at } \\
\text { (aufgerufen Oktober 2014) }\end{array}$ \\
\hline Bebauung & $\begin{array}{l}\text { http://data.kk.dk/ } \\
\text { (aufgerufen Oktober 2014) }\end{array}$ & $\begin{array}{l}\text { https://mapzen.com/metro-extracts } \\
\text { (aufgerufen November 2014) }\end{array}$ \\
\hline Straßen & $\begin{array}{l}\text { https://mapzen.com/metro-extracts } \\
\text { (aufgerufen Dezember 2014) }\end{array}$ & $\begin{array}{l}\text { https://mapzen.com/metro-extracts } \\
\text { (aufgerufen Dezember 2014) }\end{array}$ \\
\hline
\end{tabular}




\title{
B Inhalte der Interpretationshilfe zum Überprüfen der Hypothese
}

\author{
KOPENHAGN
}

Muster Textlicher Inhalt der Interpretationshilfe

Aussagen-

bewertung

\section{Bebauung/Straßen}

1.1. Beginnen wir in Kopenhagen: Ein erster Blick auf die Karte zeigt, dass sich die Standorte der Arztpraxen im Nordosten der Stadt, im Bezirk 'Indre By', konzentrieren.

Beobachtet man die Verteilung der Arztpraxen genauer, er-

1.1.a kennt man, dass sich diese entlang von großen Straßen in die umliegenden Bezirke ausbreiten.

\section{Einkommen}

Im Zusammenhang mit dem Nettoeinkommen der Kopenha-

1.1.c gener, zeigt das grüne Balkendiagramm, dass, bis auf einen Ausreißer, die Anzahl der Arztpraxen in jenen Bezirken konzentriert ist, in denen das Einkommen der Bevölkerung hoch ist.

\section{Kosten für Eigentumswohnung}

Verglichen mit den Quadratmeterpreisen für Eigentumswoh nungen, sammeln sich die Standorte von Ärztlnnen in den dun-

3.1. kelroten Bereichen, wo die Preise hoch sind.

Das Balkendiagramm verdeutlichen diesen Zusammenhang: Mit Ausnahme der teuersten Preisklasse, finden sich mehr Arztpraxen in teureren Gegenden.

\section{Einwohnerdichte}

Im Bezug auf die Einwohnerdichte, finden sich viele Praxen in 1.1.a dunkelblauen Gebieten die eine hohe Einwohnerdichte bedeuten. Allein der Bezirk 'Indre By' stellt eine Ausnahme dar.

Im blauen Balkendiagramm erkennt man, dass abgesehen von der am dichtesten besiedelte Klasse, es mehr Arztpraxen in Gebieten mit hoher Bevölkerungsdichte gibt. 


\section{Bebauung}

1.1.a Betrachten wir nun die Situation in Wien: hier sind beinahe alle bebauten Gebiete der Stadt mit Arztpraxen versorgt.

\section{Straßen}

1.1.c Auch in Wien ist deutlich zu erkennen, dass sich die Arztpraxen entlang der Straßen sternförmig vom geographischen Zentrum der Stadt ausbreiten.

\section{Einkommen}

Im Bezug auf das Einkommensniveau der Wiener Bevölkerung, weisen Bezirke wie 'Innere Stadt', 'Döbling', 'Währing' und 'Hietzing' neben einer hohen Dichte an Arztpraxen (über 30

1.1.a ÄrztInnen pro 10.000 EW) auch ein hohes Nettoeinkommen auf.

Das grüne Balkendiagramm belegt den Eindruck der Karte: Die Anzahl der Arztpraxen ist in Gebieten mit hohem Einkommensniveau höher.

\section{Kosten für Eigentumswohnung}

Weiters zeigt die Karte, dass es viel Standorte von ÄrztInnen in dunkelroten Gebieten gibt, in denen die $\mathrm{m}^{2}$ Preise für Eigen tumswohnung hoch sind.

\section{Stattdessen unwichtiges:}

Die Karte zeigt wie die Kosten für einen $\mathrm{m}^{2}$ Eigentumswohnung in Wien verteilt sind.

Auch in Wien finden sich wenige Ärztlnnen direkt in den teuersten Gebieten. Viele Praxen gibt es jedoch in den zwei darauffolgenden Klassen zwischen 4.000 und $6.000 \mathrm{E} / \mathrm{m}^{2}$ für eine Eigentumswohnung.

Stattdessen unwichtiges:

Aus dem roten Balkendiagramm ist abzulesen, in welcher Preisklasse es wie viele Arztpraxen gibt.

\section{Einwohnerdichte}

In der geographischen Mitte der Stadt finden sich viele Gebiete, die eine hohe Dichte an Einwohnern vorweisen.

Diese dunkelblauen Gebiete sind auch jene, in denen sich auch

Auch im blauen Balkendiagramm wird dieser Zusammenhang deutlich: Vergleicht man die Gebiete mit über 20.000 Einwohner pro $\mathrm{km}^{2}$ mit den Gebieten unter 2.000 Einwohner pro $\mathrm{km}^{2}$ wird dieses besonders auffallend. 


\section{Online Fragebogen zur Auswahl der Testpersonen}

\section{Liebe Teilnehmende!}

Im Rahmen meiner Diplomarbeit an der Technischen Universität Wien führe ich einen User-Test zum Thema Verwendung von Interaktiven Karten durch. Dazu möchte ich Sie gerne als potentiellen Teilnehmenden einladen.

Ist Wien der Ort Ihres Lebensmittelpunkts und sind Sie vertraut im Umgang mit Computern und dem Internet? Dann erfüllen Sie bereits zwei wesentliche Kriterien, um am Test teilnehmen zu können!

Um die weiteren Eignungskriterien zur Teilnahme am User-Test zu prüfen, möchte ich Sie bitten, den folgenden Fragebogen auszufüllen. Die Beantwortung wird nur wenige Minuten beanspruchen. Nachdem Sie den Fragebogen ausgefüllt haben, setzte ich mich mit Ihnen in Verbindung, um bei Eignung den tatsächlichen Test mit Ihnen durchzuführen. Der User-Test wird in den folgenden Wochen auf einem bereitgestellten Computer durchgeführt und dauert etwa 30 Minuten. Der genaue Ort zur Testdurchführung kann nach Absprache entweder in den Räumen der TU Wien oder an anderen vereinbarten Orten in Wien durchgeführt werden. Selbstverständlich werden Ihre Daten vertraulich behandelt. Ihre Angaben dienen ausschließlich wissenschaftlichen Zwecken und werden nicht an Dritte weitergegeben.

Vielen Dank, dass Sie sich für diesen Fragebogen Zeit nehmen. Ich freue mich darauf, viele von Ihnen bei meinem Test begrüßen zu können!

Manuela Stögerer

Beantworten Sie folgende Fragen bitte so korrekt möglich. Bitte achten Sie darauf, den Fragebogen VOLLSTÄNDIG auszufüllen. Nur so kann er für den weiteren Verlauf des Tests verwendet werden.
1. Geschlecht
[Verhältnis 50:50]
$\bigcirc$ Männlich
Weiblich

2. Wohnort

[Wohn- oder Arbeitsort muss Wien sein]

$\bigcirc$ Wien

$\bigcirc$ Anderer

\section{Arbeitsort}

[Wohn- oder Arbeitsort muss Wien sein]

$\bigcirc$ Wien

Andere

4. Beruf

[Ausschluss bei ÄrztInnen]

\section{Alter}




\section{Leiden Sie unter Seheinschränkungen?}

$\bigcirc$ Keine

$\bigcirc$ Kurz- oder Weitsichtig

$\bigcirc$ Farbenblind

[Ausschluss]

$\bigcirc$ Andere:

[Ausschluss bei triftigen]

\section{Verfügen Sie über fließende Deutschkenntnisse?}

$\bigcirc \mathrm{Ja}$

$\bigcirc$ Nein

[Ausschluss]

\section{Welche ist Ihre höchste abgeschlossene Bildung?}

Pflichtschule

[Ausschluss]

$\bigcirc$ Berufsschule/Lehre

[Ausschluss]

$\bigcirc$ Matura (oder gleichwertiges)

$\bigcirc$ Hochschule/Universität

9. In welchem Land haben Sie den Großteil Ihrer Schulausbildung absolviert?

Österreich

$\bigcirc$ andere:

[Ausschluss bei außereuropäischer]

\section{Wie vertraut sind Sie mit der Verwendung von Computer und Internet?}

$\bigcirc$ Ich verwende Computer nur sehr selten und bin daher nicht sehr vertraut mit der Verwendung von Computern und Internet.

[Ausschluss]

$\bigcirc$ Ich verwende das Internet nur für einfache Aufgaben, wie das Abfragen von e-Mails oder einfache Suchabfragen im Netz. Alles was über die Durchführung angelernter Schritte hinaus geht, fällt mir schwer.

[Ausschluss]

$\bigcirc$ Ich verwende das Internet um alltägliche Aufgaben zu bewältigen. Ich weiß, wie und wo ich an Informationen im Netz komme. Hotels oder Flüge zu buchen, sowie Waren zu bestellen, ist kein Problem für mich. [Geeignet]

$\bigcirc$ Ich fühle mich sicher in der Verwendung von Computern und Internet. Ich bin es gewohnt, viele verschiedene Softwareprodukte oder Internetanwendungen für verschiedenste Zwecke zu verwenden.

\section{[Geeignet]}

\section{Welche dieser Aussagen trifft am ehesten auf Sie zu?}

Hinweis: Thematische Karten sind Karten in denen ein spezielles Thema (z.B. Einkommensniveau, Bevölkerungsentwicklung, ..) dargestellt wird. Topografische Karte, die beispielsweise Geländeformen oder andere Gegenstände der Erdoberfläche darstellen, sowie Stadtpläne zählen nicht zu thematischen Karten.

$\bigcirc$ Ich studiere eine Studienrichtung (mind. 6 Semester) mit Kartenbezug, z.B. Kartographie, Raumplanung, Geographie, Geodäsie, Geovisualisierung oder verwandte Studien. [Testgruppe: ExpertInnen]

$\bigcirc$ Ich arbeite/habe gearbeitet in einem Beruf, bei dem ich regelmäßig mit thematischen Karten zu tun habe/hatte. 
In meiner Freizeit beschäftige ich mich mit thematischen Karten oder anderen Datenvisualisierungen.

[Testgruppe: ExpertInnen]

Ich habe weder studienbezogen, beruflich noch durch private Interessen einen Bezug zu thematischen Karten.

[Testgruppe: Neulinge]

Und nun zur letzten Frage:

12. Bitte ordnen Sie den auf der Abbildung dargestellten Städten ihre entsprechenden Namen zu.

Wenn Sie die Städte nicht benennen können, schummeln Sie bitte nicht, da das den Test unbrauchbar macht. Lassen Sie stattdessen die entsprechenden Felder einfach leer.

Stadt A

Stadt B

Stadt C

[Ausschluss bei Erkennen]

Stadt D

Stadt E

[Ausschluss bei nicht Erkennen]
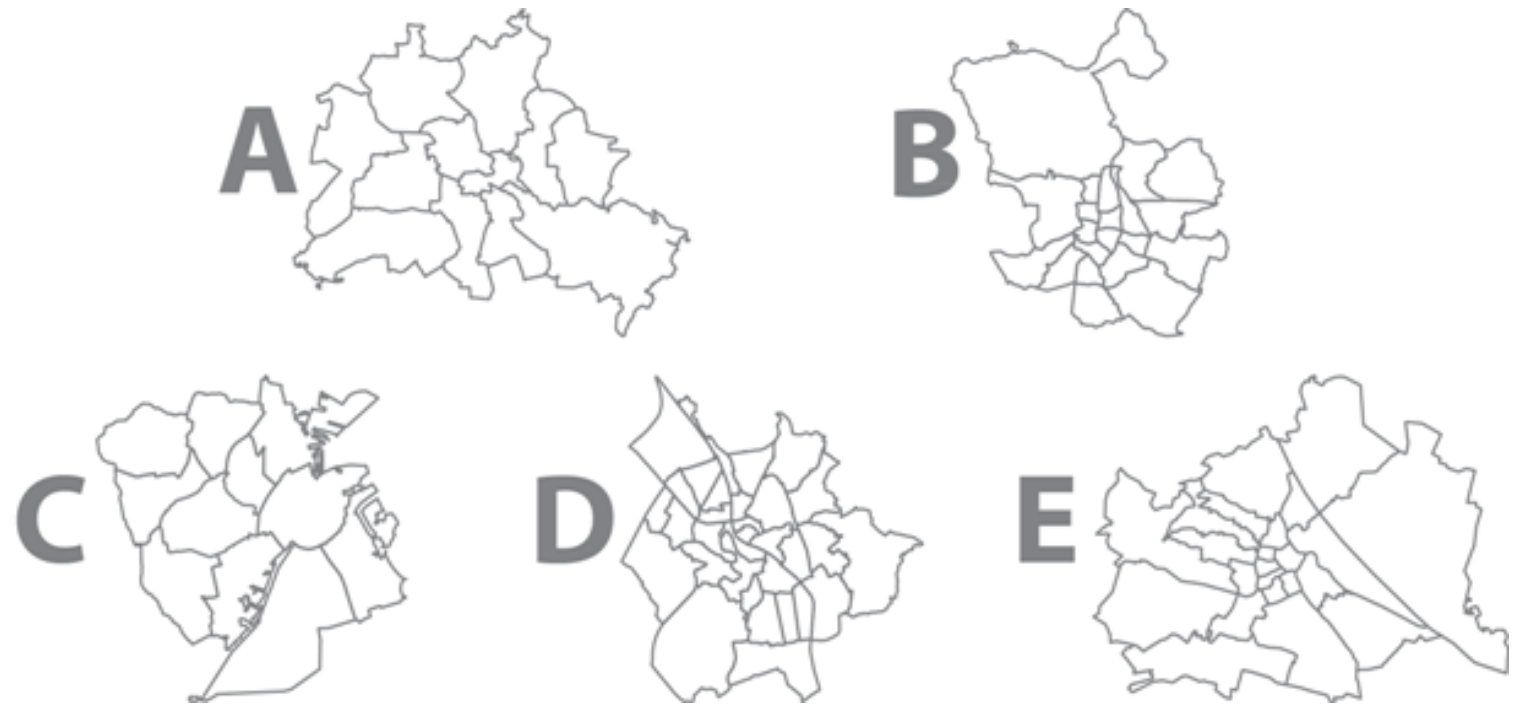

\section{Vielen Dank für Ihre Teilnahme!}

Bitte tragen Sie nun hier Ihre Telefonnummer und/oder e-Mail Adresse ein, damit ich Sie bzgl. des Tests kontaktieren kann.

Name

e-mail Adresse

Telefonnummer

Ihre Antworten wurden gespeichert, Sie können das Browser-Fenster nun schließen. 


\section{Fragebogen des Usability-Test (Vortest, Anwendungstest, Nachtest) für Szenario 1 und Szenario 2}

Szenario 1 (mit richtigen Antworten der Bewertungsaufgabe in Frage5)

\section{ه) Herzlich Willkommen!}

Vielen Dank, dass Sie sich/du dich dazu bereit erklären/erklärst an dieser Studie zum Thema "Verwendung von Interaktiven Karten" teilzunehmen! Selbstverständlich werden Ihre/deine Daten vertraulich behandelt. Ihre/deine Angaben dienen ausschließlich wissenschaftlichen Zwecken und werden nicht an Dritte weitergegeben.

Dieser Test setzt sich verschiedenen Aufgaben zusammen, die ausgeführt werden müssen. Für jede Aufgabe wird einzeln genau erklärt, wie diese zu lösen ist. Die Teilnahme am Test wird in etwa 30 Minuten beanspruchen.

Es geht nicht darum Wissen und Können zu bewerten, sondern darum mehr über die Verwendung von Interaktiven Karten heraus zu finden. Es ist daher wichtig, dass Sie sich/du dich ernsthaft Mühe geben/gibst die Aufgaben zu lösen.

Wie schon besprochen, werden sowohl die Aktionen am Bildschirm alles Gesprochene für diesen Test aufgezeichnet.

(1)) Bevor wir mit dem eigentlichen Test beginnen, möchte ich vorab noch erfahren, ob und welche Erfahrungen mit der Verwendung von interaktiven Karten bestehen.

Am Bildschirm siehst du nun ein Beispiel einer Interaktiven thematischen Karte. Sie zeichnet sich dadurch aus, dass beispielsweise durch ein- und ausblenden oder hinein zoomen die Darstellung der Inhalte verändert werden kann. Zu beachten ist weiters, dass diese Karte sich mit einem speziellem Thema beschäftigt, das sich räumlich darstellen lässt (nämlich wann wo welche Immigranten in Amerika eingetroffen sind). Interaktiven Karten wie Google Maps, die hauptsächlich die Erdoberfläche darstellen, zählen nicht zu den thematischen Karten um die es hier geht.

1. Haben Sie/Hast du die am Bildschirm gezeigte Art von Karten schon einmal verwendet?

noch nie

[Warum]

hin und wieder

[In welchem Zusammenhang haben Sie diese verwendet?]

öfters

[In welchem Zusammenhang haben Sie diese verwendet?]

weiß nicht/kann mich nicht erinnern

[Wenn Antwort 1b,c]

2. Wie empfinden oder bewerten Sie/Wie empfindest oder bewertest du die Verwendung dieser Karten im Allgemeinen?

3. Anhand dieses Schemas: Wie würden Sie/würdest du folgende Punkte zur Benutzerfreundlichkeit dieser interaktiven Karten bewerten? 
Allgemeine Verwendung:

überfordernd

informativ $\square$ weiß nicht

Erfassen von konkreten Informationen:

schwer

leicht

weiß nicht

Zweck:

ansprechend

visualisierte

Kommunikation $\quad \square$ weiß nicht

Spielerei

von Informationen

4) Nun kommen wir zum Hauptteil des Tests. Im diesem geht es darum, ein Beispiel einer interaktive Karte zu verwenden und anschließend einige Fragen zu den darin enthaltenen Inhalten zu beantworten.

Nachdem ich bei dieser Studie von Ihnen/dir lernen möchte, möchte ich dich beim Lösen der Aufgabe nicht beeinflussen. Daher kann ich während der Aufgabenausführung keine Fragen beantworten. Lesen Sie/Lies daher bitte die Aufgabengestellung genau und vollständig durch, bevor Sie/du mit der Aufgabe beginnen/beginnst.

4. In wenigen Augenblicken sehen Sie/siehst du eine interaktive Karte zum Thema "Verteilung von Arztpraxen in Wien und Kopenhagen" am Bildschirm. Diese enthält eine Menge an Informationen enthält, die durch Anklicken und Mausbewegungen ein- und ausgeblendet und somit miteinander in Verbindung gesetzt werden können.

Stellen Sie sich vor Sie müssen/Stell dir vor, du musst aufgrund einer Aufgabe, die Ihnen/dir übertragen wird, herausfinden, in welchen städtischen Gebieten sich viele Arztpraxen konzentrieren. Während der Recherchen finden Sie/findest du diese interaktive Karte, die die Situation in den Städten Wien und Kopenhagen abbildet.

Ziel Ihrer/deiner Aufgabe ist es, die wesentlichen Zusammenhänge zur Verteilung der Arztpraxen in beiden Städten festzustellen. Ihre/Deine Untersuchungen sollen Aussagen nach folgendem Muster ermöglichen:

"In Wien gibt es in Gebieten mit hoher Bevölkerungsdichte viele/wenige Ärzte."

"In Kopenhagen finden sich tendenziell viele Ärzte in Gebieten mit .. "

Es geht also nicht darum konkrete Einzelwerte oder Informationen zu speziellen Bezirken der Karte zu entnehmen, sondern wesentliche Zusammenhänge zu erkennen.

Für Ihre/deine Untersuchungen stehen dir in der Karte jeweils die zwei Reiter "KARTE" und "STATISTIK" für Wien und Kopenhagen zur Verfügung. Verwende diese um die Verteilung aller Facharztpraxen in Kombination mit Einkommensverteilung, $\mathrm{m}^{2}$-Preis für Eigentumswohnungen, Bevölkerungsdichte und den weiteren in der Karte enthaltenen Informationen zu erfassen.

Hinweis: Nachdem Sie/du diese interaktive Karte verwendet haben/hast und diese Aufgabe abgeschlossen ist, bekommen Sie/bekommst du eine Reihe von Aussagen nach dem oben beschriebe- 
nen Muster vorgelegt, die mit richtig oder falsch zu bewerten sind. Damit diese nicht vollständig aus dem Gedächtnis beantwortet werden müssen, können Sie/kannst du unten auf dem Zettel entsprechende Notizen machen.

In der nun folgenden Karte finden Sie/findest du rechts neben der Kartendarstellung weitere Anweisungen, die dich anleiten. Bitte folge diesen über die den "Weiter"-Button. Ihnen stehen/Dir stehen 20 Minuten zur Verfügung, um die Inhalte der Karte ausgiebig zu erkunden. Haben Sie/Hast du vor Ablauf der Zeit das Gefühl, alle wesentlichen Inhalte der Karte erfasst zu haben, kann die Anwendung über den "Anwendung beenden"-Button rechts oben beendet werden.

Um nun mit der aktiven Bearbeitung dieser Aufgabe zu beginnen und die Inhalte der Karte zu studieren, drücken Sie/drücke bitte "Start".

5. Bewerten Sie/Bewerte nun folgende Aussagen anhand Ihrer/deiner Erinnerungen bzw. Notizen. Wissen Sie/Weißt du eine Antwort nicht, raten Sie/rate bitte nicht, sondern wählen Sie/wähle "weiß nicht". bebauten Gebiete der Stadt gleichmäßig verteilt.

5b. In Kopenhagen sammeln sich Arztpraxen entlang von großen Straßen.

5c. In Kopenhagen konzentrieren sich die Arztpraxen in Gebieten in denen das Einkommen der Bevölkerung hoch ist.

5d. In Kopenhagen finden sich tendenziell viele Arztpraxen in Gebieten mit hoher Einwohnerdichte

5e. In Wien sind weite Teile des bebauten Gebiets mit Arztpraxen unterversorgt.

5f. In Wien konzentrieren sich die Arztpraxen entlang von Straßen, die sich sternförmig vom geographischen Zentrum der Stadt ausbreiten.

5g. In Wien ist die Anzahl der Arztpraxen in Gebieten mit hohem Einkommensniveau höher.

5h. In Wien konzentrieren sich Arztpraxen in Gebieten mit höherem Quadratmeterpreis für Eigentumswohnungen.

5i. In Wien gibt es in Gebieten mit hoher Dichte an Einwohnern mehr Arztpraxen als in Gebieten mit geringer Einwohnerdichte. 
4) Nachdem Sie/du Aufgaben gelöst haben/hast:

6. Wie schwer empfanden Sie/empfandest du die eben durchgeführte Bewertung der Aussagen?

leicht

eher leicht

[Warum]

eher schwer

$\square$ schwer

7. Waren die Fragen zu Wien und Kopenhagen gleich schwer zu beantworten?

4) Wie Sie bereits bemerkt haben/du bereits gemerkt hast, gab es neben der Karte auf der rechten Seite textliche Kommentare zu Beschreibung der Karteninhalte.

8. Haben Ihnen/dir die textlichen Kommentare bei der Aufgabenlösung geholfen?

$\begin{array}{lc}\square \text { Ja: } & \text { ( } \square \text { bei beiden Städten } \quad \square \text { vor allem bei Wien } \quad \square \text { vor allem bei Kopenha- } \\ \text { gen) } & \text { [Warum] }\end{array}$

9. Welche der folgenden Aussagen entsprechen am ehesten Ihren/deinen Erfahrungen mit den textlichen Kommentaren in dieser Anwendung?

$\square$ Die textlichen Kommentare helfen die wesentliche Aussage der Karte gezielter wahrzunehmen.

$\square$ In den textlichen Kommentaren wird ohnehin nur das beschrieben, was auf der Karte leicht zu erkennen ist.

Die textlichen Kommentare sind nicht umfassend genug, da noch viel mehr aus den einzelnen Karten herauszulesen ist.

Ohne die textlichen Kommentare hätte ich die Zusammenhänge der Inhalte nicht verstanden.

10. Trifft das eben angekreuzte auf beide Städte gleich zu?

$\square$ für beide Städte gleich $\quad \square$ vor allem auf Wien $\quad \square$ vor allem auf Kopenhagen [Warum] 


\section{[Wenn Antwort 1b,c]}

11. In Frage 3 hast du bereits die Benutzerfreundlichkeit von interaktiven Karten bewertet.

Würden Sie/Würdest du die Bewertung ändern, wären textliche Erklärungen, wie du sie in diesem Kartenbeispiel gesehen hast, generell in allen interaktiven Karten vorhanden?

Allgemeine Verwendung:

überfordernd

informativ $\square$ weiß nicht

Erfassen von konkreten Informationen:

schwer

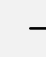

leicht

weiß nicht

Zweck:

ansprechend

Kommunikation

weiß nicht

visualisierte

von Informationen

Spielerei

4) Abschließend möchte ich noch wissen, ob es Schwierigkeiten oder sonst besondere Umstände bei der Bearbeitung der Testaufgabe gab.

12. Haben alle Funktionen der Anwendung funktioniert?

$\square$ ja $\quad \square$ nein [Welche?]

13. Haben Sie/Hast du aus privaten oder beruflichen Gründen Kenntnisse über die dargestellte Thematik?

$\square$ nein $\quad \square$ ja [ in Welchem Zusammenhang?]

14. Haben Sie/Hast du sonst noch generelle Kommentare zur Testanwendung?

nein

[z.B. Hat etwas gestört oder besonders gut gefallen?]

\section{Das war's! Vielen Dank!}


Szenario 2 Antworten der Bewertungsaufgabe in Frage5)

\section{ه) Herzlich Willkommen!}

Vielen Dank, dass Sie sich/du dich dazu bereit erklären/erklärst an dieser Studie zum Thema "Verwendung von Interaktiven Karten" teilzunehmen! Selbstverständlich werden Ihre/deine Daten vertraulich behandelt. Ihre/deine Angaben dienen ausschließlich wissenschaftlichen Zwecken und werden nicht an Dritte weitergegeben.

Dieser Test setzt sich verschiedenen Aufgaben zusammen, die ausgeführt werden müssen. Für jede Aufgabe wird einzeln genau erklärt, wie diese zu lösen ist. Die Teilnahme am Test wird in etwa 30 Minuten beanspruchen.

Es geht nicht darum Wissen und Können zu bewerten, sondern darum mehr über die Verwendung von Interaktiven Karten heraus zu finden. Es ist daher wichtig, dass Sie sich/du dich ernsthaft Mühe geben/gibst die Aufgaben zu lösen.

Wie schon besprochen, werden sowohl die Aktionen am Bildschirm alles Gesprochene für diesen Test aufgezeichnet.

4) Bevor wir mit dem eigentlichen Test beginnen, möchte ich vorab noch erfahren, ob und welche Erfahrungen mit der Verwendung von interaktiven Karten bestehen.

Am Bildschirm siehst du nun ein Beispiel einer Interaktiven thematischen Karte. Sie zeichnet sich dadurch aus, dass beispielsweise durch ein- und ausblenden oder hinein zoomen die Darstellung der Inhalte verändert werden kann. Zu beachten ist weiters, dass diese Karte sich mit einem speziellem Thema beschäftigt, das sich räumlich darstellen lässt (nämlich wann wo welche Immigranten in Amerika eingetroffen sind). Interaktiven Karten wie Google Maps, die hauptsächlich die Erdoberfläche darstellen, zählen nicht zu den thematischen Karten um die es hier geht.

1. Haben Sie/Hast du die am Bildschirm gezeigte Art von Karten schon einmal verwendet?

noch nie

[Warum]

hin und wieder

[In welchem Zusammenhang haben Sie diese verwendet?]

öfters

[In welchem Zusammenhang haben Sie diese verwendet?]

weiß nicht/kann mich nicht erinnern

[Wenn Antwort 1b,c]

2. . Wie empfinden oder bewerten Sie/Wie empfindest oder bewertest du die Verwendung dieser Karten im Allgemeinen?

3. Anhand dieses Schemas: Wie würden Sie/würdest du folgende Punkte zur Benutzerfreundlichkeit dieser interaktiven Karten bewerten? 
Allgemeine Verwendung:

überfordernd

informativ $\square$ weiß nicht

Erfassen von konkreten Informationen:

schwer

leicht

weiß nicht

Zweck:

ansprechend

visualisierte

Kommunikation $\quad \square$ weiß nicht

Spielerei

von Informationen

4) Nun kommen wir zum Hauptteil des Tests. Im diesem geht es darum, ein Beispiel einer interaktive Karte zu verwenden und anschließend einige Fragen zu den darin enthaltenen Inhalten zu beantworten.

Nachdem ich bei dieser Studie von Ihnen/dir lernen möchte, möchte ich dich beim Lösen der Aufgabe nicht beeinflussen. Daher kann ich während der Aufgabenausführung keine Fragen beantworten. Lesen Sie/Lies daher bitte die Aufgabengestellung genau und vollständig durch, bevor Sie/du mit der Aufgabe beginnen/beginnst.

4. In wenigen Augenblicken sehen Sie/siehst du eine interaktive Karte zum Thema "Verteilung von Arztpraxen in Wien und Kopenhagen" am Bildschirm. Diese enthält eine Menge an Informationen, die durch Anklicken und Mausbewegungen ein- und ausblendet und somit miteinander in Verbindung gesetzt werden können.

Stellen Sie sich vor sie müssen/Stell dir vor, du musst aufgrund einer Aufgabe, die Ihnen/dir übertragen wird, herausfinden, in welchen städtischen Gebieten sich viele Arztpraxen konzentrieren. Während der Recherchen finden Sie/findest du diese interaktive Karte, die die Situation in den Städten Wien und Kopenhagen abbildet.

Ziel deiner Aufgabe ist es, die wesentlichen Zusammenhänge zur Verteilung der Arztpraxen in beiden Städten festzustellen. Deine Untersuchungen sollen Aussagen nach folgendem Muster ermöglichen:

"In Wien gibt es in Gebieten mit hoher Bevölkerungsdichte viele/wenige Ärzte."

"In Kopenhagen finden sich tendenziell viele Ärzte in Gebieten mit .."

Es geht also nicht darum konkrete Einzelwerte der Karte zu entnehmen sondern wesentliche Zusammenhänge zu erkennen.

Für deine Untersuchungen stehen Ihnen/dir Karte in der jeweils die zwei Reiter "Karte" und "Statistik" für jeweils Wien und Kopenhagen zur Verfügung. Verwende diese um die Verteilung aller Facharztpraxen in Kombination mit Einkommensverteilung, $\mathrm{m}^{2}$-Preis für Eigentumswohnungen, Bevölkerungsdichte und den weiteren in der Karte enthaltenen Informationen zu erfassen.

Hinweis: Nachdem Sie/du diese interaktive Karte verwendet haben/hast und diese Aufgabe abgeschlossen ist, bekommen Sie/bekommst du eine Reihe von Aussagen nach dem oben beschriebe- 
nen Muster vorgelegt, die mit richtig oder falsch zu bewerten sind. Damit diese nicht vollständig aus dem Gedächtnis beantwortet werden müssen, können Sie/kannst du unten auf dem Zettel entsprechende Notizen machen.

Ihnen /Dir stehen nun 20 Minuten zur Verfügung, um die Inhalte der Karte ausgiebig zu erkunden. Haben Sie/Hast du vor Ablauf der Zeit das Gefühl, alle wesentlichen Inhalte der Karte erfasst zu haben, kann die Anwendung über den "Anwendung beenden"-Button rechts oben beendet werden.

Um nun mit der aktiven Bearbeitung dieser Aufgabe zu beginnen und die Inhalte der Karte zu studieren, drücken Sie/drücke bitte "Start".

5. Bewerten Sie/Bewerte nun folgende Aussagen anhand Ihrer/deiner Erinnerungen bzw. Notizen. Wissen Sie/Weißt du eine Antwort nicht, raten Sie/rate bitte nicht, sondern wählen Sie/wähle "weiß nicht".

5a. In Kopenhagen ist die Verteilung der Arztpraxen bezogen auf die bebauten Gebiete der Stadt gleichmäßig verteilt.

5b. In Kopenhagen sammeln sich Arztpraxen entlang von großen Straßen.

5c. In Kopenhagen konzentrieren sich die Arztpraxen in Gebieten in denen das Einkommen der Bevölkerung hoch ist.

5d. In Kopenhagen finden sich tendenziell viele Arztpraxen in Gebieten mit hoher Einwohnerdichte

5e. In Wien sind weite Teile des bebauten Gebiets mit Arztpraxen unterversorgt.

5f. In Wien konzentrieren sich die Arztpraxen entlang von Straßen, die sich sternförmig vom geographischen Zentrum der Stadt ausbreiten.

5g. In Wien ist die Anzahl der Arztpraxen in Gebieten mit hohem Einkommensniveau höher.

5h. In Wien konzentrieren sich Arztpraxen in Gebieten mit höherem Quadratmeterpreis für Eigentumswohnungen.

5i. In Wien gibt es in Gebieten mit hoher Dichte an Einwohnern mehr Arztpraxen als in Gebieten mit geringer Einwohnerdichte. 
4) Nachdem Sie/du Aufgaben gelöst haben/hast:

6. Wie schwer empfanden Sie/empfandest du die eben durchgeführte Bewertung der Aussagen?

$\square$ leicht

$\square$ eher leicht

$\square$ eher schwer

$\square$ schwer

7. Waren die Fragen zu Wien und Kopenhagen gleich schwer zu beantworten?

$\square$ beide gleich schwer $\quad \square$ Wien leichter $\quad \square$ Wien schwerer

[Warum] $\quad \square$ Kopenhagen leichter $\quad \square$ Kopenhagen schwerer

8. Hätten Sie gerne Unterstützung gehabt zum Erfassen des Karteninhalts gehabt?

$\square$ ja $\square$ nein $\quad$ [Wie hätte diese aussehen können?

4) Abschließend möchte ich noch wissen, ob es Schwierigkeiten oder sonst besondere Umstände bei der Bearbeitung der Testaufgabe gab.

9. Haben alle Funktionen der Anwendung funktioniert?

$\square$ ja $\quad \square$ nein [Welche?]

10. Haben Sie/Hast du aus privaten oder beruflichen Gründen Kenntnisse über die dargestellte Thematik?

$\square$ nein $\quad \square$ ja [ in Welchem Zusammenhang?]

11. Haben Sie/Hast du sonst noch generelle Kommentare zur Testanwendung?

$\square$ nein $\quad$ [z.B. Hat etwas gestört oder besonders gut gefallen?]

\section{Das war's! Vielen Dank!}




\section{ANHANG E Liste der Testpersonen}

$\begin{array}{ll}\text { NEULINGE } \\ \text { TP 73 } & \text { Diplomierte Krankenschwester } \\ \text { TP } 76 & \text { Student Physik } \\ \text { TP } 77 & \text { Studentin Jus, Projektmitarbeiterin } \\ \text { TP } 80 & \text { Elektronik Entwickler } \\ \text { TP } 81 & \text { Business Analystin } \\ \text { TP } 82 & \text { Programmiererin } \\ \text { TP } 83 & \text { Kellner } \\ \text { TP } 84 & \text { Studentin medizinische Informatik } \\ \text { TP } 85 & \text { Promotor } \\ \text { TP } 87 & \text { Student Journalismus \& Medienmanagement }\end{array}$

$\begin{array}{ll}\text { EXPERT_INNEN } \\ \text { TP 66 } & \text { Raumplaner } \\ \text { TP } 67 & \text { Universitätsassistent (Raumplanung) } \\ \text { TP } 68 & \text { Studentin Raumplanung } \\ \text { TP } 69 & \text { Student Geographie } \\ \text { TP } 70 & \text { Immobilien Consultin } \\ \text { TP } 72 & \text { Wissenschaftl. Mitarbeiter: med. Informatik } \\ \text { TP } 75 & \text { Studentin Raumplanung } \\ \text { TP } 78 & \text { Studentin Raumplanung } \\ \text { TP } 88 & \text { Studentin Architektur } \\ \text { TP } 89 & \text { Student Raumplanung, Rettungssanitäter }\end{array}$

ANHANG F Aufzeichnungen des Usability-Tests

Die beigelegte CD beinhaltet Ton- und Bildschirmaufzeichnungen jedes Testteilnehmenden. 